\title{
DIPLOMARBEIT
}

\section{Bewertung der Leistungsfähigkeit von Wärmepumpen im Heiz- und Kühlbetrieb}

\author{
ausgeführt zum Zwecke der Erlangung des akademischen Grades \\ eines Diplom-Ingenieurs \\ unter Leitung von \\ Ao. Univ. Prof. Dipl.-Ing. Dr. techn. Karl Ponweiser
}

E302

Institut für Energietechnik und Thermodynamik

eingereicht an der Technischen Universität Wien,

Fakultät für Maschinenwesen und Betriebswissenschaften

von

Daniel LANGE, BSc

00827275

066445

Wien, im Juli 2018 
Ich habe zur Kenntnis genommen, dass ich zur Drucklegung meiner Arbeit unter der Bezeichnung

\section{Diplomarbeit}

nur mit Bewilligung der Prüfungskommission berechtigt bin.

\section{Eidesstattliche Erklärung}

Ich erkläre an Eides statt, dass die vorliegende Arbeit nach den anerkannten Grundsätzen für wissenschaftliche Abhandlungen von mir selbstständig erstellt wurde. Alle verwendeten Hilfsmittel, insbesondere die zugrunde liegende Literatur, sind in dieser Arbeit genannt und aufgelistet. Die aus den Quellen wörtlich entnommenen Stellen, sind als solche kenntlich gemacht.

Das Thema dieser Arbeit wurde von mir bisher weder im In- noch im Ausland einer Beurteilerin/einem Beurteiler zur Begutachtung in irgendeiner Form als Prüfungsarbeit vorgelegt. Diese Arbeit stimmt mit der von den Begutachterinnen/Begutachtern beurteilten Arbeit überein.

Wien, im Juli 2018 


\section{Kurzfassung}

Der Einsatz von Kaltdampf-Kompressionswärmepumpen zur Bereitstellung von Prozesswärme ist ein aktuell viel diskutiertes Thema, dem mehrere nationale und internationale Forschungsprojekte gewidmet sind und es ist nur eine Frage der Zeit, bis die mit einer Wärmepumpe technisch realisierbare Wärmenutzungstemperatur den betrieblichen Anforderungen mehrerer Industriezweige genügen wird. Die erforderlichen begleitenden Maßnahmen um den wissenschaftlichen Fortschritt auch in die Anwendung transferieren zu können, hinken der allgemeinen Entwicklung jedoch hinterher. So existiert beispielsweise kein zufriedenstellendes Werkzeug, das eine schnelle und vor allem nachvollziehbare Beantwortung der elementaren Fragestellung, was eine Wärmepumpe, eingebettet in die realen technischen und wirtschaftlichen Rahmenbedingungen eines Unternehmens, zu leisten vermag, erlaubt.

Im Rahmen der vorliegenden Arbeit ist der Versuch unternommen worden, zur Schließung dieser Lücke einen Beitrag zu leisten. Aufbauend auf den allgemein bekannten Kennzahlen einer Kompressionswärmepumpe sind größtenteils diagrammbasierte Ansätze zur Ermittlung der wesentlichen technischen und wirtschaftlichen Größen eines Wärmepumpenkreislaufs bis hin zur relativen Energiekosteneinsparung im Heiz- und Kühlbetrieb abgeleitet und, unter Berücksichtigung formelbasierter Zusammenhänge für die absoluten wirtschaftlichen Größen, anhand eines Fallbeispiels dokumentiert und validiert worden.

Die vorliegende Arbeit versteht sich als Diskussionsgrundlage, um die Bewertung der Leistungsfähigkeit eines Wärmepumpenkreislaufs zu vereinheitlichen mit dem Ziel, Hemmnisse auf Seiten der Anwendung abzubauen und im gleichen Zug die Akzeptanz von Wärmepumpen im industriellen Umfeld zu erhöhen. 


\section{Abstract}

The use of mechanical vapor recompression heat pumps to provide process heat is currently a much discussed topic. There are numerous national and international research projects dedicated to this, and it is only a question of time before the required temperature level as made technically possible by a heat pump will be qualified for industrial requirements. However, the necessary accompanying measures to transfer scientific progress into practice are limping behind general development. For instance, there is no satisfactory tool to answer the basic question of what a heat pump, embedded in real-life technological and economic framework condition, can actually do.

In this paper, an attempt is made to help close this gap. Building on the commonly known key technical parameters of the heat pump cycle, this paper uses mostly diagram-based approaches to investigate the relevant technical and economic parameters, culminating in the relative energy conservation in heating and cooling operation, and - by the usage of formula-based approaches for the absolute economic key figures - documents and validates the results with a case study.

This paper aims to be a starting point for discussion, in order to unify the evaluation of the performance of heat pump cycles. This is aimed at breaking down obstacles in the practical implementation, and at the same time increase the acceptance of heat pumps in the industrial sector. 


\section{Inhaltsverzeichnis}

1 Einleitung 1

1.1 Problemstellung . . . . . . . . . . . . . . . . . . 1

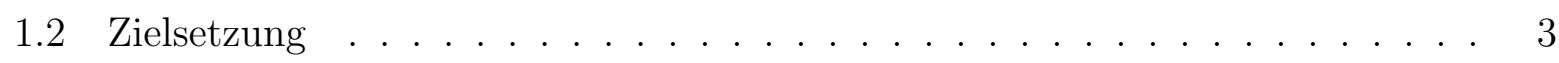

1.3 Stand der Technik . . . . . . . . . . . . . . . . . 4

2 Grundlagen $\quad 6$

2.1 Aufbau einer Wärmepumpe .................... 6

2.2 Idealer Wärmepumpenprozess . . . . . . . . . . . . . . . . 7

2.2.1 Kennzahlen des idealen Wärmepumpenprozesses . . . . . . . . . 8

2.3 Theoretischer Wärmepumpenprozess . . . . . . . . . . . . . . . . 10

2.4 Realer Wärmepumpenprozess . . . . . . . . . . . . . . . . . . . 11

2.4.1 Kennzahlen des realen Wärmepumpenprozesses . . . . . . . . . . 12

2.5 Wärmequelle und Wärmesenke . . . . . . . . . . . . . . . . . 13

3 Bewertung der Leistungsfähigkeit $\quad 14$

3.1 Einführung . . . . . . . . . . . . . . . . . . . . . . . 14

3.2 Annahmen . . . . . . . . . . . . . . . . . . . . 15

3.3 Technische Kennzahlen . . . . . . . . . . . . . . . . . . . . . 20

3.3.1 Maximaler COP im Heizbetrieb . . . . . . . . . . . . . . . . . . 20

3.3.2 Alternativer Ansatz zur Ermittlung von $C O P_{\max }^{\mathrm{H}} \ldots . . . . . . .33$

3.3.3 Realer COP im Heizbetrieb . . . . . . . . . . . . . . . . 43

3.3.4 Realer COP im Heiz- und Kühlbetrieb . . . . . . . . . . . . . . . . 46

3.3.5 Quotient der Nutzwärmeströme . . . . . . . . . . . . . 47

3.3.6 COP einer Kälteanlage . . . . . . . . . . . . . . . . . 49

3.4 Relative wirtschaftliche Kennzahlen . . . . . . . . . . . . . . . . 50

3.4.1 Wirtschaftlichkeitsgrenze im Heizbetrieb . . . . . . . . . . . . 50 
3.4.2 Relative Energiekosteneinsparung im Heizbetrieb . . . . . . . . . . . 61

3.4.3 Relative Kosteneinsparung im Heiz- und Kühlbetrieb . . . . . . . . . 64

3.5 Absolute wirtschaftliche Kennzahlen . . . . . . . . . . . . . . . . 79

3.5.1 Absolute Energiekosteneinsparung . . . . . . . . . . . . . . 79

3.5.2 Investitionskosten . . . . . . . . . . . . . . . . 81

3.5.3 Amortisationsdauer . . . . . . . . . . . . . . . 82

4 Zusammenfassung $\quad 83$

4.1 Technische Kennzahlen . . . . . . . . . . . . . . . . . 83

4.2 Relative wirtschaftliche Kennzahlen . . . . . . . . . . . . . . . 86

4.3 Absolute wirtschaftliche Kennzahlen . . . . . . . . . . . . . . . . 90

5 Anwendung $\quad 92$

5.1 Ausgangssituation . . . . . . . . . . . . . . . . . 92

5.2 Direkte Ermittlung von $\widehat{E}^{\mathrm{H}} \ldots \ldots \ldots \ldots \ldots$. . . . . . . . . . 94

5.3 Inverse Bestimmung von $\vartheta_{\mathrm{S}}^{\max }$ bei gegebenem $\widehat{E}^{\mathrm{H}} \ldots \ldots$. . . . . . . . . 101

5.4 Direkte Ermittlung von $E\left(\Delta t_{\mathrm{P}}\right)$ und $\Delta t_{\mathrm{A}}$ im Heizbetrieb . . . . . . . . . . 104

5.5 Sensitivität der absoluten wirtschaftlichen Kennzahlen . . . . . . . . . . . 109

5.6 Direkte Ermittlung von $E\left(\Delta t_{\mathrm{P}}\right)$ und $\Delta t_{\mathrm{A}}$ im Heiz- und Kühlbetrieb . . . . . 111

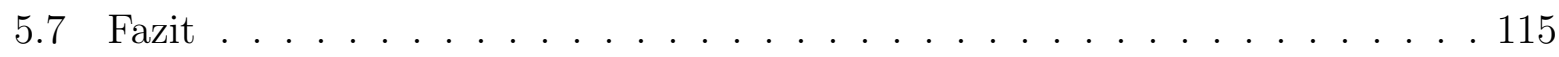

6 Diskussion und Ausblick $\quad 116$

6.1 Technische Kennzahlen . . . . . . . . . . . . . . . . . 116

6.2 Wirtschaftliche Kennzahlen . . . . . . . . . . . . . 118

6.2.1 Relative wirtschaftliche Kennzahlen . . . . . . . . . . . . . 118

6.2.2 Absolute wirtschaftliche Kennzahlen . . . . . . . . . . . . . 120

6.3 Allgemeine Punkte . . . . . . . . . . . . . . . . 120

$\begin{array}{ll}\text { A Diagramme und Tabellen } & 123\end{array}$ 


\section{Abbildungsverzeichnis}

2.1 Aufbau eines Wärmepumpenkreislaufs . . . . . . . . . . . . . . 7

$2.2 T s$-Diagramm eines idealen Wärmepumpenprozesses . . . . . . . . . . . . 8

$2.3 T s$-Diagramm eines theoretischen Wärmepumpenprozesses - Beispiel . . . . . 10

$2.4 T s$-Diagramm eines realen Wärmepumpenprozesses - Beispiel . . . . . . . . . 11

2.5 Schaltbild und Energieströme eines realen Wärmepumpenkreislaufs . . . . . 12

2.6 Temperaturverhältnisse am Wärmepumpenkreislauf . . . . . . . . . . . . 13

3.1 Bestimmung von $T_{\mathrm{Q}}$ und $T_{\mathrm{S}}$ am Wärmepumpenkreislauf $\ldots \ldots \ldots$

3.2 Idealisierter Temperaturverlauf im Kondensator eines theoretischen Wärme-

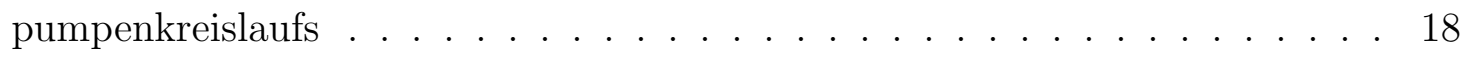

3.3 Idealisierter Temperaturverlauf im Verdampfer eines theoretischen Wärme-

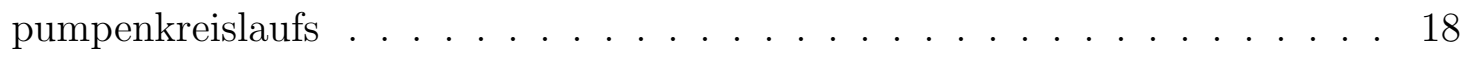

$3.4 \widehat{\Delta T}$ als Funktion von $\Delta T_{\mathrm{SQ}}$ für konstante Werte von $\Delta T_{\mathrm{WT}}$ im Intervall $0{ }^{\circ} \mathrm{C} \leq \Delta T_{\mathrm{SQ}} \leq 25{ }^{\circ} \mathrm{C} \ldots \ldots \ldots \ldots \ldots . \ldots \ldots \ldots$

$3.5 \widehat{\Delta T}$ als Funktion von $\Delta T_{\mathrm{SQ}}$ für konstante Werte von $\Delta T_{\mathrm{WT}}$ im Intervall $25{ }^{\circ} \mathrm{C} \leq \Delta T_{\mathrm{SQ}} \leq 100{ }^{\circ} \mathrm{C} \ldots \ldots \ldots \ldots \ldots . \ldots \ldots$

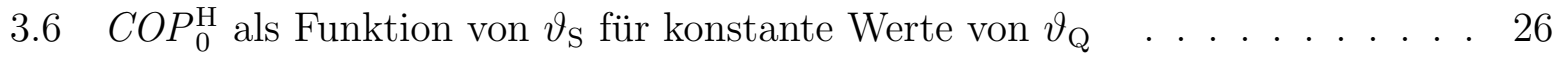

$3.7 C O P_{0}^{\mathrm{H}}$ als Funktion von $\vartheta_{\mathrm{Q}}$ für konstante Werte von $\vartheta_{\mathrm{S}} \quad \ldots \ldots$. . . . . . 26

$3.8 \vartheta_{\mathrm{S}}$ als Funktion von $\vartheta_{\mathrm{Q}}$ im Intervall $0{ }^{\circ} \mathrm{C} \leq \vartheta_{\mathrm{S}} \leq 200{ }^{\circ} \mathrm{C}$ für konstante Werte von $C O P_{0}^{\mathrm{H}} \ldots \ldots \ldots \ldots \ldots \ldots \ldots \ldots \ldots$

$3.9 X_{\mathrm{T}}$ als Funktion von $C O P_{0}^{\mathrm{H}}$ im Intervall $2 \leq C O P_{0}^{\mathrm{H}} \leq 26$ für konstante Werte von $\widehat{\Delta T} \ldots \ldots \ldots \ldots \ldots \ldots \ldots$

$3.10 C O P_{\max }^{\mathrm{H}}$ als Funktion von $C O P_{0}^{\mathrm{H}}$ im Intervall $2 \leq C O P_{0}^{\mathrm{H}} \leq 26$ für konstante Werte von $X_{\mathrm{T}} \ldots \ldots \ldots \ldots \ldots \ldots$ 
$3.11 C O P_{\max }^{\mathrm{H}}$ als Funktion von $C O P_{0}^{\mathrm{H}}$ im Intervall $2 \leq C O P_{0}^{\mathrm{H}} \leq 10$ für konstante

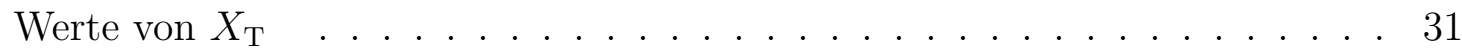

$3.12 C O P_{\max }^{\mathrm{H}}$ als Funktion von $\vartheta_{\mathrm{Q}}$ für konstante Werte von $\vartheta_{\mathrm{S}}$ bei $\Delta T_{\mathrm{WT}}=5{ }^{\circ} \mathrm{C} . \quad 34$

$3.13 C O P_{\max }^{\mathrm{H}}\left(\vartheta_{\mathrm{S}}^{1}, \vartheta_{\mathrm{Q}}, \Delta T_{\mathrm{WT}}^{1}\right)$ und $C O P_{\max }^{\mathrm{H}}\left(\vartheta_{\mathrm{S}}^{2}, \vartheta_{\mathrm{Q}}, \Delta T_{\mathrm{WT}}^{2}\right) \ldots \ldots . \ldots . \ldots . \ldots$

$3.14 \overline{C O P}_{\max }^{\mathrm{H}}$ als Funktion von $\vartheta_{\mathrm{Q}}$ für konstante Werte von $\vartheta_{\mathrm{S}}^{1} \ldots \ldots$. . . . . . 41

$3.15 C O P_{\text {real }}^{\mathrm{H}}$ als Funktion von $C O P_{\max }^{\mathrm{H}}$ für konstante Werte von $\eta_{\mathrm{WP}} \ldots \ldots$. . . . 44

$3.16 C O P_{\text {real }}^{\mathrm{H}}$ als Funktion von $C O P_{\max }^{\mathrm{H}}$ im Intervall $0 \leq C O P_{\max }^{\mathrm{H}} \leq 4$ für konstante Werte von $\eta_{\mathrm{WP}} \ldots \ldots \ldots \ldots$. . . . . . . . . . . 45

$3.17 C O P_{\text {real }}^{\mathrm{H}+\mathrm{K}}$ als Funktion von $C O P_{\text {real }}^{\mathrm{H}} \ldots \ldots \ldots \ldots \ldots$

$3.18 \widehat{Q}$ als Funktion von $C O P_{\text {real }}^{\mathrm{H}} \ldots \ldots \ldots \ldots \ldots$

3.19 Quotient aus $\eta_{\mathrm{B}}$ und $\eta_{\mathrm{A}}$ im Intervall $0,2 \leq \eta_{\mathrm{A}} \leq 1$ für konstante Werte von $\eta_{\mathrm{B}} 58$

3.20 Quotient aus $\eta_{\mathrm{B}}$ und $\eta_{\mathrm{A}}$ im Intervall $0,05 \leq \eta_{\mathrm{A}} \leq 0,2$ für konstante Werte von $\eta_{\mathrm{B}} \ldots \ldots \ldots \ldots \ldots \ldots \ldots \ldots \ldots$

$3.21 F_{\mathrm{B}}^{\mathrm{A}}$ als Funktion von $K_{\mathrm{A}}$ zu $K_{\mathrm{B}}$ im Intervall $0 \leq F_{\mathrm{B}}^{\mathrm{A}} \leq 20$ für konstante Werte von $\eta_{\mathrm{B}} / \eta_{\mathrm{A}} \ldots \ldots \ldots \ldots \ldots \ldots$

$3.22 F_{\mathrm{B}}^{\mathrm{A}}$ als Funktion von $K_{\mathrm{A}}$ zu $K_{\mathrm{B}}$ im Intervall $0 \leq K_{\mathrm{A}} / K_{\mathrm{B}} \leq 2$ und $0 \leq F_{\mathrm{B}}^{\mathrm{A}} \leq 4$ für konstante Werte von $\eta_{\mathrm{B}} / \eta_{\mathrm{A}} \ldots \ldots \ldots \ldots$. . . . . . . . . 60

$3.23 \widehat{E}^{\mathrm{H}}$ als Funktion von $C O P_{\max }^{\mathrm{H}}$ für konstante Werte von $F_{\text {ges }}^{\mathrm{H}} \ldots \ldots . . .63$

$3.24 X_{\mathrm{H}}$ als Funktion von $F_{\text {ges }}^{\mathrm{H}+\mathrm{K}}$ für konstante Werte von $\widehat{Q} \ldots \ldots$. . . . . . . 74

$3.25 X_{\mathrm{H}}$ als Funktion von $F_{\text {ges }}^{\mathrm{H}+\mathrm{K}}$ im Intervall $0 \leq F_{\text {ges }}^{\mathrm{H}+\mathrm{K}} \leq 4$ für konstante Werte von $\widehat{Q} \ldots \ldots \ldots \ldots \ldots \ldots \ldots$. . . . . . . . . . . . . . . . . . . . . . . .

$3.26 X_{\mathrm{K}}$ als Funktion von $F_{\text {ges }}^{\mathrm{H}+\mathrm{K}}$ für konstante Werte von $\widehat{Q} \ldots \ldots . . . . .75$

$3.27 X_{\mathrm{K}}$ als Funktion von $F_{\text {ges }}^{\mathrm{H}+\mathrm{K}}$ im Intervall $0 \leq F_{\text {ges }}^{\mathrm{H}+\mathrm{K}} \leq 4$ für konstante Werte von $\widehat{Q} \ldots \ldots \ldots \ldots \ldots \ldots \ldots$

$3.28 \widehat{E}^{\mathrm{H}+\mathrm{K}}$ als Funktion von $\widehat{E}^{\mathrm{H}}$ für konstante Werte von $X_{\mathrm{H}} \quad \ldots \ldots \ldots$

$3.29 \widehat{E}^{\mathrm{H}+\mathrm{K}}$ als Funktion von $\widehat{E}^{\mathrm{H}}$ für konstante Werte von $X_{\mathrm{K}} \ldots \ldots \ldots$

5.1 Fallbeispiel: Skizze der Quelle . . . . . . . . . . . . . . . . . 93

5.2 Fallbeispiel: Ermittlung von $C O P_{0}^{\mathrm{H}} \ldots \ldots \ldots \ldots$

5.3 Fallbeispiel: Ermittlung von $\widehat{\Delta T} \ldots \ldots \ldots \ldots$. . . . . . . . . . 95

5.4 Fallbeispiel: Ermittlung von $X_{\mathrm{T}} \ldots \ldots \ldots \ldots \ldots$ 
5.5 Fallbeispiel: Ermittlung von $C O P_{\max }^{\mathrm{H}} \ldots \ldots \ldots \ldots$

5.6 Fallbeispiel: Ermittlung von $C O P_{\text {real }}^{\mathrm{H}} \ldots \ldots \ldots \ldots$

5.7 Fallbeispiel: Ermittlung von $\eta_{\mathrm{B}} \mathrm{zu} \eta_{\mathrm{A}} \ldots \ldots \ldots$. . . . . . . . . . . . 99

5.8 Fallbeispiel: Ermittlung von $F_{\text {ges }}^{\mathrm{H}} \ldots \ldots \ldots$. . . . . . . . . . . 99

5.9 Fallbeispiel: Ermittlung von $\widehat{E}^{\mathrm{H}} \ldots \ldots \ldots \ldots$

5.10 Fallbeispiel: Ermittlung des erforderlichen Werts von $C O P_{\max }^{\mathrm{H}}$. . . . . . 102

5.11 Fallbeispiel: Ermittlung von $\overline{C O P_{\max }^{\mathrm{H}}} \ldots \ldots$. . . . . . . . . . . 103

5.12 Fallbeispiel: Ermittlung von $\widehat{Q} \ldots \ldots \ldots 6$

5.13 Fallbeispiel: Ermittlung von $X_{\mathrm{H}} \ldots \ldots \ldots \ldots$. . . . . . . . . . . . . .

5.14 Fallbeispiel: Ermittlung von $\widehat{E}^{\mathrm{H}+\mathrm{K}} \ldots \ldots \ldots \ldots$

\section{Tabellenverzeichnis}

3.1 Ablesehilfe für Abbildung $3.14 \ldots \ldots \ldots$. . . . . . . . . . . . 42

5.1 Fallbeispiel: Betriebliche Randbedingungen . . . . . . . . . . . . . . . 93

5.2 Fallbeispiel: Verwendung von Tabelle $3.1 \ldots \ldots$. . . . . . . . . . . . 103 


\section{Nomenklatur}

\section{Lateinische Symbole}

\begin{tabular}{|c|c|c|}
\hline Zeichen & Einheit & Bedeutung \\
\hline$A$ & ${ }^{\circ} \mathrm{C}$ & Faktor zur Ermittlung von $\overline{C O P}$ \\
\hline$C O P$ & - & Coefficient of Performance \\
\hline$C O P_{\max }$ & - & maximaler $C O P=$ Leistungszahl \\
\hline$C O P_{\text {real }}$ & - & realer $C O P$ \\
\hline$\overline{C O P}$ & - & mittlerer $C O P$ \\
\hline$C O P_{0}$ & - & $C O P$ bei $\Delta T_{\mathrm{WT}}=0{ }^{\circ} \mathrm{C}$ \\
\hline$\widehat{E}$ & - & relative Einsparung \\
\hline$E$ & $€$ o.ä. & absolute Einsparung \\
\hline $\bar{E}$ & $€ / J a h r$ o.ä. & mittlere absolute Einsparung \\
\hline$F_{\mathrm{B}}^{\mathrm{A}}$ & - & Gesamteinflussfaktor - Technologie A und B \\
\hline$F_{\text {ges }}$ & - & Gesamteinflussfaktor \\
\hline$G K$ & $€$ o. ä. & Gesamtkosten \\
\hline$h$ & $\mathrm{~J} / \mathrm{kg}$ & spezifische Enthalpie \\
\hline$I K$ & $€$ o. ä. & Investitionskosten \\
\hline$K$ & $€ / \mathrm{kWh}$ o. ä. & spezifische Kosten Heizen bzw. Kühlen \\
\hline$K^{\mathrm{IK}}$ & $€ /$ kW o.ä. & spezifische Investitionskosten \\
\hline$P$ & - & Kennzeichnung eines Betriebspunkts \\
\hline$p$ & $\mathrm{~N} / \mathrm{m}^{2}$ & absolute Druck \\
\hline$Q$ & $\mathrm{~J}$ & Wärme \\
\hline$\dot{Q}$ & $\mathrm{~W}$ & Wärmestrom \\
\hline$\widehat{Q}$ & - & Quotient der Nutzwärmeströme \\
\hline$s$ & $\mathrm{~J} /(\operatorname{kg} \mathrm{K})$ & spezifische Entropie \\
\hline$T$ & $\mathrm{~K}$ & absolute Temperatur \\
\hline$T_{\mathrm{N}}$ & $\mathrm{K}$ & $\begin{array}{l}\text { hohe Temperatur des idealen Wärme- } \\
\text { pumpenkreislaufs }\end{array}$ \\
\hline$T_{\mathrm{H}}$ & $\mathrm{K}$ & $\begin{array}{l}\text { niedrige Temperatur des idealen Wärme- } \\
\text { pumpenkreislaufs }\end{array}$ \\
\hline$W$ & $\mathrm{~J}$ & Arbeit \\
\hline
\end{tabular}

(wird fortgesetzt) 


\begin{tabular}{lll}
\hline Zeichen & Einheit & Bedeutung \\
\hline$\dot{W}$ & W & Arbeitsstrom \\
$X_{\mathrm{H}}$ & - & Heizkostenfaktor \\
$X_{\mathrm{K}}$ & - & Kühlkostenfaktor \\
$X_{\mathrm{T}}$ & - & Temperaturfaktor
\end{tabular}

\section{Griechische Symbole}

\begin{tabular}{|c|c|c|}
\hline Zeichen & Einheit & Bedeutung \\
\hline$\Delta T$ & ${ }^{\circ} \mathrm{C}$ & Temperaturdifferenz \\
\hline$\Delta T_{\mathrm{WT}}$ & ${ }^{\circ} \mathrm{C}$ & $\begin{array}{l}\text { charakteristische Temperaturdifferenz in den } \\
\text { Wärmetauschern }\end{array}$ \\
\hline$\Delta T_{\mathrm{SQ}}$ & ${ }^{\circ} \mathrm{C}$ & $\begin{array}{l}\text { Temperaturdifferenz zwischen Senke und } \\
\text { Quelle }\end{array}$ \\
\hline$\widehat{\Delta T}$ & - & dimensionslose Temperaturdifferenz \\
\hline$\Delta t$ & Jahr o.ä & Zeitintervall \\
\hline$\Delta t_{\mathrm{P}}$ & Jahr o. ä & Betrachtungszeitraum \\
\hline$\Delta t_{\mathrm{i}}$ & Jahr o. ä & Teilintervall von $\Delta t_{\mathrm{P}}$ \\
\hline$\Delta t_{\mathrm{A}}$ & Jahr o. ä & Amortisationszeitraum \\
\hline$\delta$ & - & relativer Fehler \\
\hline$\varepsilon_{\mathrm{C}}$ & - & Leistungszahl $=$ maximaler COP \\
\hline$\eta$ & - & Wirkungsgrad \\
\hline$\eta_{\mathrm{C}}$ & - & Carnot-Wirkungsgrad \\
\hline$\eta_{\text {kon }}$ & - & $\begin{array}{l}\text { Konversionswirkungsgrad der } \\
\text { stechnologie }\end{array}$ \\
\hline$\eta_{\mathrm{WP}}$ & - & Gütegrad einer Wärmepumpe \\
\hline$\vartheta$ & ${ }^{\circ} \mathrm{C}$ & Temperatur \\
\hline$\vartheta_{1}^{*}$ & - & vorgegebenes Tupel \\
\hline$\vartheta_{2}^{*}$ & - & abgeleitetes Tupel \\
\hline
\end{tabular}


Index - tiefgestellt

\begin{tabular}{cl}
\hline A & Größe der Technologie A \\
B & Größe der Technologie B \\
N & Nutzen \\
Q & Größe der Wärmequelle \\
S & Größe der Wärmesenke \\
WP & Größe der Wärmepumpe \\
el & elektrische Größe \\
kon & Größe der Vergleichstechnologie \\
max & maximaler Wert \\
min & minimaler Wert \\
ij & Größe zwischen den Punkten i und j, z.B. $\dot{Q}_{12}$ \\
& \\
Index - hochgestellt & \\
\hline 1 & Größe des vorgegebenen Tupels \\
2 & Größe des abgeleiteten Tupels \\
i & Größe des idealen Prozesses \\
r & Größe des realen Prozesses \\
$\mathrm{H}$ & Heizen \\
$\mathrm{K}$ & Kühlen \\
$\mathrm{H}+\mathrm{K}$ & Heizen und Kühlen \\
$\mathrm{Kv}$ & Kühlen mit Vergleichstechnologie \\
& \\
\hline &
\end{tabular}




\section{Einleitung}

\subsection{Problemstellung}

Die Herausforderungen, die sich im Zusammenhang mit dem Einsatz von Wärmepumpen ${ }^{1}$ im industriellen Umfeld ergeben, sind vielfältig. Zieht ein Unternehmen die Integration einer Wärmepumpe in Betracht, so steht über allem die Fragestellung der wirtschaftlichen Begründbarkeit eines derartigen Unterfangens. Um diesen zentralen Punkt beantworten zu können, muss auf Basis eines wie auch immer gearteten Berechnungsverfahrens die Leistungsfähigkeit eines gewählten Einbindungskonzepts bestimmt werden. Dass dies zumeist alles andere als trivial ist, zeigt das folgende Beispiel.

Um die wirtschaftliche Sinnhaftigkeit des Einsatzes einer Wärmepumpe zu prüfen, sind zuerst die in Frage kommenden Wärmequellen und Wärmesenken zu erfassen. Im Anschluss gilt es, eine sinnvolle Kombination aus Quelle(n) und Senke(n) zu finden. Die wirtschaftliche Sinnhaftigkeit einer gewählten Kombination ist jedoch auf den ersten Blick nicht ersichtlich, da diese von einer Vielzahl an Aspekten beeinflusst wird. Mögliche Fragestellungen in diesem Zusammenhang sind:

- Passt die Leistung der Quelle, sowohl zeitlich als auch im Bezug auf die absolute Größe, zu der Leistung der Senke?

- Ist das erforderliche Temperaturniveau der Senke mit der ausgewählten Quelle überhaupt erreichbar bzw. welche Auswirkung hat der Temperaturunterschied auf wirtschaftliche Gesichtspunkte?

- Welchen Einfluss hat ein Kühlbedarf der, eventuell auch nur saisonal, monetär zu bewerten ist?

\footnotetext{
${ }^{1}$ Wenn nicht explizit anders angegeben, so handelt es sich hier und im Folgenden um Kompressionswärmepumpen.
} 
Um einen möglichst vollständigen Überblick über etwaige Einsparungspotentiale, die mit dem Einsatz einer Wärmepumpe einher gehen könnten, zu erhalten, sind alle Kombinationen aus Quelle und Senke, unter Berücksichtigung etwaiger zeitlicher Abhängigkeiten, einem rechnerischem Bewertungsverfahren zu unterziehen und im Anschluss zu vergleichen. Hierbei können sowohl frei verfügbare als auch kommerzielle Softwarewerkzeuge eine unterstützende Funktion einnehmen.

Problematisch ist in diesem Zusammenhang die Tatsache, dass das beschriebene Prozedere schon bei einer verhältnismäßig geringen Anzahl an Quellen und Senken sehr zeitintensiv ist. Ein weiterer Nachteil liegt in der Natur der unterstützen Softwarewerkzeuge. Basierend auf der Eingabe (z.B. Temperaturen, Leistungen, spezifische Kosten usw.) werden Kennzahlen errechnet. Mögliche Kennzahlen wären zum Beispiel die relative und absolute Kosteneinsparung, aber auch der Amortisationszeitraum eines gewählten Integrationskonzepts. Je nach Ausprägung der jeweiligen Kennzahl, lässt sich im Anschluss feststellen, ob eine Kombination aus Quelle und Senke, unter Berücksichtigung der getroffenen Annahmen, für die Einbindung einer Wärmepumpe geeignet ist.

Einige wichtige Fragestellungen sind auf diesem Weg jedoch entweder gar nicht, nicht direkt oder nur bei sehr genauer Kenntnis der Materie zu beantworten. Beispiele hierfür sind:

\section{- Die Beantwortung grundsätzlicher Fragen:}

Welche Bedingungen müssen für den wirtschaftlichen Betrieb einer Wärmepumpe erfüllt sein? Welches Kriterium hat an welcher Stelle den Ausschlag gegeben, dass die gewählte Kombination wirtschaftlich nicht umsetzbar ist? Gibt es Kombinationen aus Quelle und Senke die man schon im vom Vorfeld hätte ausschließen können?

\section{- Die Beantwortung inverser Problemstellungen:}

Zum Beispiel, welche Konstellation aus Quelle und Senke ist notwendig um eine relative Energiekosteneinsparung von $15 \%$ zu erreichen? Wie hoch dürfen die spezifischen Investitionskosten einer Wärmepumpe sein, um einen Amortisationszeitraum kleiner als 4 Jahre zu ermöglichen?

\section{- Die Beantwortung diverser sensitiver Fragestellungen:}

Was passiert, wenn die Temperatur der Quelle etwas geringer ausfällt und wie wirkt sich dieser Umstand auf die gesamte Bewertung aus? Wie verhalten sich die wirtschaftlichen Kennzahlen der Wärmepumpe, wenn im nächsten Jahr die spezifischen Kosten für elektrische Energie steigen bzw. sinken? Welchen Einfluss hat ein anderer Gütegrad und welche Auswirkung hat es, wenn die Bewertung mit einem anderen minimalen $\Delta T$ durchgeführt wird? 
Sollte eine Methode bzw. ein Konzept für die einfache, schnelle und nachvollziehbare Beantwortung der oben aufgeführten Fragestellungen gefunden werden, so wären dadurch zwei für die Akzeptanz von Wärmepumpen wesentliche Punkte gewährleistet:

1. Das Verständnis auf der Seite der Anwendung, wie bzw. unter welchen Bedingungen eine Wärmepumpe wirtschaftlich eingesetzt werden kann, würde gestärkt. Weiteres wären positive Auswirkungen auf die Diskussion um die Rolle der Wärmepumpe in Industrie und Gewerbe zu erwarten, da eine einheitliche Diskussionsgrundlage geschaffen wäre.

2. Unternehmen würden dadurch in der Lage versetzt, innerhalb kürzester Zeit herauszufinden, ob und wenn ja, an welcher Stelle es sich lohnt, die Einbindung einer Wärmepumpe anzudenken und könnten mit dieser Information auch direkt weitere Schritte einleiten.

\subsection{Zielsetzung}

Aufbauend auf den in Kapitel 1.1 vorgestellten Herausforderungen, lassen sich die Ziele der vorliegenden Arbeit wie folgt formulieren:

1. Erarbeitung eines diagrammbasierten Konzepts zur Abschätzung der Leistungsfähigkeit einer Wärmepumpe im Heiz- und Kühlbetrieb. Für die Nebenbedingungen gelte:

- Die primäre Kennzahl der Leistungsfähigkeit sei die relative Energiekosteneinsparung. Diese Kennzahl soll auf Grundlage von Diagrammen und einfachen Berechnungen ermittelbar sein. Absolute Kennzahlen können rein auf Formelbasis Verwendung finden.

- Die Ausführungen sollen dem späteren Anwender erlauben zu einer nachvollziehbaren Potentialabschätzung für die wirtschaftliche Integration einer Wärmepumpe zu gelangen und, falls gewünscht, die erzielten Resultate als Grundlage für etwaige nachgelagerte Schritte verwenden zu können.

- Das Konzept soll eine der Aufgabenstellung und dem Grad der für den Einsatz erforderlichen Vorbildung angemessene Komplexität und Bearbeitungsdauer aufweisen.

- Die Anwendung soll, mit Ausnahme eines Taschenrechners, ohne technischen Hilfsmittel und Softwareprodukte möglich sein.

2. Anwendung des Konzepts auf ein repräsentatives Fallbeispiel.

3. Vollständige Dokumentation aller Herleitungen. 
- Hinweise zu den Anforderungen der Dokumentation:

Das vorliegende Dokument versteht sich als Diskussionsgrundlage. Es wird keinesfalls der Anspruch erhoben, dass die eingeführten Kennzahlen den Idealzustand darstellen. Die Dokumentation soll daher so ausgeführt werden, dass es möglich ist, die ggf. als gut befundenen Teile zu übernehmen und für die restlichen Teile andere Ansätze formulieren zu können.

Die hier formulierten Ziele sind ohne Kenntnis der Resultate eventuell etwas abstrakt und geben ein unvollständiges Bild dessen, was eigentlich gesucht ist. Daher sei an dieser Stelle zusätzlich ein Vergleich angeführt:

Die Lebensdauerberechnung eines Wälzlagers kann nach DIN ISO 281 auf ein semigraphisches Verfahren zurückgeführt werden, dass als technisches Hilfsmittel einzig einen Taschenrechner benötigt. Auch wenn die Berechnungsvorschriften einer Norm im Bezug auf die Qualität und die Präzision weit über dem liegen, was im Rahmen der vorliegenden Arbeit als erreichbar anzusehen ist, so können sie dennoch als Beispiel dafür dienen, woran sich gedanklich, im Bezug auf die Zielsetzung dieser Arbeit, orientiert wurde.

\subsection{Stand der Technik}

Es ist festzustellen, dass es für die dieser Arbeit zugrundeliegenden Aufgabenstellungen bisher nur unvollständige Lösungsansätze gibt.

Im Bereich der Auswahl der Quellen und Senken kann die in der Literatur gut dokumentierte Pinchanalyse einen ersten Hinweis geben. Sie ist jedoch in der vorliegenden Arbeit nicht berücksichtigt worden, da sie nur einen kleinen Teilbereich der Gesamtaufgabenstellung abdeckt und vor ihrer Verwendung zu überprüfen wäre, ob die zu erwartenden Resultate der Pinchanalyse im Rahmen der gegebenen Aufgabenstellung in einem günstigen Verhältnis zum einzuplanenden Zeitaufwand stehen. Die mögliche Verwendung der Pinchanalyse wird erneut in Kapitel 6.3 aufgegriffen.

Softwareseitig existieren einige Programme, die in der Lage sind, Teile der Aufgabenstellung zu bearbeiten. Moderne Simulationswerkzeuge, wie zum Beispiel IPSEpro, ASPEN Plus aber auch ENBIPRO, können die mit dem Einsatz einer Wärmepumpe einhergehenden technischen Fragestellungen beantworten. Für eine schnelle Abschätzung der Leistungsfähigkeit ist ihr Einsatz jedoch nicht zu empfehlen, da die von ihnen gebotene Genauigkeit und Aussagekraft der Ergebnisse an dieser Stelle weder gefordert noch monetär und im Bezug auf den zu erwartenden Zeiteinsatz zu rechtfertigen ist. Desweiteren sind diese Programme nicht in der Lage, die elementaren wirtschaftlichen Fragestellungen, die sich im Zusammenhang mit dem Einsatz einer Wärmepumpe ergeben, zu beantworten. 
Die Verwendung nichtkommerzieller Softwarewerkzeuge, wie zum Beispiel EINSTEIN Energy $^{2}$ aber auch das EnPro-Tool ${ }^{3}$, erlaubt die technische und wirtschaftliche Abschätzung des Potentials für die Integration einer Wärmepumpe für eine gewählte Kombination aus Quelle und Senke. Es ist ebenfalls möglich durch Variation der Parameter ein gewisses Gefühl dafür zu bekommen, unter welchen Bedingungen eine Wärmepumpe sinnvoll sein könnte und unter welchen dies nicht der Fall ist. Die Beantwortung der inversen Fragestellung, sprich die Ableitung technischer Parameter bei Vorgabe der wirtschaftlichen Randbedingungen, erlaubt jedoch keines der Softwarewerkzeuge. Als nachteilig erweist sich ebenfalls die Tatsache, dass alle Softwarelösungen nicht ohne Einarbeitungszeit bedienbar und ihre Resultate für den Anwender nicht immer vollständig nachvollziehbar sind. Die Ergebnisse können daher häufig nicht als Grundlage für etwaige nachgelagerte Schritte verwendet werden.

Zusammenfassend lässt sich daher feststellen, dass die vorliegende Arbeit auf verschiedene Grundlagen zurückgreifen kann. Das zu entwickelnde Bewertungskonzept ist in seiner Gesamtheit jedoch als neuartig einzustufen.

\footnotetext{
${ }^{2}$ Verfügbar unter https://www.einstein-energy.net

${ }^{3}$ Verfügbar unter http://wiki.zero-emissions.at/index.php?title=EnPro_Wiki
} 


\section{Grundlagen}

Der prinzipielle Aufbau einer Kompressionswärmepumpe ist allgemein bekannt und bedarf daher keiner literaturgestützten Einführung. Es darf jedoch an dieser Stelle nicht verschwiegen werden, dass sich dieses Kapitel strukturell an den Ausführungen in "Die Wärmepumpe in der Verfahrenstechnik" [4] orientiert. Wie bereits in Kapitel 1 erwähnt, werden die Wörter Wärmepumpe und Kompressionswärmepumpe in der vorliegenden Arbeit gleichbedeutend verwendet.

\subsection{Aufbau einer Wärmepumpe}

Eine Wärmepumpe besteht aus den Komponenten:

- Verdampfer

- Verdichter

- Kondensator

- Drossel

welche wie in Abbildung 2.1 verschaltet einen Wärmepumpenkreislaufs bilden. Das Arbeitsmedium des Wärmepumpenkreislaufs vollzieht im Sinne eines linksläufigen Kreisprozesses mehrere Zustandsänderungen. Gedanklich können die Eckpunkte dieser Zustandsänderungen aufgeilt werden in Zustände bei hohem Druck und hoher Temperatur (Punkte 3 und 4) sowie Zustände bei niedrigem Druck und niedriger Temperatur (Punkte 1 und 2). Ausgehend vom Zustand nach der Drossel wird das Arbeitsmedium zuerst vollständig verdampft und im Anschluss mithilfe des Verdichters auf ein höheres Druck- und Temperaturniveau gebracht, bei welchem es in weiterer Folge im Kondensator vollständig kondensiert wird. Nach der Kondensation erfolgt die Drosselung auf das Druckniveau des Verdampfers.

Unabhängig davon, welcher Ansatz zur Einführung bzw. zur Definition des Grundprinzips der Wärmpumpe Verwendung findet, lässt sich zusammenfassend feststellen, dass eine Wärmepumpe einen Wärmestrom bei niedriger Temperatur aufnimmt und mittels Energiezufuhr einen Wärmestrom bei höherer Temperatur abgibt [4]. 


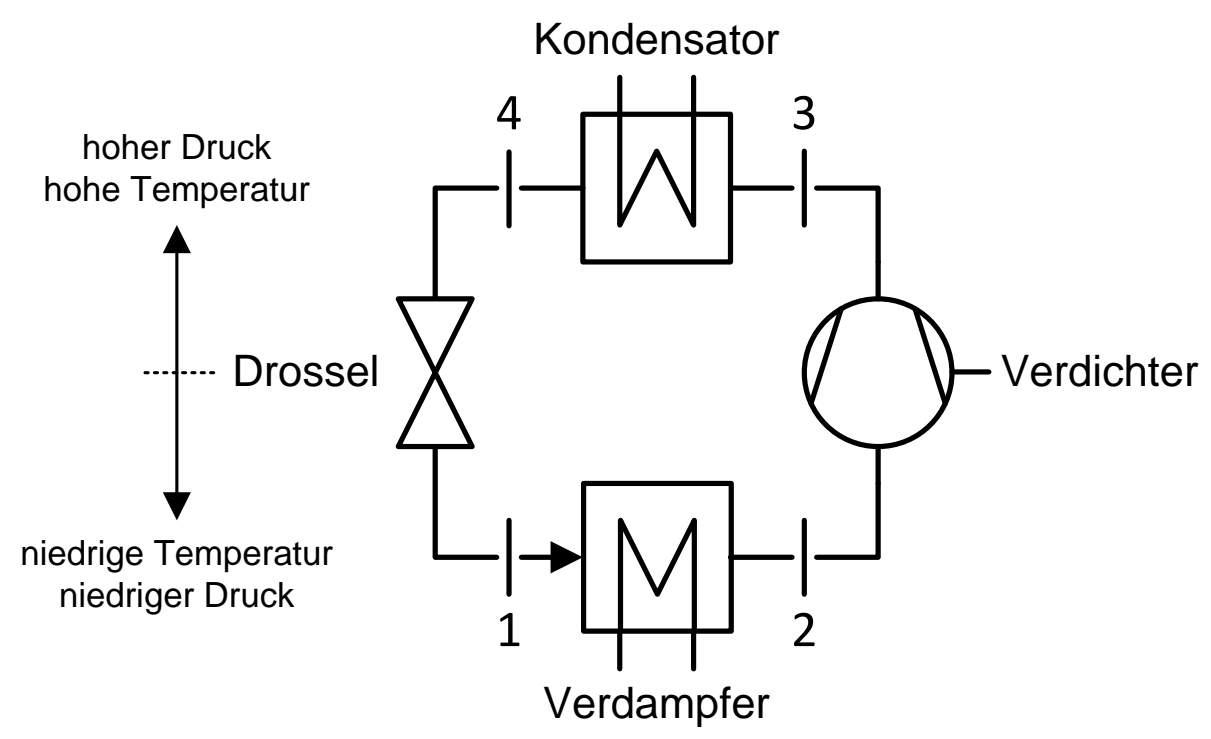

Abbildung 2.1: Aufbau eines Wärmepumpenkreislaufs

Zur thermodynamischen Beschreibung des Wärmepumpeprozesses bietet es sich an, die in [4] eingeführte Aufteilung in einen idealen, einen theoretischen und einen realen Prozess beizubehalten. Diese Aufteilung ist, mit Vorgriff auf Kapitel 3.3, besonders hervorzuheben, da ein über Kennzahlen ausdrückbarer Zusammenhang zwischen dem idealen und dem realen Prozess existiert, welcher die Grundlage dieser Arbeit darstellt.

\subsection{Idealer Wärmepumpenprozess}

Ein idealer Wärmepumpenprozess lässt sich durch einen linksläufigen Carnot-Prozess darstellen. Im Allgemeinen besteht ein linksläufiger Carnot-Prozess aus der Hintereinanderschaltung der folgenden, in Strömungsrichtung des Arbeitsmediums angeschriebenen, idealisierten Teilprozesse [3]:

- Teilprozess 1: isentrope Verdichtung

- Teilprozess 2: isotherme reversible Abgabe von Wärme

- Teilprozess 3: isentrope Expansion

- Teilprozess 4: isotherme reversible Aufnahme von Wärme

Abbildung 2.2 zeigt einen idealen Wärmepumpenprozess unter Verwendung der in Abbildung 2.1 eingeführten Punkte. Der hochgestellte Index i kennzeichnet die über die Systemgrenze geführten Größen des idealen Vergleichsprozesses. 
In Analogie zur Feststellung, dass eine Wärmepumpe einen Wärmestrom bei niedriger Temperatur aufnimmt und mittels Energiezufuhr einen Wärmestrom bei höherer Temperatur abgibt, beschreibt die Temperatur $T_{\mathrm{H}}$ in Abbildung 2.2 die hohe Temperatur bei der isothermen reversiblen Abgabe von Wärme und die Temperatur $T_{\mathrm{N}}$ die niedrige Temperatur bei der isothermen reversiblen Aufnahme von Wärme. Die Temperaturen $T_{\mathrm{H}}$ und $T_{\mathrm{N}}$ sind Temperaturen auf der absoluten Temperaturskala und besitzen die Einheit Kelvin.

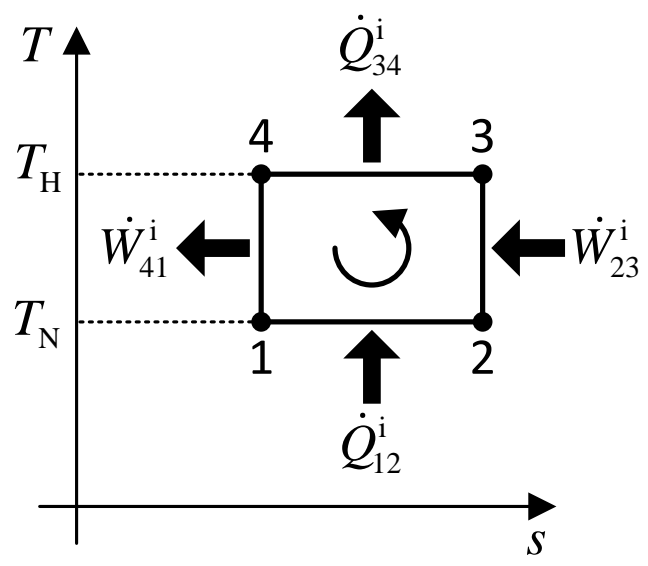

Abbildung 2.2: Ts-Diagramm eines idealen Wärmepumpenprozesses

\subsubsection{Kennzahlen des idealen Wärmepumpenprozesses}

Zur Bestimmung der Effizienz einer Wärmpumpe hat sich die Leistungszahl $\varepsilon$, welche im Englischen auch COP (Coefficient of Performance) genannt wird, etabliert. Der maximale COP eines Wärmepumpenprozesses bzw. die auf den Carnot-Prozess bezogene Leistungszahl, ist definiert als der Quotient aus dem im idealen Vergleichsprozess abgeführten Wärmestrom $\dot{Q}_{\mathrm{ab}}^{\mathrm{i}}$ und dem im idealen Vergleichsprozess Netto zugeführten Arbeitsstrom $\dot{W}^{\mathrm{i}}$ :

$$
C O P_{\max }=\frac{\dot{Q}_{\mathrm{ab}}^{\mathrm{i}}}{\dot{W}^{\mathrm{i}}}=\varepsilon_{\mathrm{C}}
$$

Anmerkung: Der 1. Hauptsatz der Thermodynamik besagt, dass über die Systemgrenze zugeführte Energie die innere Energie des Systems erhöht. Dadurch sind Wärmeströme und Ströme an Arbeit, die das System verlassen, aus streng thermodynamischer Sicht stets kleiner Null. Um nicht mit negativen Energieströmen arbeiten zu müssen, werden Energieströme in jene Richtung eingezeichnet, in welcher sie fließen, jedoch für die Ableitung von Kennzahlen jeweils positiv definiert. Daher gilt zum Beispiel für den Wärmestrom aus Gleichung (2.1):

$$
\dot{Q}_{\mathrm{ab}}^{\mathrm{i}}=-\dot{Q}_{34}^{\mathrm{i}}
$$


Es ist möglich und in weiterer Folge notwendig, das Ergebnis aus Gleichung (2.1) in einen funktionalen Zusammenhang zu überführen, der alleinig von den Temperaturen $T_{\mathrm{H}}$ und $T_{\mathrm{N}}$ abhängt. Um die Größen aus Abbildung 2.2 für Gleichung (2.1) verwenden zu können, wird gesetzt:

$$
\dot{Q}_{\mathrm{zu}}^{\mathrm{i}}=\dot{Q}_{12}^{\mathrm{i}} \quad \dot{Q}_{\mathrm{ab}}^{\mathrm{i}}=-\dot{Q}_{34}^{\mathrm{i}}
$$

Die Energieübertragung in Form von Wärme zwischen den Punkten 1 und 2 sowie 3 und 4 erfolgt isotherm und reversibel. Die Zustandsänderung zwischen den Punkten 2 und 3 beziehungsweise 4 und 1 verläuft isentrop. Daraus folgt für die Entropiebilanz des Systems:

$$
\frac{\dot{Q}_{\mathrm{zu}}^{\mathrm{i}}}{T_{\mathrm{N}}}=\frac{\dot{Q}_{\mathrm{ab}}^{\mathrm{i}}}{T_{\mathrm{H}}}
$$

Für den idealen Wärmepumpenprozess in Abbildung 2.2 gilt, dass die Fläche 1234 dem Netto zugeführten Arbeitsstrom $\dot{W}^{\mathrm{i}}$ entspricht.

Aus dem 1. Hauptsatz der Thermodynamik folgt:

$$
\dot{W}^{\mathrm{i}}=\dot{Q}_{\mathrm{ab}}^{\mathrm{i}}-\dot{Q}_{\mathrm{zu}}^{\mathrm{i}}
$$

Unter Verwendung von Gleichung (2.4) folgt für Gleichung (2.1):

$$
C O P_{\max }=\frac{\dot{Q}_{\mathrm{ab}}^{\mathrm{i}}}{\dot{W}^{\mathrm{i}}}=\frac{\dot{Q}_{\mathrm{ab}}^{\mathrm{i}}}{\dot{Q}_{\mathrm{ab}}^{\mathrm{i}}-\dot{Q}_{\mathrm{zu}}^{\mathrm{i}}}
$$

Das umgeformte Resultat der Entropiebilanz:

$$
\dot{Q}_{\mathrm{zu}}^{\mathrm{i}}=\dot{Q}_{\mathrm{ab}}^{\mathrm{i}} \frac{T_{\mathrm{N}}}{T_{\mathrm{H}}}
$$

ermöglicht die Elimination der Wärmeströme in der Bestimmungsgleichung des maximalen COPs:

$$
\begin{aligned}
C O P_{\max } & =\frac{\dot{Q}_{\mathrm{ab}}^{\mathrm{i}}}{\dot{Q}_{\mathrm{ab}}^{\mathrm{i}}-\dot{Q}_{\mathrm{zu}}^{\mathrm{i}}}=\frac{\dot{Q}_{\mathrm{ab}}^{\mathrm{i}}}{\dot{Q}_{\mathrm{ab}}^{\mathrm{i}}-\dot{Q}_{\mathrm{ab}}^{\mathrm{i}} \frac{T_{\mathrm{N}}}{T_{\mathrm{H}}}}=\frac{1}{1-\frac{T_{\mathrm{N}}}{T_{\mathrm{H}}}} \\
\longrightarrow C O P_{\max } & =\frac{T_{\mathrm{H}}}{T_{\mathrm{H}}-T_{\mathrm{N}}}
\end{aligned}
$$

mit der Forderung:

$$
C O P_{\max }>1
$$




\subsection{Theoretischer Wärmepumpenprozess}

Der theoretische Wärmepumpenprozess ist, im Gegensatz zum idealen Wärmepumpenprozess, kein standardisierter Prozess. Nach [4] setzt sich der theoretische Wärmepumpenprozess aus den folgenden, für die Punkte nach Abbildung 2.1 angeschriebenen, Zustandsänderungen des Arbeitsmediums zusammen:

- $1 \rightarrow$ 2: isobare Wärmeaufnahme bis zur vollständigen Verdampfung

- $2 \rightarrow 3$ : isentrope Verdichtung auf das Druckniveau des Kondensators

- $3 \rightarrow$ 4: isobare Wärmeabgabe bis zur vollständigen Kondensation

- $4 \rightarrow$ 1: isenthalpe Drosselung auf das Druckniveau des Verdampfers

Abbildung 2.3 zeigt das Ts-Diagramm für einen theoretischen Wärmepumpenprozess mit dem Arbeitsmedium R134a, einem Verdampferdruck von 7 bar und einem Kondensatordruck von 32 bar.

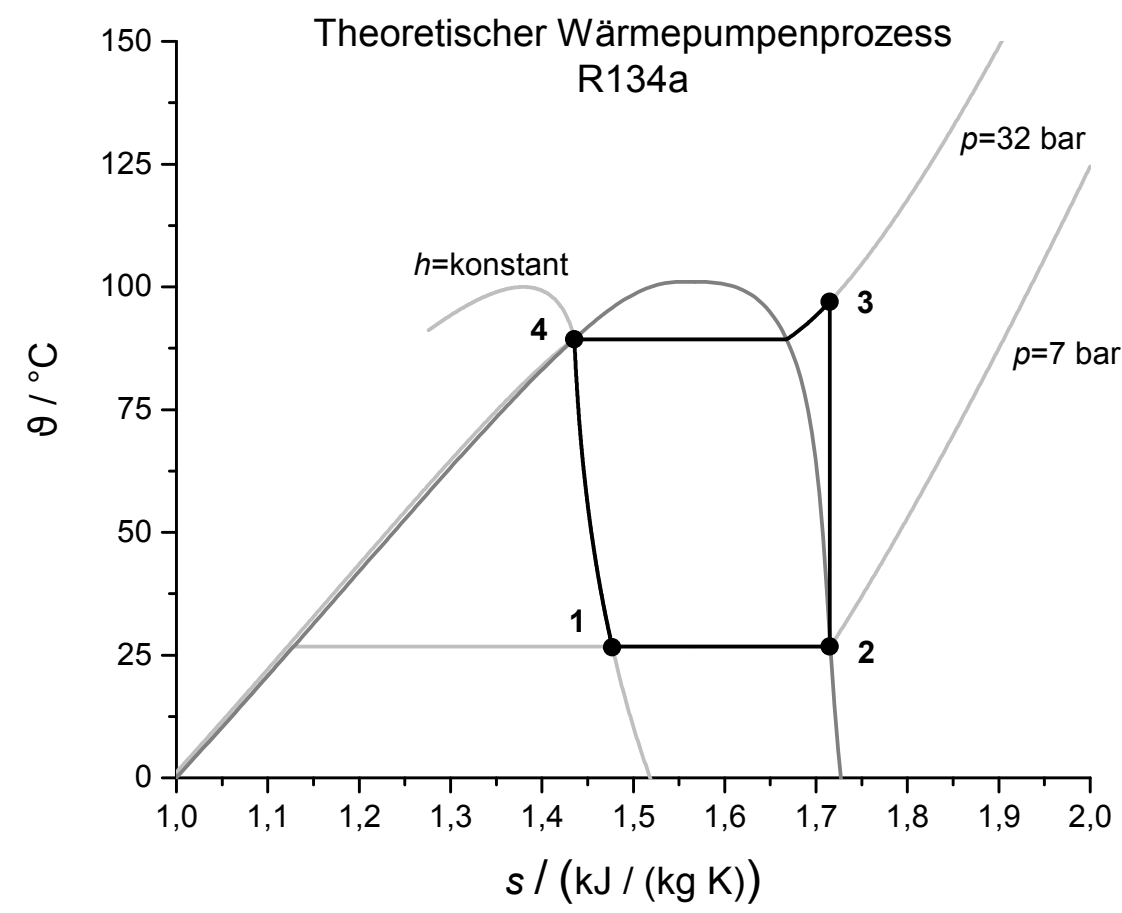

Abbildung 2.3: Ts-Diagramm eines theoretischen Wärmepumpenprozesses - Beispiel

Es ist grundsätzlich möglich, dem theoretischen Wärmepumpenprozess eigene Kennzahlen und damit auch ein eigenes Formelwerk zuzuordnen. In der vorliegenden Arbeit dient der theoretische Wärmepumpenprozess jedoch primär der Verbesserung der Nachvollziehbarkeit des Abstraktionswegs vom idealen über den theoretischen zum realen Wärmepumpenprozess. Auf eine Ausführung der Berechnungsvorschriften des theoretischen Wärmepumpenprozesses ist daher an dieser Stelle verzichtet worden. 


\subsection{Realer Wärmepumpenprozess}

Der reale Wärmepumpenprozess weicht an mehreren Stellen von den Annahmen des theoretischen Wärmepumpenprozesses ab. Zu diesen Abweichungen, angeschrieben für die Punkte nach Abbildung 2.1, zählen unter anderem:

- $1 \rightarrow 2$ : Die Wärmeaufnahme erfolgt nicht isobar sondern mit Druckverlust behaftet.

- $2 \rightarrow 3$ : Die Verdichtung erfolgt nicht isentrop sondern mit Entropiezunahme.

- $3 \rightarrow$ 4: Die Wärmeabgabe erfolgt nicht isobar sondern mit Druckverlust behaftet.

- $4 \rightarrow$ 1: Die Drosselung erfolgt nicht isenthalp sondern mit Enthalpieverringerung.

Abbildung 2.4 zeigt exemplarisch, wie das Ts-Diagramm eines realen Wärmepumpenprozesses aussehen könnte. Zur besseren Vergleichbarkeit entsprechen Arbeitsmedium und Druckparameter den Werten des theoretischen Wärmepumpenprozesses aus Abbildung 2.3.

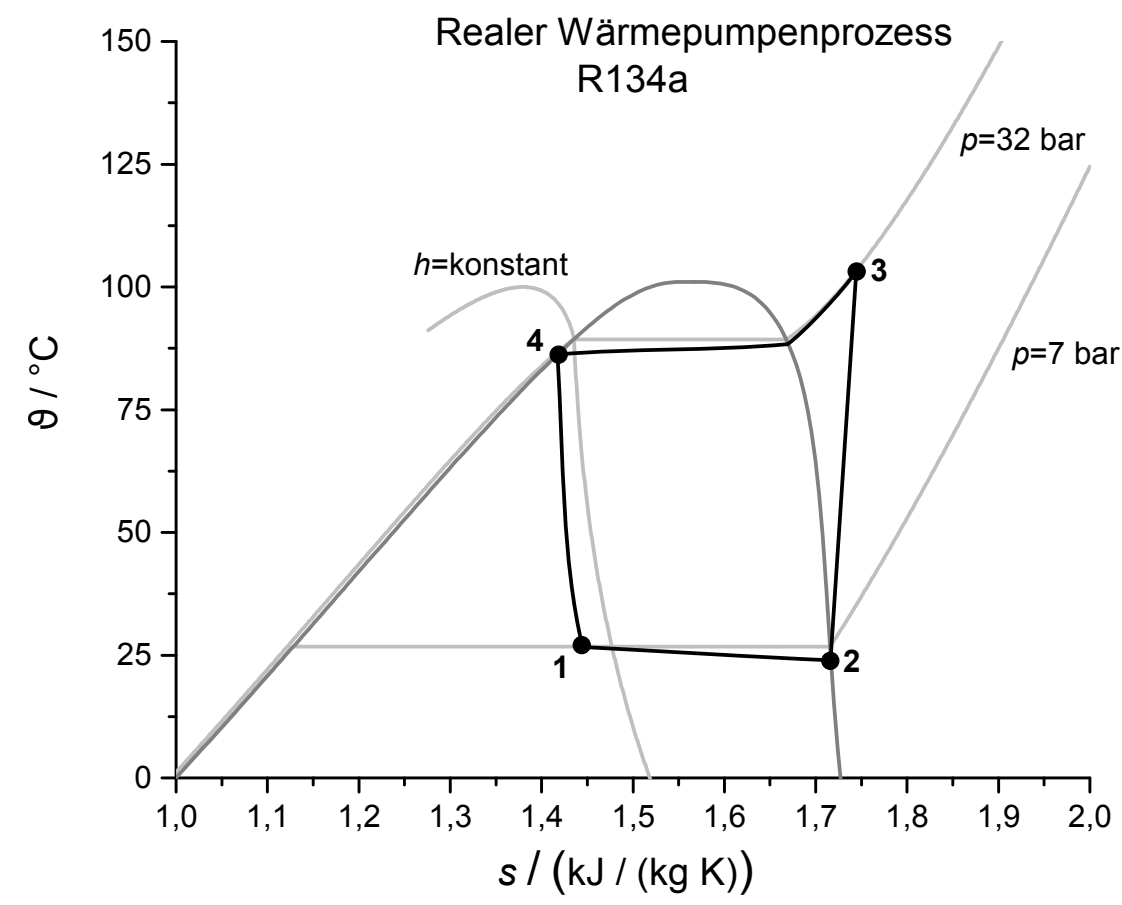

Abbildung 2.4: Ts-Diagramm eines realen Wärmepumpenprozesses - Beispiel

Eine detaillierte Aufstellung und Diskussion der Unterschiede zwischen dem realen und dem theoretischen Wärmepumpenprozess bietet [4]. Für die vorliegende Arbeit genügt die Feststellung, dass die Annahmen, die für die Zustandsänderungen des Arbeitsmediums des theoretischen Wärmepumpenprozesses getätigt wurden, nicht auf den realen Wärmepumpenprozess übertragbar sind. Daraus resultiert, dass die Eckpunkte des realen Wärmepumpenprozesses auch nicht ohne weiteres berechenbar sind. 


\subsubsection{Kennzahlen des realen Wärmepumpenprozesses}

Abbildung 2.5 zeigt die an einem realen Wärmepumpenprozess beteiligten, durch den hochgestellten Index r gekennzeichneten, Energieströme:

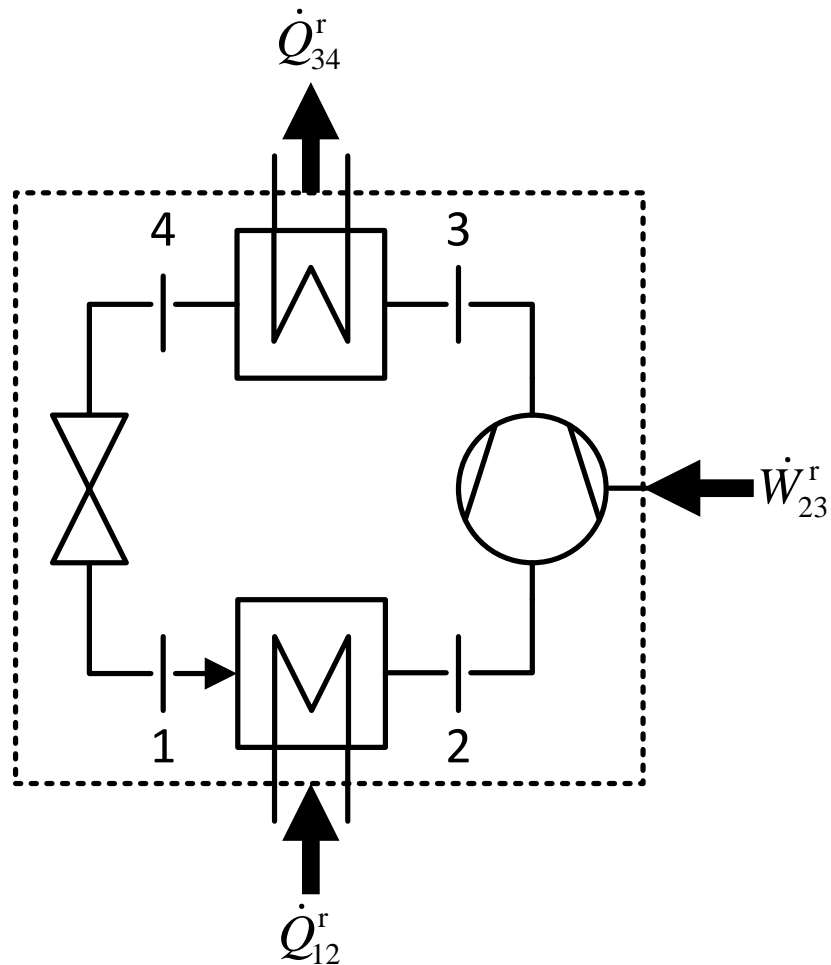

Abbildung 2.5: Schaltbild und Energieströme eines realen Wärmepumpenkreislaufs

Für die Berechnung des maximalen COPs wird der real zwischen den Punkten 3 und 4 abgegebene Wärmestrom mit dem real zugeführten Arbeitsstrom normiert:

$$
C O P_{\text {real }}=\frac{\dot{Q}_{34}^{\mathrm{r}}}{\dot{W}_{23}^{\mathrm{r}}}
$$

Eine weitere Kennzahl, die einen Zusammenhang zwischen dem realen und dem theoretischen Wärmepumpenprozess herstellt, ist der Quotient aus dem realen und dem maximalen COP, welcher auch Gütegrad genannt wird:

$$
\eta_{\mathrm{WP}}=\frac{C O P_{\text {real }}}{C O P_{\text {max }}}
$$




\subsection{Wärmequelle und Wärmesenke}

Als Wärmequelle wird jenes Medium bezeichnet, das den Verdampfer des Wärmepumpenkreislaufs durchströmt und sich dabei abgekühlt. Der im Rahmen der Abkühlung der Wärmequelle an das Arbeitsmedium des Wärmenpumpenkreislaufs übertragene Wärmestrom, ist jener Wärmestrom, den die Wärmepumpe laut Definition (vgl. Kapitel 2.1) bei niedriger Temperatur aufnimmt. In Analogie dazu, ist die Wärmesenke definiert als das Medium, das sich beim Durchströmen des Kondensators durch Aufnahme des Wärmestroms, den die Wärmepumpe bei hoher Temperatur abgibt, erwärmt.

Daraus folgt, dass das Arbeitsmedium der Wärmepumpe, die Wärmequelle und die Wärmesenke temperaturtechnisch gesehen umschließt. Für jeden Zeitpunkt und für jeden Ort (bei gedanklicher Darstellung der Temperatur der am Wärmeaustausch beteiligten Medien über der Länge der Wärmetauscher) müssen daher die in Abbildung 2.6 exemplarisch gezeigten Temperaturverhältnisse eingehalten werden.

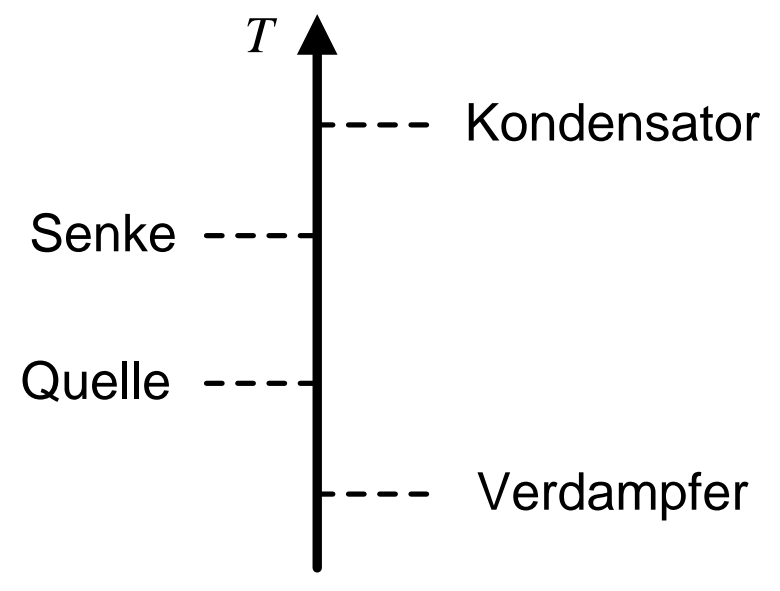

Abbildung 2.6: Temperaturverhältnisse am Wärmepumpenkreislauf

Anmerkung: Die Ausdrücke Wärmequelle und Quelle werden im Folgenden synonym verwendet. Selbiges gilt für die Ausdrücke Wärmesenke und Senke. 


\section{Bewertung der Leistungsfähigkeit}

\subsection{Einführung}

Die Ausführungen in den Kapiteln 2.2 bis 2.4 beschreiben den Wärmepumpenprozess, was in diesen Kapiteln gleichbedeutend ist, mit den Zustandsänderungen des Arbeitsmediums des Wärmepumpenkreislaufs. Für die weiteren Ausführungen sind in diesem Zusammenhang zwei Punkte hervorzuheben:

1. Die Berechnung der realen Zustände des Arbeitsmediums des Wärmepumpenkreislaufs ist ohne Berücksichtigung der realen Geometrie der Wärmetauscher, des Teillastverhaltens und der Stoffdaten nicht bzw. nur sehr eingeschränkt möglich.

2. Aus Sicht des Anwenders dominiert die Fragestellung, welche Zustände sich auf der Seite der Wärmequelle und der Wärmesenke einstellen.

Auf Basis dieser Erkenntnisse wird in diesem Kapitel versucht, möglichst allgemeine Zusammenhänge abzuleiten, die zur Berücksichtigung des realen Verhaltens eines Wärmepumpenkreislaufs nicht mehr als den Gütegrad benötigen, im Bezug auf ihren Formelapparat nicht maßgeblich über die in Kapitel 2 vorgestellten Berechnungsvorschriften hinausgehen, leicht $\mathrm{zu}$ visualisieren sind und es ermöglichen, die wesentlichen nutzerseitigen Fragestellungen bei der Bewertung der Leistungsfähigkeit einer Wärmepumpe zu beantworten. Das Wort Leistungsfähigkeit beschreibt in diesem Zusammenhang, wie ausgeprägt das wirtschaftliche Potential für die Integration einer Wärmepumpe ist. Die folgenden Begriffe bzw. Größen können daher als unmittelbar mit der Leistungsfähigkeit verbunden angesehen werden:

- Die technische und wirtschaftliche Grenze, bei deren Unterschreitung der Einsatz einer Wärmepumpe wirtschaftlich keinen Sinn ergibt.

- Die relative und die absolute Kosteneinsparung, untergliedert nach der Art des Betriebs der Wärmepumpe.

- Die Investitionskosten sowie die Amortisationsdauer. 


\subsection{Annahmen}

\section{Temperaturunterschied in den Wärmetauschern}

In der Gleichung des maximalen COPs (vgl. Gleichung (2.7)) kommen nur die Temperaturen des Arbeitsmediums der Wärmepumpe vor. Für die Anwendung sind jedoch die Temperaturen auf der Seite der Senke bzw. der Quelle von Relevanz. Der Temperaturunterschied in den Wärmetauschern, zwischen dem wärmeaufnehmenden Medium und dem wärmeabgebenden Medium, ist jedoch in den wenigsten Fällen bekannt (vgl. Abbildung 2.4).

Im Rahmen dieser Arbeit wird daher die folgende Annahme für den Temperaturunterschied in den Wärmetauschern getroffen:

- Die für den Wärmeübergang in den Wärmetauschern notwendige Temperaturdifferenz zwischen den am Wärmeaustausch beteiligten Strömen, wird sowohl quellen- als auch senkenseitig über einen konstanten charakteristischen Wert $\Delta T_{\mathrm{WT}}$ berücksichtigt.

\section{Temperatur der Quelle und der Senke}

Die Art und Weise, wie die Temperatur der Quelle bzw. der Senke in die Bewertung eingeht, ist elementar und keineswegs selbsterklärend. Nach Gleichung (2.7) gilt für den maximalen COP:

$$
C O P_{\max }=\frac{T_{\mathrm{H}}}{T_{\mathrm{H}}-T_{\mathrm{N}}}
$$

Der maximale COP wird unter der Annahme berechnet, dass sowohl die Wärmeaufnahme als auch die Wärmeabgabe isotherm stattfindet. Um den maximalen COP eines realen Prozesses errechnen zu können, muss daher festgelegt werden, welche Temperaturen in die Bestimmungsgleichung des maximalen COPs eingesetzt werden. In der vorliegenden Arbeit werden die Temperaturen wie folgt festgelegt:

- Zur Berechnung der hohen Temperatur $T_{\mathrm{H}}$ wird die Temperatur der Senke nach dem Kondensator um $\Delta T_{\mathrm{WT}}$ erhöht.

- Zur Berechnung der niedrigen Temperatur $T_{\mathrm{N}}$ wird die Temperatur der Quelle nach dem Verdampfer um $\Delta T_{\mathrm{WT}}$ verringert.

Daraus folgt für die Temperaturen $T_{\mathrm{H}}$ und $T_{\mathrm{N}}$ :

$$
\begin{aligned}
& T_{\mathrm{H}}=T_{\mathrm{S}}+\Delta T_{\mathrm{WT}} \\
& T_{\mathrm{N}}=T_{\mathrm{Q}}-\Delta T_{\mathrm{WT}}
\end{aligned}
$$


Zur besseren Nachvollziehbarkeit zeigt Abbildung 3.1, an welcher Stelle des Wärmepumpenkreislauf sich die Temperaturen $T_{\mathrm{S}}$ und $T_{\mathrm{Q}}$ befinden.

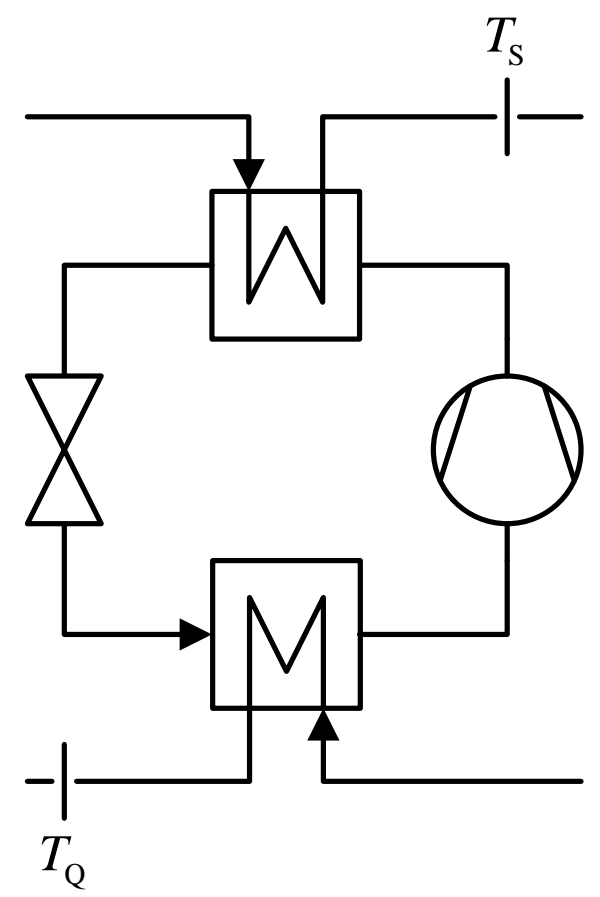

Abbildung 3.1: Bestimmung von $T_{\mathrm{Q}}$ und $T_{\mathrm{S}}$ am Wärmepumpenkreislauf

Ergänzung: Warum die Temperatur der Quelle nach dem Verdampfer für die Berechnung von $T_{\mathrm{N}}$ und die Temperatur der Senke nach dem Kondensator für die Berechnung von $T_{\mathrm{H}}$ herangezogen wird, bedarf einer gesonderten Erklärung, da diese Größen einen erheblichen Einfluss auf den maximalen COP und damit auf das gesamte Bewertungsergebnis haben und zusätzlich nicht sofort ersichtlich ist, warum diese örtlichen Definitionen an den beschriebenen Stellen des Wärmepumpenkreislaufs getätigt werden.

Es darf an dieser Stelle auch nicht verschwiegen werden, dass die nach Abbildung 3.1 eingeführte örtliche Festlegung der Temperaturen $T_{\mathrm{S}}$ und $T_{\mathrm{Q}}$ aus Sicht der Anwendung auf den ersten Blick wenig intuitiv ist und daher zu Verwirrung führen kann. Eine mögliche Erklärung für etwaige Verwirrungen geben die folgenden Ausführungen:

Zur Erhebung des wirtschaftlichen Potentials für die Integration einer Wärmepumpe, werden im ersten Schritt mögliche Quellen und Senken identifiziert. Das Ergebnis dieses Identifikationsprozesses ist zumeist:

1. Das Temperaturniveau auf dem die Prozesse mit thermischer Energie versorgt werden.

2. Das Temperaturniveau bei dem etwaige Quellen verfügbar sind. 
Das erste Ergebnis ist unproblematisch. Bei einem Prozess i folgt daraus beispielsweise die Temperatur $T_{\mathrm{Si}}$. In diesem Punkt entspricht der intuitive Ansatz den Erfordernissen des Berechnungskonzepts. Das zweite Ergebnis ist wesentlich problematischer, da die Temperatur auf dem etwaige Quellen verfügbar sind, nicht der Temperatur entspricht, die für das Berechnungskonzept benötigt wird.

Eine Frage, die in diesem Kontext häufig gestellt wird, ist, warum die Temperatur $T_{\mathrm{Q}}$ nicht einfach anders definiert wird. Bevor eine Wärmepumpe nicht zumindest theoretisch konzipiert wurde, ist die Temperatur der Quelle nach ihrer "Nutzung" ja ohnehin nicht bekannt. Was spräche dagegen, die Temperatur $T_{\mathrm{Q}}$ als genau jene Temperatur zu definieren, die die Quelle vor dem Verdampfer aufweist?

Die Beantwortung dieser Fragestellung ist wichtig für das Gesamtverständnis des Bewertungskonzepts. Ihr wird daher an dieser Stelle separater Raum gewährt:

Nach Gleichung (2.7) gilt für den maximalen COP:

$$
C O P_{\max }=\frac{T_{\mathrm{H}}}{T_{\mathrm{H}}-T_{\mathrm{N}}}
$$

Es gilt daher Temperaturen zu definieren, die im idealen Vergleichsprozess der Temperatur der reversiblen isothermen Wärmeaufnahme $T_{\mathrm{N}}$ und der reversiblen isothermen Wärmeabgabe $T_{\mathrm{H}}$, möglichst nahe kommen.

Abbildung 3.2 zeigt den idealisierten Temperaturverlauf im Kondensator eines theoretischen Wärmepumenkreislaufs. Es ist zu erkennen, dass die Temperatur der Wärmesenke am Austritt des Kondensators, erhöht um eine charakteristische Temperaturdifferenz, eine praktikable Näherung der Temperatur $T_{\mathrm{H}}$ darstellt. Das ist genau die Näherung, die in Gleichung (3.1) für Temperatur $T_{\mathrm{H}}$ formuliert wurde:

$$
T_{\mathrm{H}}=\underbrace{T_{\mathrm{S}}}_{\text {am Austritt }}+\Delta T_{\mathrm{WT}}
$$

Abbildung 3.3 zeigt den idealisierten Temperaturverlauf im Verdampfer eines theoretischen Wärmepumenkreislaufs. Aus dem Diagramm ist ablesbar, dass nur die Temperatur der Wärmequelle am Austritt des Kondensators, reduziert um eine charakteristische Temperaturdifferenz, als gute Approximation der Temperatur $T_{\mathrm{N}}$ verwendet werden kann. Daher wird wie in Gleichung (3.1) beschrieben, die niedrige Temperatur $T_{\mathrm{N}}$ wie folgt berechnet:

$$
T_{\mathrm{N}}=\underbrace{T_{\mathrm{Q}}}_{\text {am Austritt }}-\Delta T_{\mathrm{WT}}
$$

Zusammenfassend lässt sich feststellen, dass die gewählte örtliche Definition der Temperaturen $T_{\mathrm{Q}}$ und $T_{\mathrm{S}}$ am Wärmepumpenkreislauf (vgl. Abbildung 3.1) gewährleistet, dass diese Temperaturen durch Subtraktion bzw. Addition einer charakteristischen Temperaturdifferenz für die Berechnung des maximalen COPs herangezogen werden können. 


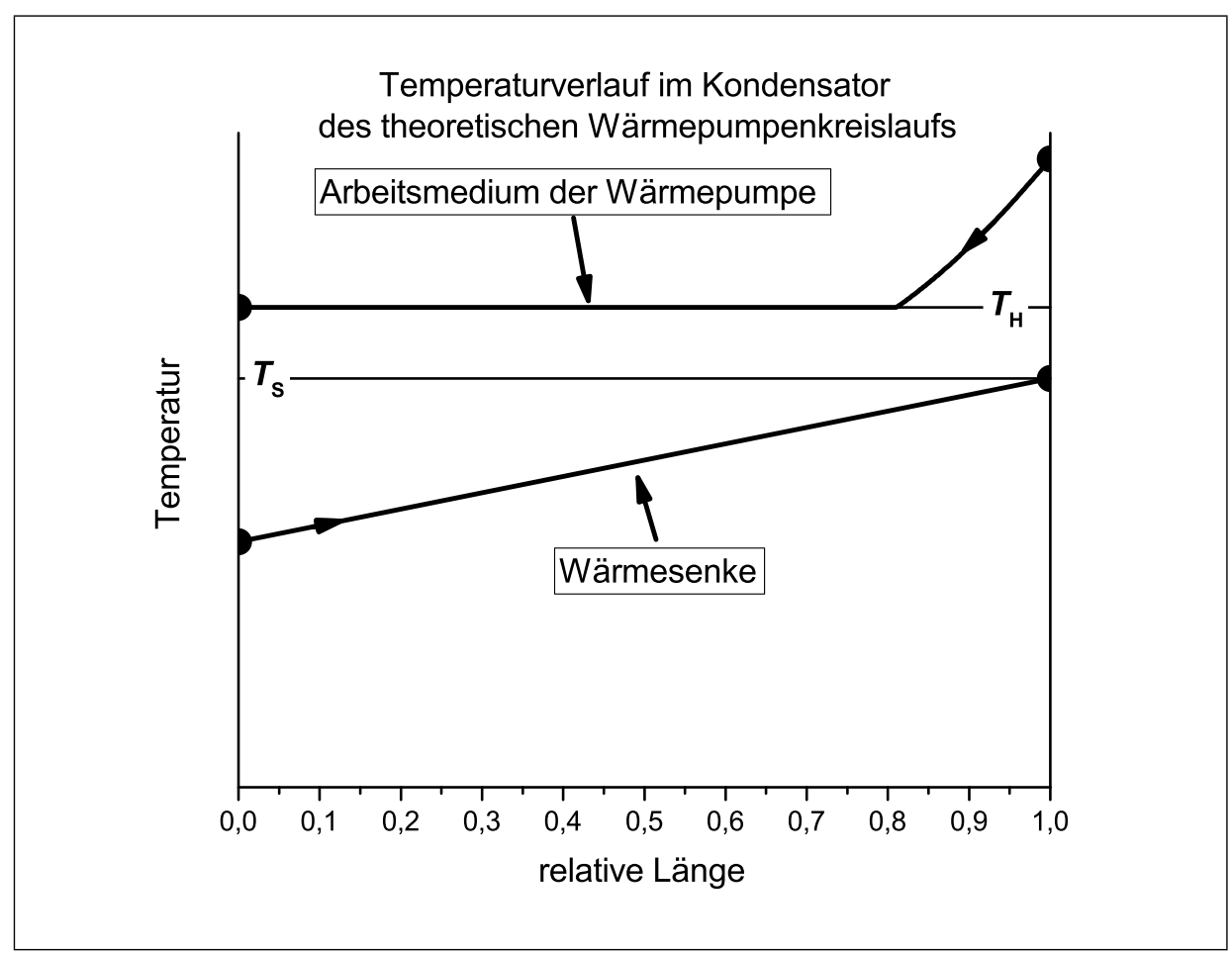

Abbildung 3.2: Idealisierter Temperaturverlauf im Kondensator eines theoretischen Wärmepumpenkreislaufs

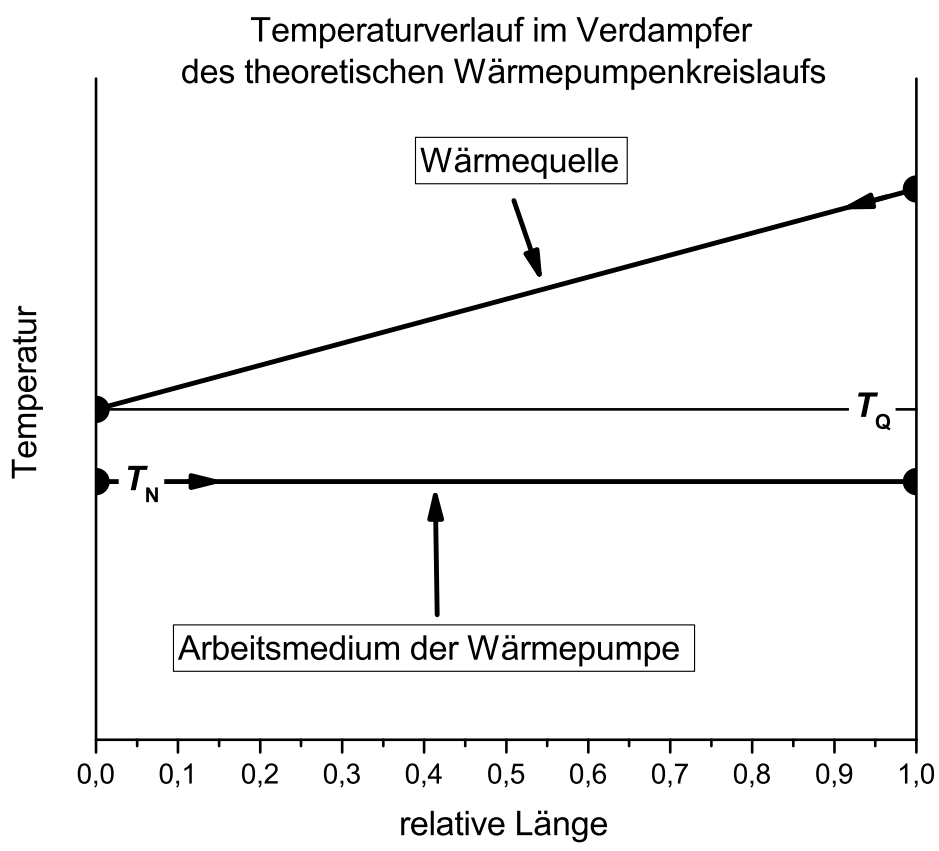

Abbildung 3.3: Idealisierter Temperaturverlauf im Verdampfer eines theoretischen Wärmepumpenkreislaufs 
Anmerkung: Häufig ist im Kontext der Wärmepumpe von der Wärmenutzungstemperatur die Rede. Diese entspricht im vorliegenden Dokument der Temperatur $T_{\mathrm{S}}$.

\section{Gütegrad}

Nach Formel (2.10) ist der Gütegrad eines Wärmepumpenprozesses definiert, als der Quotient aus dem realen COP mit dem maximalen COP:

$$
\eta_{\mathrm{WP}}=\frac{C O P_{\text {real }}}{C O P_{\max }}
$$

Für den Gütegrad wird im Folgenden angenommen:

- Der Gütegrad, in seiner Funktion als Gesamtwirkungsgrad [4], berücksichtigt jegliche Verluste, die auf der Seite des Kältemittelkreislaufs auftreten.

- Der Gütegrad, in seiner Funktion als eine charakteristische Größe für die Qualität eines Wärmepumpenkreislaufs, beschreibt, in welchem Umfang eine reale Anlage, im Vergleich zu einer idealen Anlage, einen Wärmestrom bei niedriger Temperatur aufnehmen und unter Zufuhr von Energie bei einer höheren Temperatur abgeben kann. Diese Fähigkeit hängt per Annahme nicht von der Prozessführung der äußeren Ströme (d.h. der Parameter der Quelle und der Senke) ab und wird daher als konstant angenommen.

Anmerkung: Die Rolle des Gütegrads ist auf den ersten Blick nicht immer sofort ersichtlich, da er im Rahmen einer ersten Evaluierung des Potentials für die Integration einer Wärmepumpe zumeist abgeschätzt wird. Im Folgenden wird daher eine kurze Ergänzung zum Gütegrad gegeben:

Eine Stärke des Gütegrads ist, dass er für eine reale Anlage, unter Verwendung der bisher in Kapitel 3.2 getätigten Annahmen und Definitionen, leicht zu ermitteln ist. Der reale COP ist nach Gleichung (2.9) gegeben mit:

$$
C O P_{\text {real }}=\frac{\dot{Q}_{34}^{\mathrm{r}}}{\dot{W}_{23}^{\mathrm{r}}}
$$

Da der Gütegrad alle Verluste auf der Seite des Kältemittelkreislaufs berücksichtigt, entspricht $\dot{W}_{23}^{\mathrm{r}}$ die dem Verdichter zugeführte, und messtechnisch leicht erhebbare, elektrische Leistung. Der Term $\dot{Q}_{34}^{\mathrm{r}}$, welcher den Nutzen darstellt, lässt sich bei bekanntem Massenstrom der Senke durch eine Temperaturmessung der Senke vor und nach dem Kondensator feststellen. Dadurch ist ebenfalls $T_{\mathrm{S}}$ bestimmt. Wird nun zu guter Letzt $T_{\mathrm{Q}}$, definiert als die Temperatur der Quelle nach dem Verdampfer, durch eine Messung ermittelt, dann sind, bei Annahme eines charakteristischen $\Delta T_{\mathrm{WT}}$, alle Größen die für die Berechnung des Gütegrads notwendig sind, bekannt. 
Einmal an der realen Anlage festgestellt, kann der Gütegrad als konstante charakteristische Größe des Wärmepumpenkreislaufs angesehen werden. Dadurch ist es möglich, die Wärmepumpe als eine Art Blackbox darzustellen und ihre Leistungsfähigkeit an verschiedensten Konstellationen aus Quelle und Senke zu untersuchen.

\subsection{Technische Kennzahlen}

In diesem Kapitel werden bekannte und neue technische Kennzahlen unter Verwendung der in Kapitel 3.2 getätigten Annahmen und Definitionen angeschrieben bzw. eingeführt. Für die Indizes gilt:

- Index H hochgestellt

- Index K hochgestellt

- Index $\mathrm{H}+\mathrm{K}$ hochgestellt

- Index $\mathrm{N}$ tiefgestellt

- Index Kv hochgestellt
Heizen bzw. im Heizbetrieb

Kühlen bzw. im Kühlbetrieb

Heizen und Kühlen bzw. im Heiz- und Kühltrieb

Nutzen (gilt nur für Wärme bzw. Wärmeströme)

Vergleichsprozess - Kühlen bzw. im Kühlbetrieb

Anmerkung: Der Einsatz einer Wärmepumpe im Heizbetrieb wird im Folgenden auch als im "einfachen" Betrieb, der Einsatz im Heiz- und Kühlbetrieb als im "kombinierten" Betrieb beschrieben.

\subsubsection{Maximaler COP im Heizbetrieb}

Der maximale COP im Heizbetrieb lässt sich nach Gleichung (2.7) unter Verwendung der in Gleichung (3.1) definierten Temperaturvorschriften wie folgt anschreiben:

$$
C O P_{\max }^{\mathrm{H}}=\frac{T_{\mathrm{H}}}{T_{\mathrm{H}}-T_{\mathrm{N}}}=\frac{T_{\mathrm{S}}+\Delta T_{\mathrm{WT}}}{T_{\mathrm{S}}+2 \Delta T_{\mathrm{WT}}-T_{\mathrm{Q}}}
$$

Das Ergebnis aus Gleichung (3.2) ist im Bezug auf die Zielsetzung der vorliegenden Arbeit problematisch, da mit $\Delta T_{\mathrm{WT}}$ bereits in der ersten Formel des Bewertungskonzepts eine Größe steht, die im Vorfeld festgelegt werden muss.

Um Gleichung (3.2) in eine allgemeinere Form überführen zu können, werden zwei Größen eingeführt: 
- Maximaler COP im Heizbetrieb bei $\Delta T_{\mathrm{WT}}=0{ }^{\circ} \mathrm{C}$ :

$$
\begin{aligned}
& C O P_{0}^{\mathrm{H}}:=\left.C O P_{\max }^{\mathrm{H}}\right|_{\Delta T_{\mathrm{WT}}=0} \\
& \longrightarrow C O P_{0}^{\mathrm{H}}=\frac{T_{\mathrm{S}}}{T_{\mathrm{S}}-T_{\mathrm{Q}}}
\end{aligned}
$$

- Temperaturfaktor:

$$
X_{\mathrm{T}}
$$

Unter Verwendung des noch unbestimmten Temperaturfaktors $X_{\mathrm{T}}$ soll gelten:

$$
C O P_{\max }^{\mathrm{H}}=X_{\mathrm{T}} C O P_{0}^{\mathrm{H}}
$$

unter der Nebenbedingung, dass sowohl $C O P_{0}^{\mathrm{H}}$ als auch $X_{\mathrm{T}}$ so bestimmt werden, dass es möglich ist, diese Größen in Diagrammen darzustellen bzw. aus Diagrammen abzulesen.

\section{Ermittlung eines Zusammenhangs für $\boldsymbol{X}_{\mathbf{T}}$}

Wird in Gleichung (3.5) die Größe $C O P_{\max }^{\mathrm{H}}$ unter Verwendung von Gleichung (3.2) ausgedrückt, so folgt:

$$
\begin{array}{r}
C O P_{\text {max }}^{\mathrm{H}}=X_{\mathrm{T}} C O P_{0}^{\mathrm{H}} \\
\longrightarrow \frac{T_{\mathrm{S}}+\Delta T_{\mathrm{WT}}}{T_{\mathrm{S}}+2 \Delta T_{\mathrm{WT}}-T_{\mathrm{Q}}}=X_{\mathrm{T}} C O P_{0}^{\mathrm{H}}
\end{array}
$$

Mit der Definition:

$$
\Delta T_{\mathrm{SQ}}:=T_{\mathrm{S}}-T_{\mathrm{Q}}
$$

lässt sich $T_{\mathrm{S}}$ durch:

$$
\begin{aligned}
C O P_{0}^{\mathrm{H}} & =\frac{T_{\mathrm{S}}}{T_{\mathrm{S}}-T_{\mathrm{Q}}} \\
T_{\mathrm{S}} & =\operatorname{COP}_{0}^{\mathrm{H}}\left(T_{\mathrm{S}}-T_{\mathrm{Q}}\right) \\
\longrightarrow T_{\mathrm{S}} & =\operatorname{COP}_{0}^{\mathrm{H}} \Delta T_{\mathrm{SQ}}
\end{aligned}
$$

ausdrücken.

Das Resultat aus Gleichung (3.8) sowie die Definition aus Gleichung (3.7) in Gleichung (3.6) eingesetzt, erlaubt die folgenden Umformungen: 


$$
\begin{aligned}
X_{\mathrm{T}} C O P_{0}^{\mathrm{H}} & =\frac{T_{\mathrm{S}}+\Delta T_{\mathrm{WT}}}{T_{\mathrm{S}}+2 \Delta T_{\mathrm{WT}}-T_{\mathrm{Q}}} \\
\longrightarrow X_{\mathrm{T}} C O P_{0}^{\mathrm{H}} & =\frac{C O P_{0}^{\mathrm{H}} \Delta T_{\mathrm{SQ}}+\Delta T_{\mathrm{WT}}}{\Delta T_{\mathrm{SQ}}+2 \Delta T_{\mathrm{WT}}} \\
X_{\mathrm{T}} & =\frac{1}{C O P_{0}^{\mathrm{H}}} \cdot\left(\frac{C O P_{0}^{\mathrm{H}} \Delta T_{\mathrm{SQ}}+\Delta T_{\mathrm{WT}}}{\Delta T_{\mathrm{SQ}}+2 \Delta T_{\mathrm{WT}}}\right)=\frac{C O P_{0}^{\mathrm{H}} \Delta T_{\mathrm{SQ}}+\Delta T_{\mathrm{WT}}}{C O P_{0}^{\mathrm{H}} \Delta T_{\mathrm{SQ}}+2 C O P_{0}^{\mathrm{H}} \Delta T_{\mathrm{WT}}} \\
X_{\mathrm{T}} & =\frac{C O P_{0}^{\mathrm{H}} \Delta T_{\mathrm{SQ}}+2 C O P_{0}^{\mathrm{H}} \Delta T_{\mathrm{WT}}-2 C O P_{0}^{\mathrm{H}} \Delta T_{\mathrm{WT}}+\Delta T_{\mathrm{WT}}}{C O P_{0}^{\mathrm{H}} \Delta T_{\mathrm{SQ}}+2 C O P_{0}^{\mathrm{H}} \Delta T_{\mathrm{WT}}} \\
X_{\mathrm{T}} & =1+\frac{\Delta T_{\mathrm{WT}}}{C O P_{0}^{\mathrm{H}}}\left(\frac{1-2 C O P_{0}^{\mathrm{H}}}{\Delta T_{\mathrm{SQ}}+2 \Delta T_{\mathrm{WT}}}\right)
\end{aligned}
$$

Es wird die dimensionslose Temperaturdifferenz $\widehat{\Delta T}$ eingeführt:

$$
\begin{aligned}
\widehat{\Delta T}: & =\frac{\Delta T_{\mathrm{WT}}}{\Delta T_{\mathrm{SQ}}} \\
\longrightarrow \Delta T_{\mathrm{WT}} & =\widehat{\Delta T} \Delta T_{\mathrm{SQ}}
\end{aligned}
$$

Wird der Ausdruck für $\Delta T_{\mathrm{WT}}$ aus Gleichung (3.10) in Gleichung (3.9) eingesetzt, so folgt daraus:

$$
\begin{aligned}
X_{\mathrm{T}} & =1+\frac{\Delta T_{\mathrm{WT}}}{C O P_{0}^{\mathrm{H}}}\left(\frac{1-2 C O P_{0}^{\mathrm{H}}}{\Delta T_{\mathrm{SQ}}+2 \Delta T_{\mathrm{WT}}}\right) \\
\longrightarrow X_{\mathrm{T}} & =1+\frac{\widehat{\Delta T} \Delta T_{\mathrm{SQ}}}{C O P_{0}^{\mathrm{H}}}\left(\frac{1-2 C O P_{0}^{\mathrm{H}}}{\Delta T_{\mathrm{SQ}}+2 \widehat{\Delta T} \Delta T_{\mathrm{SQ}}}\right)
\end{aligned}
$$

Was schlussendlich den gesuchten Zusammenhang für $X_{\mathrm{T}}$ ergibt:

$$
X_{\mathrm{T}}=1+\frac{\widehat{\Delta T}}{C O P_{0}^{\mathrm{H}}}\left(\frac{1-2 C O P_{0}^{\mathrm{H}}}{1+2 \widehat{\Delta T}}\right)
$$




\section{Ableitung von Diagrammen für $\widehat{\Delta T}$}

Zur Berechnung von $\widehat{\Delta T}$ kann der Zusammenhang aus Gleichung (3.10) herangezogen werden:

$$
\widehat{\Delta T}=\frac{\Delta T_{\mathrm{WT}}}{\Delta T_{\mathrm{SQ}}}
$$

Abbildung 3.4 und Abbildung 3.5 zeigen $\widehat{\Delta T}$ als Funktion von $\Delta T_{\mathrm{SQ}}$ für konstante Werte von $\Delta T_{\mathrm{WT}}$. Der Unterschied zwischen Abbildung 3.4 und Abbildung 3.5 ist das jeweilige Intervall von $\Delta T_{\mathrm{SQ}}$. Diese Trennung ist notwendig gewesen, da ansonsten ohne logarithmische Darstellung der Ordinatenachse ein Ablesen nur schwer möglich gewesen wäre. Auf eine graphische Darstellung von $\Delta T_{\mathrm{SQ}}$ als Funktion von $T_{\mathrm{S}}$ und $T_{\mathrm{Q}}$ ist verzichtet worden, um die Anzahl an Diagrammen, die für das gesamte Bewertungsschema benötigt werden, nicht unnötig in die Höhe zu treiben.

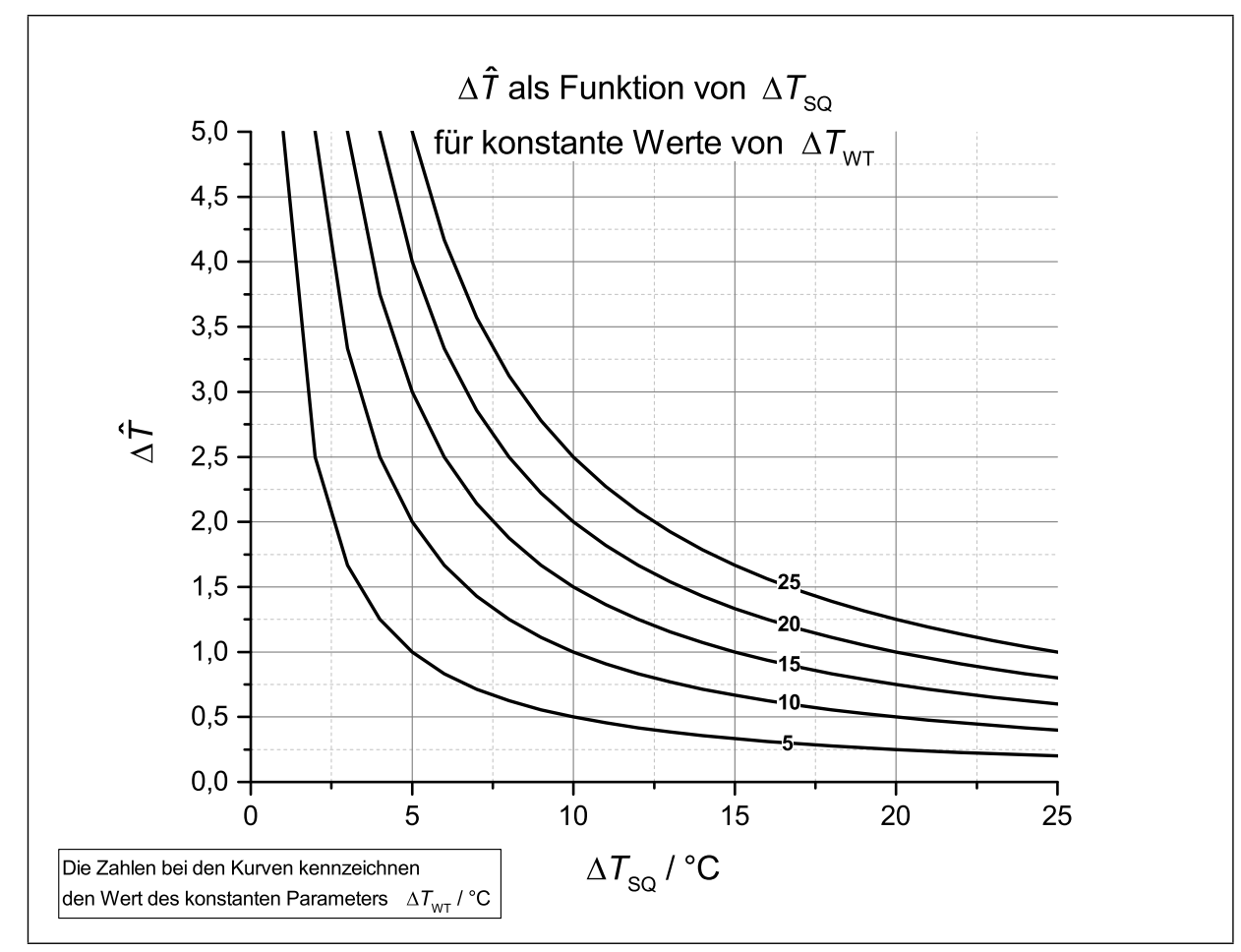

Abbildung 3.4: $\widehat{\Delta T}$ als Funktion von $\Delta T_{\mathrm{SQ}}$ für konstante Werte von $\Delta T_{\mathrm{WT}}$ im Intervall $0{ }^{\circ} \mathrm{C} \leq \Delta T_{\mathrm{SQ}} \leq 25^{\circ} \mathrm{C}$ 


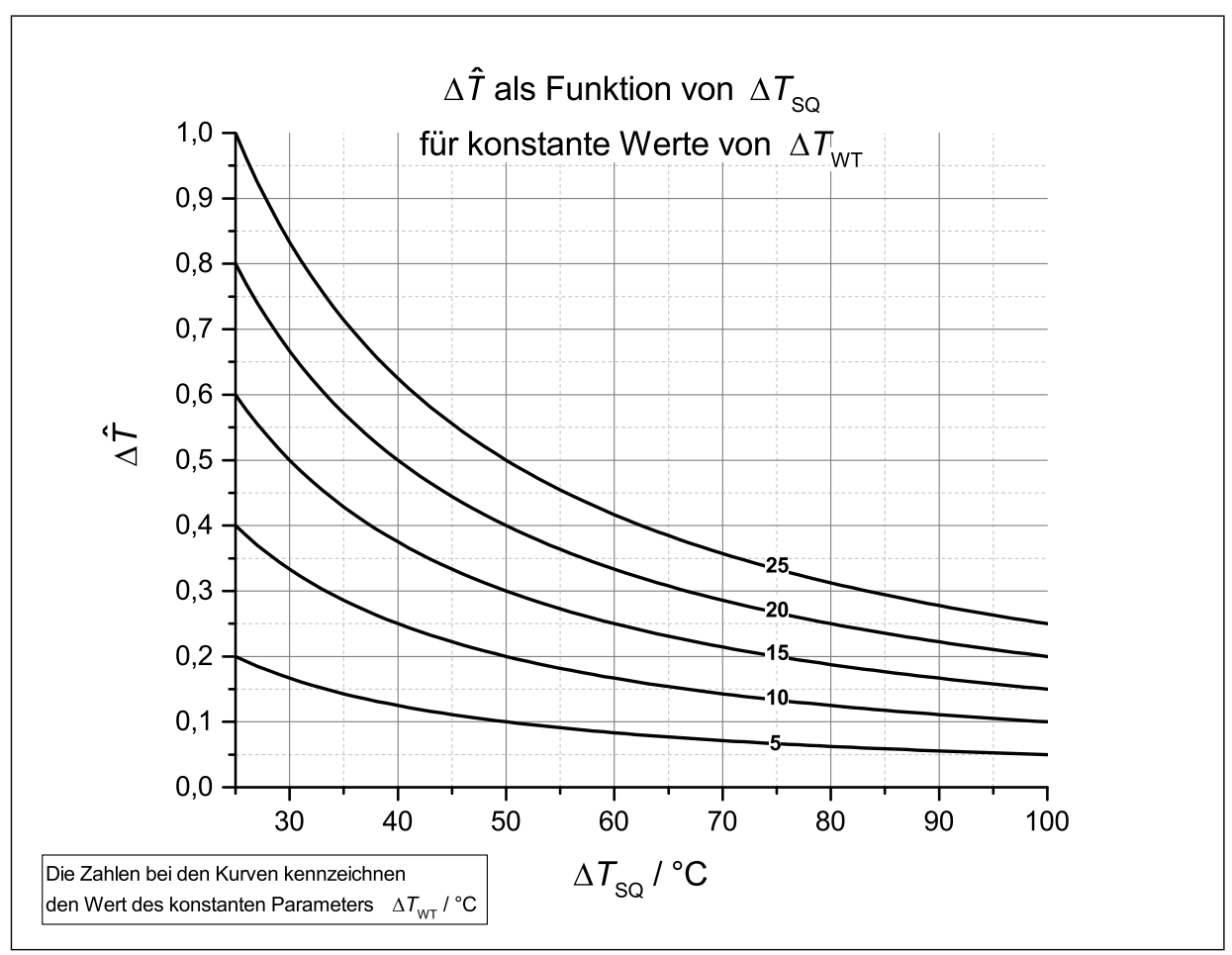

Abbildung 3.5: $\widehat{\Delta T}$ als Funktion von $\Delta T_{\mathrm{SQ}}$ für konstante Werte von $\Delta T_{\mathrm{WT}}$ im Intervall $25^{\circ} \mathrm{C} \leq \Delta T_{\mathrm{SQ}} \leq 100{ }^{\circ} \mathrm{C}$

\section{Hinweise zu den Diagrammen zur Ermittlung von $\widehat{\Delta T}$ :}

- Auf der Abszisse ist $\Delta T_{\mathrm{SQ}}$ aufgetragen. Die Kurven zeigen $\widehat{\Delta T}$ bei einem konstanten $\Delta T_{\mathrm{WT}}$.

- Der Ableseprozess lässt sich wie folgt darstellen:

- Im ersten Schritt wird $\Delta T_{\mathrm{SQ}}$ aus $T_{\mathrm{S}}$ und $T_{\mathrm{Q}}$ errechnet. Daran anschließend folgt die einmalige Festlegung von $\Delta T_{\mathrm{WT}}$.

- Zur Bestimmung von $\widehat{\Delta T}$ wird gedanklich eine Vertikale durch das errechnete $\Delta T_{\mathrm{SQ}}$ gelegt und mit der zum gewählten $\Delta T_{\mathrm{WT}}$ passenden Kurve zum Schnitt gebracht. Für den so ermittelten Schnittpunkt kann $\widehat{\Delta T}$ abgelesen werden.

- Für ein $\Delta T_{\mathrm{SQ}}$ von $10{ }^{\circ} \mathrm{C}$ lässt sich beispielsweise bei einem $\Delta T_{\mathrm{SW}}$ von $10{ }^{\circ} \mathrm{C}$ die gesuchte Größe $\widehat{\Delta T}=1$ ermitteln. 


\section{Erstellung von Diagrammen für $C O P_{0}^{\mathrm{H}}$}

Es existieren mehrere Möglichkeiten um $C O P_{0}^{\mathrm{H}}$ in Diagrammen abzubilden. Denkbare wäre zum Beispiel die Einführung einer neuen Kennzahl die stets kleiner als 1 ist:

$$
\hat{T}:=\frac{T_{\mathrm{Q}}}{T_{\mathrm{S}}}
$$

wodurch sich $C O P_{0}^{\mathrm{H}}$ in:

$$
C O P_{0}^{\mathrm{H}}=\frac{1}{1-\hat{T}}
$$

überführen ließe. In diesem Fall wäre $C O P_{0}^{\mathrm{H}}$ nur noch von einer Größe abhängig und die Darstellung könnte auf ein Diagramm beschränkt werden.

Aus Sicht des Anwenders ist jedoch festzustellen, dass die Temperaturen $T_{\mathrm{Q}}$ und $T_{\mathrm{S}}$ bzw. $\vartheta_{\mathrm{Q}}$ und $\vartheta_{\mathrm{S}}$ die maßgeblichen Größen bei der technischen Einbindung einer Wärmepumpe darstellen. Der graphischen Darstellung, welchen Einfluss verschiedene Werte von $T_{\mathrm{Q}}$ und $T_{\mathrm{S}}$ auf $C O P_{0}^{\mathrm{H}}$ haben und welcher Effekt sich in weiterer Folge daraus für die Bewertung der Leistungsfähigkeit einer Wärmepumpe ergeben kann, ist eine hohe Bedeutung zuzusprechen.

Es ist daher entschieden worden zwei Arten von Diagrammen einzuführen:

\section{Diagramme vom Typ 1:}

Darstellung von $C O P_{0}^{\mathrm{H}}$ als Funktion von $\vartheta_{\mathrm{Q}}$ für konstante Werte von $\vartheta_{\mathrm{S}}$ bzw. als Funktion von $\vartheta_{\mathrm{S}}$ für konstante Werte von $\vartheta_{\mathrm{Q}}$. Dazu zählen die Abbildungen 3.6 und 3.7 .

\section{Diagramme vom Typ 2:}

Darstellung von $\vartheta_{\mathrm{S}}$ als Funktion von $\vartheta_{\mathrm{Q}}$ für konstante Werte von $C O P_{0}^{\mathrm{H}}$. Die Abbildungen 3.8 ist vom Typ 2.

Anmerkung: $\mathrm{Ob}$ die Größe $C O P_{0}^{\mathrm{H}}$ die an sie gestellten Anforderungen erfüllen kann, wird in Kapitel 6.1 erneut aufgegriffen. Alternative Ansätze zur Bestimmung von $C O P_{\max }^{\mathrm{H}}$, die ohne $C O P_{0}^{\mathrm{H}}$ und $X_{\mathrm{T}}$ ihr Auskommen finden, werden in Kapitel 3.3.2 eingeführt. 


\section{Diagramme vom Typ 1:}

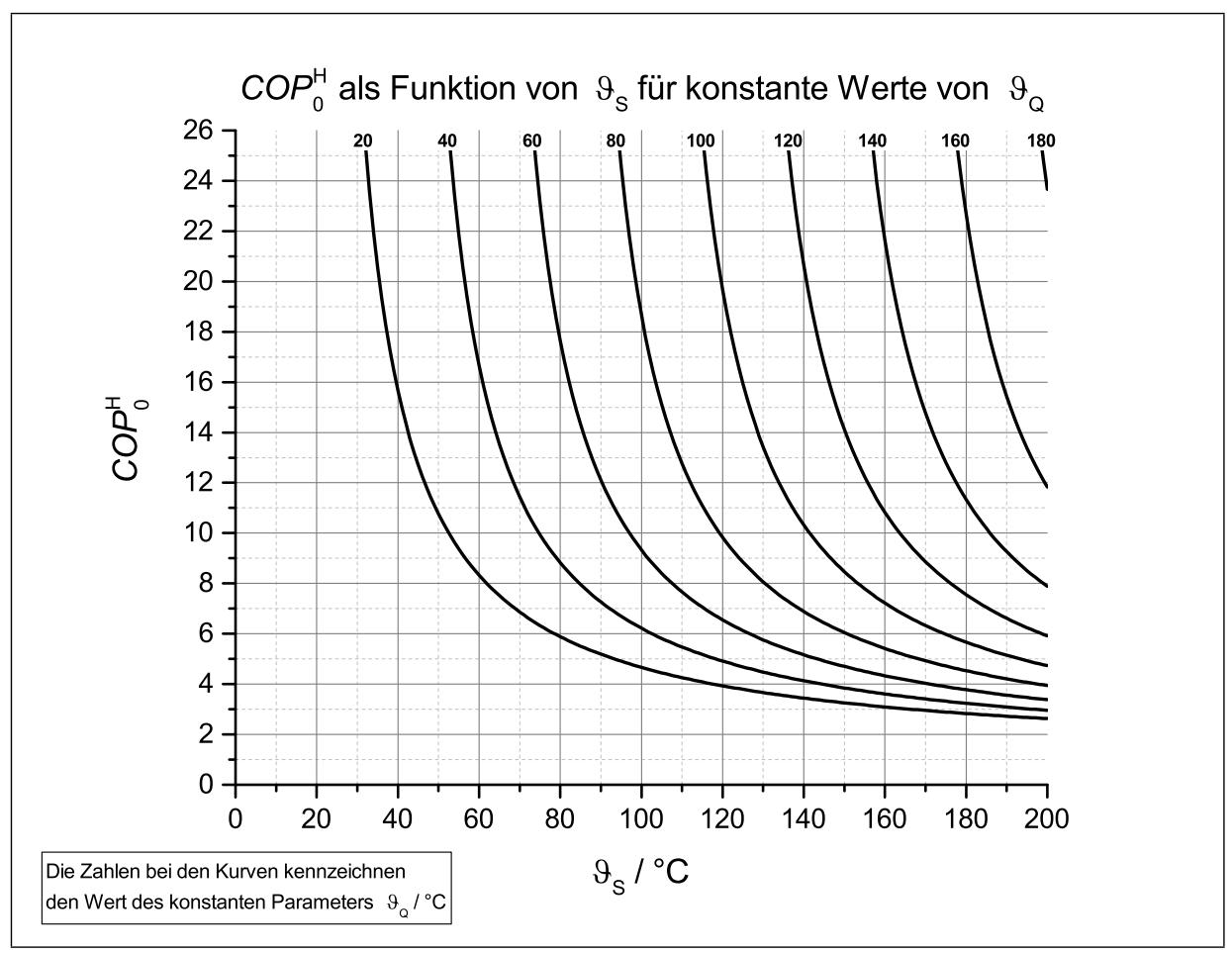

Abbildung 3.6: $C O P_{0}^{\mathrm{H}}$ als Funktion von $\vartheta_{\mathrm{S}}$ für konstante Werte von $\vartheta_{\mathrm{Q}}$

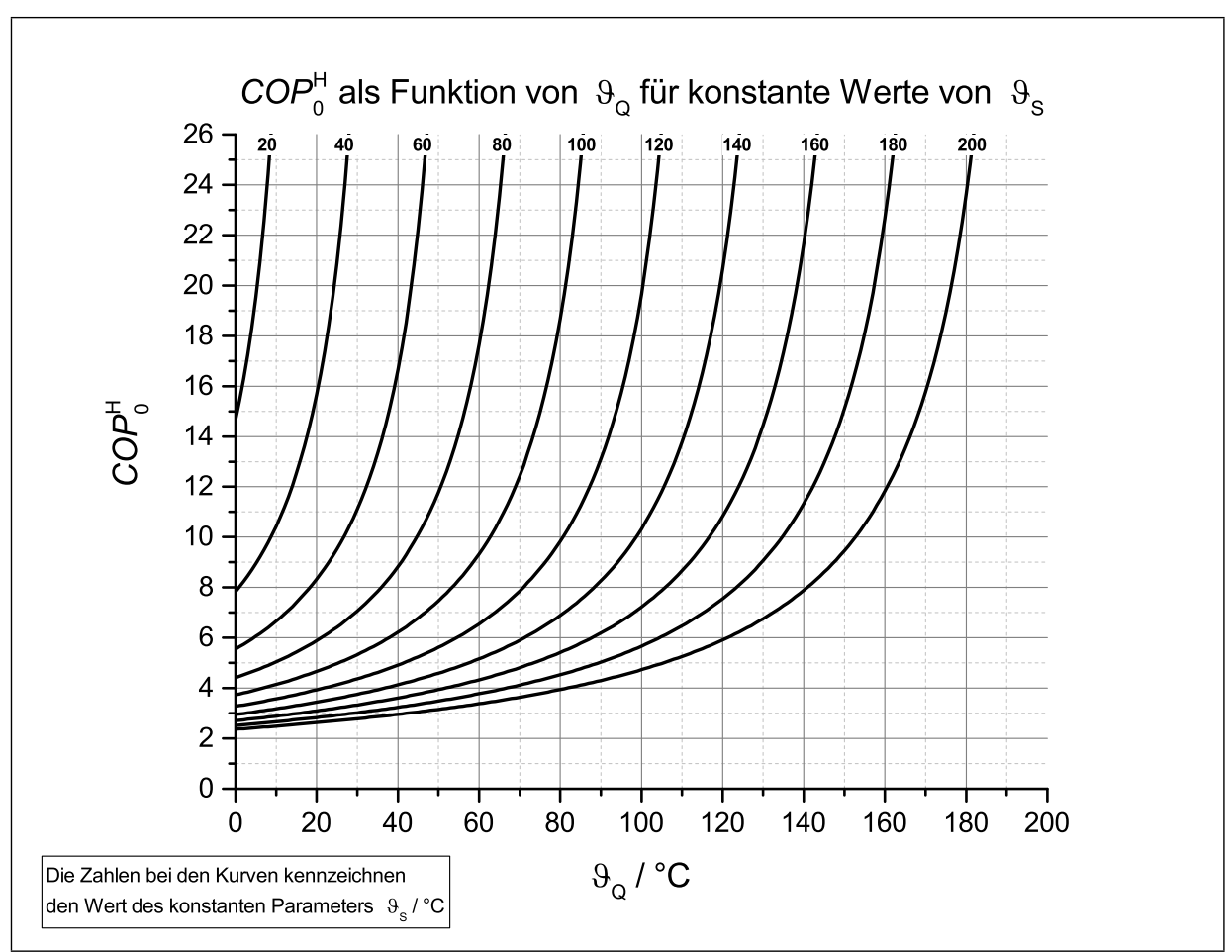

Abbildung 3.7: $C O P_{0}^{\mathrm{H}}$ als Funktion von $\vartheta_{\mathrm{Q}}$ für konstante Werte von $\vartheta_{\mathrm{S}}$ 


\section{Hinweise zu den Diagrammen vom Typ 1:}

- Auf der Abszisse in Abbildung 3.6 ist $\vartheta_{\mathrm{S}}$ aufgetragen. Die Kurven zeigen $C O P_{0}^{\mathrm{H}}$ bei konstanten $\vartheta_{\mathrm{Q}}$. Für Abbildung 3.7 gilt das gleiche, nur dass $\vartheta_{\mathrm{S}}$ in diesem Fall auf der Ordinate und $\vartheta_{\mathrm{S}}$ auf der Abszisse aufgetragen ist.

- Diagramme vom Typ 1 dienen der Ermittlung von $C O P_{0}^{\mathrm{H}}$ für eine Kombination aus $\vartheta_{\mathrm{S}}$ und $\vartheta_{\mathrm{Q}}$. Der Ableseweg sei im Folgenden am Beispiel von $\vartheta_{\mathrm{S}}$ und $\vartheta_{\mathrm{Q}}=$ konstant beschrieben:

Es wird eine Vertikale durch $\vartheta_{\mathrm{S}}$ gelegt und mit der korrespondierenden Kurve für $\vartheta_{\mathrm{Q}}=$ konstant geschnitten. Für den ermittelten Schnittpunkt kann $C O P_{0}^{\mathrm{H}}$ abgelesen werden.

- Für $\vartheta_{\mathrm{S}}=100^{\circ} \mathrm{C}$ und $\vartheta_{\mathrm{Q}}=40^{\circ} \mathrm{C}$ lässt sich beispielsweise $C O P_{0}^{\mathrm{H}}=6,2$ ablesen. 


\section{Diagramme vom Typ 2:}

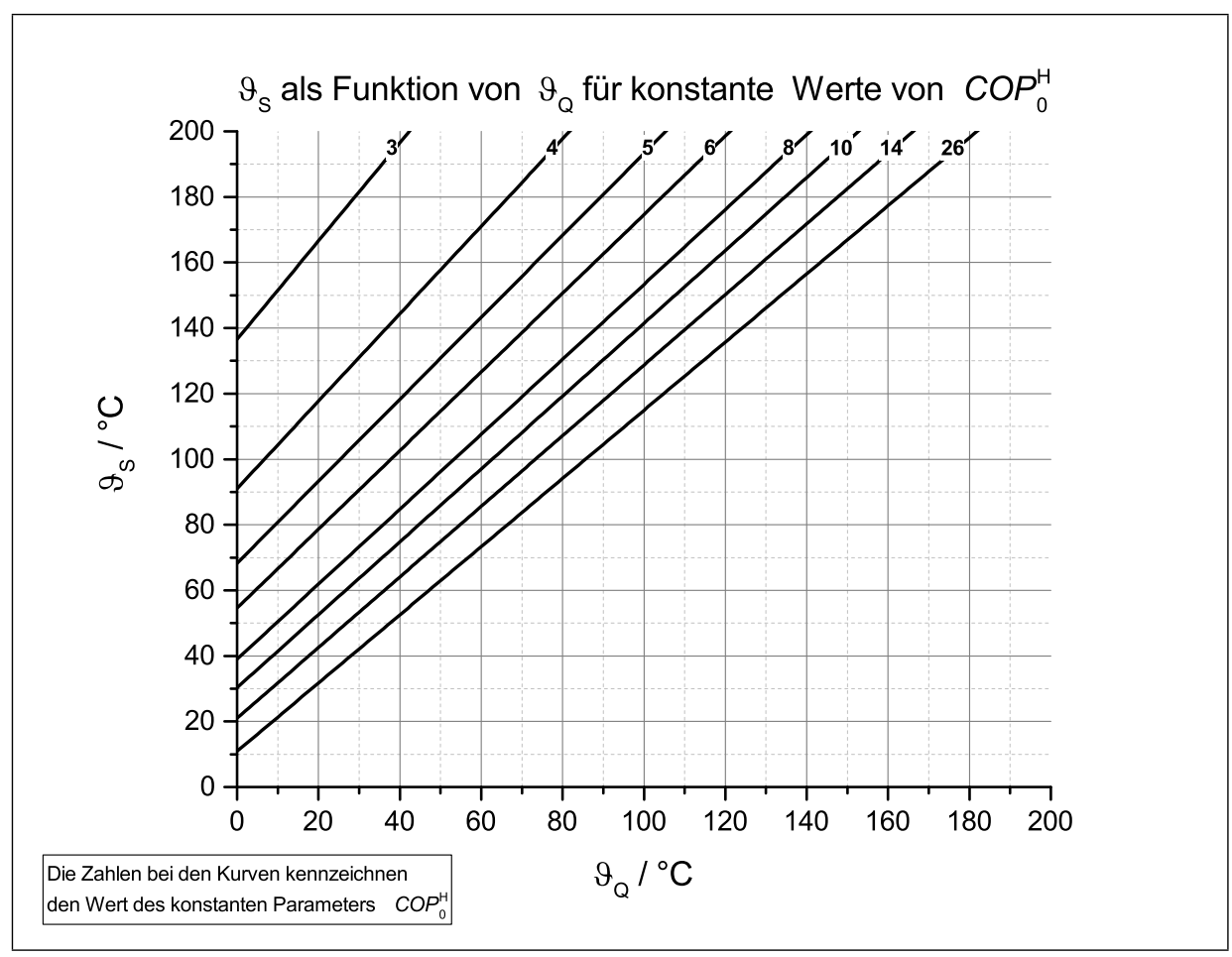

Abbildung 3.8: $\vartheta_{\mathrm{S}}$ als Funktion von $\vartheta_{\mathrm{Q}}$ im Intervall $0{ }^{\circ} \mathrm{C} \leq \vartheta_{\mathrm{S}} \leq 200{ }^{\circ} \mathrm{C}$ für konstante Werte von $C O P_{0}^{\mathrm{H}}$

\section{Hinweise zu den Diagrammen vom Typ 2:}

- Auf der Abszisse in Abbildung 3.8 ist $\vartheta_{\mathrm{Q}}$ aufgetragen. Die Kurven zeigen $\vartheta_{\mathrm{S}}$ für konstante Werte von $C O P_{0}^{\mathrm{H}}$.

- Diagramme vom Typ 2 dienen der Ermittlung von $\vartheta_{\mathrm{S}}$ bzw. $\vartheta_{\mathrm{Q}}$ bei einem gegebenen $C O P_{0}^{\mathrm{H}}$.

- Der Ableseweg sei im Folgenden am Beispiel von $\vartheta_{\mathrm{S}}$ und $C O P_{0}^{\mathrm{H}}=$ konstant beschrieben, verläuft bei Vorgabe von $\vartheta_{\mathrm{Q}}$ statt $\vartheta_{\mathrm{S}}$ jedoch analog:

Es wird eine Horziontale durch $\vartheta_{\mathrm{S}}$ gelegt und mit der korrespondierenden Kurve für $C O P_{0}^{\mathrm{H}}=$ konstant zum Schnitt gebracht. Durch den Schnittpunkt wird eine Vertikale gelegt, welche die Abszisse im gesuchten Punkt $\vartheta_{\mathrm{Q}}$ schneidet.

- So lässt sich $C O P_{0}^{\mathrm{H}}=4$ beispielsweise durch $\vartheta_{\mathrm{Q}}=60{ }^{\circ} \mathrm{C}$ und $\vartheta_{\mathrm{S}}=172{ }^{\circ} \mathrm{C}$ aber auch $\vartheta_{\mathrm{Q}}=20^{\circ} \mathrm{C}$ und $\vartheta_{\mathrm{S}}=118^{\circ} \mathrm{C}$ erreichen. 


\section{Erstellung von Diagrammen für $\boldsymbol{X}_{\mathrm{T}}$}

Zur Visualisierung von $X_{\mathrm{T}}$ wird der in Gleichung (3.12) aufgestellte Zusammenhang:

$$
X_{\mathrm{T}}=1+\frac{\widehat{\Delta T}}{C O P_{0}^{\mathrm{H}}}\left(\frac{1-2 C O P_{0}^{\mathrm{H}}}{1+2 \widehat{\Delta T}}\right)
$$

für verschiedene Werte von $C O P_{0}^{\mathrm{H}}$ und $\widehat{\Delta T}$ graphisch dargestellt. Siehe hierzu Abbildung 3.9:

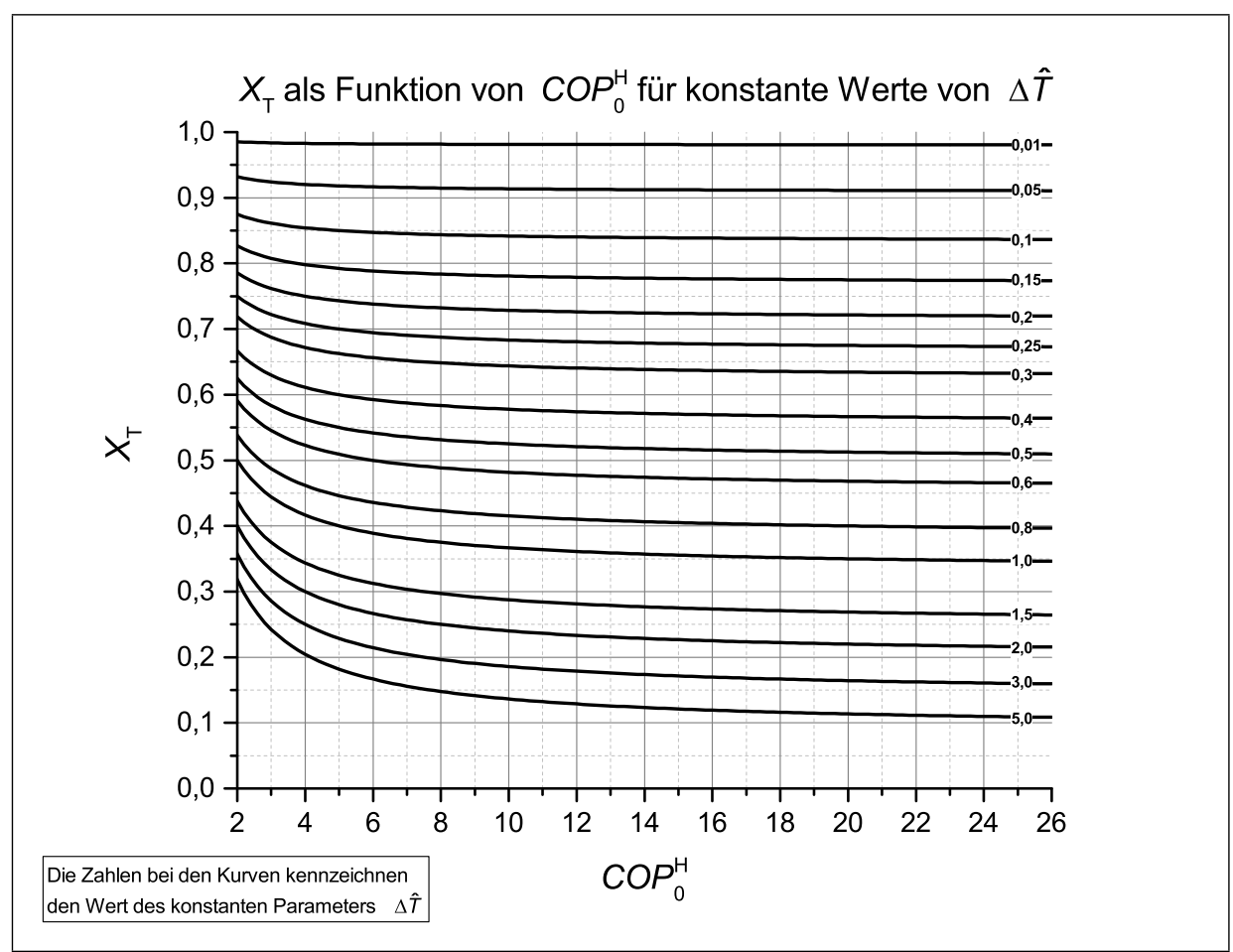

Abbildung 3.9: $X_{\mathrm{T}}$ als Funktion von $C O P_{0}^{\mathrm{H}}$ im Intervall $2 \leq C O P_{0}^{\mathrm{H}} \leq 26$ für konstante Werte von $\widehat{\Delta T}$

\section{Hinweise zu den Diagrammen zur Ermittlung von $\boldsymbol{X}_{\mathrm{T}}$}

- Auf der Abszisse in Abbildung 3.9 ist $C O P_{0}^{\mathrm{H}}$ im Intervall $2 \leq C O P_{0}^{\mathrm{H}} \leq 26$ aufgetragen. Das Intervall für $C O P_{0}^{\mathrm{H}}$ ist aus der Beobachtung (vgl. Abbildung 3.6 und 3.7) abgeleitet worden. Als konstanter Parameter wurde $\widehat{\Delta T}$ gewählt. Die inverse Darstellungsvariante, d.h. $X_{\mathrm{T}}$ als Funktion von $\widehat{\Delta T}$ für konstante Werte von $C O P_{0}^{\mathrm{H}}$ ist ebenfalls denkbar, jedoch in Form eines Diagrammes nicht leicht ablesbar. 
- Der Ableseweg wird im Folgenden am Beispiel von $C O P_{0}^{\mathrm{H}}$ und $\widehat{\Delta T}=$ konstant beschrieben:

Es wird eine Vertikale durch $C O P_{0}^{\mathrm{H}}$ gelegt und mit der korrespondierenden Kurve für $\widehat{\Delta T}=$ konstant geschnitten. Für den ermittelten Schnittpunkt kann $X_{\mathrm{T}}$ abgelesen werden.

- Für $C O P_{0}^{\mathrm{H}}=6$ und $\widehat{\Delta T}=0,6$ lässt sich beispielsweise $X_{\mathrm{T}}=0,5$ ermitteln.

\section{Erstellung von Diagrammen für $C_{\text {OPax }}^{\mathrm{H}}$}

Die Berechnungsvorschrift für $C O P_{\max }^{\mathrm{H}}$ ist durch:

$$
C O P_{\max }^{\mathrm{H}}=X_{\mathrm{T}} C O P_{0}^{\mathrm{H}}
$$

gegeben. Bei der Wahl von $X_{\mathrm{T}}$ als konstanten Parameter lässt sich $C O P_{\text {max }}^{\mathrm{H}}$, wie Abbildung 3.10 und 3.11 gezeigt, darstellen:

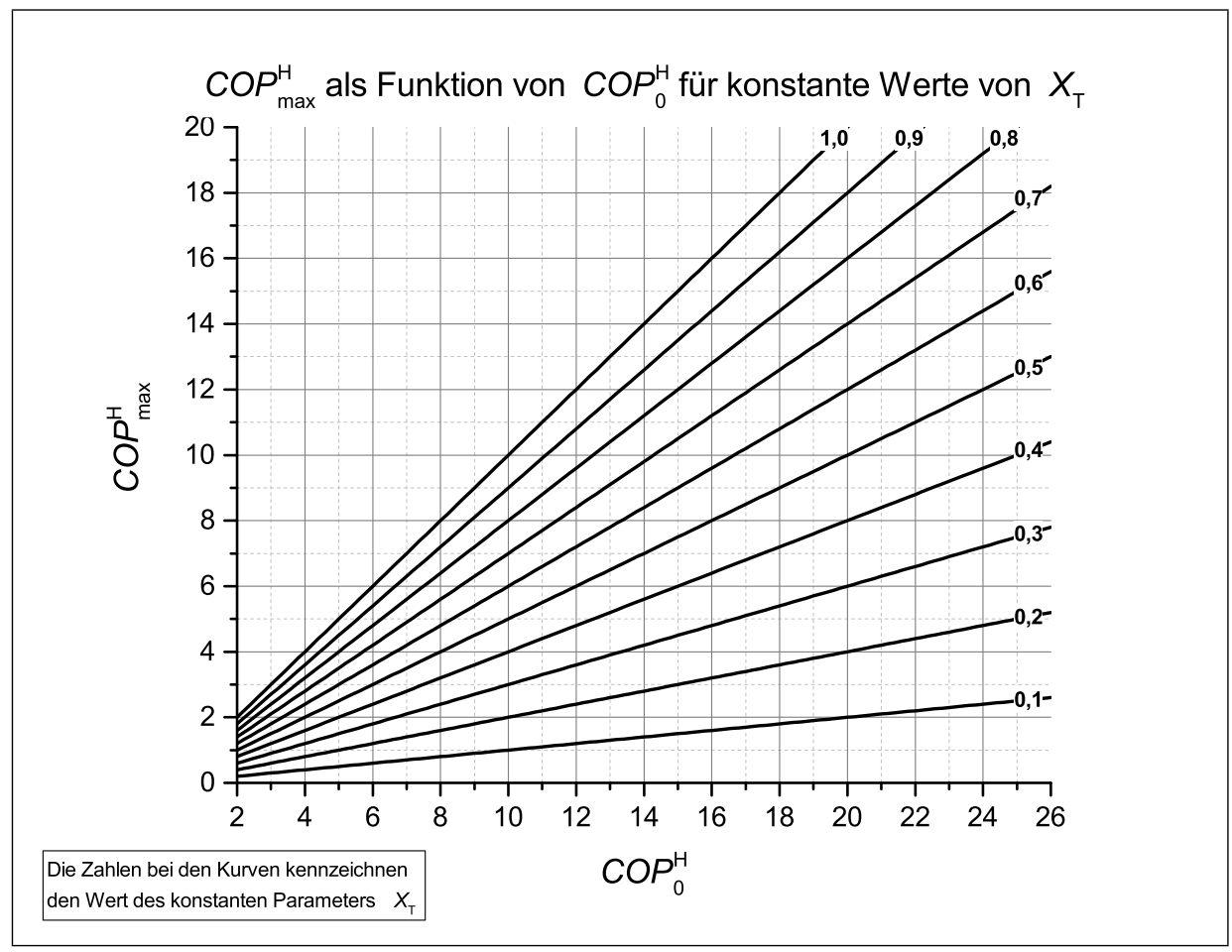

Abbildung 3.10: $C O P_{\max }^{\mathrm{H}}$ als Funktion von $C O P_{0}^{\mathrm{H}}$ im Intervall $2 \leq C O P_{0}^{\mathrm{H}} \leq 26$ für konstante Werte von $X_{\mathrm{T}}$ 


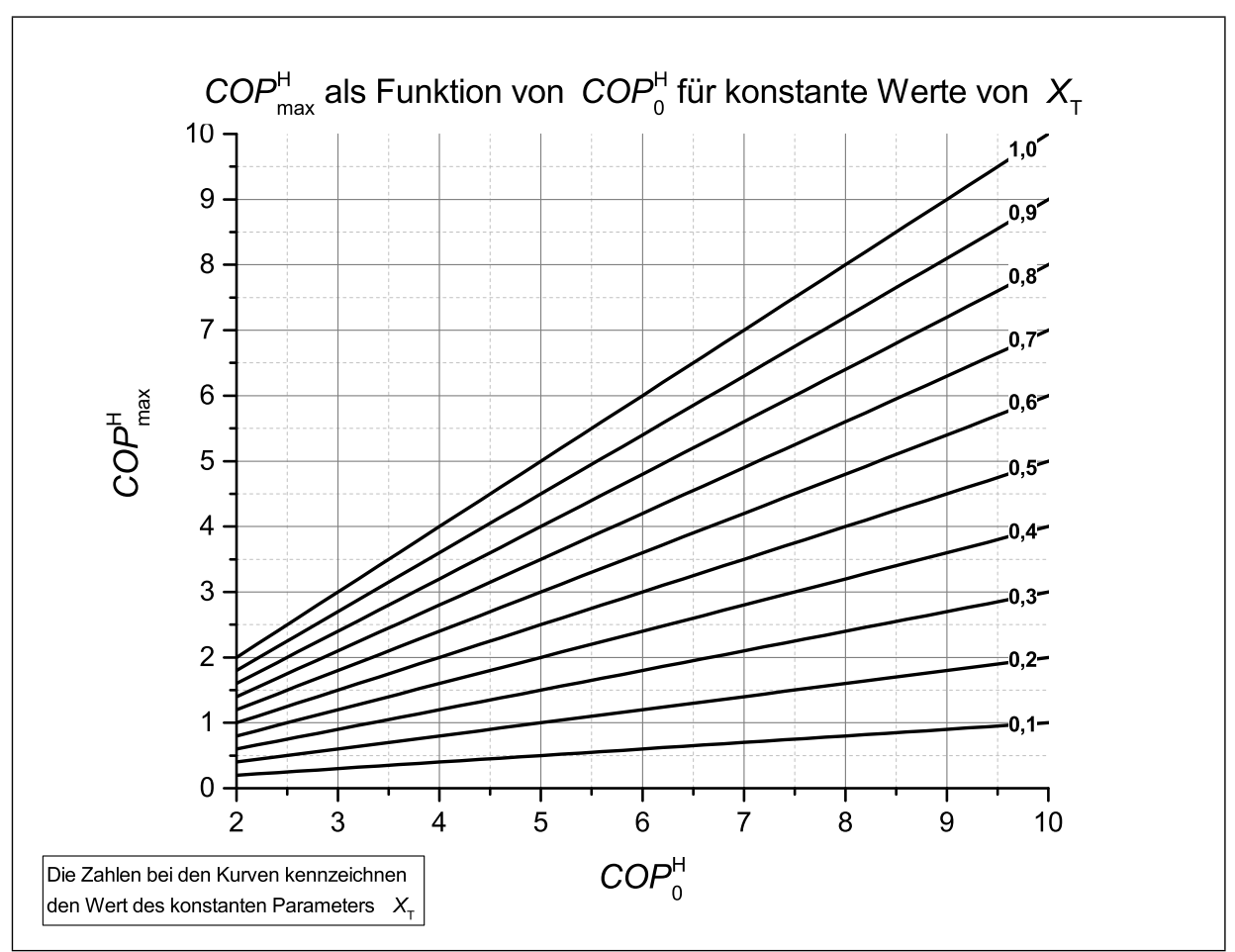

Abbildung 3.11: $C O P_{\max }^{\mathrm{H}}$ als Funktion von $C O P_{0}^{\mathrm{H}}$ im Intervall $2 \leq C O P_{0}^{\mathrm{H}} \leq 10$ für konstante Werte von $X_{\mathrm{T}}$

\section{Hinweise zu den Diagrammen zur Ermittlung von $C O P_{\max }^{\mathrm{H}}$}

- Auf der Abszisse in Abbildung 3.10 und 3.11 ist $C O P_{0}^{\mathrm{H}}$ aufgetragen. Als konstanter Parameter wurde $X_{\mathrm{T}}$ gewählt.

- Der Ableseweg wird im Folgenden am Beispiel von $C O P_{0}^{\mathrm{H}}$ und $X_{\mathrm{T}}=$ konstant beschrieben: Es wird eine Vertikale durch $C O P_{0}^{\mathrm{H}}$ gelegt und mit der korrespondierenden Kurve für $X_{\mathrm{T}}=$ konstant geschnitten. Für den ermittelten Schnittpunkt kann $C O P_{\max }^{\mathrm{H}}$ abgelesen werden.

- Für $C O P_{0}^{\mathrm{H}}=6$ und $X_{\mathrm{T}}=0,5$ folgt beispielsweise $C O P_{\max }^{\mathrm{H}}=3$. 
Zusammenfassung des Ablaufs zur Bestimmung von $\boldsymbol{C O P}_{\max }^{\mathrm{H}}$

Zur Bestimmung von $C O P_{\max }^{\mathrm{H}}$ werden die folgenden Schritte durchlaufen:

1. Ermittlung von $C O P_{0}^{\mathrm{H}}$

- Berechnung:

$$
C O P_{0}^{\mathrm{H}}=\frac{T_{\mathrm{S}}}{T_{\mathrm{S}}-T_{\mathrm{Q}}}
$$

- Abbildung 3.6 und 3.7 oder 3.8.

2. Ermittlung von $\widehat{\Delta T}$

- Berechnung:

$$
\begin{gathered}
\widehat{\Delta T}=\frac{\Delta T_{\mathrm{WT}}}{\Delta T_{\mathrm{SQ}}} \\
\Delta T_{\mathrm{SQ}}=T_{\mathrm{S}}-T_{\mathrm{Q}}
\end{gathered}
$$

- Abbildung 3.4 und 3.5.

3. Ermittlung von $X_{\mathrm{T}}$

- Berechnung:

$$
X_{\mathrm{T}}=1+\frac{\widehat{\Delta T}}{C O P_{0}^{\mathrm{H}}}\left(\frac{1-2 C O P_{0}^{\mathrm{H}}}{1+2 \widehat{\Delta T}}\right)
$$

- Abbildung 3.9.

4. Ermittlung von $C O P_{\max }^{\mathrm{H}}$

- Berechnung:

$$
C O P_{\max }^{\mathrm{H}}=X_{\mathrm{T}} C O P_{0}^{\mathrm{H}}
$$

- Abbildung 3.10 und 3.11. 


\subsubsection{Alternativer Ansatz zur Ermittlung von $C O P_{\max }^{\mathrm{H}}$}

Das in Kapitel 3.3.1 vorgestellte Konzept zur Ermittlung des maximalen COPs im Heizbetrieb hat, bedingt durch die Natur der dem Schema zugrunde liegenden Gleichung (vgl. $(3.2))$ :

$$
C O P_{\max }^{\mathrm{H}}=\frac{T_{\mathrm{S}}+\Delta T_{\mathrm{WT}}}{T_{\mathrm{S}}+2 \Delta T_{\mathrm{WT}}-T_{\mathrm{Q}}}
$$

welche es nicht erlaubt $C O P_{\max }^{\mathrm{H}}$ als Funktion von nur zwei Veränderlichen darzustellen, einige Nachteile:

1. Der Zusammenhang nach Gleichung (3.2) ist eine Funktion von drei Veränderlichen und kann daher nicht in Form eines einfachen Diagramms dargestellt werden. Für die direkte Ermittlung von $C O P_{\max }^{\mathrm{H}}$ unter Verwendung des vorgestellten Schemas resultiert daraus, neben einem erhöhten Aufwand, kein Nachteil. Die Beantwortung der inversen Fragestellung, z.B. wie hoch $\vartheta_{\mathrm{S}}$ maximal sein darf, damit bei gegebenem $\vartheta_{\mathrm{Q}}$ und $\Delta T_{\mathrm{WT}}$ ein fester Wert von $C O P_{\max }^{\mathrm{H}}$ nicht unterschritten wird, ist jedoch nicht mehr einfach möglich.

2. Das vorgestellte Schema zur Ermittlung von $C O P_{\max }^{\mathrm{H}}$ ist verhältnismäßig umfangreich und erweckt, mit Vorgriff auf Kapitel 6.1, den Eindruck, dass aus einer unkompliziert anmutenden Gleichung (vgl. (3.2)) ein kompliziertes Formelwerk abgeleitet wurde.

Zur Überwindung dieser prinzipbedingten Nachteile existiert ein einfacher Ansatz. Es wird zu Beginn $\Delta T_{\mathrm{WT}}$ ein konstanter Wert zugewiesen. Im Anschluss kann $C O P_{\max }^{\mathrm{H}}$ beim gewählten $\Delta T_{\mathrm{WT}}$ beispielsweise als Funktion von $\vartheta_{\mathrm{Q}}$ für konstante Werte von $\vartheta_{\mathrm{S}}$ dargestellt werden. Ein derartiges Diagramm bei $\Delta T_{\mathrm{WT}}=5{ }^{\circ} \mathrm{C}$ zeigt Abbildung 3.12: 


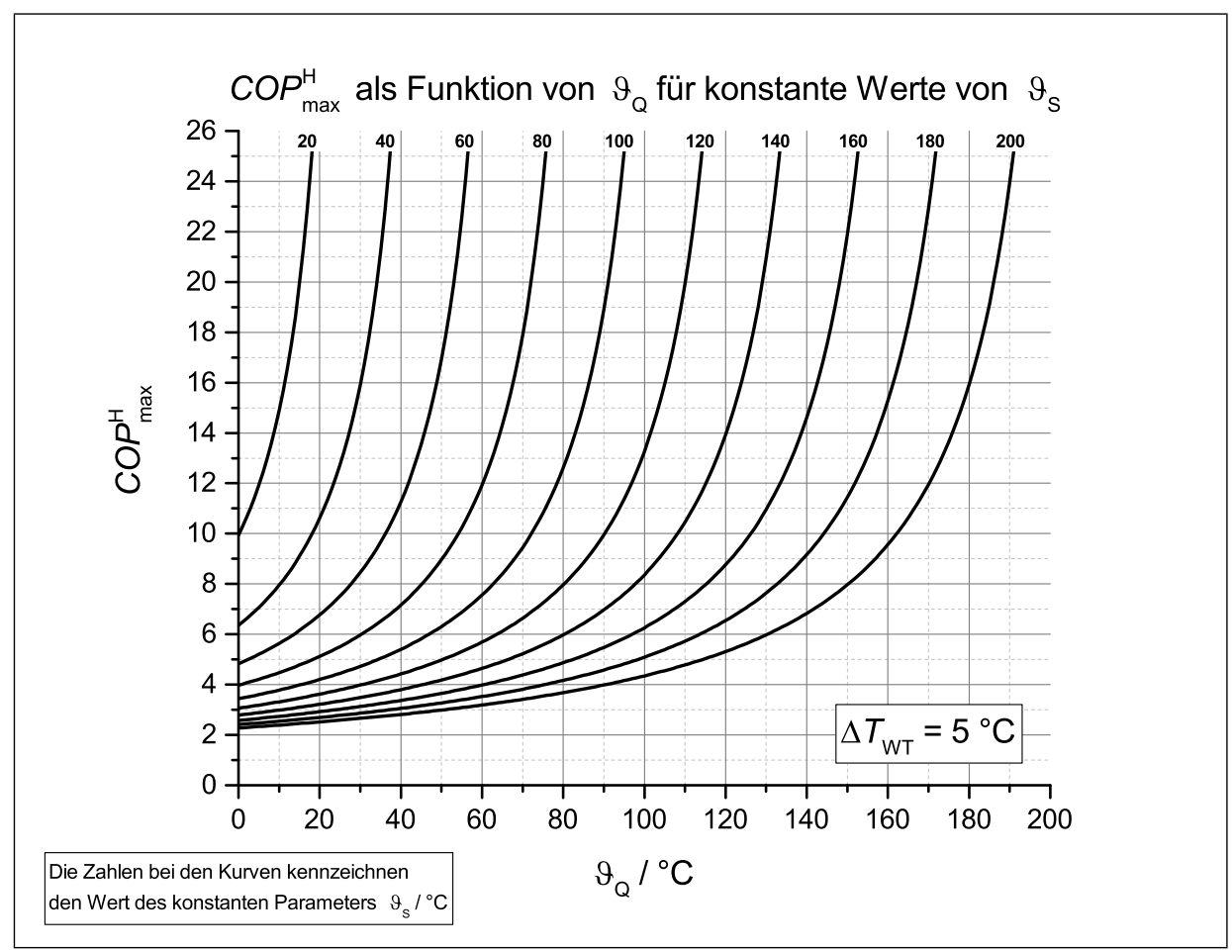

Abbildung 3.12: $C O P_{\max }^{\mathrm{H}}$ als Funktion von $\vartheta_{\mathrm{Q}}$ für konstante Werte von $\vartheta_{\mathrm{S}}$ bei $\Delta T_{\mathrm{WT}}=5{ }^{\circ} \mathrm{C}$

Dieses Prozedere für verschiedene Werte von $\Delta T_{\mathrm{WT}}$ wiederholt, ergibt einen Satz an Diagrammen, wodurch die Ermittlung von $C O P_{\max }^{\mathrm{H}}$ bei verschiedenen Werte von $\Delta T_{\mathrm{WT}}$ ermöglicht wird. Ob dies das Vorgehen der Wahl ist, wird in Kapitel 6.3 erneut aufgegriffen.

Es existiert jedoch zumindest ein weiterer Ansatz, der auf eine Vielzahl von Diagrammen verzichtet und für ein Verfahren, das bedingt durch Ablesefehler zumeist nie ganz exakt sein wird, als ausreichend genau einzustufen ist.

Aus der Beobachtung kann abgeleitet werden, dass $C O P_{\max }^{\mathrm{H}}$ für bestimmte Konstellationen von $\vartheta_{\mathrm{S}}$ und $\Delta T_{\mathrm{WT}}$ bei gleichem $\vartheta_{\mathrm{Q}}$ annähernd identisch ist. Wird zum Beispiel gesetzt:

$$
\begin{array}{ll}
\vartheta_{\mathrm{S}}^{1}=40{ }^{\circ} \mathrm{C} & \Delta T_{\mathrm{WT}}^{1}=5{ }^{\circ} \mathrm{C} \\
\vartheta_{\mathrm{S}}^{2}=20{ }^{\circ} \mathrm{C} & \Delta T_{\mathrm{WT}}^{2}=15^{\circ} \mathrm{C}
\end{array}
$$

dann gilt (ohne Beweis):

$$
C O P_{\max }^{\mathrm{H}}\left(\vartheta_{\mathrm{S}}^{1}, \vartheta_{\mathrm{Q}}, \Delta T_{\mathrm{WT}}^{1}\right) \sim C O P_{\max }^{\mathrm{H}}\left(\vartheta_{\mathrm{S}}^{2}, \vartheta_{\mathrm{Q}}, \Delta T_{\mathrm{WT}}^{2}\right)
$$

sowie:

$$
\frac{\left|C O P_{\max }^{\mathrm{H}}\left(\vartheta_{\mathrm{S}}^{1}, \vartheta_{\mathrm{Q}}, \Delta T_{\mathrm{WT}}^{1}\right)-C O P_{\max }^{\mathrm{H}}\left(\vartheta_{\mathrm{S}}^{2}, \vartheta_{\mathrm{Q}}, \Delta T_{\mathrm{WT}}^{2}\right)\right|}{C O P_{\max }^{\mathrm{H}}\left(\vartheta_{\mathrm{S}}^{2}, \vartheta_{\mathrm{Q}}, \Delta T_{\mathrm{WT}}^{2}\right)}=\text { konstant }
$$

Die qualitative Richtigkeit dieser Feststellungen kann graphisch überprüft werden. Abbildung 3.13 zeigt $C O P_{\max }^{\mathrm{H}}$ für die Parameterkonstellation aus Gleichung (3.13): 


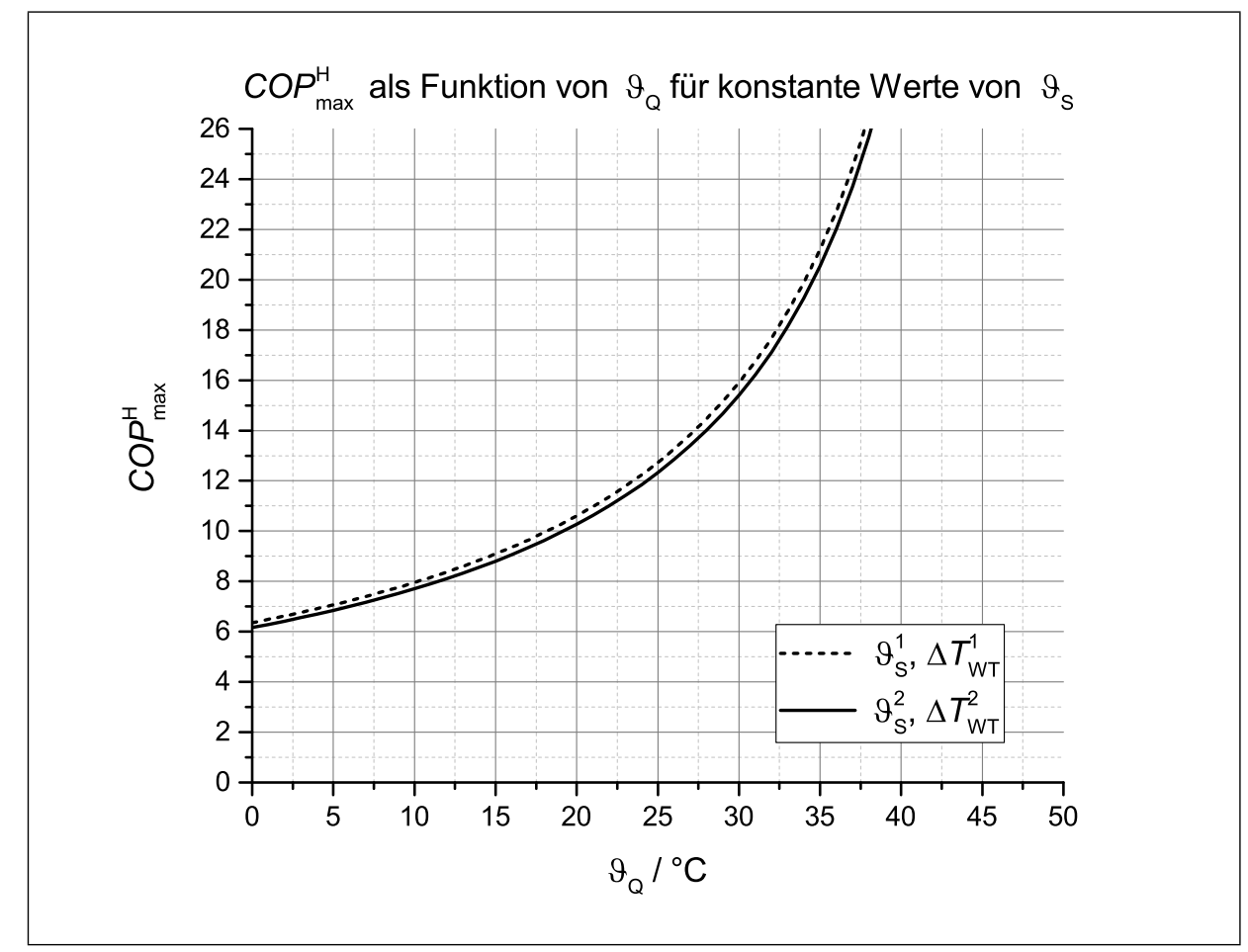

Abbildung 3.13: $C O P_{\max }^{\mathrm{H}}\left(\vartheta_{\mathrm{S}}^{1}, \vartheta_{\mathrm{Q}}, \Delta T_{\mathrm{WT}}^{1}\right)$ und $C O P_{\max }^{\mathrm{H}}\left(\vartheta_{\mathrm{S}}^{2}, \vartheta_{\mathrm{Q}}, \Delta T_{\mathrm{WT}}^{2}\right)$

Um die Erkenntnis, dass $C O P_{\max }^{\mathrm{H}}$ für bestimmte Konstellationen von $\vartheta_{\mathrm{S}}$ und $\Delta T_{\mathrm{WT}}$ bei gleichem $\vartheta_{\mathrm{Q}}$ annähernd identisch ist, nutzen zu können, muss eine Gesetzmäßigkeit für die veränderlichen Größen, in nachfolgender Gleichung mit dem hochgestellten Index 1 und 2 gekennzeichnet, die:

$$
\begin{gathered}
C O P_{\max }^{\mathrm{H}}\left(\vartheta_{\mathrm{S}}^{1}, \vartheta_{\mathrm{Q}}, \Delta T_{\mathrm{WT}}^{1}\right) \sim C O P_{\max }^{\mathrm{H}}\left(\vartheta_{\mathrm{S}}^{2}, \vartheta_{\mathrm{Q}}, \Delta T_{\mathrm{WT}}^{2}\right) \\
\frac{\left|C O P_{\max }^{\mathrm{H}}\left(\vartheta_{\mathrm{S}}^{1}, \vartheta_{\mathrm{Q}}, \Delta T_{\mathrm{WT}}^{1}\right)-C O P_{\max }^{\mathrm{H}}\left(\vartheta_{\mathrm{S}}^{2}, \vartheta_{\mathrm{Q}}, \Delta T_{\mathrm{WT}}^{2}\right)\right|}{C O P_{\max }^{\mathrm{H}}\left(\vartheta_{\mathrm{S}}^{1}, \vartheta_{\mathrm{Q}}, \Delta T_{\mathrm{WT}}^{1}\right)}=\text { konstant } \\
\frac{\left|C O P_{\max }^{\mathrm{H}}\left(\vartheta_{\mathrm{S}}^{1}, \vartheta_{\mathrm{Q}}, \Delta T_{\mathrm{WT}}^{1}\right)-C O P_{\max }^{\mathrm{H}}\left(\vartheta_{\mathrm{S}}^{2}, \vartheta_{\mathrm{Q}}, \Delta T_{\mathrm{WT}}^{2}\right)\right|}{C O P_{\max }^{\mathrm{H}}\left(\vartheta_{\mathrm{S}}^{2}, \vartheta_{\mathrm{Q}}, \Delta T_{\mathrm{WT}}^{2}\right)}=\text { konstant }
\end{gathered}
$$

erfüllt, gefunden werden.

Zur Vereinfachung der Schreibweise werden das vorgegebene Tupel $\vartheta_{1}^{*}$ und das abgeleitete Tupel $\vartheta_{2}^{*}$ mit:

$$
\begin{aligned}
& \vartheta_{1}^{*}=\left(\vartheta_{\mathrm{S}}^{1}, \vartheta_{\mathrm{Q}}, \Delta T_{\mathrm{WT}}^{1}\right) \\
& \vartheta_{2}^{*}=\left(\vartheta_{\mathrm{S}}^{2}, \vartheta_{\mathrm{Q}}, \Delta T_{\mathrm{WT}}^{2}\right)
\end{aligned}
$$

eingeführt. 
Diese Einführung erlaubt Gleichung (3.16) in:

$$
\begin{gathered}
C O P_{\text {max }}^{\mathrm{H}}\left(\vartheta_{1}^{*}\right) \sim C O P_{\text {max }}^{\mathrm{H}}\left(\vartheta_{2}^{*}\right) \\
\frac{\left|C O P_{\text {max }}^{\mathrm{H}}\left(\vartheta_{1}^{*}\right)-C O P_{\max }^{\mathrm{H}}\left(\vartheta_{2}^{*}\right)\right|}{C O P_{\max }^{\mathrm{H}}\left(\vartheta_{1}^{*}\right)}=\text { konstant } \\
\frac{\left|C O P_{\max }^{\mathrm{H}}\left(\vartheta_{1}^{*}\right)-C O P_{\text {max }}^{\mathrm{H}}\left(\vartheta_{2}^{*}\right)\right|}{C O P_{\text {max }}^{\mathrm{H}}\left(\vartheta_{2}^{*}\right)}=\text { konstant }
\end{gathered}
$$

zu überführen.

Hinweis: Für alle in diesem Kapitel vorgestellten Ansätze zur Berechnung jener Tupel $\vartheta_{2}^{*}$ aus $\vartheta_{1}^{*}$, die Gleichung (3.18) erfüllen, gilt, dass diese ohne Beweis vorgestellt werden. Der Zusatz "ohne Beweis" entfällt daher. In Kapitel 6.1 wird auf die vorgestellten Ansätze und eventuell in diesem Zusammenhang notwendige Folgearbeiten eingegangen.

Es gibt mehrere Ansätze die Gleichung (3.18) erfüllen, jedoch sind nicht alle zielführend. So gilt beispielsweise bei Vorgabe von $\Delta T_{\mathrm{WT}}^{1}$, unter Einhaltung von:

$$
\begin{array}{r}
\vartheta_{\mathrm{S}}^{1}=8 \Delta T_{\mathrm{WT}}^{1} \\
\vartheta_{\mathrm{S}}^{2}=4 \Delta T_{\mathrm{WT}}^{1} \\
\Delta T_{\mathrm{WT}}^{2}=3 \Delta T_{\mathrm{WT}}^{1}
\end{array}
$$

dass Gleichung (3.18) erfüllt ist. Als nachteilig erweist sich jedoch die Tatsache, dass multiplikative Ansätze für die gegebene Aufgabenstellung eher ungünstig sind, da die Parameter zumeist eher durch additive Konstanten sinnvoll voneinander zu unterscheiden sind. Wenn eine wie auch immer geartete Kurve, hier bei Vernachlässigung von $\Delta T_{\mathrm{WT}}$, für $\vartheta_{\mathrm{S}}^{1}$ gültig ist, dann ist im vorliegenden Fall der Erkenntnisgewinn höher, wenn diese Kurve ebenfalls für $\vartheta_{\mathrm{S}}^{2}=\vartheta_{\mathrm{S}}^{1}+A$ und anstelle von $\vartheta_{\mathrm{S}}^{2}=\vartheta_{\mathrm{S}}^{1} \cdot A$ ihre Gültigkeit besitzt.

Gesucht wird daher ein Ansatz der Form:

$$
\begin{aligned}
\vartheta_{\mathrm{S}}^{2} & =\vartheta_{\mathrm{S}}^{1}+A \\
\Delta T_{\mathrm{WT}}^{2} & =\Delta T_{\mathrm{WT}}^{1}+B \\
\mathrm{~B} & =f(A)
\end{aligned}
$$

welcher bei Vorgabe von $\vartheta_{\mathrm{S}}^{1}$ und $\Delta T_{\mathrm{WT}}^{1}$ Gleichung (3.18) für alle $A, B \in \mathbb{R}$ erfüllt. 
Für das Verständnis der nachfolgenden Ausführungen, sei an dieser Stelle das Ziel noch einmal explizit hervorgehoben:

Es ist ein Zusammenhang zu ermitteln, der bei Vorgabe von einem Tupel $\vartheta_{1}^{*}$ die Berechnung von unendlich vielen Tupel $\vartheta_{2}^{*}$ ermöglicht, welche nur noch von $A$ abhängen sollen.

Für alle ermittelten Tupel $\vartheta_{2}^{*}$ soll gelten, dass sie zusammen mit $\vartheta_{1}^{*}$ Gleichung (3.18) erfüllen.

Gefunden wurde der folgende Zusammenhang:

$$
\begin{aligned}
\vartheta_{\mathrm{S}}^{2} & =\vartheta_{\mathrm{S}}^{1}+A \\
\Delta T_{\mathrm{WT}}^{2} & =\Delta T_{\mathrm{WT}}^{1}+B \\
B & =-\frac{A}{2}
\end{aligned}
$$

Schema zur Bestimmung von $\vartheta_{2}^{*}$ aus $\vartheta_{1}^{*}$ unter Erfüllung der Bedingungen aus Gleichung (3.18)

Das Resultat aus Gleichung (3.21) kann wie folgt beschrieben werden:

Bei Vorgabe von $\vartheta_{1}^{*}$ gilt, dass Gleichung (3.18) erfüllt ist, wenn die Komponenten von $\vartheta_{2}^{*}$ unter Verwendung von:

$$
\begin{gathered}
\vartheta_{\mathrm{S}}^{2}=\vartheta_{\mathrm{S}}^{1}+A \\
\Delta T_{\mathrm{WT}}^{2}=\Delta T_{\mathrm{WT}}^{1}-\frac{A}{2}
\end{gathered}
$$

bestimmt werden. Aus der Vorgabe eines Tupel $\vartheta_{1}^{*}$ folgen daher unendlich viele Tupel $\vartheta_{2}^{*}$ die nur noch von $A \in \mathbb{R}$ abhängen. Das beschreibt genau jene Aufgabe, die es zu erfüllen galt.

In weiterer Folge gilt es, das Intervall von $A$ soweit einzuschränken, dass der Einsatz von Gleichung (3.22) nur noch technisch sinnvolle Lösungen hervorbringt. Berücksichtigt wurde:

- Im Anwendungsfeld der Wärmepumpe sind nur Vorgabewerte von $\Delta T_{\mathrm{WT}}^{1} \geq 0{ }^{\circ} \mathrm{C}$ sinnvoll. Es muss daher die Entscheidung getroffen werden, ob der Minimalwert oder der Maximalwert von $\Delta T_{\mathrm{WT}}^{1}$ zur Eingrenzung von $A$ angegeben werden soll. In der vorliegenden Arbeit ist entschieden worden:

$$
\Delta T_{\mathrm{WT}}^{1}=\left.\Delta T_{\mathrm{WT}}\right|_{\max }
$$

zu setzten. Die Begründung dieser Entscheidung wird erst im späterem Verlauf klar. An dieser Stelle habe sie daher den Charakter einer Definition. 
- Nicht nur $\Delta T_{\mathrm{WT}}^{1}$ sondern auch $\Delta T_{\mathrm{WT}}^{2}$ ist in technischen Anwendungen stets einer Begrenzung unterworfen. Nachdem es sich bei beiden Temperaturdifferenzen um charakteristische Temperaturdifferenzen im Sinne von Kapitel 3.2 handelt, wird gesetzt:

$$
0{ }^{\circ} \mathrm{C} \leq \Delta T_{\mathrm{WT}}^{2} \leq\left.\Delta T_{\mathrm{WT}}\right|_{\max }
$$

- Aus Gleichung (3.23) und (3.24) folgt für das Intervall von $A$ :

1. Unter Grenze:

$$
A \geq 0{ }^{\circ} \mathrm{C} \longrightarrow A_{\min }=0{ }^{\circ} \mathrm{C}
$$

2. Obere Grenze:

$$
\begin{gathered}
\Delta T_{\mathrm{WT}}^{1}-\frac{A}{2} \geq 0{ }^{\circ} \mathrm{C} \longrightarrow A_{\mathrm{max}}=2 \cdot \Delta T_{\mathrm{WT}}^{1} \\
\longrightarrow A_{\max }=\left.2 \cdot \Delta T_{\mathrm{WT}}\right|_{\max }
\end{gathered}
$$

3. Gesamtes Intervall:

$$
0{ }^{\circ} \mathrm{C} \leq A \leq\left. 2 \cdot \Delta T_{\mathrm{WT}}\right|_{\max }
$$

Das Berechnungsschema inklusive der ermittelten Intervalle lautet daher:

$$
\begin{gathered}
\vartheta_{\mathrm{S}}^{2}=\vartheta_{\mathrm{S}}^{1}+A \\
\Delta T_{\mathrm{WT}}^{2}=\Delta T_{\mathrm{WT}}^{1}-\frac{A}{2} \\
\Delta T_{\mathrm{WT}}^{1}=\left.\Delta T_{\mathrm{WT}}\right|_{\max } \\
0{ }^{\circ} \mathrm{C} \leq A \leq\left. 2 \cdot \Delta T_{\mathrm{WT}}\right|_{\max }
\end{gathered}
$$

Aus Gleichung (3.28) folgt für den Wertebereich der Komponenten von $\vartheta_{2}^{*}$ unter Vorgabe der Komponenten von $\vartheta_{1}^{*}$ :

$$
\begin{gathered}
0{ }^{\circ} \mathrm{C} \leq \Delta T_{\mathrm{WT}}^{2} \leq\left.\Delta T_{\mathrm{WT}}\right|_{\max } \\
\vartheta_{\mathrm{S}}^{1} \leq \vartheta_{\mathrm{S}}^{2} \leq \vartheta_{\mathrm{S}}^{1}+\left.2 \cdot \Delta T_{\mathrm{WT}}\right|_{\max }
\end{gathered}
$$


Zusammenfassend lässt sich daher feststellen:

- Wenn die Komponenten von $\vartheta_{1}^{*}$ vorgegeben und die Komponenten von $\vartheta_{2}^{*}$ nach Gleichung (3.28) berechnet werden, dann gilt:

- Der Wertebereich der Komponenten von $\vartheta_{2}^{*}$ ist nach Gleichung (3.29) gegeben.

- $C O P_{\max }^{\mathrm{H}}\left(\vartheta_{1}^{*}\right)$ entspricht ungefähr $C O P_{\max }^{\mathrm{H}}\left(\vartheta_{2}^{*}\right)$.

- Der relative Fehler ist für feste Werte der Komponenten von $\vartheta_{1}^{*}$ und $A$ konstant und hängt nicht von $\vartheta_{\mathrm{Q}}$ ab.

Beispiel:

- Gegeben sei $\vartheta_{\mathrm{S}}^{1}=120^{\circ} \mathrm{C}$ und $\left.\Delta T_{\mathrm{WT}}\right|_{\max }=20^{\circ} \mathrm{C}$.

- Dann gilt, dass $C O P_{\max }^{\mathrm{H}}\left(\vartheta_{\mathrm{S}}, \vartheta_{\mathrm{Q}}, \Delta T_{\mathrm{WT}}\right)$ für die folgenden Eingabeparameter nahezu ident ist:

$$
\begin{array}{ccc}
\text { Gegeben }: & \vartheta_{\mathrm{S}}^{1}=120{ }^{\circ} \mathrm{C} & \Delta T_{\mathrm{WT}}^{1}=20{ }^{\circ} \mathrm{C} \\
A=0{ }^{\circ} \mathrm{C} \rightarrow & \vartheta_{\mathrm{S}}^{2}=120{ }^{\circ} \mathrm{C} & \Delta T_{\mathrm{WT}}^{2}=20{ }^{\circ} \mathrm{C} \\
A=10{ }^{\circ} \mathrm{C} \rightarrow & \vartheta_{\mathrm{S}}^{2}=130{ }^{\circ} \mathrm{C} & \Delta T_{\mathrm{WT}}^{2}=15^{\circ} \mathrm{C} \\
A=20{ }^{\circ} \mathrm{C} \rightarrow & \vartheta_{\mathrm{S}}^{2}=140{ }^{\circ} \mathrm{C} & \Delta T_{\mathrm{WT}}^{2}=10^{\circ} \mathrm{C} \\
A=30{ }^{\circ} \mathrm{C} \rightarrow & \vartheta_{\mathrm{S}}^{2}=150{ }^{\circ} \mathrm{C} & \Delta T_{\mathrm{WT}}^{2}=5{ }^{\circ} \mathrm{C} \\
A=40{ }^{\circ} \mathrm{C} \rightarrow & \vartheta_{\mathrm{S}}^{2}=160{ }^{\circ} \mathrm{C} & \Delta T_{\mathrm{WT}}^{2}=0{ }^{\circ} \mathrm{C}
\end{array}
$$

- Bei Auswertung der Berechnungsergebnisse lässt sich weiteres feststellen:

- Der relative Fehler, hier gegeben durch den Betrag der Differenz von $C O P_{\max }^{\mathrm{H}}\left(\vartheta_{1}^{*}\right)$ und $C O P_{\max }^{\mathrm{H}}\left(\vartheta_{2}^{*}\right)$ normiert mit dem Maximum aus $C O P_{\max }^{\mathrm{H}}\left(\vartheta_{1}^{*}\right)$ und $C O P_{\max }^{\mathrm{H}}\left(\vartheta_{2}^{*}\right)$, ist für ein gegebenes $A$ konstant. Der auf diese Art und Weise gewonnene relative Fehler ist nicht von $\vartheta_{\mathrm{Q}}$ abhängig und beträgt bei:

$$
\begin{array}{ccc}
\text { Gegeben : } & \vartheta_{\mathrm{S}}^{1}=120{ }^{\circ} \mathrm{C} & \Delta T_{\mathrm{WT}}^{1}=20{ }^{\circ} \mathrm{C} \\
A=40{ }^{\circ} \mathrm{C} \rightarrow & \vartheta_{\mathrm{S}}^{2}=160{ }^{\circ} \mathrm{C} & \Delta T_{\mathrm{WT}}^{2}=0{ }^{\circ} \mathrm{C}
\end{array}
$$

beispielsweise 0,046173381 .

- Der relative Fehler ist umso größer, je größer $A$ und je kleiner $\vartheta_{\mathrm{S}}^{1}$ ist. Aus:

$$
\begin{array}{cll}
\text { Gegeben : } & \vartheta_{\mathrm{S}}^{1}=100{ }^{\circ} \mathrm{C} & \Delta T_{\mathrm{WT}}^{1}=20{ }^{\circ} \mathrm{C} \\
A=40{ }^{\circ} \mathrm{C} \rightarrow & \vartheta_{\mathrm{S}}^{2}=140{ }^{\circ} \mathrm{C} & \Delta T_{\mathrm{WT}}^{2}=0{ }^{\circ} \mathrm{C}
\end{array}
$$

resultiert zum Beispiel ein relativer Fehler von 0,048408568. 


\section{Einführung von $\boldsymbol{C O P}_{\max }^{\mathrm{H}}$}

Es wird der gemittelte maximale COP im Heizbetrieb eingeführt:

$$
\overline{C O P}_{\max }^{\mathrm{H}}\left(\vartheta_{1}^{*}\right):=\frac{C O P_{\max }^{\mathrm{H}}\left(\vartheta_{1}^{*}\right)+\left.C O P_{\max }^{\mathrm{H}}\left(\vartheta_{2}^{*}\right)\right|_{A=A_{\max }}}{2}
$$

Für ihn gelte:

- $\vartheta_{1}^{*}$ und $\left.\Delta T_{\mathrm{WT}}\right|_{\max }$ sind vorzugeben.

- $\vartheta_{2}^{*}$ wird unter Verwendung von Gleichung (3.28) bei $A=A_{\max }$ berechnet.

Der wesentliche Vorteil von $\overline{C O P}_{\max }^{\mathrm{H}}$ ist, dass er in Diagrammform abgebildet werden kann und jede Kurve $\vartheta_{1}^{*}$ mit hinreichender Genauigkeit für alle Tupel von $\vartheta_{2}^{*}$ aus $\vartheta_{1}^{*}$ gültig ist. Ein Beispiel folgt im Unterkapitel zur Ableitung der Diagramme für $\overline{C O P_{\max }^{\mathrm{H}}}$.

Für die Differenz:

$$
\Delta\left(C O P_{\max }^{\mathrm{H}}\left(\vartheta_{1}^{*}\right)\right)=\left|C O P_{\max }^{\mathrm{H}}\left(\vartheta_{1}^{*}\right)-C O P_{\max }^{\mathrm{H}}\left(\vartheta_{2}^{*}\right)\right|
$$

gilt, dass diese ihren Maximalwert bei $\mathrm{A}=\mathrm{A}_{\max }$ aufweist.

Da $\overline{C O P}_{\max }^{\mathrm{H}}$ per Definition bei $A=A_{\max }$ ermittelt wird, folgt für den maximalen relativen Fehler:

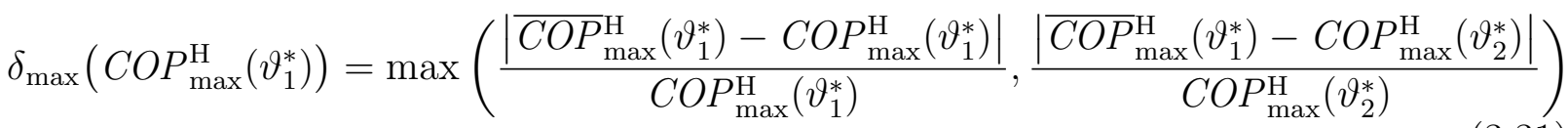

Für den maximalen relative Fehler gilt (ohne Beweis):

$$
\delta_{\max }\left(C O P_{\max }^{\mathrm{H}}\left(\vartheta_{1}^{*}\right)\right)=\frac{\mid \overline{C O P_{\max }^{\mathrm{H}}\left(\vartheta_{1}^{*}\right)-C O P_{\max }^{\mathrm{H}}\left(\vartheta_{1}^{*}\right) \mid}}{C O P_{\max }^{\mathrm{H}}\left(\vartheta_{1}^{*}\right)}=\text { konstant }
$$

Hinweis: Die hier verwendete Definition des relativen Fehlers erlaubt festzustellen, wie weit $C O P_{\max }^{\mathrm{H}}\left(\vartheta^{*}\right)$ von seinem wahren Wert abweicht, wenn $C O P_{\max }^{\mathrm{H}}\left(\vartheta^{*}\right)$ aus einem Diagramm

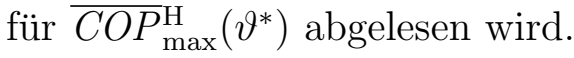




\section{Ableitung von Diagrammen für $\boldsymbol{C O P}_{\max }^{\mathrm{H}}$}

Zur Ableitung von Diagrammen für $\overline{C O P}_{\max }^{\mathrm{H}}$ wird der Zusammenhang nach Gleichung (3.30) herangezogen und unter Verwendung der folgenden Randbedingungen visualisiert:

$$
\begin{aligned}
\left.\Delta T_{\mathrm{WT}}\right|_{\max } & =20^{\circ} \mathrm{C} \\
\vartheta_{\mathrm{S}}^{1} & =\left\{0{ }^{\circ} \mathrm{C}, 20{ }^{\circ} \mathrm{C}, 40{ }^{\circ} \mathrm{C}, \ldots, 160{ }^{\circ} \mathrm{C}, 180{ }^{\circ} \mathrm{C}\right\}
\end{aligned}
$$

Das Resultat zeigt Abbildung 3.14:

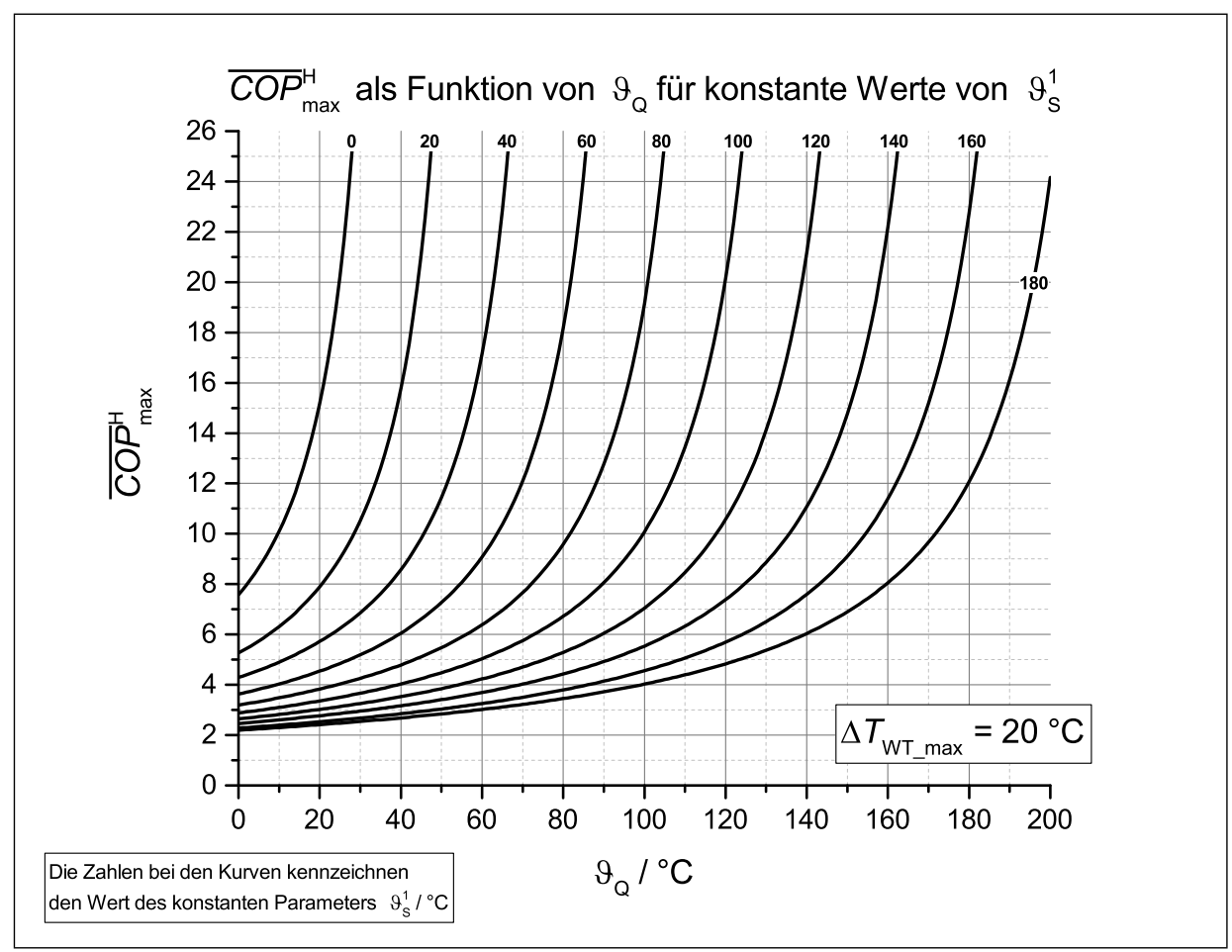

Abbildung 3.14: $\overline{C O P}_{\max }^{\mathrm{H}}$ als Funktion von $\vartheta_{\mathrm{Q}}$ für konstante Werte von $\vartheta_{\mathrm{S}}^{1}$

Grundsätzlich gilt, dass Abbildung 3.14 für alle $A \leq A_{\max }$ verwendet werden kann. Im Rahmen der Anwendung werden jedoch nur diskrete aussagekräftige Werte von $A$ zur Verwendung kommen. Tabelle 3.1 bietet eine Hilfe für den Ableseprozess. Es ist zu beachten, dass alle Temperaturwerte in Tabelle 3.1 durch ${ }^{\circ} \mathrm{C}$ dividiert wurden.

An den Ausführungen ist ebenfalls zu erkennen, warum $\Delta T_{\mathrm{WT}}^{1}=\left.\Delta T_{\mathrm{WT}}\right|_{\max }$ gesetzt wurde. Dadurch wird gewährleistet, dass das Berechnungsschema für die Visualisierung günstige Parameterkonstellationen ergibt. 
Tabelle 3.1: Ablesehilfe für Abbildung 3.14

\begin{tabular}{rrrrrrrr}
\hline & $A=0$ & $A=10$ & $A=20$ & \multicolumn{1}{c}{$A=30$} & \multicolumn{1}{c}{$A=40$} \\
\hline$\vartheta_{\mathrm{S}}^{1} \mid \Delta T_{\mathrm{WT}}^{1}$ & $\vartheta_{\mathrm{S}}^{2} \mid \Delta T_{\mathrm{WT}}^{2}$ & $\vartheta_{\mathrm{S}}^{2} \mid \Delta T_{\mathrm{WT}}^{2}$ & $\vartheta_{\mathrm{S}}^{2} \mid \Delta T_{\mathrm{WT}}^{2}$ & $\vartheta_{\mathrm{S}}^{2} \mid \Delta T_{\mathrm{WT}}^{2}$ & $\vartheta_{\mathrm{S}}^{2} \mid \Delta T_{\mathrm{WT}}^{2}$ & $\delta_{\max }$ \\
\hline $180 \mid 20$ & $180 \mid 20$ & $190 \mid 15$ & $200 \mid 10$ & $210 \mid 5$ & $220 \mid 0$ & 0,021135 \\
$160 \mid 20$ & $160 \mid 20$ & $170 \mid 15$ & $180 \mid 10$ & $190 \mid 5$ & $200 \mid 0$ & 0,022068 \\
$140 \mid 20$ & $140 \mid 20$ & $150 \mid 15$ & $160 \mid 10$ & $170 \mid 5$ & $180 \mid 0$ & 0,023087 \\
$120 \mid 20$ & $120 \mid 20$ & $130 \mid 15$ & $140 \mid 10$ & $150 \mid 5$ & $160 \mid 0$ & 0,024204 \\
$100 \mid 20$ & $100 \mid 20$ & $110 \mid 15$ & $120 \mid 10$ & $130 \mid 5$ & $140 \mid 0$ & 0,025436 \\
$80 \mid 20$ & $80 \mid 20$ & $90 \mid 15$ & $100 \mid 10$ & $110 \mid 5$ & $120 \mid 0$ & 0,026799 \\
$60 \mid 20$ & $60 \mid 20$ & $70 \mid 15$ & $80 \mid 10$ & $90 \mid 5$ & $100 \mid 0$ & 0,028317 \\
$40 \mid 20$ & $40 \mid 20$ & $50 \mid 15$ & $60 \mid 10$ & $70 \mid 5$ & $80 \mid 0$ & 0,030017 \\
$20 \mid 20$ & $20 \mid 20$ & $30 \mid 15$ & $40 \mid 10$ & $50 \mid 5$ & $60 \mid 0$ & 0,031934 \\
$0 \mid 20$ & $0 \mid 20$ & $10 \mid 15$ & $20 \mid 10$ & $30 \mid 5$ & $40 \mid 0$ & 0,034112 \\
\hline
\end{tabular}

Hinweise zu den Diagrammen zur Ermittlung $\overline{C O P}_{\max }^{\mathrm{H}}$

Abbildung 3.14 und Tabelle 3.1 werden wie folgt verwendet:

- $\overline{C O P}_{\max }^{\mathrm{H}}$ ist für jede Zeile in Tabelle 3.1 annähernd konstant.

- $\delta_{\max }$ ist für jede Zeile in Tabelle 3.1 konstant.

- Abbildung 3.14 zeigt $\overline{C O P}{ }_{\max }^{\mathrm{H}}$ für die Kombinationen aus $\vartheta_{\mathrm{S}}^{1}$ und $\Delta T_{\mathrm{WT}}^{1}$, die in der 1. Spalte von Tabelle 3.1 stehen.

- Soll zum Beispiel $\overline{C O P_{\max }^{\mathrm{H}}}$ von $\vartheta_{\mathrm{S}}=110{ }^{\circ} \mathrm{C}, \vartheta_{\mathrm{Q}}=60^{\circ} \mathrm{C}$ und $\Delta T_{\mathrm{WT}}=5{ }^{\circ} \mathrm{C}$ bestimmt werden, so kann aus Tabelle 3.1 ermittelt werden, dass $\vartheta_{\mathrm{S}}=110^{\circ} \mathrm{C}$ und $\Delta T_{\mathrm{WT}}=5{ }^{\circ} \mathrm{C}$ der Kombination $\vartheta_{\mathrm{S}}^{1}=80{ }^{\circ} \mathrm{C}$ und $\Delta T_{\mathrm{WT}}^{1}=20^{\circ} \mathrm{C}$ entspricht.

Wird daher gedanklich in Abbildung 3.14 eine Vertikale durch $\vartheta_{\mathrm{Q}}=60{ }^{\circ} \mathrm{C}$ gelegt und mit der Kurve für $\vartheta_{\mathrm{S}}^{1}=80{ }^{\circ} \mathrm{C}$ zum Schnitt gebracht, so folgt für $\overline{C O P_{\max }^{\mathrm{H}}}$, dass dieser ungefähr 6,4 beträgt. Auf dem Rechenweg lässt sich für diese Eingabekombination ein $C O P_{\max }^{\mathrm{H}}=6,4692$ ermitteln. 


\section{Hinweise zur Anwendung von $\overline{C O P_{\max }^{\mathrm{H}}}$ und $C O P_{\max }^{\mathrm{H}}$}

- Das Anwendungsfeld von $C O P_{\max }^{\mathrm{H}}$ und $\overline{C O P}_{\max }^{\mathrm{H}}$ ist aus ihren Unterschieden ableitbar:

- $C O P_{\max }^{\mathrm{H}}$ kann graphisch und rechnerisch ermittelt werden.

$-\overline{C O P}_{\max }^{\mathrm{H}}$ kann nur graphisch ermittelt werden.

- Das Schema zur Ermittlung von $C O P_{\max }^{\mathrm{H}}$ ist exakt.

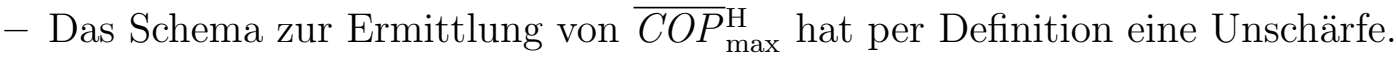

- Die graphische Ermittlung von $C O P_{\max }^{\mathrm{H}}$ ist nicht ohne weiteres invertierbar. Das bedeutet, es nicht einfach möglich z.B. ein maximales $\vartheta_{\mathrm{S}}$ aus einem gegebenem $C O P_{\max }^{\mathrm{H}}$ und einem gegebenem $\vartheta_{\mathrm{Q}} \mathrm{zu}$ ermitteln. Es ist nicht unmöglich, für ein graphisches Verfahren jedoch verhältnismäßig "unkomfortabel".

- Die graphische Ermittlung von $\overline{C O P}_{\max }^{\mathrm{H}}$ ist vollständig invertierbar, da nur ein Diagramm und eine Tabelle benötigt werden.

- Es bietet sich daher an, jede Größe in genau dem Bereich einzusetzen, in dem sie ihre spezifischen Stärken zur Gänze ausspielen kann.

- In der Anwendung ersetzt $\overline{C O P}_{\max }^{\mathrm{H}}$ die Größe $C O P_{\max }^{\mathrm{H}}$. Das bedeutet, dass entweder $\overline{C O P}_{\max }^{\mathrm{H}}$ oder $C O P_{\max }^{\mathrm{H}}$ ermittelt wird. Daher ist in Kapitel 4 die Ermittlung von $\overline{C O P}_{\max }^{\mathrm{H}}$ als Alternative im Rahmen der Ermittlung von $C O P_{\max }^{\mathrm{H}}$ aufgeführt worden.

\subsubsection{Realer COP im Heizbetrieb}

Unter Berücksichtigung der am Anfang von Kapitel 3.3 eingeführten Nomenklatur folgt für den realen COP im Heizbetrieb nach Gleichung (2.9):

$$
C O P_{\text {real }}^{\mathrm{H}}=\frac{\dot{Q}_{34}^{\mathrm{r}}}{\dot{W}_{23}^{\mathrm{r}}}=\frac{\dot{Q}_{\mathrm{N}}^{\mathrm{H}}}{\dot{W}_{23}^{\mathrm{r}}}
$$

Es sei an dieser Stelle darauf hingewiesen, dass der tiefgestellte Index N den Nutzen einer realen Größe (Wärme oder Wärmestrom) kennzeichnet. Der hochgestellte Index r entfällt daher.

Für die folgenden Arbeitsschritte wird festgehalten:

- Ist der real im Kondensator des Wärmepumpenkreislaufs umgesetzte Wärmestrom als Nutzen anzusehen, so gilt:

$$
\dot{Q}_{34}^{\mathrm{r}}=\dot{Q}_{\mathrm{N}}^{\mathrm{H}}
$$

- Ist der real im Verdampfer des Wärmepumpenkreislaufs umgesetzte Wärmestrom als Nutzen anzusehen, so gilt:

$$
\dot{Q}_{12}^{\mathrm{r}}=\dot{Q}_{\mathrm{N}}^{\mathrm{K}}
$$


Unter Verwendung des in Gleichung (2.10) eingeführten Gütegrads folgt für Gleichung (3.34):

$$
\begin{aligned}
\eta_{\mathrm{WP}} & =\frac{C O P_{\mathrm{real}}^{\mathrm{H}}}{C O P_{\max }^{\mathrm{H}}} \\
\longrightarrow C O P_{\mathrm{real}}^{\mathrm{H}} & =\eta_{\mathrm{WP}} C O P_{\max }^{\mathrm{H}}
\end{aligned}
$$

Der reale COP im Heizbetrieb ist eine wichtige Größe im Rahmen der Bewertung von Wärmepumpenkreisläufen. Wie bzw. an welcher Stelle $C O P_{\text {real }}^{\mathrm{H}}$ in ein Bewertungskonzept eingeht, hängt davon ab, in welcher Art und Weise etwaige für die Bewertung benötigte Kennzahlen eingeführt werden. In der vorliegenden Arbeit tritt $C O P_{\text {real }}^{\mathrm{H}}$ zumeist nur bei den technischen Kennzahlen in Erscheinung. Dies ist, mit Vorgriff auf Kapitel 3.4.1, den neu eingeführten wirtschaftlichen Kennzahlen geschuldet.

\section{Erstellung von Diagrammen für $C O P_{\text {real }}^{\mathrm{H}}$}

Auf Basis von:

$$
C O P_{\text {real }}^{\mathrm{H}}=\eta_{\mathrm{WP}} C O P_{\max }^{\mathrm{H}}
$$

kann $C O P_{\text {real }}^{\mathrm{H}}$, wie in Abbildung 3.15 und 3.16 dargestellt, visualisiert werden:

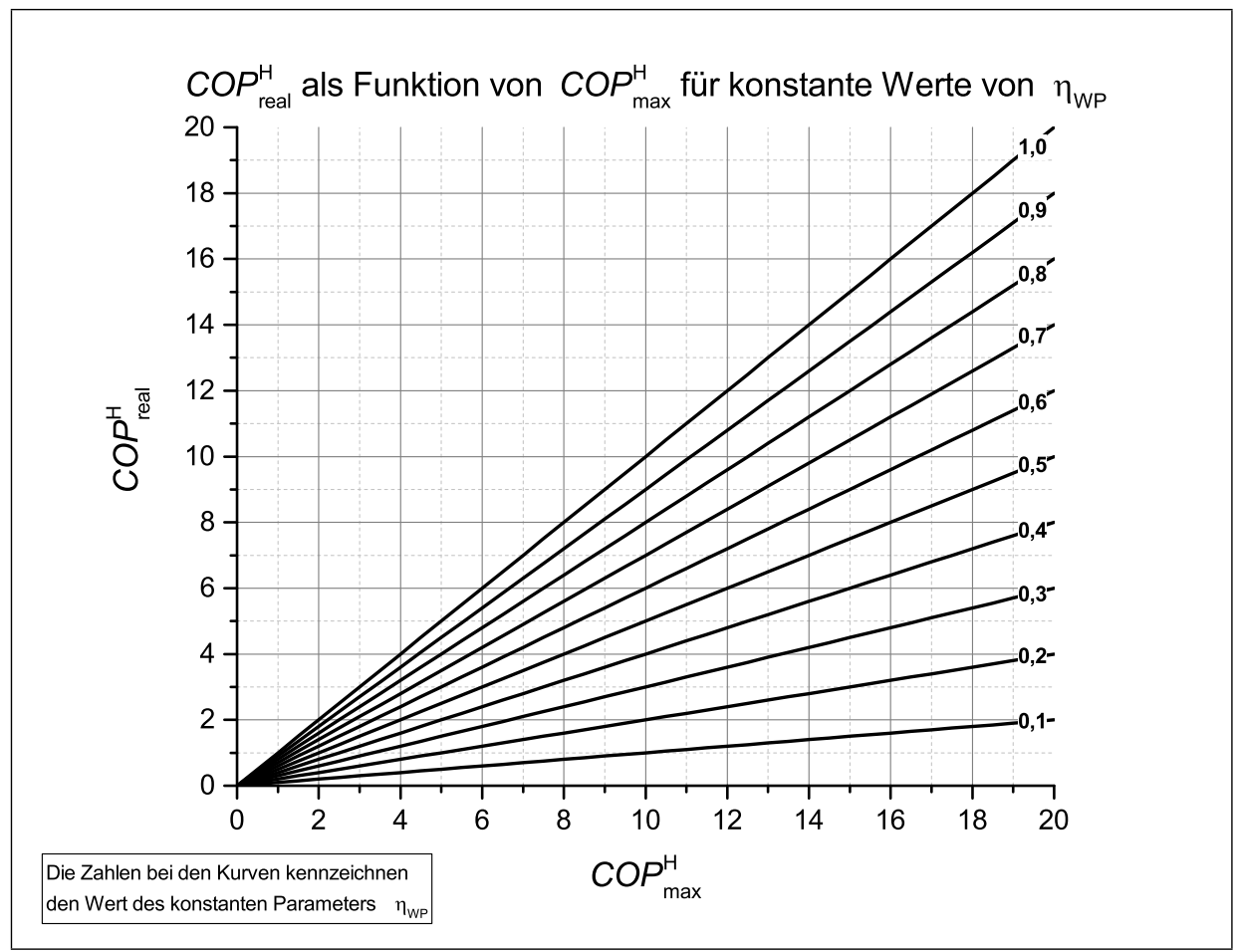

Abbildung 3.15: $C O P_{\text {real }}^{\mathrm{H}}$ als Funktion von $C O P_{\max }^{\mathrm{H}}$ für konstante Werte von $\eta_{\mathrm{WP}}$ 


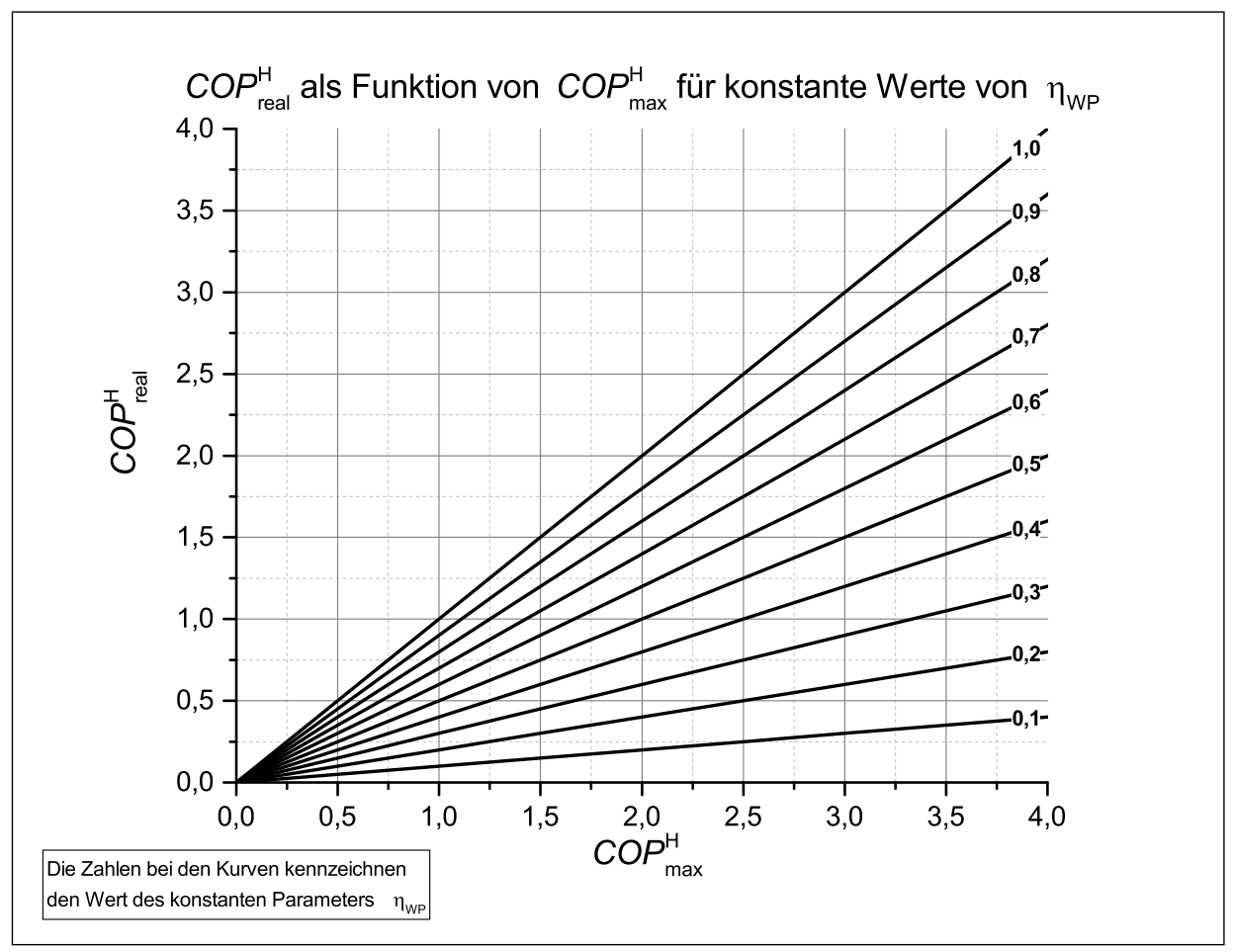

Abbildung 3.16: $C O P_{\text {real }}^{\mathrm{H}}$ als Funktion von $C O P_{\max }^{\mathrm{H}}$ im Intervall $0 \leq C O P_{\max }^{\mathrm{H}} \leq 4$ für konstante Werte von $\eta_{\mathrm{WP}}$

\section{Hinweise zu den Diagrammen zur Ermittlung von $\operatorname{COP}_{\text {real }}^{\mathrm{H}}$}

- Auf der Abszisse in den Abbildungen 3.15 und 3.16 ist $C O P_{\max }^{\mathrm{H}}$ aufgetragen. Als konstanter Parameter wurde $\eta_{\mathrm{WP}}$ gewählt. Um die Ablesbarkeit im Bereich niedriger COP-Werte zu verbessern, zeigt Abbildung 3.16COP ${ }_{\text {real }}^{\mathrm{H}}$ als Funktion von $C O P_{\max }^{\mathrm{H}}$ im Intervall $0 \leq C O P_{\max }^{\mathrm{H}} \leq 4$.

- Der Ableseweg wird im Folgenden am Beispiel von $C O P_{\max }^{\mathrm{H}}$ und $\eta_{\mathrm{WP}}=$ konstant beschrieben:

Es wird eine Vertikale durch $C O P_{\max }^{\mathrm{H}}$ gelegt und mit der korrespondierenden Kurve für $\eta_{\mathrm{WP}}=$ konstant geschnitten. Für den ermittelten Schnittpunkt kann $C O P_{\text {real }}^{\mathrm{H}}$ abgelesen werden.

- Aus $C O P_{\max }^{\mathrm{H}}=3$ und $\eta_{\mathrm{WP}}=0,5$ resultiert beispielsweise $C O P_{\text {real }}^{\mathrm{H}}=1,5$. 


\subsubsection{Realer COP im Heiz- und Kühlbetrieb}

Ist nicht nur der im Kondensator des Wärmepumpenkreislaufs abgegebene Wärmestrom als Nutzen anzusehen, sondern auch jener Wärmestrom, der zwischen den Punkten 1 und 2 in Abbildung 2.1 im Kondensator des Wärmepumpenkreislaufs vom Arbeitsmedium aufgenommen wird, so ist es zweckmäßig den realen COP im Heiz- und Kühlbetrieb zu definieren:

$$
C O P_{\text {real }}^{\mathrm{H}+\mathrm{K}}:=\frac{\dot{Q}_{12}^{\mathrm{r}}+\dot{Q}_{34}^{\mathrm{r}}}{\dot{W}_{23}^{\mathrm{r}}}=\frac{\dot{Q}_{\mathrm{N}}^{\mathrm{K}}+\dot{Q}_{\mathrm{N}}^{\mathrm{H}}}{\dot{W}_{23}^{\mathrm{r}}}
$$

\section{Ermittlung eines Zusammenhangs für $C O P_{\text {real }}^{\mathrm{H}+\mathrm{K}}$}

Unter Verwendung von:

$$
\dot{Q}_{\mathrm{N}}^{\mathrm{K}}+\dot{W}_{23}^{\mathrm{r}}=\dot{Q}_{\mathrm{N}}^{\mathrm{H}}
$$

kann Ergebnis aus Gleichung (3.38) wie folgt umgeformt werden:

$$
\begin{aligned}
C O P_{\text {real }}^{\mathrm{H}+\mathrm{K}} & =\frac{\dot{Q}_{\mathrm{N}}^{\mathrm{K}}+\dot{Q}_{\mathrm{N}}^{\mathrm{H}}}{\dot{W}_{23}^{\mathrm{r}}}=\frac{\dot{Q}_{\mathrm{N}}^{\mathrm{K}}}{\dot{W}_{23}^{\mathrm{r}}}+\frac{\dot{Q}_{\mathrm{N}}^{\mathrm{H}}}{\dot{W}_{23}^{\mathrm{r}}}=\frac{\dot{Q}_{\mathrm{N}}^{\mathrm{K}}}{\dot{W}_{23}^{\mathrm{r}}}+C O P_{\text {real }}^{\mathrm{H}} \\
\longrightarrow & C O P_{\text {real }}^{\mathrm{H}+\mathrm{K}}=\frac{\dot{Q}_{\mathrm{N}}^{\mathrm{H}}-\dot{W}_{23}^{\mathrm{r}}}{\dot{W}_{23}^{\mathrm{r}}}+C O P_{\text {real }}^{\mathrm{H}}=C O P_{\text {real }}^{\mathrm{H}}-1+C O P_{\text {real }}^{\mathrm{H}}
\end{aligned}
$$

Daraus folgt für den realen COP im Heiz- und Kühlbetrieb:

$$
\begin{aligned}
& C O P_{\text {real }}^{\mathrm{H}+\mathrm{K}}=2 C O P_{\text {real }}^{\mathrm{H}}-1 \\
& C O P_{\text {real }}^{\mathrm{H}+\mathrm{K}}=2 \eta_{\mathrm{WP}} C O P_{\text {max }}^{\mathrm{H}}-1
\end{aligned}
$$

Für den realen COP im Heiz- und Kühlbetrieb gilt, wie schon für den realen COP im Heizbetrieb, dass sein kennzahlentechnischer Einfluss von der Art, der in den folgenden Kapiteln eingeführten Kennzahlen, abhängt. Mit Vorgriff auf Kapitel 3.4.1 ist festzustellen, dass der reale COP im Heiz- und Kühlbetrieb sich, trotz seiner Aussagekraft, nicht in den wirtschaftlichen Kennzahlen zu Bewertung der Leistungsfähigkeit eines Wärmepumpenkreislaufs wiederfindet. 
Erstellung von Diagrammen für $C O P_{\text {real }}^{\mathrm{H}+\mathrm{K}}$

Abbildung 3.17 zeigt den realen COP im Heiz und Kühlbetrieb als eine Funktion $C O P_{\text {real }}^{\mathrm{H}}$ :

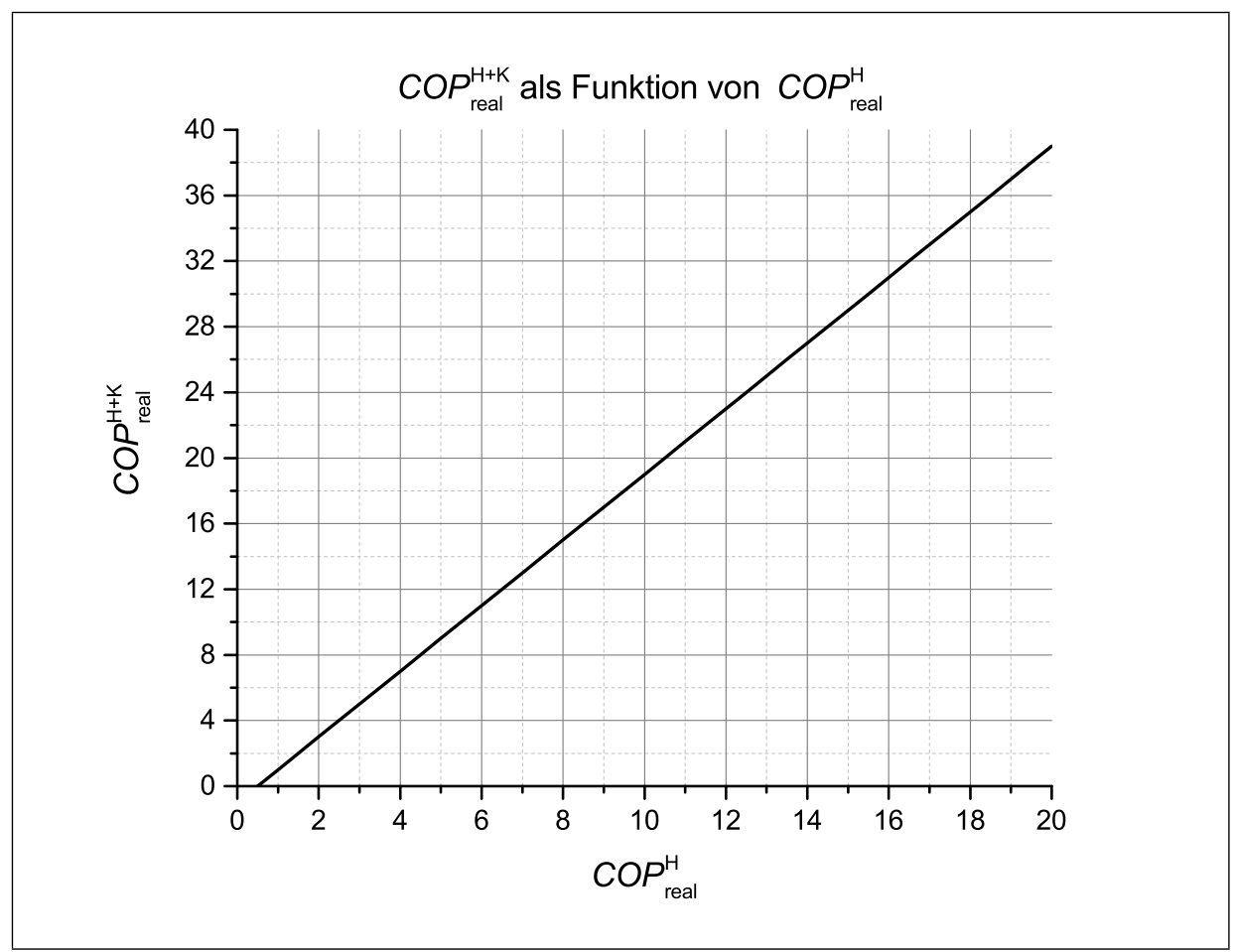

Abbildung 3.17: $C O P_{\text {real }}^{\mathrm{H}+\mathrm{K}}$ als Funktion von $C O P_{\text {real }}^{\mathrm{H}}$

Da es sich bei Abbildung 3.17 um die Darstellung einer Funktion mit nur einer Veränderlichen handelt, ist auf eine gesonderte Erklärung des Diagramms verzichtet worden.

\subsubsection{Quotient der Nutzwärmeströme}

Bei der Ableitung von wirtschaftlichen Kennzahlen hat es sich als nützlich erwiesen, den Quotienten der Nutzwärmeströme $\widehat{Q}$ einzuführen. Er wird definiert mit:

$$
\widehat{Q}:=\frac{\dot{Q}_{\mathrm{N}}^{\mathrm{K}}}{\dot{Q}_{\mathrm{N}}^{\mathrm{H}}}=\frac{\dot{Q}_{12}^{\mathrm{r}}}{\dot{Q}_{34}^{\mathrm{r}}}
$$




\section{Ermittlung eines Zusammenhangs für $\widehat{Q}$}

Mit:

$$
\begin{aligned}
C O P_{\text {real }}^{\mathrm{H}} & =\frac{\dot{Q}_{\mathrm{N}}^{\mathrm{H}}}{\dot{W}_{23}^{\mathrm{r}}} \\
\longrightarrow \dot{Q}_{\mathrm{N}}^{\mathrm{H}} & =\dot{W}_{23}^{\mathrm{r}} C O P_{\text {real }}^{\mathrm{H}}=\dot{W}_{23}^{\mathrm{r}} \eta_{\mathrm{WP}} C O P_{\text {max }}^{\mathrm{H}}
\end{aligned}
$$

und

$$
\dot{Q}_{\mathrm{N}}^{\mathrm{K}}+\dot{W}_{23}^{\mathrm{r}}=\dot{Q}_{\mathrm{N}}^{\mathrm{H}}
$$

folgt für den Quotienten der Nutzwärmeströme:

$$
\begin{aligned}
\dot{Q}_{\mathrm{N}}^{\mathrm{K}} & =\dot{Q}_{\mathrm{N}}^{\mathrm{H}}-\dot{W}_{23}^{\mathrm{r}} \\
\longrightarrow \dot{Q}_{\mathrm{N}}^{\mathrm{K}} & =\dot{W}_{23}^{\mathrm{r}} \eta_{\mathrm{WP}} C O P_{\max }^{\mathrm{H}}-\dot{W}_{23}^{\mathrm{r}} \\
\dot{Q}_{\mathrm{N}}^{\mathrm{K}} & =\dot{W}_{23}^{\mathrm{r}}\left(\eta_{\mathrm{WP}} C O P_{\max }^{\mathrm{H}}-1\right) \\
\longrightarrow \frac{\dot{Q}_{\mathrm{N}}^{\mathrm{K}}}{\dot{Q}_{\mathrm{H}}^{\mathrm{K}}} & =\frac{\dot{W}_{23}^{\mathrm{r}}\left(\eta_{\mathrm{WP}} C O P_{\max }^{\mathrm{H}}-1\right)}{\dot{W}_{23}^{\mathrm{r}} \eta_{\mathrm{WP}} C O P_{\max }^{\mathrm{H}}} \\
& \frac{\dot{Q}_{\mathrm{N}}^{\mathrm{K}}}{\dot{Q}_{\mathrm{N}}^{\mathrm{H}}}=\frac{\eta_{\mathrm{WP}} C O P_{\max }^{\mathrm{H}}-1}{\eta_{\mathrm{WP}} C O P_{\max }^{\mathrm{H}}}
\end{aligned}
$$

Woraus sich der gesuchte Zusammenhang für $\widehat{Q}$ ermitteln lässt:

$$
\begin{aligned}
& \widehat{Q}=\frac{\eta_{\mathrm{WP}} C O P_{\max }^{\mathrm{H}}-1}{\eta_{\mathrm{WP}} C O P_{\max }^{\mathrm{H}}} \\
& \widehat{Q}=\frac{C O P_{\text {real }}^{\mathrm{H}}-1}{C O P_{\text {real }}^{\mathrm{H}}} \\
& \widehat{Q}=1-\frac{1}{C O P_{\text {real }}^{\mathrm{H}}}
\end{aligned}
$$




\section{Erstellung von Diagrammen für $\widehat{\boldsymbol{Q}}$}

Für die Erstellung des Diagramms in Abbildung 3.18 ist der folgende Zusammenhang für $\widehat{Q}$ verwendet worden:

$$
\widehat{Q}=1-\frac{1}{C O P_{\text {real }}^{\mathrm{H}}}
$$

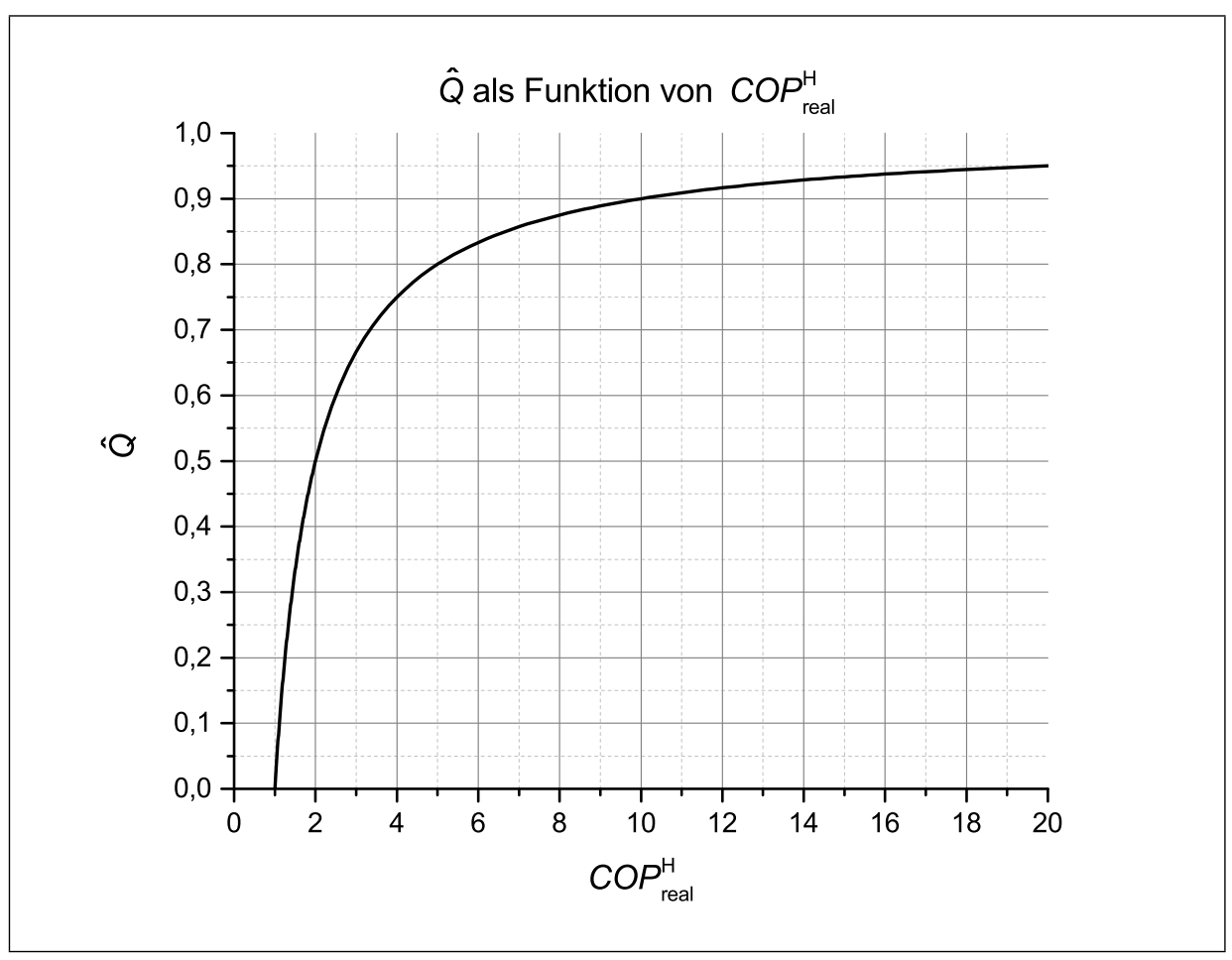

Abbildung 3.18: $\widehat{Q}$ als Funktion von $C O P_{\text {real }}^{\mathrm{H}}$

Da es sich bei Abbildung 3.18 um die Darstellung einer Funktion mit nur einer Veränderlichen handelt, ist auf eine gesonderte Erklärung des Diagramms verzichtet worden.

\subsubsection{COP einer Kälteanlage}

Ersetzt eine Wärmepumpe eine bestehende Kälteanlage, die auf dem Kaltdampf-Kompressions-Prinzip beruht, so wird für die wirtschaftliche Bewertung der Wärmepumpe der $\mathrm{COP}$ der kompensierten Kälteanlage benötigt. Dieser COP, im Folgenden mit $C O P_{\text {real }}^{\mathrm{Kv}}$ angegeben, wird unter Verwendung von:

$$
C O P_{\text {real }}^{\mathrm{Kv}}=\frac{\dot{Q}_{12}^{\mathrm{Kv}}}{\dot{W}_{23}^{\mathrm{Kv}}}
$$

berechnet. 


\subsection{Relative wirtschaftliche Kennzahlen}

In diesem Kapitel werden, aufbauend auf den in Kapitel 3.3 eingeführten technische Kennzahlen, relative wirtschaftliche Kennzahlen für die Bewertung von Wärmepumpen im Heizund Kühlbetrieb erarbeitet. Es gelten weiterhin die in Kapitel 3.2 getätigten Annahmen bzw. Definitionen.

Zusätzlich zu den bereits bekannten Indices, werden in diesem Kapitel die folgenden Indices verwendet:

- Index WP tiefgestellt Größe des Wärmepumpenkreislaufs

- Index kon tiefgestellt Größe der Vergleichstechnologie

- Index el tiefgestellt Kennzeichnung einer elektrischen Größe, z.B. spezifische Kosten

Hinweise: Der tiefgestellte Index kon steht für die Abkürzung des Wortes "konventionell" und kennzeichnet Größen der Vergleichstechnologie. In Kombination mit den bereits in Kapitel 3.3 eingeführten Kennzahlen, ist es möglich zu differenzieren, ob mit der Vergleichstechnologie gekühlt oder geheizt wird. Das Wort "Vergleichstechnologie" ist bewusst gewählt worden, da mit dem hier dokumentierten Bewertungsansatz auch die Wärmebereitstellung mittels z.B. Solarthermie der Wärmepumpe gegenüber gestellt werden kann.

\subsubsection{Wirtschaftlichkeitsgrenze im Heizbetrieb}

Damit eine Wärmepumpe wirtschaftlich sinnvoll in einen Betrieb integriert werden kann, muss zumindest gelten, dass die Kosten zur Bereitstellung einer Nutzwärmemenge $Q_{\mathrm{N}}^{\mathrm{H}}$, im Folgenden auch Wärmebereitstellungskosten genannt, mit einer Wärmepumpe geringer sind als mit der vorhandenen bzw. der zu vergleichenden Technologie. Werden die Wärmebereitstellungskosten mit $G K$ als Funktion von $Q_{\mathrm{N}}^{\mathrm{H}}$ angegeben, so gilt als Bedingung für den wirtschaftlichen Einsatz einer Wärmepumpe:

$$
\frac{G K_{\mathrm{WP}}^{\mathrm{H}}\left(Q_{\mathrm{N}}^{\mathrm{H}}\right)}{G K_{\mathrm{kon}}^{\mathrm{H}}\left(Q_{\mathrm{N}}^{\mathrm{H}}\right)}<1
$$

Hinweis: Die Größe $G K$ steht für die Gesamtkosten und beschreibt den absoluten Betrag der Kosten in einer Währungseinheit. Die in weiterer Folge verwendet Größe $K$ steht für die spezifischen Kosten. So werden beispielsweise die spezifischen Kosten der elektrischen Energie mit $K_{\mathrm{el}}$ angegeben. 


\section{Wärmebereitstellungskosten der Wärmepumpe}

Um eine Nutzwärmemenge $Q_{\mathrm{N}}^{\mathrm{H}}$ bereitzustellen, muss dem Wärmepumpenkreislauf eine von $Q_{\mathrm{N}}^{\mathrm{H}}$ abhängige Menge an Arbeit zugeführt werden, für die daher gelte:

$$
W_{\mathrm{WP}}^{\mathrm{H}}=W_{\mathrm{WP}}^{\mathrm{H}}\left(Q_{\mathrm{N}}^{\mathrm{H}}\right)
$$

Daraus folgt für die Wärmebereitstellungskosten der Wärmepumpe:

$$
G K_{\mathrm{WP}}^{\mathrm{H}}\left(Q_{\mathrm{N}}^{\mathrm{H}}\right)=W_{\mathrm{WP}}^{\mathrm{H}}\left(Q_{\mathrm{N}}^{\mathrm{H}}\right) K_{\mathrm{WP}}^{\mathrm{H}}
$$

Die Größe $K_{\mathrm{WP}}^{\mathrm{H}}$ steht für die spezifischen Kosten einer Wärmepumpe im Heizbetrieb. Nach Gleichung (3.34) gilt:

$$
C O P_{\text {real }}^{\mathrm{H}}=\frac{\dot{Q}_{\mathrm{N}}^{\mathrm{H}}}{\dot{W}_{23}^{\mathrm{r}}}
$$

wobei es sich bei $\dot{Q}_{\mathrm{N}}^{\mathrm{H}}$ und $\dot{W}_{23}^{\mathrm{r}}$ um Prozessgrößen handelt, welche grundsätzlich zeitabhängig sind. Gemäß der Definition von Prozessgrößen [2], beschreiben $\dot{Q}_{\mathrm{N}}^{\mathrm{H}}$ und $\dot{W}_{23}^{\mathrm{r}}$ :

- $\dot{Q}_{\mathrm{N}}^{\mathrm{H}} \quad$ Die aus dem System pro infinitesimalem Zeitintervall abgeführte Wärme.

- $\dot{W}_{23}^{\mathrm{r}}$ Die dem System pro infinitesimalem Zeitintervall zugeführte Arbeit.

In der folgenden Analyse werden die für die Berechnung herangezogen Prozessgrößen über das betrachtete Zeitintervall als konstant angesehen. Es gilt daher:

$$
\begin{gathered}
\dot{Q}_{\mathrm{N}}^{\mathrm{H}} \Delta t=Q_{\mathrm{N}}^{\mathrm{H}} \\
\dot{W}_{23}^{\mathrm{r}} \Delta t=W_{23}^{\mathrm{r}}
\end{gathered}
$$

Mit dem Ergebnis aus Gleichung (3.50) folgt für Gleichung (3.34):

$$
C O P_{\text {real }}^{\mathrm{H}}=\frac{Q_{\mathrm{N}}^{\mathrm{H}}}{W_{23}^{\mathrm{r}}}
$$

was unter Verwendung des Gütegrads durch einfache Umformung in den folgenden Zusammenhang überführt werden kann:

$$
W_{23}^{\mathrm{r}}=\frac{Q_{\mathrm{N}}^{\mathrm{H}}}{\eta_{\mathrm{WP}} C O P_{\max }^{\mathrm{H}}}
$$

Das Resultat aus Gleichung (3.52) beschreibt die Arbeit die dem System zuzuführen ist, um eine gewisse Menge an Nutzwärme, bei gegebenem Gütegrad und gegebenem maximalen COP im Heizbetrieb, bereitzustellen. 
Unter Berücksichtigung der in Kapitel 3.2 formulierten Annahme, dass der Gütegrad auch jegliche Konversionsverluste auf der elektrischen Seite des Wärmepumpenkreislaufs abdeckt, folgt für die dem System zuzuführende Arbeit:

$$
\begin{aligned}
W_{23}^{\mathrm{r}} & =W_{\mathrm{WP}}^{\mathrm{H}}\left(Q_{\mathrm{N}}^{\mathrm{H}}\right) \\
\longrightarrow W_{\mathrm{WP}}^{\mathrm{H}}\left(Q_{\mathrm{N}}^{\mathrm{H}}\right) & =\frac{Q_{\mathrm{N}}^{\mathrm{H}}}{\eta_{\mathrm{WP}} C O P_{\max }^{\mathrm{H}}}
\end{aligned}
$$

Mit der Erkenntnis aus Gleichung (3.53) lassen sich die Wärmebereitstellungskosten der Wärmepumpe, formuliert in Gleichung (3.49), wie folgt berechnen:

$$
\begin{aligned}
G K_{\mathrm{WP}}^{\mathrm{H}}\left(Q_{\mathrm{N}}^{\mathrm{H}}\right) & =W_{\mathrm{WP}}^{\mathrm{H}}\left(Q_{\mathrm{N}}^{\mathrm{H}}\right) K_{\mathrm{WP}}^{\mathrm{H}} \\
\longrightarrow G K_{\mathrm{WP}}^{\mathrm{H}}\left(Q_{\mathrm{N}}^{\mathrm{H}}\right) & =\frac{Q_{\mathrm{N}}^{\mathrm{H}}}{\eta_{\mathrm{WP}} C O P_{\max }^{\mathrm{H}}} K_{\mathrm{WP}}^{\mathrm{H}}
\end{aligned}
$$

\section{Wärmebereitstellungskosten der Vergleichstechnologie}

Um eine Nutzwärmemenge $Q_{\mathrm{N}}^{\mathrm{H}}$ bereitzustellen, muss der Vergleichstechnologie eine von $Q_{\mathrm{N}}^{\mathrm{H}}$ abhängige Energiemenge $Q_{\mathrm{kon}}^{\mathrm{H}}$ zugeführt werden, für welche die folgende Bedingung gelten muss:

$$
Q_{\mathrm{kon}}^{\mathrm{H}}\left(Q_{\mathrm{N}}^{\mathrm{H}}\right) \geq Q_{\mathrm{N}}^{\mathrm{H}}
$$

Für die Wärmebereitstellungskosten der Vergleichstechnologie gilt daher:

$$
G K_{\mathrm{kon}}^{\mathrm{H}}\left(Q_{\mathrm{N}}^{\mathrm{H}}\right)=Q_{\mathrm{kon}}^{\mathrm{H}}\left(Q_{\mathrm{N}}^{\mathrm{H}}\right) K_{\mathrm{kon}}^{\mathrm{H}}
$$

Um ein möglichst allgemeines Konzept für die Berechnung der Wärmebereitstellungskosten der Vergleichstechnologie zur Verfügung zu haben, wird folgender Ansatz gewählt:

- Es wird der Konversionswirkungsgrad der Vergleichstechnologie $\eta_{\text {kon }}$ eingeführt. Für ihn gelte:

$$
\eta_{\mathrm{kon}}:=\frac{Q_{\mathrm{N}}}{Q_{\mathrm{kon}}}
$$


Hinweise zum Konversionswirkungsgrad $\eta_{\text {kon }}$ :

- Der Konversionswirkungsgrad im Heizbetrieb $\eta_{\mathrm{kon}}^{\mathrm{H}}$ hat eine große Bedeutung, da er die Umrechnung von Primärenergie in Nutzwärme erlaubt. Diese Fähigkeit ist insofern von Bedeutung, da die Primärenergie beim Energieversorgungsunternehmen eingekauft wird. Dieser Umstand findet, mit Vorgriff, in Gleichung (3.59) Verwendung.

- Der Konversionswirkungsgrad im Kühlbetrieb $\eta_{\mathrm{kon}}^{\mathrm{K}}$ ist in der Lage ähnliche Phänomene wie beim Konversionswirkungsgrad im Heizbetrieb zu berücksichtigen. Seine eigentliche Stärke ist jedoch die Wahrung eines konsistenten und damit nachvollziehbaren Berechnungsansatzes für die relative Einsparung im Heiz- und Kühlbetrieb. Detaillierte Ausführungen dazu befinden sich in Kapitel 3.4.3.

- Wird $Q_{\text {kon }}$ keiner Konversion unterzogen z.B. im Falle des Zukaufs von Dampf, welcher direkt für die Wärmebereitstellung verwendet wird, so ist $\eta_{\mathrm{kon}}=1 \mathrm{zu}$ setzen.

Aus Gleichung (3.57) folgt für den Heizfall:

$$
\eta_{\mathrm{kon}}^{\mathrm{H}}=\frac{Q_{\mathrm{N}}^{\mathrm{H}}}{Q_{\mathrm{kon}}^{\mathrm{H}}}
$$

Das Resultat aus Gleichung (3.58) eingesetzt in Gleichung (3.56):

$$
\begin{aligned}
G K_{\mathrm{kon}}^{\mathrm{H}}\left(Q_{\mathrm{N}}^{\mathrm{H}}\right) & =Q_{\mathrm{kon}}^{\mathrm{H}}\left(Q_{\mathrm{N}}^{\mathrm{H}}\right) K_{\mathrm{kon}}^{\mathrm{H}} \\
\longrightarrow G K_{\mathrm{kon}}^{\mathrm{H}}\left(Q_{\mathrm{N}}^{\mathrm{H}}\right) & =\frac{Q_{\mathrm{N}}^{\mathrm{H}}}{\eta_{\mathrm{kon}}^{\mathrm{H}}} K_{\mathrm{kon}}^{\mathrm{H}}
\end{aligned}
$$

ergibt den gesuchten Zusammenhang zur Berechnung der Wärmebereitstellungskosten der Vergleichstechnologie. 


\section{Ermittlung der Wirtschaftlichkeitsgrenze}

Die Bedingung für den wirtschaftlichen Einsatz einer Wärmepumpe im Heizbetrieb ist nach Gleichung (3.47) gegeben durch:

$$
\frac{G K_{\mathrm{WP}}^{\mathrm{H}}\left(Q_{\mathrm{N}}^{\mathrm{H}}\right)}{G K_{\mathrm{kon}}^{\mathrm{H}}\left(Q_{\mathrm{N}}^{\mathrm{H}}\right)}<1
$$

was unter Berücksichtigung von Gleichung (3.54) und Gleichung (3.59) in:

$$
\begin{aligned}
& \frac{G K_{\mathrm{WP}}^{\mathrm{H}}\left(Q_{\mathrm{N}}^{\mathrm{H}}\right)}{G K_{\mathrm{kon}}^{\mathrm{H}}\left(Q_{\mathrm{N}}^{\mathrm{H}}\right)}=\frac{\frac{Q_{\mathrm{N}}^{\mathrm{H}}}{\eta_{\mathrm{WP}} C O P_{\max }^{\mathrm{H}}} K_{\mathrm{WP}}^{\mathrm{H}}}{\frac{Q_{\mathrm{N}}^{\mathrm{H}}}{\eta_{\mathrm{kon}}^{\mathrm{H}}} K_{\mathrm{kon}}^{\mathrm{H}}}<1 \\
& \frac{G K_{\mathrm{WP}}^{\mathrm{H}}\left(Q_{\mathrm{N}}^{\mathrm{H}}\right)}{G K_{\mathrm{kon}}^{\mathrm{H}}\left(Q_{\mathrm{N}}^{\mathrm{H}}\right)}=\frac{K_{\mathrm{WP}}^{\mathrm{H}}}{K_{\mathrm{kon}}^{\mathrm{H}}} \frac{\eta_{\mathrm{kon}}^{\mathrm{H}}}{\eta_{\mathrm{WP}}} \frac{1}{C O P_{\max }^{\mathrm{H}}}<1
\end{aligned}
$$

überführt werden kann.

Daraus folgt für die Bedingung des wirtschaftlichen Einsatzes einer Wärmepumpe im Heizbetrieb:

$$
\frac{K_{\mathrm{WP}}^{\mathrm{H}}}{K_{\mathrm{kon}}^{\mathrm{H}}} \frac{\eta_{\mathrm{kon}}^{\mathrm{H}}}{\eta_{\mathrm{WP}}}<C O P_{\max }^{\mathrm{H}}
$$

mit der Wirtschaftlichkeitsgrenze von:

$$
\frac{K_{\mathrm{WP}}^{\mathrm{H}}}{K_{\mathrm{kon}}^{\mathrm{H}}} \frac{\eta_{\mathrm{kon}}^{\mathrm{H}}}{\eta_{\mathrm{WP}}}=C O P_{\max }^{\mathrm{H}}
$$

\section{Einführung des Gesamteinflussfaktors}

Gilt es allgemein, die Gesamtkosten zweier Technologien zu vergleichen, so ist die Technologie A der Technologie B wirtschaftlich überlegen, wenn zur Bereitstellung der gleichen Nutzwärmemenge gilt:

$$
\frac{Q_{\mathrm{N}}^{\mathrm{H}} \frac{K_{\mathrm{A}}}{\eta_{\mathrm{A}}}}{Q_{\mathrm{N}}^{\mathrm{H}} \frac{K_{\mathrm{B}}}{\eta_{\mathrm{B}}}}<1 \longrightarrow \frac{K_{\mathrm{A}}}{K_{\mathrm{B}}} \frac{\eta_{\mathrm{B}}}{\eta_{\mathrm{A}}}<1
$$

Das in Gleichung (3.63) vorkommende Verhältnis:

$$
\frac{K_{\mathrm{A}}}{K_{\mathrm{B}}} \frac{\eta_{\mathrm{B}}}{\eta_{\mathrm{A}}}
$$

kann interpretiert werden, als der Quotient aus den spezifischen Kosten, welche jeweils um einen festen, mit der Technologie verbundenen Faktor, erhöht werden. Dieser Quotient wird im Folgenden als Gesamteinflussfaktors $F_{\mathrm{B}}^{\mathrm{A}}$ bezeichnet:

$$
F_{\mathrm{B}}^{\mathrm{A}}:=\frac{K_{\mathrm{A}}}{K_{\mathrm{B}}} \frac{\eta_{\mathrm{B}}}{\eta_{\mathrm{A}}}
$$


Der Vergleich einer Wärmepumpe mit einer anderen Technologie auf Basis des Gesamteinflussfaktors ist nicht direkt möglich, da $C O P_{\max }^{\mathrm{H}}$ nicht vorkommt. Versteht man $C O P_{\max }^{\mathrm{H}}$ jedoch als eine Größe, die nicht von der Technologie, sondern von der Prozessführung abhängt, so kann der Gesamteinflussfaktor auch auf Fragestellungen, bei denen eine Wärmepumpe vorkommt, zum Einsatz kommen.

Die Anwendung der bisher verwendeten Art der Indexierung würde beim Gesamteinflussfaktor an ihre Grenzen stoßen, da neben den Technologien auch noch die verschiedenen Fälle, z.B. Heizen oder Kühlen, berücksichtigt werden müssten. Um die Übersichtlichkeit zu erhöhen, werden drei Gesamteinflussfaktoren definiert:

- Gesamteinflussfaktor im Heizbetrieb

$$
F_{\text {ges }}^{\mathrm{H}}:=\frac{K_{\mathrm{WP}}^{\mathrm{H}}}{K_{\mathrm{kon}}^{\mathrm{H}}} \frac{\eta_{\mathrm{kon}}^{\mathrm{H}}}{\eta_{\mathrm{WP}}}
$$

- Gesamteinflussfaktor im Kühlbetrieb

$$
F_{\mathrm{ges}}^{\mathrm{K}}:=\frac{K_{\mathrm{WP}}^{\mathrm{K}}}{K_{\mathrm{kon}}^{\mathrm{K}}} \frac{\eta_{\mathrm{kon}}^{\mathrm{K}}}{\eta_{\mathrm{WP}}}
$$

\section{- Gesamteinflussfaktor im Heiz- und Kühlbetrieb}

$$
\begin{aligned}
F_{\mathrm{ges}}^{\mathrm{H}+\mathrm{K}}:=\frac{F_{\mathrm{ges}}^{\mathrm{H}}}{F_{\mathrm{ges}}^{\mathrm{K}}} \\
\longrightarrow F_{\mathrm{ges}}^{\mathrm{H}+\mathrm{K}}=\frac{K_{\mathrm{kon}}^{\mathrm{K}}}{K_{\mathrm{kon}}^{\mathrm{H}}} \frac{\eta_{\mathrm{kon}}^{\mathrm{H}}}{\eta_{\mathrm{kon}}^{\mathrm{K}}}
\end{aligned}
$$

Es sei an dieser Stelle darauf hingewiesen, dass die Gesamteinflussfaktoren nach Gleichung (3.66) bis (3.68) definiert wurden, um in den späteren Berechnungsschritten auf einheitlich eingeführte Größen zurückgreifen zu können. Mit Ausnahme des Gesamteinflussfaktors im Heizbetrieb, welcher, wie in den späteren Ausführung gezeigt wird, auch als Grenze der Wirtschaftlichkeit im Heizbetrieb interpretiert werden kann, haben daher die hier definierten Größen den Charakter einer Hilfsgröße.

Ein Vorteil, der sich aus der einheitlichen Einführung der Gesamteinflussfaktoren ergibt, ist die Tatsache, dass die Gesamteinflussfaktoren aus einem Satz an Diagrammen vom Typ $F_{\mathrm{B}}^{\mathrm{A}}$ ermitteln werden können. 


\section{Anwendung des Gesamteinflussfaktor im Heizbetrieb}

Die Bedingung für den wirtschaftlichen Einsatz einer Wärmepumpe nach Gleichung (3.61) lässt sich unter Verwendung des Gesamteinflussfaktor im Heizbetrieb nach Gleichung (3.66) wie folgt darstellen:

$$
\begin{gathered}
\frac{K_{\mathrm{WP}}^{\mathrm{H}}}{K_{\mathrm{kon}}^{\mathrm{H}}} \frac{\eta_{\mathrm{kon}}^{\mathrm{H}}}{\eta_{\mathrm{WP}}}<C O P_{\max }^{\mathrm{H}} \\
\longrightarrow F_{\text {ges }}^{\mathrm{H}}<C O P_{\text {max }}^{\mathrm{H}}
\end{gathered}
$$

Mit der oberen Grenze für $F_{\text {ges }}^{\mathrm{H}}$ von:

$$
\left.F_{\text {ges }}^{\mathrm{H}}\right|_{\max }=C O P_{\max }^{\mathrm{H}}
$$

Die in Gleichung (3.70) formulierte Grenze ist die Wirtschaftlichkeitsgrenze einer Wärmepumpe im Heizbetrieb. Ist der Gesamteinflussfaktor größer als der maximale COP im Heizbetrieb, so ist der wirtschaftliche Betrieb einer Wärmepumpe im Heizbetrieb nicht möglich.

\section{Erstellung von Diagrammen für $\boldsymbol{F}_{\mathrm{B}}^{\mathrm{A}}$}

Die Berechnungsvorschrift für $F_{\mathrm{B}}^{\mathrm{A}}$ ist nach Gleichung (3.65) gegeben durch:

$$
F_{\mathrm{B}}^{\mathrm{A}}=\frac{K_{\mathrm{A}}}{K_{\mathrm{B}}} \frac{\eta_{\mathrm{B}}}{\eta_{\mathrm{A}}}
$$

Das Verhältnis der spezifischen Kosten $K_{\mathrm{A}}$ zu $K_{\mathrm{B}}$ ist eine wichtige und häufig variable Größe bei der Bewertung von verschiedenen Technologien. Der Quotient aus $\eta_{\mathrm{B}}$ und $\eta_{\mathrm{A}}$ hingegen, kann zumeist als konstant angenommen werden. Es ist daher festgelegt worden, Diagramme für $F_{\mathrm{B}}^{\mathrm{A}}$ als Funktion von $K_{\mathrm{A}}$ zu $K_{\mathrm{B}}$ für konstante Werte des Quotienten aus $\eta_{\mathrm{B}}$ und $\eta_{\mathrm{A}} \mathrm{zu}$ ermitteln.

Im Rahmen der Erstellung von Diagrammen für $F_{\mathrm{B}}^{\mathrm{A}}$ sind die folgenden Schritte durchlaufen worden:

1. Ermittlung des relevanten Intervalls für $F_{\mathrm{B}}^{\mathrm{A}}$.

2. Ableitung von Diagrammen für den Quotienten aus $\eta_{\mathrm{B}}$ und $\eta_{\mathrm{A}}$.

3. Erstellung von Diagrammen für $F_{\mathrm{B}}^{\mathrm{A}}$. 
Ermittlung des relevanten Intervalls für $F_{\mathrm{B}}^{\mathrm{A}}$

Diagramme vom Typ $F_{\mathrm{B}}^{\mathrm{A}}$ werden in der vorliegenden Arbeit zur graphischen Bestimmung von $F_{\text {ges }}^{\mathrm{H}}$ und $F_{\text {ges }}^{\mathrm{H}+\mathrm{K}}$ benötigt. Die Größe $F_{\text {ges }}^{\mathrm{K}}$ dient nur der nachvollziehbaren Einführung von $F_{\text {ges }}^{\mathrm{H}+\mathrm{K}}$ und ist daher nicht aus Diagrammen abzulesen.

Die Ermittlung des darzustellenden Intervalls von $F_{\mathrm{B}}^{\mathrm{A}}$, um sowohl $F_{\text {ges }}^{\mathrm{H}}$ als auch $F_{\text {ges }}^{\mathrm{H}+\mathrm{K}}$ aus ein und dem selben Diagramm ablesen zu können, beruht auf den folgenden Überlegungen:

Nach Gleichung (3.70) ist $F_{\text {ges }}^{\mathrm{H}}$ nach oben mit $C O P_{\max }^{\mathrm{H}}$ beschränkt. Der obere Grenzwert für $C O P_{\max }^{\mathrm{H}}$ liegt laut Abbildung 3.10 bei ungefähr 20. Daraus folgt für die obere Grenze von $F_{\mathrm{B}}^{\mathrm{A}}$, dass diese ebenfalls ungefähr 20 beträgt, wenn:

$$
F_{\text {ges }}^{\mathrm{K}}>1
$$

erfüllt ist. Diese Forderung ist dadurch begründet, dass die Erfüllung der Bedingung aus Gleichung (3.71) gewährleistet, dass $F_{\text {ges }}^{\mathrm{H}+\mathrm{K}}$ kleiner als $F_{\text {ges }}^{\mathrm{H}}$ ist, da für $F_{\text {ges }}^{\mathrm{H}+\mathrm{K}}$ gilt:

$$
F_{\mathrm{ges}}^{\mathrm{H}+\mathrm{K}}=\frac{F_{\mathrm{ges}}^{\mathrm{H}}}{F_{\mathrm{ges}}^{\mathrm{K}}}
$$

Eine tiefergehende Diskussion der verschiedenen Fälle, die sich bei der Berechnung von $F_{\text {ges }}^{\mathrm{K}}$ ergeben und welche Auswirkung diese Fälle auf den Wertebereich von $F_{\text {ges }}^{\mathrm{K}}$ haben, ist den Ausführungen in Kapitel 3.4.3 vorbehalten, da das Thema Heizen und Kühlen dort einheitlich behandelt wird. An dieser Stelle wird daher, nur mit Vorgriff auf Kapitel 3.4.3, festgestellt, dass das Intervall:

$$
0 \leq F_{\mathrm{B}}^{\mathrm{A}} \leq 20
$$

sowohl für die graphische Ermittlung von $F_{\text {ges }}^{\mathrm{H}}$ als auch $F_{\text {ges }}^{\mathrm{H}+\mathrm{K}}$ ausreichend ist.

Ableitung von Diagrammen für den Quotienten aus $\eta_{\mathrm{B}}$ und $\eta_{\mathrm{A}}$ Abbildung 3.19 und 3.20 zeigen den Quotient aus $\eta_{\mathrm{B}}$ und $\eta_{\mathrm{A}}$ für konstante Werte von $\eta_{\mathrm{B}}$.

Abbildung 3.19 repräsentiert den technisch und wirtschaftlich relevanten Bereich von $\eta_{\mathrm{A}} \geq$ 0,2. Die Darstellung in Abbildung 3.20 für $0,05 \leq \eta_{\mathrm{A}} \leq 0,2$ ist rein der Vollständigkeit halber aufgeführt.

Aus Abbildung 3.19 folgt der für die weitere Analyse in Abbildung 3.21 verwendete Wertebereich von $\eta_{\mathrm{B}} / \eta_{\mathrm{A}}$ mit $0,5 \leq \eta_{\mathrm{B}} / \eta_{\mathrm{A}} \leq 5$. 


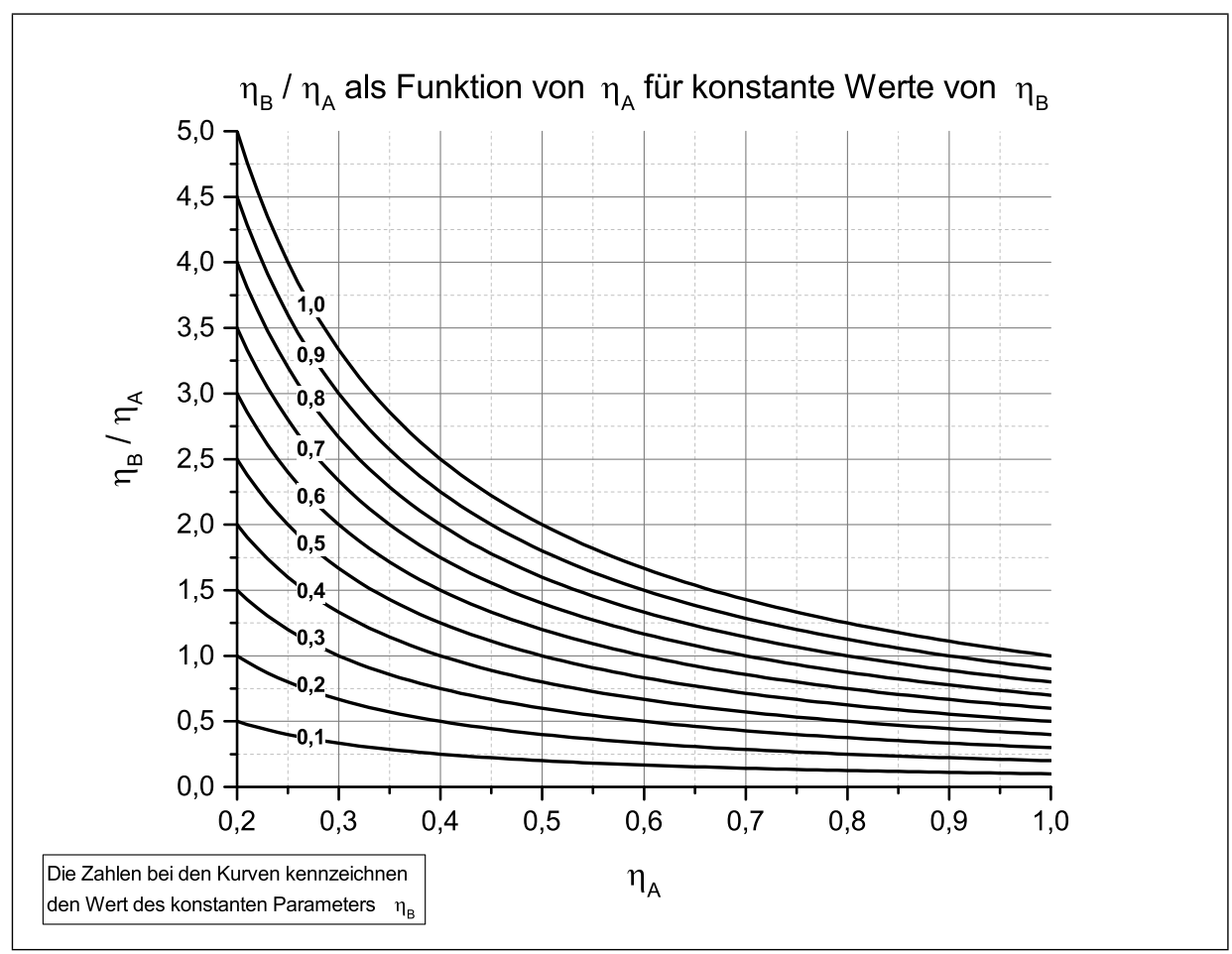

Abbildung 3.19: Quotient aus $\eta_{\mathrm{B}}$ und $\eta_{\mathrm{A}}$ im Intervall $0,2 \leq \eta_{\mathrm{A}} \leq 1$ für konstante Werte von $\eta_{\mathrm{B}}$

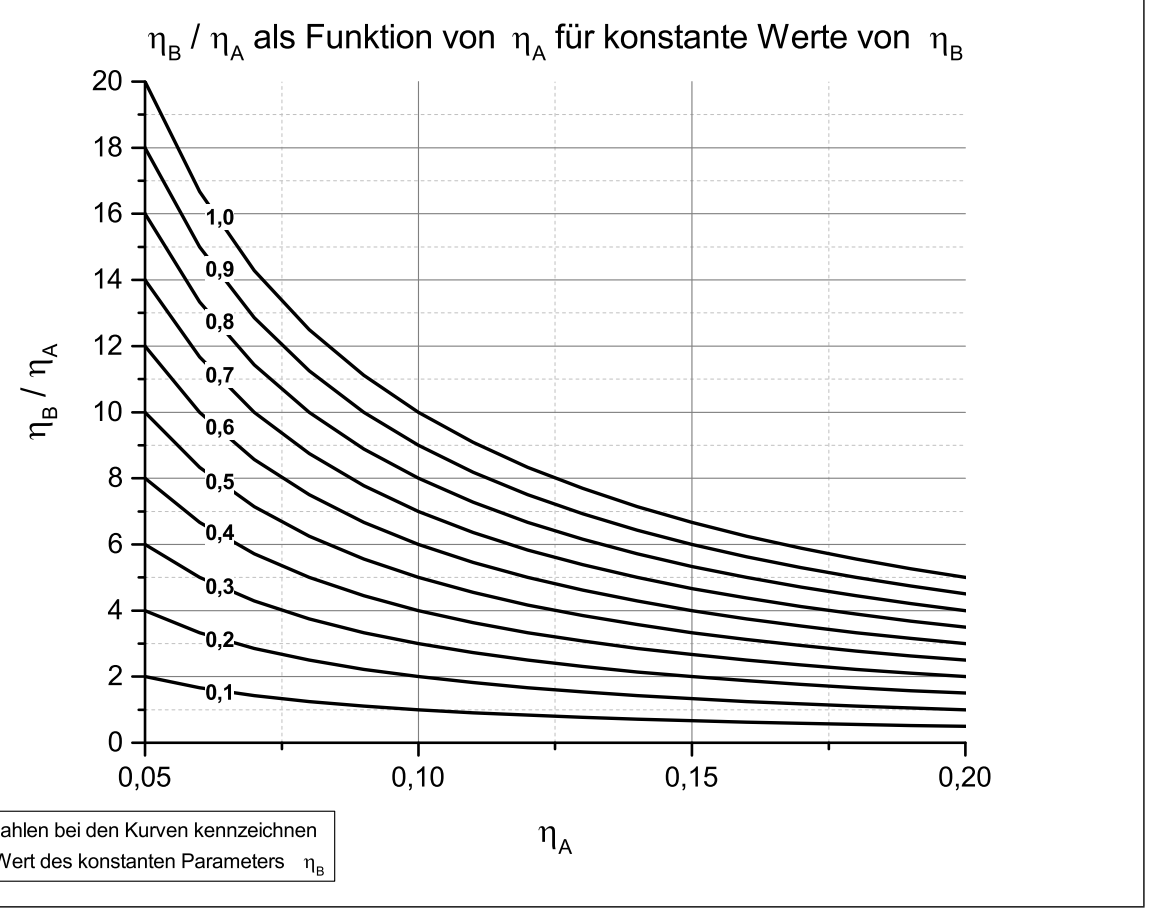

Abbildung 3.20: Quotient aus $\eta_{\mathrm{B}}$ und $\eta_{\mathrm{A}}$ im Intervall $0,05 \leq \eta_{\mathrm{A}} \leq 0,2$ für konstante Werte von $\eta_{\mathrm{B}}$ 
$\underline{\text { Hinweise zu den Diagrammen zur Ermittlung des Quotienten aus } \eta_{\mathrm{B}} \text { und } \eta_{\mathrm{A}}}$

- Auf der Abszisse in Abbildung 3.19 und 3.20 ist $\eta_{\mathrm{A}}$ aufgetragen. Als konstanter Parameter wurde $\eta_{\mathrm{B}}$ gewählt.

- Der Ableseweg wird im Folgenden am Beispiel von $\eta_{\mathrm{A}}$ und $\eta_{\mathrm{B}}=$ konstant beschrieben: Es wird eine Vertikale durch $\eta_{\mathrm{A}}$ gelegt und mit der korrespondierenden Kurve für $\eta_{\mathrm{B}}=$ konstant geschnitten. Für den ermittelten Schnittpunkt kann das Verhältnis von $\eta_{\mathrm{B}} \mathrm{zu} \eta_{\mathrm{A}}$ abgelesen werden.

- Für $\eta_{\mathrm{A}}=0,5$ und $\eta_{\mathrm{B}}=0,9$ folgt beispielsweise $\eta_{\mathrm{B}} / \eta_{\mathrm{A}}=1,8$.

Erstellung von Diagrammen für $F_{\mathrm{B}}^{\mathrm{A}}$

Abbildung 3.21 zeigt $F_{\mathrm{B}}^{\mathrm{A}}$ als Funktion von $K_{\mathrm{A}}$ zu $K_{\mathrm{B}}$ im Intervall $0 \leq F_{\mathrm{B}}^{\mathrm{A}} \leq 20$ für konstante Werte des Quotienten aus $\eta_{\mathrm{B}}$ und $\eta_{\mathrm{A}}$. Nachdem der Gesamteinflussfaktor im Heiz- und Kühlbetrieb häufig für kleine Werte von $K_{\mathrm{A}}$ zu $K_{\mathrm{B}}$ zu ermitteln ist, zeigt Abbildung 3.22 $F_{\mathrm{B}}^{\mathrm{A}}$ im Intervall $0 \leq K_{\mathrm{A}} / K_{\mathrm{B}} \leq 2$ und $0 \leq F_{\mathrm{B}}^{\mathrm{A}} \leq 4$.

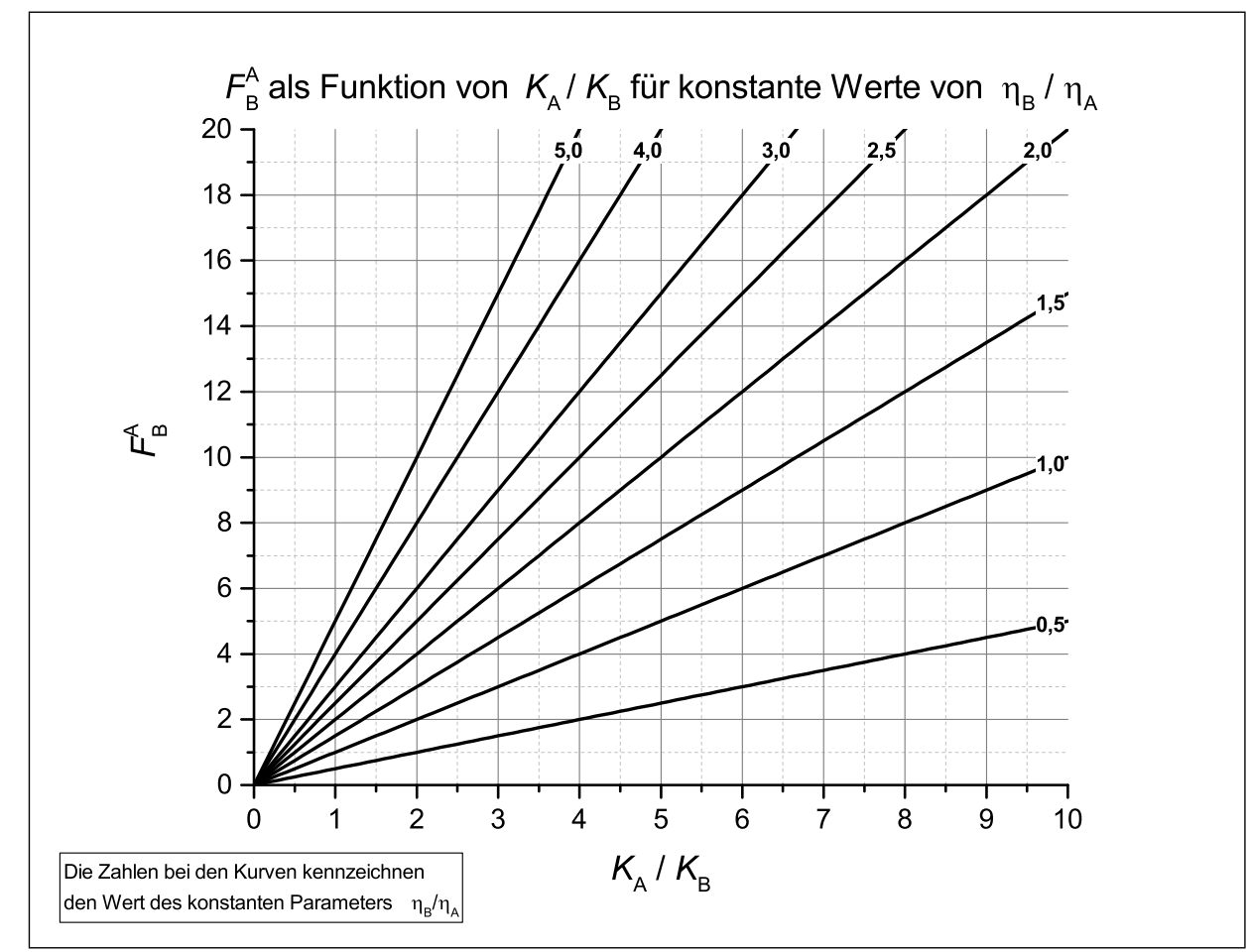

Abbildung 3.21: $F_{\mathrm{B}}^{\mathrm{A}}$ als Funktion von $K_{\mathrm{A}}$ zu $K_{\mathrm{B}}$ im Intervall $0 \leq F_{\mathrm{B}}^{\mathrm{A}} \leq 20$ für konstante Werte von $\eta_{\mathrm{B}} / \eta_{\mathrm{A}}$ 


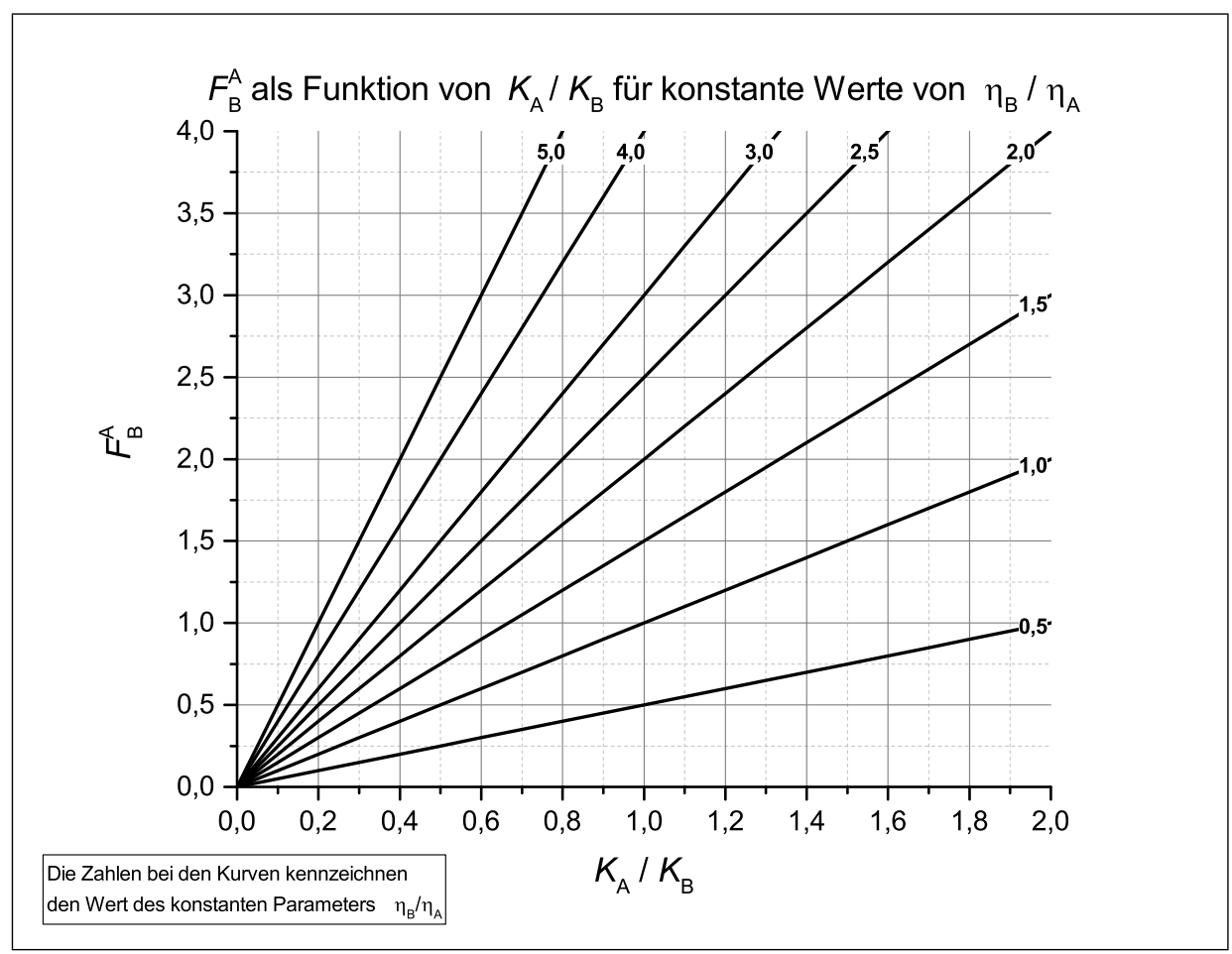

Abbildung 3.22: $F_{\mathrm{B}}^{\mathrm{A}}$ als Funktion von $K_{\mathrm{A}}$ zu $K_{\mathrm{B}}$ im Intervall $0 \leq K_{\mathrm{A}} / K_{\mathrm{B}} \leq 2$ und $0 \leq$ $F_{\mathrm{B}}^{\mathrm{A}} \leq 4$ für konstante Werte von $\eta_{\mathrm{B}} / \eta_{\mathrm{A}}$

Hinweise zu den Diagrammen zur Ermittlung von $F_{\mathrm{B}}^{\mathrm{A}}$

- Auf der Abszisse in Abbildung 3.21 und 3.22 ist $K_{\mathrm{A}} / K_{\mathrm{B}}$ aufgetragen. Als konstanter Parameter wurde $\eta_{\mathrm{B}} / \eta_{\mathrm{A}}$ gewählt.

- Der Ableseweg wird im Folgenden am Beispiel von $K_{\mathrm{A}} / K_{\mathrm{B}}$ und $\eta_{\mathrm{B}} / \eta_{\mathrm{A}}=$ konstant beschrieben:

Es wird eine Vertikale durch $K_{\mathrm{A}} / K_{\mathrm{B}}$ gelegt und mit der korrespondierenden Kurve für $\eta_{\mathrm{B}} / \eta_{\mathrm{A}}=$ konstant geschnitten. Für den ermittelten Schnittpunkt kann $F_{\mathrm{B}}^{\mathrm{A}}$ abgelesen werden.

- Für $K_{\mathrm{A}} / K_{\mathrm{B}}=1,2$ und $\eta_{\mathrm{B}} / \eta_{\mathrm{A}}=2$ kann beispielsweise $F_{\mathrm{B}}^{\mathrm{A}}=2,4$ abgelesen werden. 
Ermittlung von $\boldsymbol{F}_{\text {ges }}^{\mathrm{H}}$ aus den Diagrammen von $\boldsymbol{F}_{\mathrm{B}}^{\mathrm{A}}$

Um $F_{\text {ges }}^{\mathrm{H}}$ aus den Diagrammen für $F_{\mathrm{B}}^{\mathrm{A}}$ ablesen zu können, sind zuerst die für die Ablesung notwendigen Größen zu ermitteln. Es gilt:

$$
\begin{aligned}
F_{\mathrm{B}}^{\mathrm{A}} & =\frac{K_{\mathrm{A}}}{K_{\mathrm{B}}} \frac{\eta_{\mathrm{B}}}{\eta_{\mathrm{A}}} \\
F_{\mathrm{ges}}^{\mathrm{H}} & =\frac{K_{\mathrm{WP}}^{\mathrm{H}}}{K_{\mathrm{kon}}^{\mathrm{H}}} \frac{\eta_{\mathrm{kon}}^{\mathrm{H}}}{\eta_{\mathrm{WP}}}
\end{aligned}
$$

Daraus folgt für den Ableseprozess:

$$
\begin{aligned}
\eta_{\mathrm{A}} & =\eta_{\mathrm{WP}} & \eta_{\mathrm{B}} & =\eta_{\mathrm{kon}}^{\mathrm{H}} \\
K_{\mathrm{A}} & =K_{\mathrm{WP}}^{\mathrm{H}} & K_{\mathrm{B}} & =K_{\mathrm{kon}}^{\mathrm{H}}
\end{aligned}
$$

Unter Verwendung der in Gleichung (3.73) angegebenen Zusammenhänge, wird in einem ersten Schritt der Quotient aus $\eta_{\mathrm{kon}}^{\mathrm{H}}$ und $\eta_{\mathrm{WP}}$ aus Abbildung 3.19 bzw. 3.20 ermittelt. Im Anschluss kann der gesuchte Wert für $F_{\text {ges }}^{\mathrm{H}}$ aus Abbildung 3.21 bzw. 3.22 abgelesen werden.

\subsubsection{Relative Energiekosteneinsparung im Heizbetrieb}

In Kapitel 3.4.1 wurden die Wärmebereitstellungskosten einer Nutzwärmemenge $Q_{\mathrm{N}}^{\mathrm{H}}$ durch:

$$
G K^{\mathrm{H}}\left(Q_{\mathrm{N}}^{\mathrm{H}}\right)
$$

gekennzeichnet. Dieses Konzept ist für die erstmalige Einführung der im Rahmen der Wirtschaftlichkeitsanalyse verwendeten Größen geeignet, da es die Abhängigkeiten der verschiedenen Größen klar hervorhebt.

Im Hinblick auf die Bewertung von Wärmepumpenkreisläufen im Heiz- und Kühlbetrieb, werden bereits jetzt die folgenden vereinfachten Schreibweisen der Gesamtkosten eingeführt:

\section{- Heizen:}

Gesamtkosten der Bereitstellung einer Nutzwärmemenge $Q_{\mathrm{N}}^{\mathrm{H}}$ $\left(=\right.$ Wärmebereitstellungskosten bei $\left.Q_{\mathrm{N}}^{\mathrm{H}}\right)$ :

$$
G K^{\mathrm{H}}\left(Q_{\mathrm{N}}^{\mathrm{H}}\right)=G K^{\mathrm{H}}
$$

\section{- Kühlen:}

Gesamtkosten der Bereitstellung einer Nutzwärmemenge $Q_{\mathrm{N}}^{\mathrm{K}}$ :

$$
G K^{\mathrm{K}}\left(Q_{\mathrm{N}}^{\mathrm{K}}\right)=G K^{\mathrm{K}}
$$




\section{- Heizen und Kühlen:}

Gesamtkosten der Bereitstellung einer Nutzwärmemenge $Q_{\mathrm{N}}^{\mathrm{H}}+Q_{\mathrm{N}}^{\mathrm{K}}$ :

$$
G K^{\mathrm{H}+\mathrm{K}}\left(Q_{\mathrm{N}}^{\mathrm{H}}+Q_{\mathrm{N}}^{\mathrm{K}}\right)=G K^{\mathrm{H}+\mathrm{K}}
$$

Zur Berechnung der relativen Energiekosteneinsparung im Heizbetrieb werden die Wärmebereitstellungskosten der Wärmepumpe von den Wärmebereitstellungskosten der Vergleichstechnologie abgezogen und die so ermittelte Differenz mit den Wärmebereitstellungskosten der Vergleichstechnologie normiert. Die auf diese Art und Weise gewonnene Kennzahl wird im Folgenden mit $\widehat{E}^{\mathrm{H}}$ angegeben und sei wie folgt definiert:

$$
\widehat{E}^{\mathrm{H}}:=\frac{G K_{\mathrm{kon}}^{\mathrm{H}}-G K_{\mathrm{WP}}^{\mathrm{H}}}{G K_{\mathrm{kon}}^{\mathrm{H}}}
$$

Das primäre Resultat aus Kapitel 3.4.1 in der Nomenklatur von Kapitel 3.4.2 angeschrieben lautet:

$$
\frac{G K_{\mathrm{WP}}^{\mathrm{H}}}{G K_{\mathrm{kon}}^{\mathrm{H}}}=\frac{F_{\mathrm{ges}}^{\mathrm{H}}}{C O P_{\max }^{\mathrm{H}}}<1
$$

Wird der Zusammenhang aus Gleichung (3.77) unter Verwendung von Gleichung (3.78) umgeformt, so ergibt sich für die relative Energiekosteneinsparung im Heizbetrieb:

$$
\begin{aligned}
\widehat{E}^{\mathrm{H}} & =\frac{G K_{\mathrm{kon}}^{\mathrm{H}}-G K_{\mathrm{WP}}^{\mathrm{H}}}{G K_{\mathrm{kon}}^{\mathrm{H}}}=1-\frac{G K_{\mathrm{WP}}^{\mathrm{H}}}{G K_{\mathrm{kon}}^{\mathrm{H}}} \\
\longrightarrow \widehat{E}^{\mathrm{H}} & =1-\frac{F_{\mathrm{ges}}^{\mathrm{H}}}{C O P_{\max }^{\mathrm{H}}}
\end{aligned}
$$

Damit die relative Energiekosteneinsparung im Heizbetrieb zu Null wird, muss nach Gleichung (3.79) gelten, dass $F_{\text {ges }}^{\mathrm{H}}$ gleich dem maximalen COP im Heizbetrieb ist. Dies entspricht dem Resultat aus Gleichung (3.70) zur Ermittlung der Wirtschaftlichkeitsgrenze.

\section{Erstellung von Diagrammen für $\widehat{\boldsymbol{E}}^{\mathbf{H}}$}

Zur graphischen Darstellung von $\widehat{E}^{\mathrm{H}}$ wird der Zusammenhang aus Gleichung (3.79):

$$
\widehat{E}^{\mathrm{H}}=1-\frac{F_{\mathrm{ges}}^{\mathrm{H}}}{C O P_{\max }^{\mathrm{H}}}
$$

im Intervall der Veränderlichen:

$$
\begin{gathered}
0 \leq C O P_{\max }^{\mathrm{H}} \leq 20 \\
0 \leq F_{\text {ges }}^{\mathrm{H}} \leq 20
\end{gathered}
$$

herangezogen. 
Das Ergebnis zeigt Abbildung 3.23:

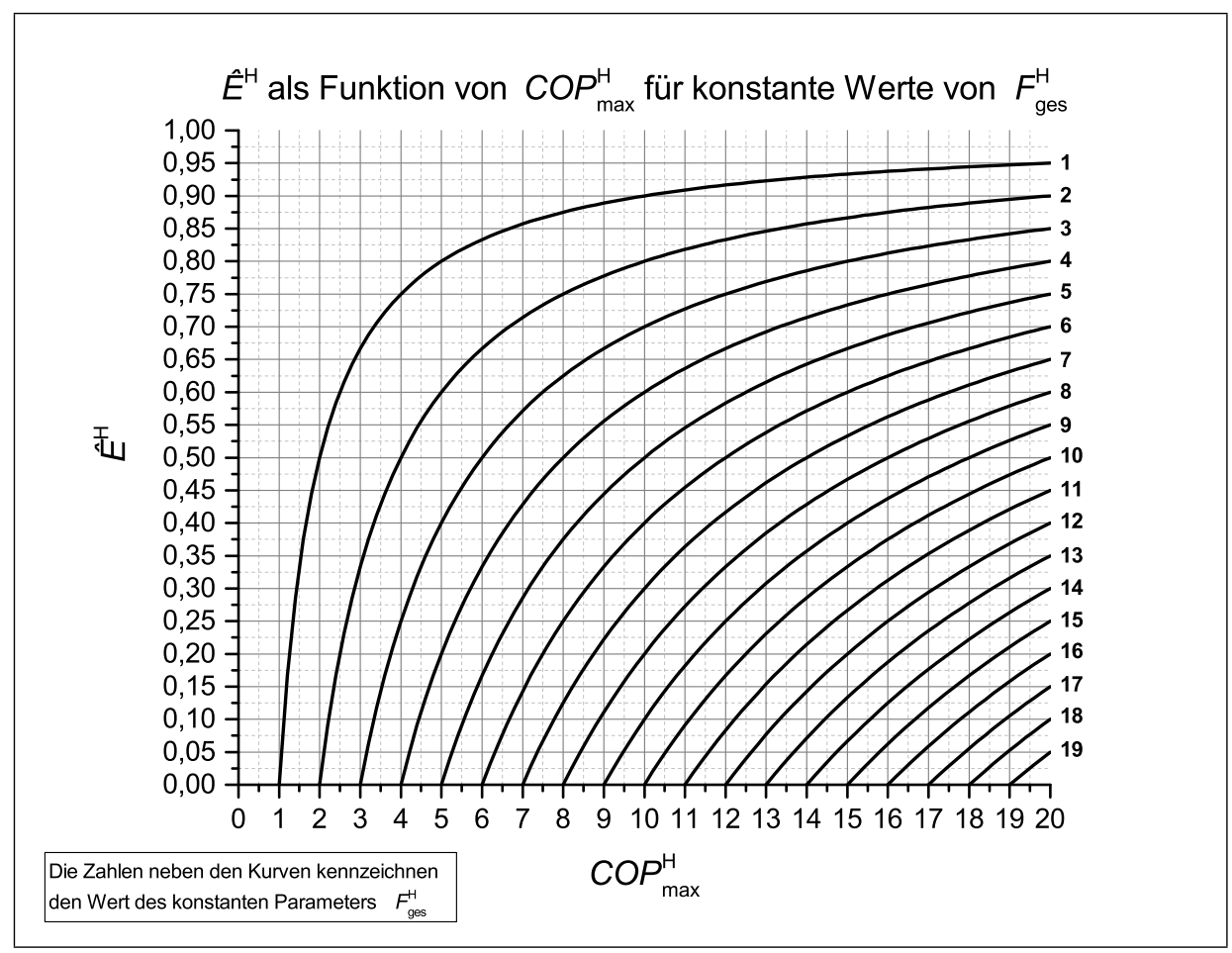

Abbildung 3.23: $\widehat{E}^{\mathrm{H}}$ als Funktion von $C O P_{\max }^{\mathrm{H}}$ für konstante Werte von $F_{\text {ges }}^{\mathrm{H}}$

\section{Hinweise zu den Diagrammen zur Ermittlung von $\widehat{\boldsymbol{E}}^{\mathbf{H}}$}

- Die Abszissenachse in Abbildung 3.23 zeigt $C O P_{\max }^{\mathrm{H}}$. Auf der Ordinate ist $\widehat{E}^{\mathrm{H}}$ aufgetragen.

- Das Intervall der Veränderlichen in Gleichung (3.80) ist aus Abbildung 3.10 sowie Gleichung (3.70) abgeleitet worden.

- Das Gitter in Abbildung 3.23 ist ungewöhnlich fein gewählt worden, um Ablesefehler im Bereich großer Änderungsraten von $\widehat{E}^{\mathrm{H}} \mathrm{zu}$ minimieren.

- Der Ablesevorgang hängt davon ab, ob $\widehat{E}^{\mathrm{H}}$ das Ergebnis aus $C O P_{\max }^{\mathrm{H}}$ und $F_{\text {ges }}^{\mathrm{H}}$ ist oder ob z.B. ein erforderliches $F_{\text {ges }}^{\mathrm{H}}$ für ein gegebenes $\widehat{E}^{\mathrm{H}}$ und einem gegebenen $C O P_{\max }^{\mathrm{H}}$ ermittelt werden soll:

- Aus $C O P_{\text {max }}^{\mathrm{H}}=5$ und $F_{\text {ges }}^{\mathrm{H}}=4$ folgt beispielsweise $\widehat{E}^{\mathrm{H}}=0,2$.

- Um eine relative Energiekosteneinsparung im Heizbetrieb von z.B. mindestens $40 \%$ bei $C O P_{\max }^{\mathrm{H}}=5 \mathrm{zu}$ erreichen, ist ein $F_{\text {ges }}^{\mathrm{H}}$ von maximal 3 erforderlich. 


\subsubsection{Relative Kosteneinsparung im Heiz- und Kühlbetrieb}

Der Heiz- und Kühlbetrieb ist dadurch gekennzeichnet, dass sowohl der vom Arbeitsmedium des Wärmepumpenkreislaufs abgegebene Wärmestrom als auch der vom Arbeitsmedium des Wärmepumpenkreislaufs aufgenommene Wärmestrom als Nutzen einzustufen ist. Dies ist dann der Fall, wenn der Verdampfer des Wärmepumpenkreislaufs so in die Prozesse integriert wird, dass dadurch ein aktiver Kühlbedarf kompensiert bzw. teilkompensiert wird. Ein Beispiel hierfür wäre:

- Zur Kühlung eines Prozessmediums wird über Kältemaschinen bereitgestelltes Kühlwasser verwendet. Der Verdampfer des Wärmepumpenkreislaufs ersetzt die Wärmetauscher des Kühlwassers teilweise oder vollständig.

Zur Berechnung der relativen Energiekosteneinsparung im Heiz- und Kühlbetrieb werden die Gesamtkosten, die beim Heizen und Kühlen mit der Vergleichstechnologie entstehen, von den Gesamtkosten, die beim Heizen und Kühlen mit der Wärmepumpe entstehen, abgezogen und die so ermittelte Differenz mit den Gesamtkosten, die beim Heizen und Kühlen mit der Vergleichstechnologie entstehen, normiert.

Die auf diese Art und Weise gewonnene Kennzahl wird im Folgenden mit $\widehat{E}^{\mathrm{H}+\mathrm{K}}$ angegeben und ist wie folgt definiert:

$$
\widehat{E}^{\mathrm{H}+\mathrm{K}}:=\frac{G K_{\mathrm{kon}}^{\mathrm{H}}+G K_{\mathrm{kon}}^{\mathrm{K}}-G K_{\mathrm{WP}}^{\mathrm{H}+\mathrm{K}}}{G K_{\mathrm{kon}}^{\mathrm{H}}+G K_{\mathrm{kon}}^{\mathrm{K}}}
$$

Das Ziel dieses Kapitels ist es, einen einfachen und leicht zu visualisierenden Zusammenhang für $\widehat{E}^{\mathrm{H}+\mathrm{K}} \mathrm{zu}$ ermitteln. An dieser Stelle sei jedoch zuerst auf eine Besonderheit der relativen Kosteneinsparung im kombinierten Betrieb (= Heiz- und Kühlbetrieb) hingewiesen:

- Bei der Auslegung und Bewertung eines Wärmepumpenkreislaufs im kombinierten Betrieb ist zu berücksichtigen, dass die Kühlleistung in einem festen Verhältnis zur Heizleistung steht. Es gilt der in Gleichung (3.44) ermittelte Zusammenhang:

$$
\widehat{Q}=\frac{\dot{Q}_{\mathrm{N}}^{\mathrm{K}}}{\dot{Q}_{\mathrm{N}}^{\mathrm{H}}}=1-\frac{1}{C O P_{\text {real }}^{\mathrm{H}}}
$$


Der im Verdampfer des Wärmepumpenkreislaufs übertragene Wärmestrom verursacht aus Sicht der Wärmepumpe keine Kosten. Es gilt daher:

$$
G K_{\mathrm{WP}}^{\mathrm{H}+\mathrm{K}}=G K_{\mathrm{WP}}^{\mathrm{H}}
$$

Damit folgt für Gleichung (3.81):

$$
\widehat{E}^{\mathrm{H}+\mathrm{K}}=\frac{G K_{\mathrm{kon}}^{\mathrm{H}}+G K_{\mathrm{kon}}^{\mathrm{K}}-G K_{\mathrm{WP}}^{\mathrm{H}}}{G K_{\mathrm{kon}}^{\mathrm{H}}+G K_{\mathrm{kon}}^{\mathrm{K}}}
$$

Die relative Kosteneinsparung im Heizbetrieb ist nach Gleichung (3.4.3) gegeben durch:

$$
\widehat{E}^{\mathrm{H}}=\frac{G K_{\mathrm{kon}}^{\mathrm{H}}-G K_{\mathrm{WP}}^{\mathrm{H}}}{G K_{\mathrm{kon}}^{\mathrm{H}}}
$$

was umgeformt in Gleichung (3.81) eingesetzt werden kann:

$$
\begin{aligned}
& \widehat{E}^{\mathrm{H}} G K_{\mathrm{kon}}^{\mathrm{H}}=G K_{\mathrm{kon}}^{\mathrm{H}}-G K_{\mathrm{WP}}^{\mathrm{H}} \\
& \longrightarrow \widehat{E}^{\mathrm{H}+\mathrm{K}}=\frac{\widehat{E}^{\mathrm{H}} G K_{\mathrm{kon}}^{\mathrm{H}}+G K_{\mathrm{kon}}^{\mathrm{K}}}{G K_{\mathrm{kon}}^{\mathrm{H}}+G K_{\mathrm{kon}}^{\mathrm{K}}}
\end{aligned}
$$

\section{Einführung des Heizkosten- und des Kühlkostenfaktors}

Es werden eingeführt:

- Heizkostenfaktor $X_{\mathrm{H}}$ :

$$
X_{\mathrm{H}}:=\frac{G K_{\mathrm{kon}}^{\mathrm{H}}}{G K_{\mathrm{kon}}^{\mathrm{H}}+G K_{\mathrm{kon}}^{\mathrm{K}}}
$$

- Kühlkostenfaktor $X_{\mathrm{K}}$ :

$$
X_{\mathrm{K}}:=\frac{G K_{\mathrm{kon}}^{\mathrm{K}}}{G K_{\mathrm{kon}}^{\mathrm{H}}+G K_{\mathrm{kon}}^{\mathrm{K}}}
$$

Anmerkungen zum Heiz- und Kühlkostenfaktor:

Es gilt:

$$
X_{\mathrm{H}}+X_{\mathrm{K}}=1
$$

Daher wäre es mathematisch gesehen nicht notwendig gewesen, zwei Faktoren einzuführen. Im Rahmen der Herleitung eines Zusammenhangs für $\widehat{E}^{\mathrm{H}+\mathrm{K}}$ hat es sich jedoch für die Nachvollziehbarkeit als sinnvoll erwiesen, beide Faktoren einzusetzen und erst am Ende einen der Faktoren zu eliminieren. Da beide Faktoren neben der rechnerischen auch eine reale wirtschaftliche Bedeutung haben, ist es zweckmäßig, beide Faktoren formal im Rechenschema einzuführen. 
Der Heizkostenfaktor beschreibt den relativen Anteil der Heizkosten an den Gesamtkosten des Heizens und des Kühlens unter der Nebenbedingung, dass zwischen der Kühl- und der Heizleistung der Zusammenhang nach Gleichung (3.44) zwingend erfüllt ist:

$$
\widehat{Q}=\frac{\dot{Q}_{\mathrm{N}}^{\mathrm{K}}}{\dot{Q}_{\mathrm{N}}^{\mathrm{H}}}=1-\frac{1}{C O P_{\text {real }}^{\mathrm{H}}}
$$

Steht beispielsweise an der Quelle der Wärmestrom $\dot{Q}_{\mathrm{N}}^{\mathrm{K} 1}$ zur Verfügung, so kann unter Einsatz einer Wärmepumpe auf der Seite der Senke der Wärmestrom:

$$
\dot{Q}_{\mathrm{N} 1}^{\mathrm{H}}=\frac{\dot{Q}_{\mathrm{N}}^{\mathrm{K}}}{\widehat{Q}}=\dot{Q}_{\mathrm{N}}^{\mathrm{K}} \frac{C O P_{\mathrm{real}}^{\mathrm{H}}}{C O P_{\text {real }}^{\mathrm{r}}-1}
$$

eingebunden werden.

Werden die Wärmeströme $\dot{Q}_{\mathrm{N}}^{\mathrm{H} 1}$ und $\dot{Q}_{\mathrm{N}}^{\mathrm{K} 1}$ mit der Vergleichstechnologie bereitgestellt, so entstehen dadurch (über einen gewissen Zeitraum, vgl. Gleichung (3.50)) die Kosten $G K_{\mathrm{kon}}^{\mathrm{H} 1}$ und $G K_{\mathrm{kon}}^{\mathrm{K} 1}$. Der Heizkostenfaktor $X_{\mathrm{H} 1}$ gibt in diesem Fall an, wie hoch der Anteil der Heizkosten $G K_{\text {kon }}^{\mathrm{H} 1}$ an den Gesamtkosten $G K_{\mathrm{kon}}^{\mathrm{H} 1}+G K_{\mathrm{kon}}^{\mathrm{K} 1}$ ist.

Der Heizkostenfaktor $X_{\mathrm{H} 1}$ berücksichtigt dabei aber nicht, ob der Wärmestrom $\dot{Q}_{\mathrm{N}}^{\mathrm{H} 1}$ auf der Seite der Senke im Ausmaß nach Gleichung (3.88) überhaupt benötigt wird.

Das Verständnis dieser Zusammenhänge ist elementar, da zur Ermittlung des Heizkostenund des Kühlkostenfaktors nach Gleichung (3.85) und (3.86) auch wirtschaftliche Zahlen herangezogen werden können. Die Versuchung ist daher groß, an dieser Stelle die Gesamtkosten für das Heizen und das Kühlen mit der Vergleichstechnologie einzusetzen ohne dabei zu berücksichtigen, dass Heiz- und Kühlleistung einer Wärmepumpe nicht entkoppelt voneinander betrachtet werden können. Bleibt dieser Umstand unberücksichtigt, so wird die darauf aufbauende relative Energiekosteneinsparung im Heiz- und Kühlbetrieb in den meisten Fällen nicht korrekt sein.

\section{Verwendung des Heizkosten- und des Kühlkostenfaktors}

Aus Gleichung (3.85) und Gleichung (3.86) folgt:

$$
\begin{aligned}
& X_{\mathrm{H}}=\frac{G K_{\mathrm{kon}}^{\mathrm{H}}}{G K_{\mathrm{kon}}^{\mathrm{H}}+G K_{\mathrm{kon}}^{\mathrm{K}}} \longrightarrow X_{\mathrm{H}}\left(G K_{\mathrm{kon}}^{\mathrm{H}}+G K_{\mathrm{kon}}^{\mathrm{K}}\right)=G K_{\mathrm{kon}}^{\mathrm{H}} \\
& X_{\mathrm{K}}=\frac{G K_{\mathrm{kon}}^{\mathrm{K}}}{G K_{\mathrm{kon}}^{\mathrm{H}}+G K_{\mathrm{kon}}^{\mathrm{K}}} \longrightarrow X_{\mathrm{H}}\left(G K_{\mathrm{kon}}^{\mathrm{H}}+G K_{\mathrm{kon}}^{\mathrm{K}}\right)=G K_{\mathrm{kon}}^{\mathrm{K}}
\end{aligned}
$$

was eingesetzt in Gleichung (3.84) das folgende Zwischenresultat ergibt:

$$
\begin{aligned}
\widehat{E}^{\mathrm{H}+\mathrm{K}} & =\frac{\widehat{E}^{\mathrm{H}} G K_{\mathrm{kon}}^{\mathrm{H}}+G K_{\mathrm{kon}}^{\mathrm{K}}}{G K_{\mathrm{kon}}^{\mathrm{H}}+G K_{\mathrm{kon}}^{\mathrm{K}}} \\
\longrightarrow \widehat{E}^{\mathrm{H}+\mathrm{K}} & =\frac{\widehat{E}^{\mathrm{H}} X_{\mathrm{H}}\left(G K_{\mathrm{kon}}^{\mathrm{H}}+G K_{\mathrm{kon}}^{\mathrm{K}}\right)+X_{\mathrm{H}}\left(G K_{\mathrm{kon}}^{\mathrm{H}}+G K_{\mathrm{kon}}^{\mathrm{K}}\right)}{G K_{\mathrm{kon}}^{\mathrm{H}}+G K_{\mathrm{kon}}^{\mathrm{K}}}
\end{aligned}
$$


Daraus folgt der gesuchte Zusammenhang für die relative Energiekosteneinsparung im kombinierten Betrieb:

$$
\widehat{E}^{\mathrm{H}+\mathrm{K}}=\widehat{E}^{\mathrm{H}} X_{\mathrm{H}}+X_{\mathrm{K}}
$$

Das Resultat aus Gleichung (3.91) kann wie folgt gedeutet werden:

- Die relative Energiekosteneinsparung im kombinierten Betrieb ergibt sich dadurch, dass die relative Energiekosteneinsparung im Heizbetrieb mit dem Heizkostenfaktor multipliziert wird und dem daraus resultierenden Produkt der Kühlkostenfaktor hinzu addiert wird.

- Wenn nicht gekühlt wird bzw. für das Kühlen keine Kosten entstehen, so gilt $X_{\mathrm{H}}=1$ und $X_{\mathrm{K}}=0$. Dann ist die relative Energiekosteneinsparung im kombinierten Betrieb gleich der im Heizbetrieb. Dies entspricht der Erwartung.

Unter Berücksichtigung von Gleichung (3.87), lässt sich aus Gleichung (3.91) einer der Faktoren eliminieren:

$$
\begin{aligned}
& \widehat{E}^{\mathrm{H}+\mathrm{K}}=\widehat{E}^{\mathrm{H}}+X_{\mathrm{K}}\left(1-\widehat{E}^{\mathrm{H}}\right) \\
& \widehat{E}^{\mathrm{H}+\mathrm{K}}=1+X_{\mathrm{H}}\left(\widehat{E}^{\mathrm{H}}-1\right)
\end{aligned}
$$

Für die spätere Visualisierung $\widehat{E}^{\mathrm{H}+\mathrm{K}}$ ist diese Erkenntnis insofern relevant, da $\widehat{E}^{\mathrm{H}+\mathrm{K}}$ als Funktion von $\widehat{E}^{\mathrm{H}}$ und $X_{\mathrm{H}}$ bzw. $\widehat{E}^{\mathrm{H}}$ und $X_{\mathrm{K}}$ dargestellt werden kann.

\section{Bestimmung eines Zusammenhangs für $\boldsymbol{X}_{\mathbf{H}}$ und $\boldsymbol{X}_{\mathbf{K}}$}

Zur Bestimmung eines Zusammenhangs für $X_{\mathrm{H}}$ werden an der Definition aus Gleichung (3.85) die folgenden Umformschritte vollzogen:

$$
\begin{aligned}
& X_{\mathrm{H}}=\frac{G K_{\mathrm{kon}}^{\mathrm{H}}}{\mathrm{GK}_{\mathrm{kon}}^{\mathrm{H}}+G K_{\mathrm{kon}}^{\mathrm{K}}} \\
& X_{\mathrm{H}}=\frac{G K_{\mathrm{WP}}^{\mathrm{H}}\left(\frac{G K_{\text {kon }}^{\mathrm{H}}}{G K_{\mathrm{WP}}^{\mathrm{H}}}\right)}{G K_{\mathrm{WP}}^{\mathrm{H}}\left(\frac{G K_{\mathrm{kon}}^{\mathrm{H}}}{G K_{\mathrm{WP}}^{\mathrm{H}}}+\frac{G K_{\mathrm{kon}}^{\mathrm{K}}}{G K_{\mathrm{WP}}^{\mathrm{H}}}\right)} \\
& X_{\mathrm{H}}=\frac{\frac{G K_{\mathrm{kon}}^{\mathrm{H}}}{G K_{\mathrm{WP}}^{\mathrm{H}}}}{\frac{G K_{\mathrm{kon}}^{\mathrm{H}}}{G K_{\mathrm{WP}}^{\mathrm{H}}}+\frac{G K_{\mathrm{kon}}^{\mathrm{K}}}{G K_{\mathrm{WP}}^{\mathrm{H}}}}
\end{aligned}
$$


Der Quotient aus den Wärmebereitstellungskosten der Vergleichstechnologie und den Wärmebereitstellungskosten der Wärmepumpe ist nach Gleichung (3.78) gegeben durch:

$$
\frac{G K_{\mathrm{WP}}^{\mathrm{H}}}{G K_{\mathrm{kon}}^{\mathrm{H}}}=\frac{F_{\mathrm{ges}}^{\mathrm{H}}}{C O P_{\max }^{\mathrm{H}}} \longrightarrow \frac{G K_{\mathrm{kon}}^{\mathrm{H}}}{G K_{\mathrm{WP}}^{\mathrm{H}}}=\frac{C O P_{\max }^{\mathrm{H}}}{F_{\text {ges }}^{\mathrm{H}}}
$$

was in Gleichung (3.93) eingesetzt die folgenden Umformungen erlaubt:

$$
\begin{aligned}
X_{\mathrm{H}}= & \frac{\frac{G K_{\text {kon }}^{\mathrm{H}}}{G K_{\mathrm{WP}}^{\mathrm{H}}}}{\frac{G K_{\mathrm{kon}}^{\mathrm{H}}}{G K_{\mathrm{WP}}^{\mathrm{H}}}+\frac{G K_{\mathrm{kon}}^{\mathrm{K}}}{G K_{\mathrm{WP}}^{\mathrm{H}}}} \\
\longrightarrow X_{\mathrm{H}}= & \frac{\frac{C O P_{\text {max }}^{\mathrm{H}}}{F_{\mathrm{ges}}^{\mathrm{H}}}}{\frac{C O P_{\text {max }}^{\mathrm{H}}}{F_{\mathrm{ges}}^{\mathrm{H}}}+\frac{G K_{\mathrm{kon}}^{\mathrm{K}}}{G K_{\mathrm{WP}}^{\mathrm{H}}}}=\frac{1}{1+\frac{F_{\mathrm{ges}}^{\mathrm{H}}}{C O P_{\mathrm{max}}^{\mathrm{H}}} \frac{G K_{\mathrm{kon}}^{\mathrm{K}}}{G K_{\mathrm{WP}}^{\mathrm{H}}}}
\end{aligned}
$$

Für die Wärmebereitstellungskosten der Wärmepumpe im Heizbetrieb gilt nach Gleichung (3.54):

$$
G K_{\mathrm{WP}}^{\mathrm{H}}=\frac{Q_{\mathrm{N}}^{\mathrm{H}} K_{\mathrm{WP}}^{\mathrm{H}}}{\eta_{\mathrm{WP}} C O P_{\max }^{\mathrm{H}}}
$$

Die Gesamtkosten, die beim Kühlen mit der Vergleichstechnologie entstehen, lassen sich in Analogie zu den Wärmebereitstellungskosten der Vergleichstechnologie wie folgt darstellen:

$$
G K_{\mathrm{kon}}^{\mathrm{K}}=\frac{Q_{\mathrm{N}}^{\mathrm{K}}}{\eta_{\mathrm{kon}}^{\mathrm{K}}} K_{\mathrm{kon}}^{\mathrm{K}}
$$

Werden die Resultate aus Gleichung (3.54) und Gleichung (3.95) in Gleichung (3.94) eingesetzt, so folgt für den Heizkostenfaktor:

$$
\begin{aligned}
X_{\mathrm{H}} & =\frac{1}{1+\frac{F_{\mathrm{ges}}^{\mathrm{H}}}{C O P_{\text {max }}^{\mathrm{H}}} \frac{G K_{\mathrm{kon}}^{\mathrm{K}}}{G K_{\mathrm{WP}}^{\mathrm{H}}}} \\
\longrightarrow X_{\mathrm{H}} & =\frac{1}{1+\frac{F_{\text {ges }}^{\mathrm{H}}}{C O P_{\text {max }}^{\mathrm{H}}} \frac{Q_{\mathrm{N}}^{\mathrm{K}}}{Q_{\mathrm{N}}^{\mathrm{H}}} \frac{K_{\mathrm{kon}}^{\mathrm{K}}}{K_{\mathrm{WP}}^{\mathrm{H}}} \frac{\eta_{\mathrm{WP}}}{\eta_{\mathrm{kon}}^{\mathrm{K}}} \frac{C O P_{\text {max }}^{\mathrm{H}}}{1}} \\
X_{\mathrm{H}} & =\frac{1}{1+\widehat{Q} \frac{F_{\text {ges }}^{\mathrm{H}}}{1} \frac{K_{\mathrm{kon}}^{\mathrm{K}}}{K_{\mathrm{WP}}^{\mathrm{H}}} \frac{\eta_{\mathrm{WP}}}{\eta_{\mathrm{kon}}^{\mathrm{K}}}}
\end{aligned}
$$

Der Gesamteinflussfaktor im Heizbetrieb ist nach Gleichung (3.66) gegeben durch:

$$
F_{\text {ges }}^{\mathrm{H}}=\frac{K_{\mathrm{WP}}^{\mathrm{H}}}{K_{\mathrm{kon}}^{\mathrm{H}}} \frac{\eta_{\mathrm{kon}}^{\mathrm{H}}}{\eta_{\mathrm{WP}}}
$$


Wird er in Gleichung (3.96) eingesetzt, so ergibt sich:

$$
\begin{aligned}
X_{\mathrm{H}} & =\frac{1}{1+\widehat{Q} \frac{F_{\mathrm{ges}}^{\mathrm{H}}}{1} \frac{K_{\mathrm{kon}}^{\mathrm{K}}}{K_{\mathrm{WP}}^{\mathrm{H}}} \frac{\eta_{\mathrm{WP}}}{\eta_{\mathrm{kon}}^{\mathrm{K}}}} \\
\longrightarrow X_{\mathrm{H}} & =\frac{1}{1+\widehat{Q} \frac{K_{\mathrm{WP}}^{\mathrm{H}}}{K_{\mathrm{kon}}^{\mathrm{H}}} \frac{\eta_{\mathrm{kon}}^{\mathrm{H}}}{\eta_{\mathrm{WP}}} \frac{K_{\mathrm{kon}}^{\mathrm{K}}}{K_{\mathrm{WP}}^{\mathrm{H}}} \frac{\eta_{\mathrm{WP}}}{\eta_{\mathrm{kon}}^{\mathrm{K}}}} \\
X_{\mathrm{H}} & =\frac{1}{1+\widehat{Q} \frac{\eta_{\mathrm{kon}}^{\mathrm{H}}}{\eta_{\mathrm{kon}}^{\mathrm{K}}} \frac{K_{\mathrm{kon}}^{\mathrm{K}}}{K_{\mathrm{kon}}^{\mathrm{H}}}}
\end{aligned}
$$

Zur Ermittlung von:

$$
\frac{\eta_{\mathrm{kon}}^{\mathrm{H}}}{\eta_{\mathrm{kon}}^{\mathrm{K}}} \frac{K_{\mathrm{kon}}^{\mathrm{K}}}{K_{\mathrm{kon}}^{\mathrm{H}}}
$$

kann der in Gleichung (3.68) definierte Gesamteinflussfaktor im Heiz- und Kühlbetrieb herangezogen werden:

$$
F_{\mathrm{ges}}^{\mathrm{H}+\mathrm{K}}=\frac{K_{\mathrm{kon}}^{\mathrm{K}}}{K_{\mathrm{kon}}^{\mathrm{H}}} \frac{\eta_{\mathrm{kon}}^{\mathrm{H}}}{\eta_{\mathrm{kon}}^{\mathrm{K}}}
$$

Mit dem Gesamteinflussfaktor im Heiz- und Kühlbetrieb folgt für den Heizkostenfaktor $X_{\mathrm{H}}$ :

$$
\begin{aligned}
X_{\mathrm{H}} & =\frac{1}{1+\widehat{Q} \frac{\eta_{\mathrm{kon}}^{\mathrm{H}}}{\eta_{\mathrm{kon}}^{\mathrm{K}}} \frac{K_{\mathrm{kon}}^{\mathrm{K}}}{K_{\mathrm{kon}}^{\mathrm{H}}}} \\
\longrightarrow X_{\mathrm{H}} & =\frac{1}{1+\widehat{Q} F_{\mathrm{ges}}^{\mathrm{H}+\mathrm{K}}}
\end{aligned}
$$

Unter Berücksichtigung, dass die Summe aus Heiz- und Kühlkostenfaktor 1 ergeben muss, lässt sich in wenigen Umformschritten die Bestimmungsgleichung für den Kühlkostenfaktor ermitteln:

$$
\begin{aligned}
& X_{\mathrm{H}}=\frac{1}{1+\widehat{Q} F_{\mathrm{ges}}^{\mathrm{H}+\mathrm{K}}}=1-X_{\mathrm{K}} \\
& X_{\mathrm{K}}=\frac{1}{1+\frac{1}{\widehat{Q}} \frac{1}{F_{\mathrm{ges}}^{\mathrm{H}+\mathrm{K}}}}=1-X_{\mathrm{H}}
\end{aligned}
$$




\section{Hinweise zur Ermittlung von $\boldsymbol{F}_{\text {ges }}^{\mathbf{H}+\mathrm{K}}$}

Der Gesamteinflussfaktor im Heiz- und Kühlbetrieb ist nach Gleichung (3.68) gegeben durch:

$$
\begin{aligned}
F_{\mathrm{ges}}^{\mathrm{H}+\mathrm{K}} & =\frac{F_{\mathrm{ges}}^{\mathrm{H}}}{F_{\mathrm{ges}}^{\mathrm{K}}} \\
\longrightarrow F_{\mathrm{ges}}^{\mathrm{H}+\mathrm{K}} & =\frac{K_{\mathrm{kon}}^{\mathrm{K}}}{K_{\mathrm{kon}}^{\mathrm{H}}} \frac{\eta_{\mathrm{kon}}^{\mathrm{H}}}{\eta_{\mathrm{kon}}^{\mathrm{K}}}
\end{aligned}
$$

Im Bezug auf den Gesamteinflussfaktor im Kühlbetrieb sind, wie bereits in Kapitel 3.4.1 bei den Ausführungen zum Intervall von $F_{\mathrm{B}}^{\mathrm{A}}$ angemerkt, zwei Fälle zu unterscheiden:

\section{Fall 1:}

Die Vergleichstechnologie mit der gekühlt wird, entspricht einer Kaltdampf-Kompressions-Kälteanlage mit bekanntem $C O P_{\text {real }}^{\mathrm{Kv}}$.

\section{Fall 2:}

Die Vergleichstechnologie mit der gekühlt wird, entspricht keiner Kaltdampf-Kompressions-Kälteanlage mit bekanntem $C O P_{\text {real }}^{\mathrm{Kv}}$. Alles was nicht unter Fall 1 fällt ist daher Fall 2 zuzuordnen.

Für beide Fälle ist zu ermitteln, wie die Komponenten von $F_{\text {ges }}^{\mathrm{H}+\mathrm{K}}$ berechnet werden und ob die in Kapitel 3.4.1 getätigte Annahme von $F_{\mathrm{B}}^{\mathrm{A}} \leq 20$ erfüllt ist. Falls ja, dann ist es möglich, sowohl $F_{\text {ges }}^{\mathrm{H}}$ als auch $F_{\text {ges }}^{\mathrm{H}+\mathrm{K}}$ aus einem Diagramm vom Typ $F_{\mathrm{B}}^{\mathrm{A}}$ abzulesen, wobei das Diagramm dann nur das Intervall $0 \leq F_{\mathrm{B}}^{\mathrm{A}} \leq 20$ darstellen müsste.

Fall 1 | Komponenten von $F_{\text {ges }}^{\mathrm{H}+\mathrm{K}}$

Stellt eine Kälteanlage mit bekanntem $C O P_{\text {real }}^{\mathrm{Kv}}$ die Vergleichstechnologie dar (vgl. Kapitel 3.3.6), so lassen sich die mit der Kühlung verbundenen Kosten wie folgt abbilden:

$$
G K_{\mathrm{kon}}^{\mathrm{K}}=W_{\mathrm{kon}}^{\mathrm{K}} K_{\mathrm{el}}=Q_{\mathrm{N}}^{\mathrm{K}} K_{\mathrm{kon}}^{\mathrm{K}}
$$

Daraus folgt mit der Definition von $C O P_{\text {real }}^{\mathrm{Kv}}$ in Gleichung (3.46):

$$
\begin{aligned}
K_{\mathrm{kon}}^{\mathrm{K}} & =\frac{W_{\mathrm{kon}}^{\mathrm{K}}}{Q_{\mathrm{N}}^{\mathrm{K}}} K_{\mathrm{el}} \\
\longrightarrow K_{\mathrm{kon}}^{\mathrm{K}} & =\frac{K_{\mathrm{el}}}{C O P_{\mathrm{real}}^{\mathrm{Kv}}}
\end{aligned}
$$

Bei der Herleitung der spezifischen Kühlkosten der Vergleichstechnologie nach Gleichung (3.102) ist stillschweigend vorausgesetzt worden, dass bei Berücksichtigung von $C O P_{\text {real }}^{\mathrm{Kv}}$ der Konversionswirkungsgrad $\eta_{\mathrm{kon}}^{\mathrm{K}} \mathrm{zu} 1$ wird. Ob ggf. ein anderer Ansatz, der ohne diese Annahme auskommt, günstiger wäre, wird in Kapitel 6.3 aufgegriffen. 
Aus den Ausführungen folgt für Fall 1:

- Bei der Ermittlung von:

$$
F_{\mathrm{ges}}^{\mathrm{H}+\mathrm{K}}=\frac{K_{\mathrm{kon}}^{\mathrm{K}}}{K_{\mathrm{kon}}^{\mathrm{H}}} \frac{\eta_{\mathrm{kon}}^{\mathrm{H}}}{\eta_{\mathrm{kon}}^{\mathrm{K}}}
$$

wird:

$$
\eta_{\mathrm{kon}}^{\mathrm{K}}=1 \quad K_{\mathrm{kon}}^{\mathrm{K}}=\frac{K_{\mathrm{el}}}{C O P_{\mathrm{real}}^{\mathrm{Kv}}}
$$

gesetzt.

Fall $1 \mid$ Gültigkeit der Annahme $F_{\mathrm{B}}^{\mathrm{A}} \leq 20$

Die in Kapitel 3.4.1 angeführte Bedingung, um die obere Grenze von $F_{\text {ges }}^{\mathrm{H}}$ auch für $F_{\mathrm{B}}^{\mathrm{A}} \mathrm{zu}$ verwenden, ist nach Gleichung (3.71) gegeben durch:

$$
F_{\text {ges }}^{\mathrm{K}}>1
$$

was unter Verwendung der Definition des Gesamteinflussfaktors im Kühlbetrieb nach Gleichung (3.67):

$$
F_{\mathrm{ges}}^{\mathrm{K}}=\frac{K_{\mathrm{WP}}^{\mathrm{K}}}{K_{\mathrm{kon}}^{\mathrm{K}}} \frac{\eta_{\mathrm{kon}}^{\mathrm{K}}}{\eta_{\mathrm{WP}}}
$$

in folgende Bedingung überführt werden kann:

$$
\frac{K_{\mathrm{WP}}^{\mathrm{K}}}{K_{\mathrm{kon}}^{\mathrm{K}}} \frac{\eta_{\mathrm{kon}}^{\mathrm{K}}}{\eta_{\mathrm{WP}}}>1
$$

Das Resultat aus Gleichung (3.102) in Gleichung (3.104) eingesetzt, unter Berücksichtigung von:

$$
\begin{gathered}
K_{\mathrm{WP}}^{\mathrm{K}}=K_{\mathrm{WP}}^{\mathrm{H}}=K_{\mathrm{el}} \\
\eta_{\mathrm{kon}}^{\mathrm{K}}=1
\end{gathered}
$$

ergibt:

$$
\begin{aligned}
\frac{K_{\mathrm{WP}}^{\mathrm{K}}}{K_{\mathrm{kon}}^{\mathrm{K}}} \frac{\eta_{\mathrm{kon}}^{\mathrm{K}}}{\eta_{\mathrm{WP}}}>1 \\
\longrightarrow \frac{C O P_{\mathrm{real}}^{\mathrm{Kv}}}{\eta_{\mathrm{WP}}}>1
\end{aligned}
$$

Was schlussendlich zur der folgenden Bedingung für $F_{\text {ges }}^{\mathrm{H}}>F_{\text {ges }}^{\mathrm{K}}$ führt:

$$
C O P_{\text {real }}^{\mathrm{Kv}}>\eta_{\mathrm{WP}}
$$

Ohne Beweis kann unterstellt werden, dass die Bedingung nach Gleichung (3.107) für fast alle Kaltdampf-Kompressions-Kälteanlage erfüllt sein wird. 
Daraus folgt für Fall 1:

- Es kann $F_{\mathrm{B}}^{\mathrm{A}} \leq 20$ angenommen werden.

Fall $2 \mid$ Komponenten von $F_{\text {ges }}^{\mathrm{H}+\mathrm{K}}$

Die Kosten, die im Rahmen der Kühlung mit der Vergleichstechnologie bei Fall 2 anfallen, lassen sich auf ähnliche Weise wie die Wärmebereitstellungskosten der Vergleichstechnologie darstellen:

$$
G K_{\mathrm{kon}}^{\mathrm{K}}=Q_{\mathrm{kon}}^{\mathrm{K}} K_{\mathrm{kon}}^{\mathrm{K}}=\frac{Q_{\mathrm{N}}^{\mathrm{K}}}{\eta_{\mathrm{kon}}^{\mathrm{K}}} K_{\mathrm{kon}}^{\mathrm{K}}
$$

Daraus folgt für Fall 2:

- Bei der Ermittlung von:

$$
F_{\mathrm{ges}}^{\mathrm{H}+\mathrm{K}}=\frac{K_{\mathrm{kon}}^{\mathrm{K}}}{K_{\mathrm{kon}}^{\mathrm{H}}} \frac{\eta_{\mathrm{kon}}^{\mathrm{H}}}{\eta_{\mathrm{kon}}^{\mathrm{K}}}
$$

sind die Größen $\eta_{\mathrm{kon}}^{\mathrm{K}}$ und $K_{\mathrm{kon}}^{\mathrm{K}}$ passend zur realen betrieblichen Situation zu wählen.

Fall $2 \mid$ Gültigkeit der Annahme $F_{\mathrm{B}}^{\mathrm{A}} \leq 20$

Der Nachweis der Gültigkeit von $F_{\mathrm{B}}^{\mathrm{A}} \leq 20$ wird in Fall 2 nicht auf Basis des Gesamteinflussfaktors im Kühlbetrieb geführt. Vielmehr wird direkt ermittelt, welche Konstellationen:

$$
F_{\mathrm{ges}}^{\mathrm{H}+\mathrm{K}}=\frac{K_{\mathrm{kon}}^{\mathrm{K}}}{K_{\mathrm{kon}}^{\mathrm{H}}} \frac{\eta_{\mathrm{kon}}^{\mathrm{H}}}{\eta_{\mathrm{kon}}^{\mathrm{K}}}<20
$$

erfüllen und ob diese Konstellationen auch die realen betrieblichen Bedingungen abbilden.

Aus Gleichung (3.109) folgt:

$$
\frac{K_{\mathrm{kon}}^{\mathrm{K}}}{K_{\mathrm{kon}}^{\mathrm{H}}}<20 \frac{\eta_{\mathrm{kon}}^{\mathrm{K}}}{\eta_{\mathrm{kon}}^{\mathrm{H}}}
$$

Für eine grobe Abschätzung wird unterstellt, dass $\eta_{\mathrm{kon}}^{\mathrm{H}} \sim \eta_{\mathrm{kon}}^{\mathrm{K}}$ gesetzt werden kann. Somit ergibt sich für die Bedingung des Gesamteinflussfaktors im Heiz- und Kühlbetrieb nach Gleichung (3.109):

$$
\frac{K_{\text {kon }}^{\mathrm{K}}}{K_{\mathrm{kon}}^{\mathrm{H}}}<20
$$

Daraus folgt, dass $F_{\text {ges }}^{\mathrm{H}+\mathrm{K}}$ kleiner als 20 ist, wenn $\eta_{\mathrm{kon}}^{\mathrm{H}} \sim \eta_{\mathrm{kon}}^{\mathrm{K}}$ gesetzt werden kann und die spezifischen Kosten im Kühlbetrieb nicht mehr als das 20-fache der spezifischen Kosten im Heizbetrieb betragen.

Ersetzt eine Wärmepumpe eine Kältemaschine (mit unbekanntem $C O P_{\text {real }}^{\mathrm{Kv}}$ ), so kann unterstellt werden, dass Gleichung (3.109) erfüllt ist. Ersetzt eine Wärmepumpe keine Kältemaschine, wie es beispielsweise bei einem luftgekühlten Kondensator der Fall wäre, so gehen in die spezifischen Kühlkosten $K_{\mathrm{kon}}^{\mathrm{K}}$ nur die spezifischen Kosten der elektrischen Energie 
der Ventilatoren ein. Es darf daher angenommen werden, dass die spezifischen Kühlkosten in diesem Fall zumindest nicht größer sein werden als es bei der Kühlung mit einer Kältemaschine der Fall wäre, da ansonsten aus wirtschaftlichen Gründen eine Kältemaschine eingesetzt würde.

Unter der Annahme, dass Gleichung (3.109) für eine Kältemaschine als erfüllt angesehen werden kann, und der Bedingung, dass bei der Kühlung ohne Kältemaschine zumindest keine höheren spezifischen Kühlkosten entstehen als bei der Kühlung mit einer Kältemaschine, folgt für Fall 2:

- Es kann $F_{\mathrm{B}}^{\mathrm{A}} \leq 20$ gesetzt werden.

Hinweis: Sollten sich die für Fall 2 getätigten Annahmen als grob falsch erweisen, so kann das Bewertungsschema trotzdem verwendet werden. Einzig die Möglichkeit, $F_{\text {ges }}^{\mathrm{H}+\mathrm{K}}$ aus den Diagrammen für $F_{\mathrm{B}}^{\mathrm{A}}$ abzulesen, wäre dann nicht gegeben.

\section{Ermittlung von $\boldsymbol{F}_{\text {ges }}^{\mathrm{H}+\mathrm{K}}$ aus den Diagrammen von $\boldsymbol{F}_{\mathrm{B}}^{\mathrm{A}}$}

Um $F_{\text {ges }}^{\mathrm{H}+\mathrm{K}}$ aus den Diagrammen für $F_{\mathrm{B}}^{\mathrm{A}}$ ablesen zu können, sind zuerst die für die Ablesung notwendigen Größen zu ermitteln. Es gilt:

$$
\begin{gathered}
F_{\mathrm{B}}^{\mathrm{A}}=\frac{K_{\mathrm{A}}}{K_{\mathrm{B}}} \frac{\eta_{\mathrm{B}}}{\eta_{\mathrm{A}}} \\
F_{\text {ges }}^{\mathrm{H}+\mathrm{K}}=\frac{K_{\mathrm{kon}}^{\mathrm{K}}}{K_{\mathrm{kon}}^{\mathrm{H}}} \frac{\eta_{\mathrm{kon}}^{\mathrm{H}}}{\eta_{\mathrm{kon}}^{\mathrm{K}}}
\end{gathered}
$$

Wobei für den Ableseprozess zu setzen ist:

\section{Fall 1:}

$$
\begin{array}{ll}
\eta_{\mathrm{A}}=\eta_{\mathrm{kon}}^{\mathrm{K}}=1 & \eta_{\mathrm{B}}=\eta_{\mathrm{kon}}^{\mathrm{H}} \\
K_{\mathrm{A}}=K_{\mathrm{kon}}^{\mathrm{H}} & K_{\mathrm{B}}=K_{\mathrm{kon}}^{\mathrm{K}}=\frac{K_{\mathrm{el}}}{C O P_{\text {real }}^{\mathrm{Kv}}}
\end{array}
$$

Fall 2:

$$
\begin{aligned}
\eta_{\mathrm{A}} & =\eta_{\mathrm{kon}}^{\mathrm{K}} & \eta_{\mathrm{B}} & =\eta_{\mathrm{kon}}^{\mathrm{H}} \\
K_{\mathrm{A}} & =K_{\mathrm{kon}}^{\mathrm{H}} & K_{\mathrm{B}} & =K_{\mathrm{kon}}^{\mathrm{K}}
\end{aligned}
$$

Unter Verwendung der in Gleichung (3.112) und Gleichung (3.113) angegebenen Zusammenhänge wird in einem ersten Schritt der Quotient aus $\eta_{\mathrm{kon}}^{\mathrm{H}}$ und $\eta_{\mathrm{kon}}^{\mathrm{K}}$ aus Abbildung 3.19 bzw. 3.20 ermittelt. Im Anschluss kann der gesuchte Wert für $F_{\text {ges }}^{\mathrm{H}+\mathrm{K}}$ aus Abbildung 3.21 abgelesen werden. 


\section{Erstellung von Diagrammen für $\boldsymbol{X}_{\mathbf{H}}$ und $\boldsymbol{X}_{\mathbf{H}}$}

Die Zusammenhänge zur Bestimmung von $X_{\mathrm{H}}$ und $X_{\mathrm{K}}$ sind nach Gleichung (3.100) gegeben durch:

$$
\begin{aligned}
& X_{\mathrm{H}}=\frac{1}{1+\widehat{Q} F_{\text {ges }}^{\mathrm{H}+\mathrm{K}}}=1-X_{\mathrm{K}} \\
& X_{\mathrm{K}}=\frac{1}{1+\frac{1}{\widehat{Q}} \frac{1}{F_{\text {ges }}^{\mathrm{H}+\mathrm{K}}}}=1-X_{\mathrm{H}}
\end{aligned}
$$

Für die darzustellenden Intervalle gilt:

$$
\begin{gathered}
0 \leq \widehat{Q} \leq 1 \\
0 \leq F_{\text {ges }}^{\mathrm{H}+\mathrm{K}} \leq 20
\end{gathered}
$$

Abbildung 3.24 und 3.25 zeigen den Heizkostenfaktor $X_{\mathrm{H}}$, Abbildung 3.26 und 3.27 den Kühlkostenfaktor $X_{\mathrm{K}}$, jeweils als Funktion von $F_{\text {ges }}^{\mathrm{H}+\mathrm{K}}$ für konstante Werte von $\widehat{Q}$.

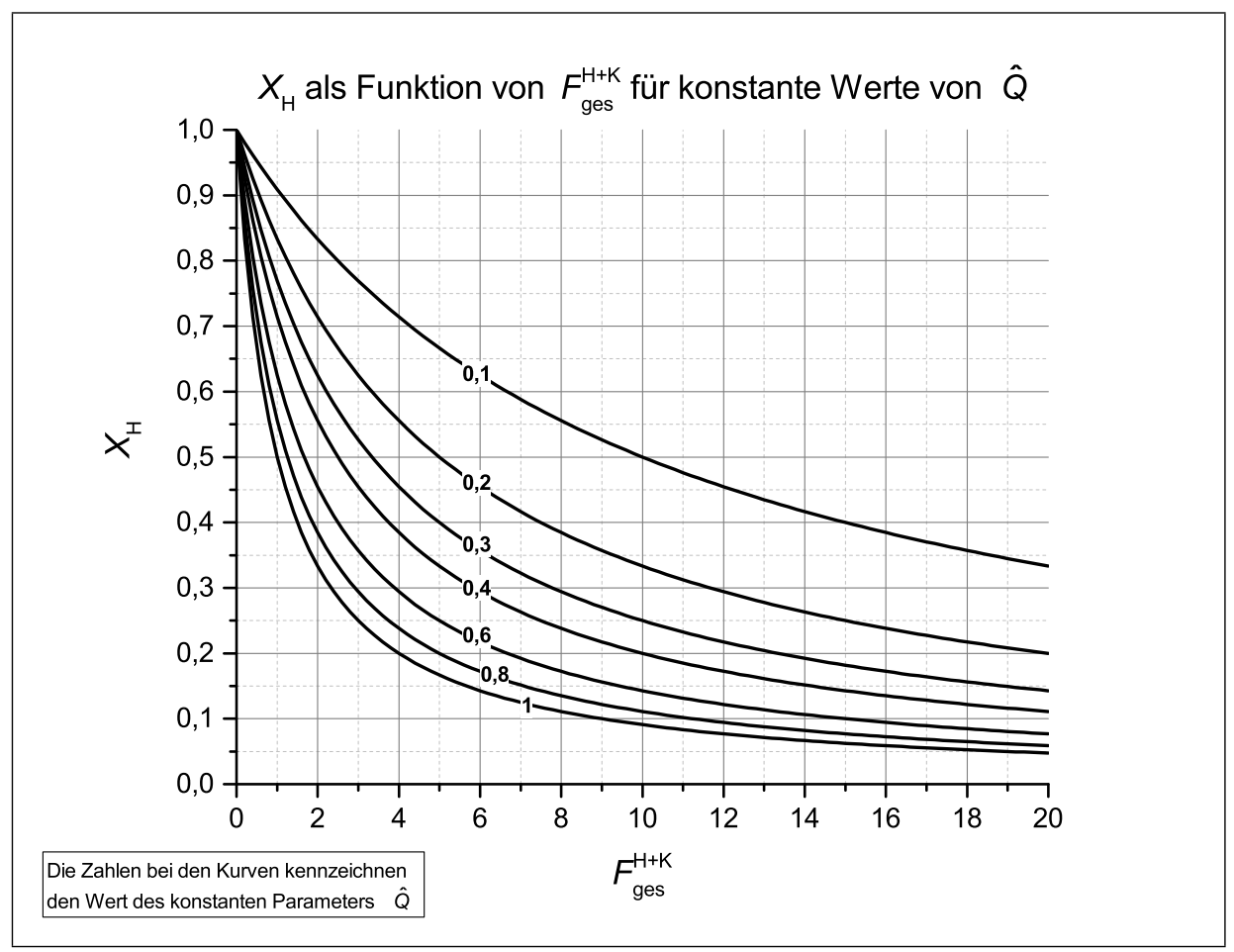

Abbildung 3.24: $X_{\mathrm{H}}$ als Funktion von $F_{\text {ges }}^{\mathrm{H}+\mathrm{K}}$ für konstante Werte von $\widehat{Q}$ 


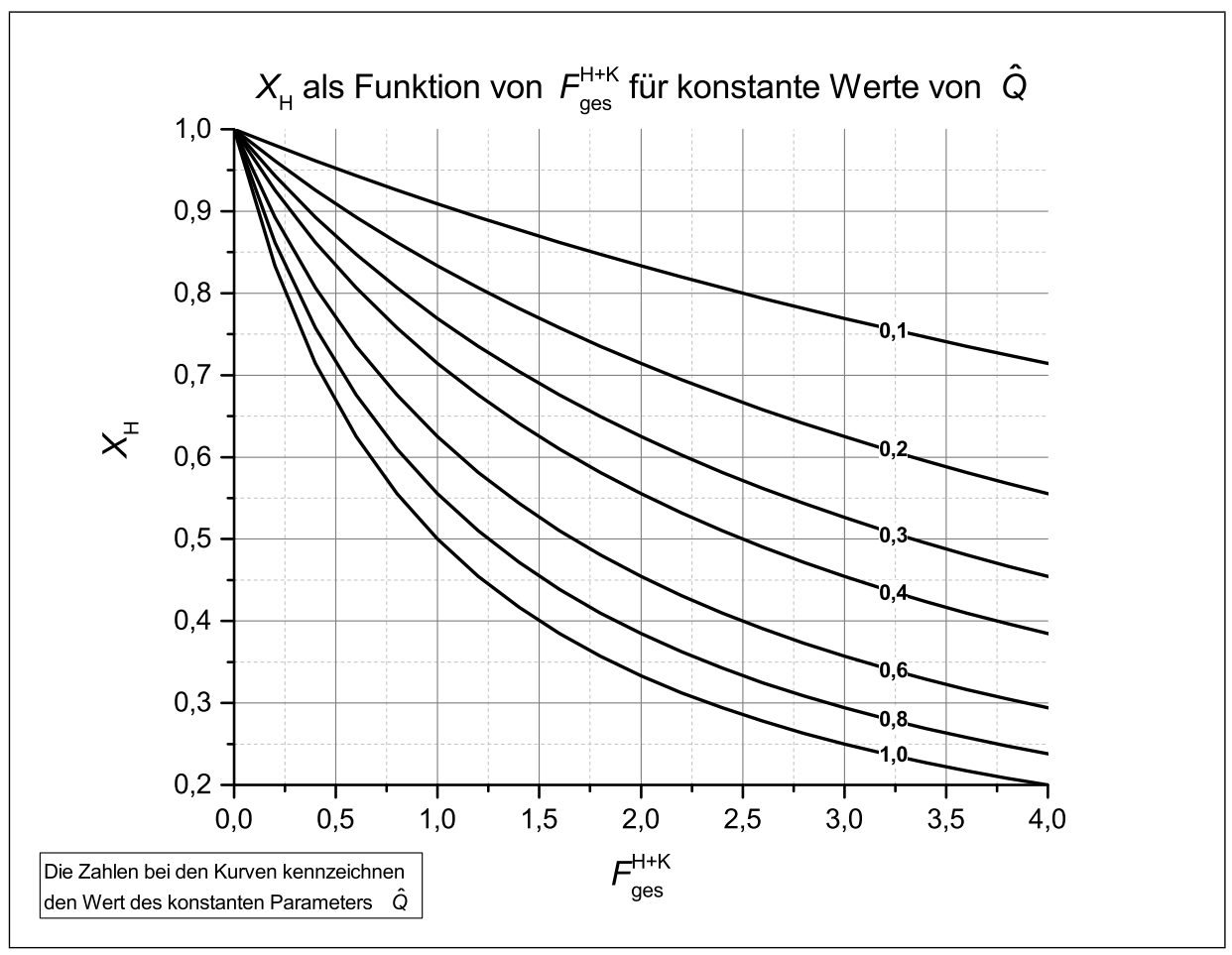

Abbildung 3.25: $X_{\mathrm{H}}$ als Funktion von $F_{\text {ges }}^{\mathrm{H}+\mathrm{K}}$ im Intervall $0 \leq F_{\text {ges }}^{\mathrm{H}+\mathrm{K}} \leq 4$ für konstante Werte von $\widehat{Q}$

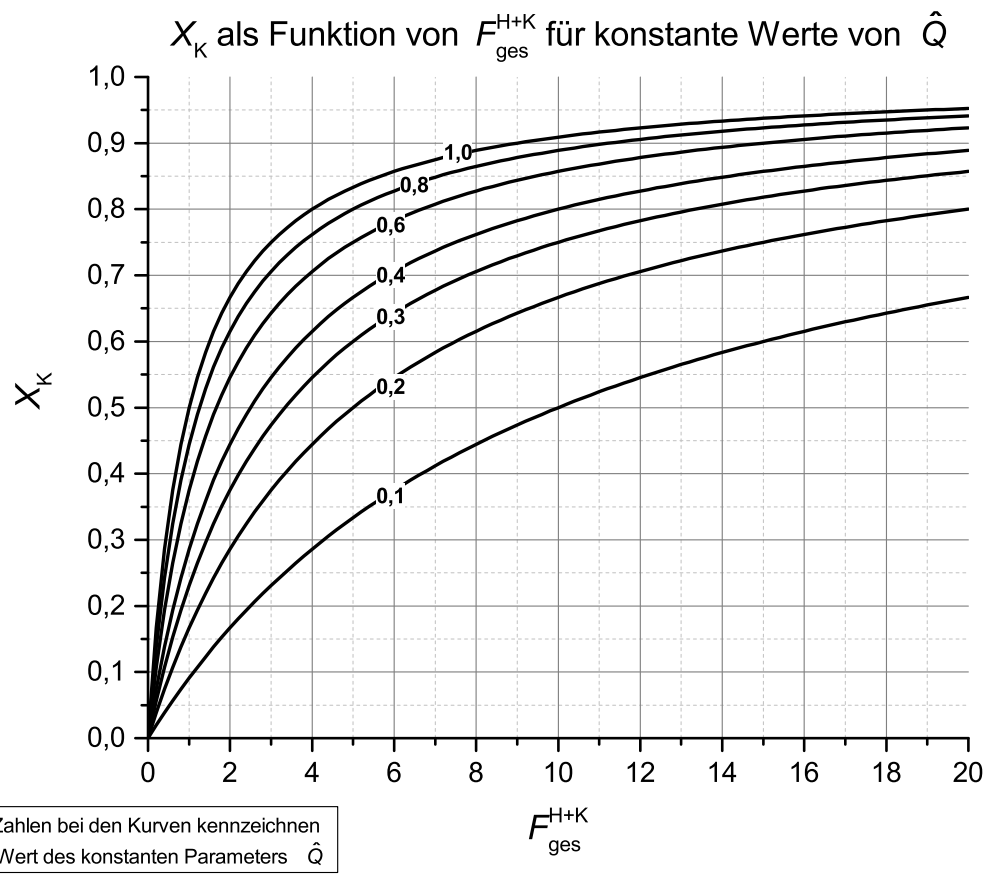

Abbildung 3.26: $X_{\mathrm{K}}$ als Funktion von $F_{\text {ges }}^{\mathrm{H}+\mathrm{K}}$ für konstante Werte von $\widehat{Q}$ 


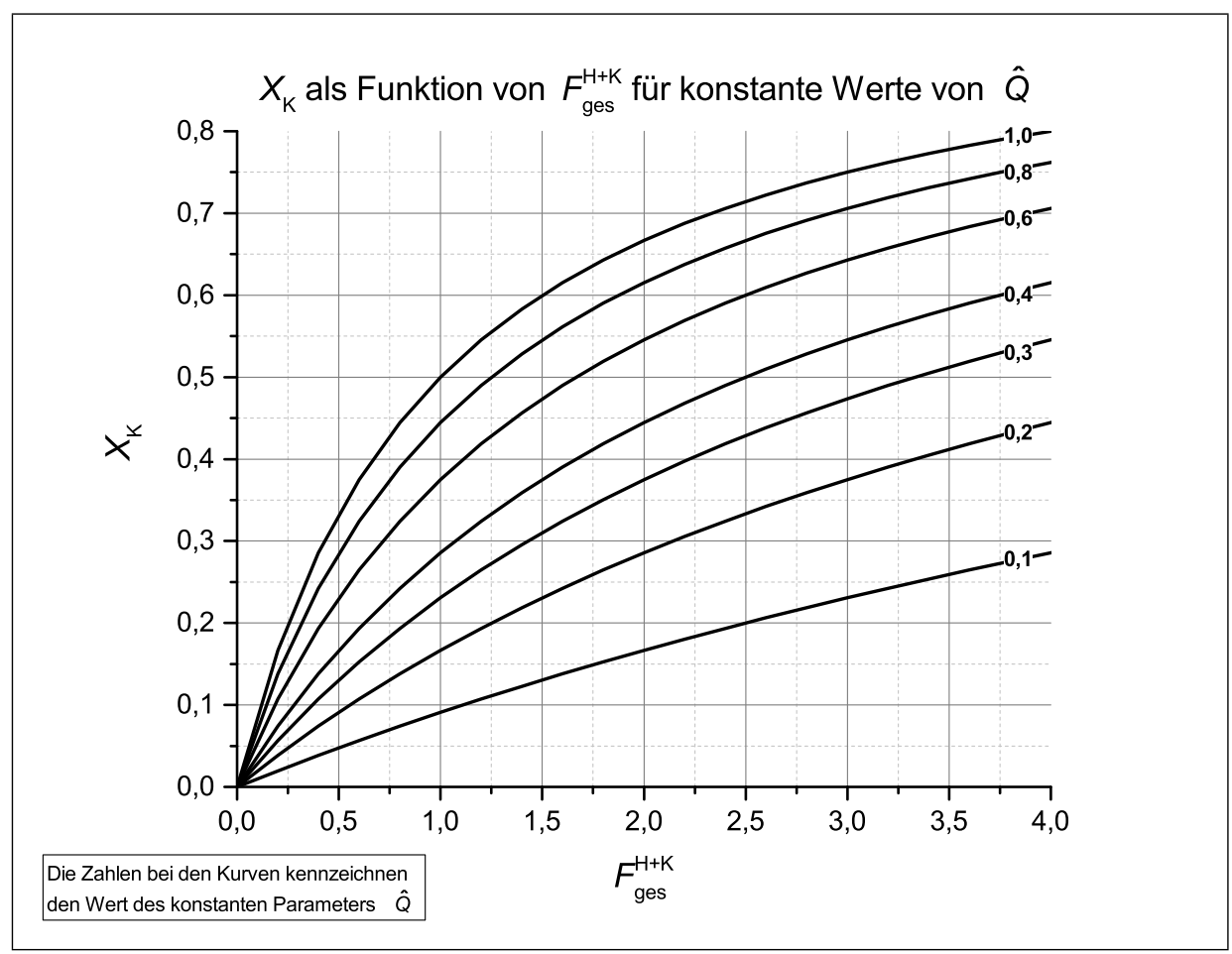

Abbildung 3.27: $X_{\mathrm{K}}$ als Funktion von $F_{\text {ges }}^{\mathrm{H}+\mathrm{K}}$ im Intervall $0 \leq F_{\text {ges }}^{\mathrm{H}+\mathrm{K}} \leq 4$ für konstante Werte von $\widehat{Q}$

\section{Hinweise zu den Diagrammen zur Ermittlung von $\boldsymbol{X}_{\mathrm{H}}$ und $\boldsymbol{X}_{\mathrm{H}}$}

- Auf der Abszisse in den Abbildungen 3.24 bis 3.27 ist $F_{\text {ges }}^{\mathrm{H}+\mathrm{K}}$ aufgetragen. Als konstanter Parameter wurde $\widehat{Q}$ gewählt, da $\widehat{Q}$ ein für die Darstellung günstiges Intervall aufweist.

- Der Ableseweg wird im Folgenden am Beispiel von $F_{\text {ges }}^{\mathrm{H}+\mathrm{K}}$ und $\widehat{Q}=$ konstant beschrieben: Es wird eine Vertikale durch $F_{\text {ges }}^{\mathrm{H}+\mathrm{K}}$ gelegt und mit der korrespondierenden Kurve für $\widehat{Q}=$ konstant geschnitten. Für den ermittelten Schnittpunkt kann $X_{\mathrm{H}}$ bzw. $X_{\mathrm{K}}$ abgelesen werden.

- Aus $F_{\text {ges }}^{\mathrm{H}+\mathrm{K}}=2$ und $\widehat{Q}=0,6$ folgt beispielsweise $X_{\mathrm{H}}=0,45$ und $X_{\mathrm{K}}=0,55$. 
Erstellung von Diagrammen für $\widehat{\boldsymbol{E}}^{\mathbf{H}+\mathrm{K}}$

Zur Visualisierung von $\widehat{E}^{\mathrm{H}+\mathrm{K}}$ werden die Zusammenhänge nach Gleichung (3.92):

$$
\begin{aligned}
& \widehat{E}^{\mathrm{H}+\mathrm{K}}=\widehat{E}^{\mathrm{H}}+X_{\mathrm{K}}\left(1-\widehat{E}^{\mathrm{H}}\right) \\
& \widehat{E}^{\mathrm{H}+\mathrm{K}}=1+X_{\mathrm{H}}\left(\widehat{E}^{\mathrm{H}}-1\right)
\end{aligned}
$$

im Intervall

$$
\begin{aligned}
& 0 \leq \widehat{E}^{\mathrm{H}} \leq 1 \\
& 0 \leq X_{\mathrm{H}} \leq 1 \\
& 0 \leq X_{\mathrm{K}} \leq 1
\end{aligned}
$$

dargestellt.

Abbildung 3.28 und 3.29 zeigen $\widehat{E}^{\mathrm{H}+\mathrm{K}}$ als Funktion von $\widehat{E}^{\mathrm{H}}$ für konstante Werte von $X_{\mathrm{H}}$ bzw. $X_{\mathrm{K}}$.

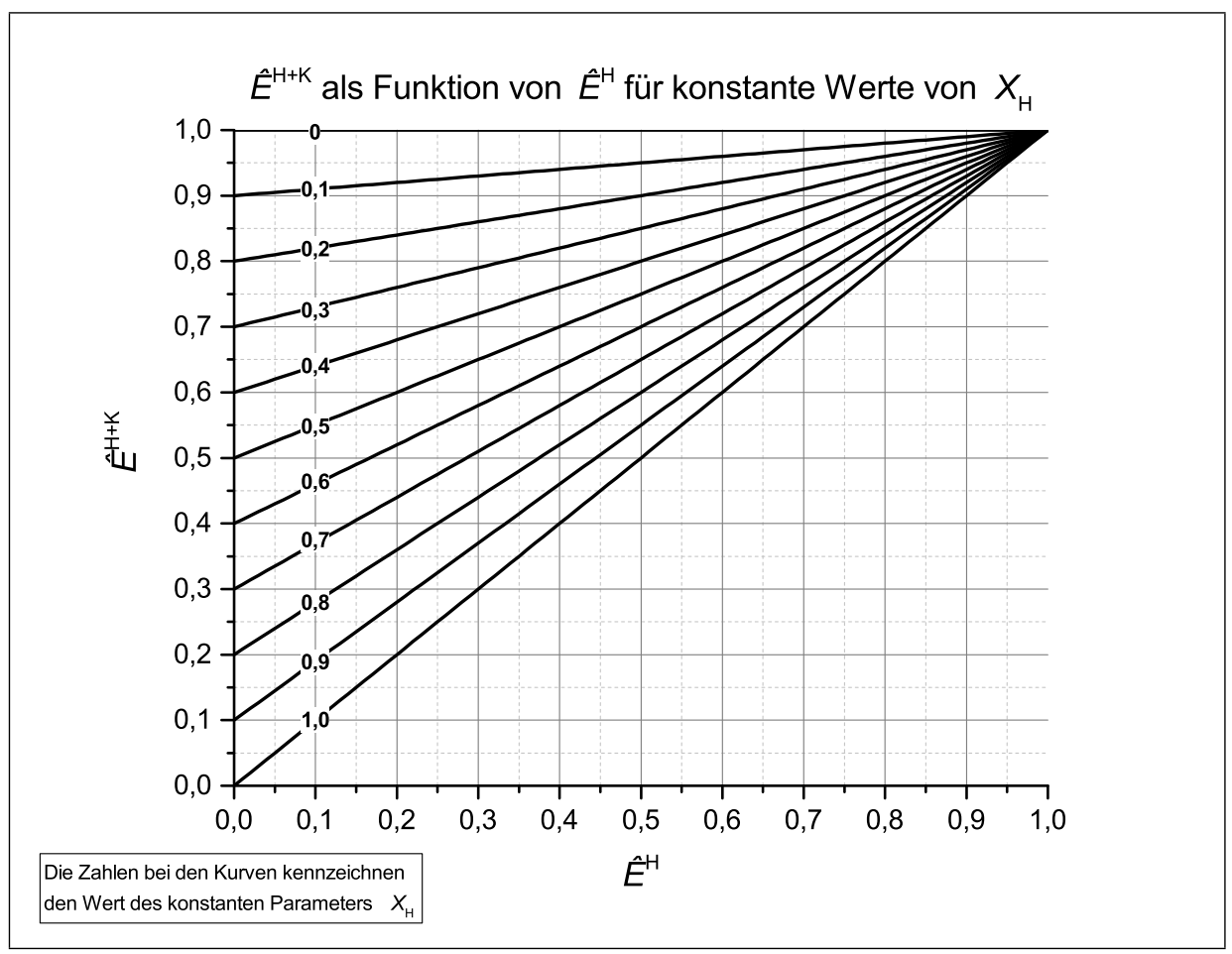

Abbildung 3.28: $\widehat{E}^{\mathrm{H}+\mathrm{K}}$ als Funktion von $\widehat{E}^{\mathrm{H}}$ für konstante Werte von $X_{\mathrm{H}}$ 


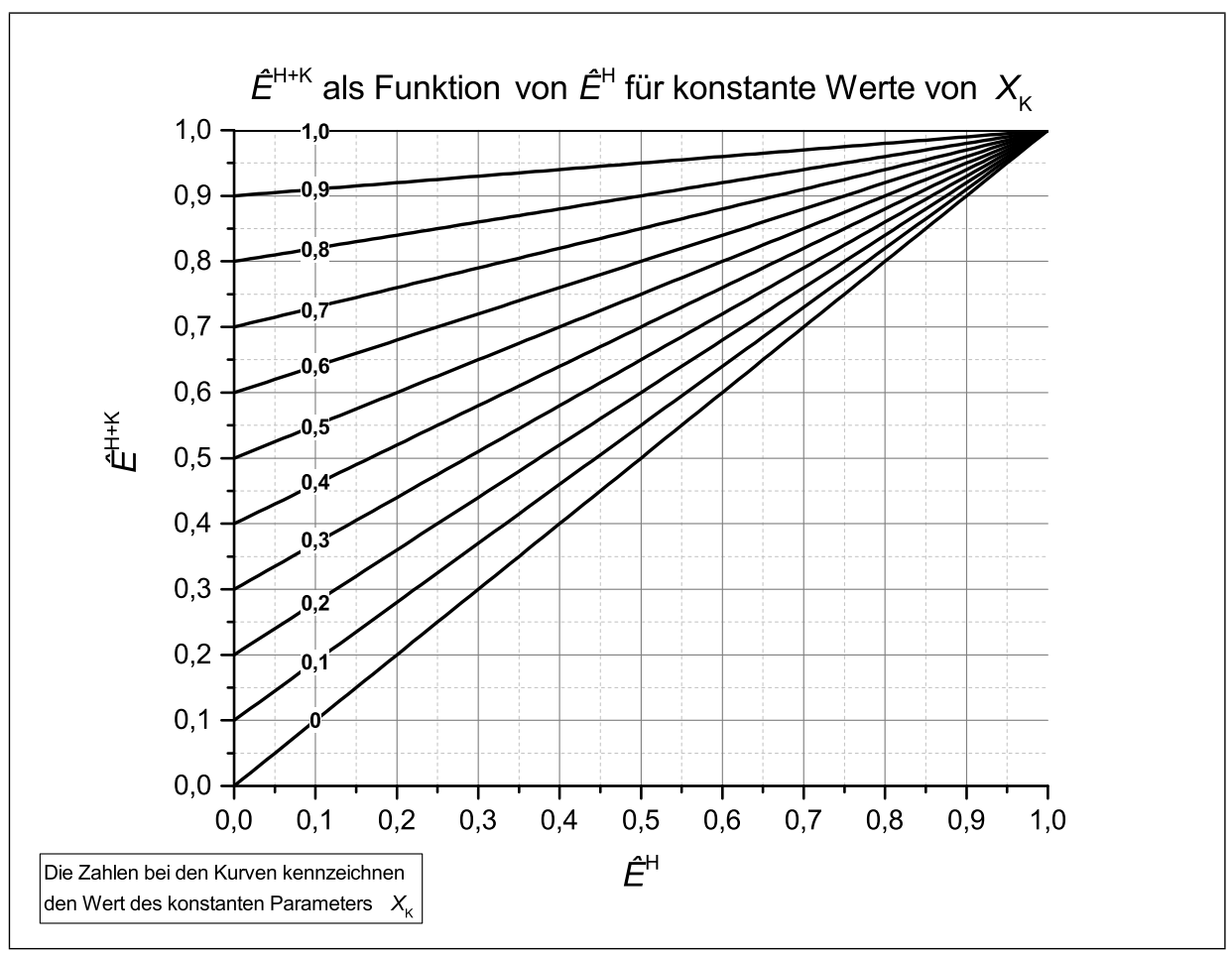

Abbildung 3.29: $\widehat{E}^{\mathrm{H}+\mathrm{K}}$ als Funktion von $\widehat{E}^{\mathrm{H}}$ für konstante Werte von $X_{\mathrm{K}}$

\section{Hinweise zu den Diagrammen zur Ermittlung von $\widehat{\boldsymbol{E}}^{\mathrm{H}+\mathrm{K}}$}

- Die Kurven in Abbildung 3.28 und 3.29 für die Grenze des Intervalls des konstanten Parameters $X_{\mathrm{H}}$ bzw. $X_{\mathrm{K}}$ bei:

$$
X_{\mathrm{H}}=0 \longrightarrow X_{\mathrm{K}}=1
$$

dienen primär als Ablesehilfe. In der Anwendung sind sie von untergeordneter Bedeutung, da es sich hierbei um die theoretischen Grenzfälle $K_{\mathrm{kon}}^{\mathrm{H}} \rightarrow 0$ bzw. $Q_{\mathrm{N}}^{\mathrm{H}} \rightarrow 0$ handelt.

- Die Kurven in Abbildung 3.28 und 3.29 für die Grenze des Intervalls des konstanten Parameters $X_{\mathrm{K}}$ bzw. $X_{\mathrm{H}}$ bei:

$$
X_{\mathrm{K}}=0 \longrightarrow X_{\mathrm{H}}=1
$$

repräsentieren den Fall, dass $K_{\mathrm{kon}}^{\mathrm{K}}=0$ zutrifft. Hierbei handelt es sich nicht um ein theoretisches Szenario. Verwendet ein Unternehmen beispielsweise zur Verfügung stehendes Flusswasser für Kühlzwecke und sind die daraus resultierenden spezifischen Kühlkosten zu vernachlässigen, so gilt $\widehat{E}^{\mathrm{H}+\mathrm{K}}=\widehat{E}^{\mathrm{H}}$, was dem Resultat des vorgestellten Formelwerks im Fall $X_{\mathrm{K}}=0$ entspricht. 
- Der Ableseweg wird im Folgenden am Beispiel von $\widehat{E}^{\mathrm{H}}$ und $X_{\mathrm{H}}=$ konstant beschrieben, gilt jedoch analog für den Fall, dass $X_{\mathrm{K}}$ vorgegeben wäre:

Es wird eine Vertikale durch $\widehat{E}^{\mathrm{H}}$ gelegt und mit der korrespondierenden Kurve für $X_{\mathrm{H}}=$ konstant geschnitten. Für den ermittelten Schnittpunkt kann $\widehat{E}^{\mathrm{H}+\mathrm{K}}$ abgelesen werden.

- Bei einer relativen Energiekosteneinsparung im Heizbetrieb $\widehat{E}^{\mathrm{H}}$ von 0,4 und $X_{\mathrm{H}}=0,5$ folgt beispielsweise $\widehat{E}^{\mathrm{H}+\mathrm{K}}=0,7$.

\subsection{Absolute wirtschaftliche Kennzahlen}

Die formale Zielsetzung der vorliegenden Arbeit ist es, ein Konzept zu entwickeln, das die Abschätzung der relativen Energiekosteneinsparung einer Wärmepumpe im Heiz- und Kühlbetrieb erlaubt. In der Anwendung wird sich jedoch ebenfalls die Frage stellen, mit welchen absoluten Einsparungen, Investitionskosten und Amortisationszeiträumen zu rechnen ist. Die sorgfältige Beantwortung dieser Fragestellungen kann und soll in der vorliegenden Arbeit nicht abgedeckt werden. Um dennoch Aussagen zu den absoluten wirtschaftlichen Größen der in Kapitel 5 vorgestellten Szenarien des Fallbeispiels tätigen zu können, werden im Folgenden stark vereinfachte Zusammenhänge zur Abschätzung der absoluten Einsparung, der Investitionskosten und der Amortisationszeiträumen vorgestellt, jedoch nicht anhand von Diagrammen zugänglich gemacht. Da die abzuleitenden Bestimmungsgleichungen den Charakter einer ersten Näherung besitzen, ist auf die Verwendung von integralen Zusammenhängen verzichtet worden.

\subsubsection{Absolute Energiekosteneinsparung}

Die absolute Energiekosteneinsparung ist, im Gegensatz zu den bisher in Kapitel 3.4 hergeleiteten Größen, keine relative Kennzahl sonder eine absolute Größe, deren Betrag davon abhängt, über welchen Betrachtungszeitraum eine gewisse relative Einsparung realisiert werden kann.

Für einen Betriebspunkt $P_{\mathrm{i}}$ gelte, dass alle den Betriebspunkt beschreibenden technischen und wirtschaftlichen Kennzahlen bzw. Parameter über das zeitliche, dem Betriebspunkt zugeordnete, Intervall $\Delta t_{\mathrm{i}}$ konstant sind. Dann lässt sich beispielsweise die Nutzwärme im Heizbetrieb $Q_{\mathrm{N}}^{\mathrm{H}}\left(P_{\mathrm{i}}\right)$, die im Intervall $\Delta t_{\mathrm{i}}$ vom Wärmepumpenkreislauf abgegeben wird, nach Gleichung (3.50) wie folgt anschreiben:

$$
Q_{\mathrm{N}}^{\mathrm{H}}\left(P_{\mathrm{i}}\right)=\dot{Q}_{\mathrm{N}}^{\mathrm{H}}\left(P_{\mathrm{i}}\right) \Delta t_{\mathrm{i}}
$$


Für die absolute Einsparung $E_{\mathrm{i}}\left(\Delta t_{\mathrm{i}}\right)$, welche über die Dauer von $\Delta t_{\mathrm{i}}$ realisiert wird, folgt daraus je nach Betriebsfall:

- Heizbetrieb:

$$
E_{\mathrm{i}}^{\mathrm{H}}\left(\Delta t_{\mathrm{i}}\right)=G K_{\mathrm{kon}}^{\mathrm{H}}\left(P_{\mathrm{i}}\right) \widehat{E}^{\mathrm{H}}\left(P_{\mathrm{i}}\right)
$$

- Heiz- und Kühlbetrieb:

$$
E_{\mathrm{i}}^{\mathrm{H}+\mathrm{K}}\left(\Delta t_{\mathrm{i}}\right)=G K_{\mathrm{kon}}^{\mathrm{H}+\mathrm{K}}\left(P_{\mathrm{i}}\right) \widehat{E}^{\mathrm{H}+\mathrm{K}}\left(P_{\mathrm{i}}\right)
$$

Liegen im zu betrachtenden Zeitraum $\Delta t_{\mathrm{P}}$ mehrere Betriebspunkte, so gelte weiteres:

$$
\begin{aligned}
\Delta t_{\mathrm{i}} & \in \Delta t_{\mathrm{P}} \\
\sum_{\mathrm{i}} \Delta t_{\mathrm{i}} & =\Delta t_{\mathrm{P}}
\end{aligned}
$$

Dann setzt sich die absolute Energiekosteneinsparung $E\left(\Delta t_{\mathrm{P}}\right)$, welche im Zeitraum $\Delta t_{\mathrm{P}}$ realisiert wird, additiv aus den einzelnen Energiekosteneinsparungen zusammen:

$$
E\left(\Delta t_{\mathrm{P}}\right)=\sum_{\mathrm{i}} E_{\mathrm{i}}\left(\Delta t_{\mathrm{i}}\right)
$$

Wird die absolute Energiekosteneinsparung pro betrachtetem Zeitraum benötigt, beispielsweise zur Ermittlung der jährlichen Energiekosteneinsparung, so ist die absolute Einsparung im Betrachtungszeitraum $\Delta t_{\mathrm{P}}$ mit dem Betrachtungszeitraum zu normieren:

$$
\bar{E}\left(\Delta t_{\mathrm{P}}\right)=\frac{E\left(\Delta t_{\mathrm{P}}\right)}{\Delta t_{\mathrm{P}}}=\frac{\sum_{\mathrm{i}} E_{\mathrm{i}}\left(\Delta t_{\mathrm{i}}\right)}{\sum_{\mathrm{i}} \Delta t_{\mathrm{i}}}
$$

Hinweis: Die Umlegung der absoluten Energiekosteneinsparung pro betrachtetem Zeitraum auf ein Vielfaches des betrachteten Zeitraums, ist nur dann zu empfehlen, wenn der betrachtete Zeitraum tatsächlich repräsentativ ist. Zur Ermittlung der absoluten Einsparung über einen Zeitraum von 3 Jahren darf daher beispielsweise nicht einfach die absolute Einsparung, die in 3 zusammenhängenden Monaten realisiert werden kann, mit dem Faktor 12 multipliziert werden. Vorher ist vielmehr zu prüfen, ob der betrachtete Zeitraum auch tatsächlich mit hinreichender Genauigkeit zur Extrapolation geeignet ist. 


\subsubsection{Investitionskosten}

Zur Berechnung der Investitionskosten eines Wärmepumpenkreislauf $I K_{\mathrm{WP}}$ wird unterstellt, dass sich diese aus einem Anteil $I K_{1}^{\mathrm{H}}$, der proportional zur Heizleistung ist, und einem Anteil $I K_{2}$, der nicht von der Heizleistung abhängt, zusammensetzen. Daraus folgt:

$$
I K_{\mathrm{WP}}=I K_{1}^{\mathrm{H}}\left(\dot{Q}_{\mathrm{N}}^{\mathrm{H}}\right)+I K_{2}
$$

Für die Abschätzung der Investitionskosten wird angenommen:

$$
I K_{1}^{\mathrm{H}}\left(\dot{Q}_{\mathrm{N}}^{\mathrm{H}}\right) \gg I K_{2} \longrightarrow I K_{\mathrm{WP}} \sim I K_{1}^{\mathrm{H}}\left(\dot{Q}_{\mathrm{N}}^{\mathrm{H}}\right)
$$

Der tiefgestellte Index bei $I K_{1}^{\mathrm{H}}$ kann daher entfallen, was auf den im vorliegenden Dokument verwendeten Ansatz zur Berechnung der Investitionskosten eines Wärmepumpenkreislauf führt:

$$
I K_{\mathrm{WP}}:=I K^{\mathrm{H}}\left(\dot{Q}_{\mathrm{N}}^{\mathrm{H}}\right)
$$

Hersteller von Industriewärmepumpen haben im Jahr 2017 die spezifischen von der Heizleistung abhängigen Kosten eines Wärmepumpenkreislauf mit $€ 250$ bis $€ 400$ je kW Heizleistung beziffert. Werden die spezifischen von der Heizleistung abhängigen Investitionskosten eines Wärmepumpenkreislauf mit $K_{\mathrm{WP}}^{\mathrm{IK}}$ bezeichnet, so lassen sich diese daher wie folgt angeben:

$$
\begin{gathered}
K_{\mathrm{WP}}^{\mathrm{IKmin}}=\frac{€ 250}{\mathrm{~kW}} \\
K_{\mathrm{WP}}^{\mathrm{IKmax}}=\frac{€ 400}{\mathrm{~kW}} \\
K_{\mathrm{WP}}^{\mathrm{IKmin}} \leq K_{\mathrm{WP}}^{\mathrm{IK}} \leq K_{\mathrm{WP}}^{\mathrm{IKmax}}
\end{gathered}
$$

Daher ergibt sich für die Berechnung von $I K^{\mathrm{H}}\left(\dot{Q}_{\mathrm{N}}^{\mathrm{H}}\right)$ :

$$
I K^{\mathrm{H}}\left(\dot{Q}_{\mathrm{N}}^{\mathrm{H}}\right)=\dot{Q}_{\mathrm{N}}^{\mathrm{H}} K_{\mathrm{WP}}^{\mathrm{IK}} \quad\left[\dot{Q}_{\mathrm{N}}^{\mathrm{H}}\right]=\mathrm{kW}
$$

was in Gleichung (3.126) eingesetzt:

$$
I K_{\mathrm{WP}}=\dot{Q}_{\mathrm{N}}^{\mathrm{H}} K_{\mathrm{WP}}^{\mathrm{IK}} \quad\left[\dot{Q}_{\mathrm{N}}^{\mathrm{H}}\right]=\mathrm{kW}
$$

den gesuchten Zusammenhang für die Berechnung der Investitionskosten ergibt. 


\subsubsection{Amortisationsdauer}

Die Amortisationsdauer $\Delta t_{\mathrm{A}}$, ausgedrückt in einer Anzahl von Zeiträumen $\Delta t_{\mathrm{P}}$, errechnet sich dadurch, dass die Investitionskosten $I K_{\mathrm{WP}}$ mit der absoluten Einsparung in Zeitraum $\Delta t_{\mathrm{P}}$ normiert wird:

$$
\Delta t_{\mathrm{A}}=\frac{I K_{\mathrm{WP}}}{\bar{E}\left(\Delta t_{\mathrm{P}}\right)}
$$

Werden die Resultate aus Gleichung (3.123) und Gleichung (3.129) in Gleichung (3.130) eingesetzt, so folgt daraus:

$$
\begin{aligned}
\Delta t_{\mathrm{A}} & =\frac{I K_{\mathrm{WP}}}{\bar{E}\left(\Delta t_{\mathrm{P}}\right)}=\frac{\dot{Q}_{\mathrm{N}}^{\mathrm{H}} K_{\mathrm{WP}}^{\mathrm{IK}}}{\frac{E\left(\Delta t_{\mathrm{P}}\right)}{\Delta t_{\mathrm{P}}}} \\
\longrightarrow \Delta t_{\mathrm{A}} & =\Delta t_{\mathrm{P}} \frac{\dot{Q}_{\mathrm{N}}^{\mathrm{H}} K_{\mathrm{WP}}^{\mathrm{IK}}}{E\left(\Delta t_{\mathrm{P}}\right)} \quad\left[\dot{Q}_{\mathrm{N}}^{\mathrm{H}}\right]=\mathrm{kW}
\end{aligned}
$$

Das Resultat aus Gleichung (3.131) lässt sich wie folgt deuten:

- Gilt beispielsweise für den Betrachtungszeitraum $\Delta t_{\mathrm{P}}$ :

$$
\Delta t_{\mathrm{P}}=1 \mathrm{Jahr}
$$

dann gibt $E\left(\Delta t_{\mathrm{P}}\right)$ die jährliche Energiekosteneinsparung an.

- Die Amortisationsdauer $\Delta t_{\mathrm{A}}$, als eine Anzahl von Zeiträumen $\Delta t_{\mathrm{P}}$, wird daher zu einer Anzahl an Jahren, und lässt sich durch Normierung der Investitionskosten mit der jährlichen Einsparung berechnen.

Diese Art der Einführung der Amortisationsdauer erscheint auf den ersten Blick etwas ungewöhnlich. Sie hat jedoch den Vorteil, dass mit beliebigen Zeiträumen $\Delta t_{\mathrm{P}}$ gerechnet werden kann und nicht stillschweigend vorausgesetzt werden muss, dass $\Delta t_{\mathrm{P}}$ ein Jahr beträgt. Die eventuell benötigte Umrechnung auf das gewünschte Zeitformat wird daher am Ende der Berechnung für die Amortisationsdauer $\Delta t_{\mathrm{A}}$ und nicht für die Einsparung $E\left(\Delta t_{\mathrm{P}}\right)$ durchgeführt. 


\section{Zusammenfassung}

In diesem Kapitel werden die für die Bewertung der Leistungsfähigkeit relevanten Kennzahlen aus Kapitel 3 zusammengefasst. Tabellen und Diagramme in höherer Auflösung befinden sich im Anhang.

Für die Zusammenfassung gilt, dass eine einmal vorgestellte Kennzahl für die nachfolgenden Kennzahlen als bekannt anzusehen ist. So wird beispielsweise $\widehat{E}^{\mathrm{H}}$ vor $\widehat{E}^{\mathrm{H}+\mathrm{K}}$ aufgeführt. Daher wird der Weg zur Ermittlung von $\widehat{E}^{\mathrm{H}}$ bei der Zusammenfassung von $\widehat{E}^{\mathrm{H}+\mathrm{K}}$ nicht mehr separat beschrieben.

\subsection{Technische Kennzahlen}

\section{Maximaler COP im Heizbetrieb $\mathrm{COP}_{\max }^{\mathrm{H}}$}

\section{Beschreibung}

Der COP der sich einstellt, wenn in Gleichung (2.7) die Temperaturen der Quelle und der Senke nach Gleichung (3.1) eingesetzt werden. Nach Gleichung (3.2) gegeben durch:

$$
C O P_{\max }^{\mathrm{H}}=\frac{T_{\mathrm{H}}}{T_{\mathrm{H}}-T_{\mathrm{N}}}=\frac{T_{\mathrm{S}}+\Delta T_{\mathrm{WT}}}{T_{\mathrm{S}}+2 \Delta T_{\mathrm{WT}}-T_{\mathrm{Q}}}
$$

\section{Ableitung auf Basis der ermittelten Zusammenhänge unter Verwendung des Temperaturfaktors $\boldsymbol{X}_{\mathrm{T}}$}

1. Ermittlung des maximalen COPs im Heizbetrieb bei $\Delta T_{\mathrm{WT}}=0{ }^{\circ} \mathrm{C}$

- Berechnung:

$$
C O P_{0}^{\mathrm{H}}=\frac{T_{\mathrm{S}}}{T_{\mathrm{S}}-T_{\mathrm{Q}}}
$$

- Abbildung 3.6 und 3.7 oder 3.8.

- Abbildung A.3 und A.4 oder A.5. 
2. Ermittlung der dimensionslosen Temperaturdifferenz $\widehat{\Delta T}$

- Berechnung:

(a) Temperaturdifferenz zwischen Senke und Quelle bestimmen:

$$
\Delta T_{\mathrm{SQ}}=T_{\mathrm{S}}-T_{\mathrm{Q}}
$$

(b) Charakteristische Temperaturdifferenz in den Wärmetauschern $\Delta T_{\mathrm{WT}}$ festlegen.

(c) Dimensionslose Temperaturdifferenz berechnen:

$$
\widehat{\Delta T}=\frac{\Delta T_{\mathrm{WT}}}{\Delta T_{\mathrm{SQ}}}
$$

- Abbildung 3.4 und 3.5.

- Abbildung A.1 und A.2.

3. Ermittlung des Temperaturfaktors $X_{\mathrm{T}}$

- Berechnung:

$$
X_{\mathrm{T}}=1+\frac{\widehat{\Delta T}}{C O P_{0}^{\mathrm{H}}}\left(\frac{1-2 C O P_{0}^{\mathrm{H}}}{1+2 \widehat{\Delta T}}\right)
$$

- Abbildung 3.9 .

- Abbildung A.6.

4. Ermittlung des maximalen COPs im Heizbetrieb $C O P_{\max }^{\mathrm{H}}$

- Berechnung:

$$
C O P_{\max }^{\mathrm{H}}=X_{\mathrm{T}} C O P_{0}^{\mathrm{H}}
$$

- Abbildung 3.10 und 3.11.

- Abbildung A.7 und A.8.

\section{Ableitung auf Basis der ermittelten Zusammenhänge unter Verwendung des gemittelten maximalen COPs im Heizbetrieb $\overline{C O P}_{\max }^{\mathrm{H}}$}

1. Ermittlung des gemittelten maximalen COPs im Heizbetrieb $\overline{C O P_{\max }^{\mathrm{H}}}$

- Abbildung 3.14 und Tabelle 3.1.

- Abbildung A.9 und Tabelle A.1. 


\section{Realer COP im Heizbetrieb $C O P_{\text {real }}^{\mathrm{H}}$}

\section{Beschreibung}

Der Quotient aus dem, im realen Prozess, bei hohem Temperaturniveau abgegeben Wärmestrom und dem zugeführten Arbeitsstrom. Nach Gleichung (3.34) gegeben durch:

$$
C O P_{\text {real }}^{\mathrm{H}}=\frac{\dot{Q}_{34}^{\mathrm{r}}}{\dot{W}_{23}^{\mathrm{r}}}=\frac{\dot{Q}_{\mathrm{N}}^{\mathrm{H}}}{\dot{W}_{23}^{\mathrm{r}}}
$$

\section{Ableitung auf Basis der ermittelten Zusammenhänge}

1. Ermittlung des realen COPs im Heizbetrieb $C O P_{\text {real }}^{\mathrm{H}}$

- Berechnung:

$$
C O P_{\text {real }}^{\mathrm{H}}=\eta_{\mathrm{WP}} C O P_{\max }^{\mathrm{H}}
$$

- Abbildung 3.15 und 3.15 .

- Abbildung A.10 und A.10.

\section{Quotient der Nutzwärmeströme $\widehat{Q}$}

\section{Beschreibung}

Der Quotient aus dem, im realen Prozess, bei niederem Temperaturniveau aufgenommenen Wärmestrom und dem bei hohem Temperaturniveau abgegeben Wärmestrom. Nach Gleichung (3.42) gegeben durch:

$$
\widehat{Q}=\frac{\dot{Q}_{\mathrm{N}}^{\mathrm{K}}}{\dot{Q}_{\mathrm{N}}^{\mathrm{H}}}
$$

\section{Ableitung auf Basis der ermittelten Zusammenhänge}

1. Ermittlung des Quotienten der Nutzwärmeströme $\widehat{Q}$

- Berechnung:

$$
\widehat{Q}=1-\frac{1}{C O P_{\text {real }}^{\mathrm{H}}}
$$

- Abbildung 3.18.

- Abbildung A.12. 


\subsection{Relative wirtschaftliche Kennzahlen}

\section{Wirtschaftlichkeitsgrenze im Heizbetrieb $\left.F_{\text {ges }}^{\mathbf{H}}\right|_{\max }$}

\section{Beschreibung}

Jene Parameterkonstellation bei deren Unterschreitung der wirtschaftliche Einsatz einer Wärmepumpe im Heizbetrieb nicht mehr möglich ist. Nach Gleichung (3.69) und (3.70) gegeben durch:

$$
\begin{array}{r}
F_{\text {ges }}^{\mathrm{H}}<C O P_{\max }^{\mathrm{H}} \\
\left.F_{\text {ges }}^{\mathrm{H}}\right|_{\max }=C O P_{\max }^{\mathrm{H}}
\end{array}
$$

\section{Ableitung auf Basis der ermittelten Zusammenhänge}

1. Ermittlung des Gesamteinflussfaktors im Heizbetrieb $F_{\text {ges }}^{\mathrm{H}}$

- Berechnung:

$$
F_{\mathrm{ges}}^{\mathrm{H}}=\frac{K_{\mathrm{WP}}^{\mathrm{H}}}{K_{\mathrm{kon}}^{\mathrm{H}}} \frac{\eta_{\mathrm{kon}}^{\mathrm{H}}}{\eta_{\mathrm{WP}}}
$$

- Ablesevorgang aus Diagrammen vom Typ $F_{\mathrm{B}}^{\mathrm{A}}$ :

(a) Korrespondierende Größen aus:

$$
F_{\mathrm{B}}^{\mathrm{A}}=\frac{K_{\mathrm{A}}}{K_{\mathrm{B}}} \frac{\eta_{\mathrm{B}}}{\eta_{\mathrm{A}}} \longleftrightarrow F_{\text {ges }}^{\mathrm{H}}=\frac{K_{\mathrm{WP}}^{\mathrm{H}}}{K_{\mathrm{kon}}^{\mathrm{H}}} \frac{\eta_{\mathrm{kon}}^{\mathrm{H}}}{\eta_{\mathrm{WP}}}
$$

und:

$$
\begin{aligned}
\eta_{\mathrm{A}} & =\eta_{\mathrm{WP}} & \eta_{\mathrm{B}} & =\eta_{\mathrm{kon}}^{\mathrm{H}} \\
K_{\mathrm{A}} & =K_{\mathrm{WP}}^{\mathrm{H}} & K_{\mathrm{B}} & =K_{\mathrm{kon}}^{\mathrm{H}}
\end{aligned}
$$

ermitteln.

(b) Quotient aus den spezifischen Kosten $K_{\mathrm{WP}}^{\mathrm{H}}$ und $K_{\mathrm{kon}}^{\mathrm{H}}$ bestimmen.

(c) Quotient aus dem Konversionswirkungsgrad im Heizbetrieb $\eta_{\mathrm{kon}}^{\mathrm{H}}$ und dem Gütegrad $\eta_{\mathrm{WP}}$ aus:

- Abbildung 3.19 bzw. 3.20 ablesen.

- Abbildung A.13 bzw. A.14 ablesen.

(d) Gesamteinflussfaktor im Heizbetrieb $F_{\text {ges }}^{\mathrm{H}}$ aus:

- Abbildung 3.21 bzw. 3.22 ablesen.

- Abbildung A.15 bzw. A.16 ablesen. 


\section{Relative Energiekosteneinsparung im Heizbetrieb $\widehat{\boldsymbol{E}}^{\mathrm{H}}$}

\section{Beschreibung}

Die Differenz aus den Wärmebereitstellungskosten der Vergleichstechnologie und den Wärmebereitstellungskosten mit einer Wärmepumpe, normiert mit den Wärmebereitstellungskosten der Vergleichstechnologie. Nach Gleichung (3.77) gegeben durch:

$$
\widehat{E}^{\mathrm{H}}=\frac{G K_{\mathrm{kon}}^{\mathrm{H}}-G K_{\mathrm{WP}}^{\mathrm{H}}}{G K_{\mathrm{kon}}^{\mathrm{H}}}
$$

\section{Ableitung auf Basis der ermittelten Zusammenhänge}

1. Ermittlung der relativen Energiekosteneinsparung im Heizbetrieb $\widehat{E}^{\mathrm{H}}$

- Berechnung:

$$
\widehat{E}^{\mathrm{H}}=1-\frac{F_{\mathrm{ges}}^{\mathrm{H}}}{C O P_{\max }^{\mathrm{H}}}
$$

- Abbildung 3.23.

- Abbildung A.17.

\section{Relative Energiekosteneinsparung im Heiz- und Kühlbetrieb $\widehat{\boldsymbol{E}}^{\mathrm{H}+\mathrm{K}}$}

\section{Beschreibung}

Die Differenz aus den Gesamtkosten beim Heizen und Kühlen mit der Vergleichstechnologie und den Gesamtkosten beim Heizen und Kühlen mit einer Wärmepumpe, normiert mit den Gesamtkosten beim Heizen und Kühlen mit der Vergleichstechnologie. Nach Gleichung (3.81) gegeben durch:

$$
\widehat{E}^{\mathrm{H}+\mathrm{K}}=\frac{G K_{\mathrm{kon}}^{\mathrm{H}}+G K_{\mathrm{kon}}^{\mathrm{K}}-G K_{\mathrm{WP}}^{\mathrm{H}+\mathrm{K}}}{G K_{\mathrm{kon}}^{\mathrm{H}}+G K_{\mathrm{kon}}^{\mathrm{K}}}
$$




\section{Ableitung auf Basis der ermittelten Zusammenhänge}

1. Ermittlung des Gesamteinflussfaktors im Heiz- und Kühlbetrieb $F_{\text {ges }}^{\mathrm{H}+\mathrm{K}}$

- Berechnung:

$$
F_{\text {ges }}^{\mathrm{H}+\mathrm{K}}=\frac{K_{\mathrm{kon}}^{\mathrm{K}}}{K_{\mathrm{kon}}^{\mathrm{H}}} \frac{\eta_{\mathrm{kon}}^{\mathrm{H}}}{\eta_{\mathrm{kon}}^{\mathrm{K}}}
$$

\section{Fall 1:}

Die Vergleichstechnologie mit der gekühlt wird entspricht einer KaltdampfKompressions-Kälteanlage mit bekanntem $C O P_{\text {real }}^{\mathrm{Kv}}$.

Es ist zu setzen:

$$
\eta_{\mathrm{kon}}^{\mathrm{K}}=1 \quad K_{\mathrm{kon}}^{\mathrm{K}}=\frac{K_{\mathrm{el}}}{C O P_{\mathrm{real}}^{\mathrm{Kv}}}
$$

\section{Fall 2:}

Alles was nicht unter Fall 1 fällt. Die Größen $\eta_{\text {kon }}^{\mathrm{K}}$ und $K_{\text {kon }}^{\mathrm{K}}$ sind passend zur realen betrieblichen Situation zu wählen.

- Ablesevorgang aus Diagrammen vom Typ $F_{\mathrm{B}}^{\mathrm{A}}$ :

(a) Korrespondierende Größen aus:

$$
F_{\mathrm{B}}^{\mathrm{A}}=\frac{K_{\mathrm{A}}}{K_{\mathrm{B}}} \frac{\eta_{\mathrm{B}}}{\eta_{\mathrm{A}}} \longleftrightarrow F_{\text {ges }}^{\mathrm{H}+\mathrm{K}}=\frac{K_{\mathrm{kon}}^{\mathrm{K}}}{K_{\text {kon }}^{\mathrm{H}}} \frac{\eta_{\text {kon }}^{\mathrm{H}}}{\eta_{\text {kon }}^{\mathrm{K}}}
$$

Fall 1:

$$
\begin{array}{ll}
\eta_{\mathrm{A}}=\eta_{\mathrm{kon}}^{\mathrm{K}}=1 & \eta_{\mathrm{B}}=\eta_{\mathrm{kon}}^{\mathrm{H}} \\
K_{\mathrm{A}}=K_{\mathrm{kon}}^{\mathrm{H}} & K_{\mathrm{B}}=K_{\mathrm{kon}}^{\mathrm{K}}=\frac{K_{\mathrm{el}}}{C O P_{\mathrm{real}}^{\mathrm{Kv}}}
\end{array}
$$

Fall 2:

$$
\begin{aligned}
\eta_{\mathrm{A}} & =\eta_{\mathrm{kon}}^{\mathrm{K}} & \eta_{\mathrm{B}} & =\eta_{\mathrm{kon}}^{\mathrm{H}} \\
K_{\mathrm{A}} & =K_{\mathrm{kon}}^{\mathrm{H}} & K_{\mathrm{B}} & =K_{\mathrm{kon}}^{\mathrm{K}}
\end{aligned}
$$

ermitteln.

(b) Quotient aus den spezifischen Kosten $K_{\text {kon }}^{\mathrm{K}}$ und $K_{\text {kon }}^{\mathrm{H}}$ bestimmen.

(c) Quotient aus dem Konversionswirkungsgrad im Heizbetrieb $\eta_{\mathrm{kon}}^{\mathrm{H}}$ und dem Konversionswirkungsgrad im Kühlbetrieb $\eta_{\mathrm{kon}}^{\mathrm{K}}$ aus:

- Abbildung 3.19 bzw. 3.20 ablesen.

- Abbildung A.13 bzw. A.14 ablesen.

(d) Gesamteinflussfaktor im Heiz- und Kühlbetrieb $F_{\text {ges }}^{\mathrm{H}+\mathrm{K}}$ aus:

- Abbildung 3.21 bzw. 3.22 ablesen.

- Abbildung A.15 bzw. A.16 ablesen. 
2. Ermittlung des Heizkostenfaktors $X_{\mathrm{H}}$ oder des Kühlkostenfaktors $X_{\mathrm{K}}$

- Berechnung:

$$
\begin{aligned}
& X_{\mathrm{H}}=\frac{1}{1+\widehat{Q} F_{\mathrm{ges}}^{\mathrm{H}+\mathrm{K}}}=1-X_{\mathrm{K}} \\
& X_{\mathrm{K}}=\frac{1}{1+\frac{1}{\widehat{Q}} \frac{1}{F_{\mathrm{ges}}^{\mathrm{H}+\mathrm{K}}}}=1-X_{\mathrm{H}}
\end{aligned}
$$

- Abbildung 3.24 und 3.25 oder Abbildung 3.26 und 3.27 .

- Abbildung A.18 und A.19 oder Abbildung A.20 und A.21.

3. Ermittlung der relativen Energiekosteneinsparung im Heiz- und Kühlbetrieb $\widehat{E}^{\mathrm{H}+\mathrm{K}}$

- Berechnung:

$$
\begin{aligned}
& \widehat{E}^{\mathrm{H}+\mathrm{K}}=\widehat{E}^{\mathrm{H}}+X_{\mathrm{K}}\left(1-\widehat{E}^{\mathrm{H}}\right) \\
& \widehat{E}^{\mathrm{H}+\mathrm{K}}=1+X_{\mathrm{H}}\left(\widehat{E}^{\mathrm{H}}-1\right)
\end{aligned}
$$

- Abbildung 3.28 oder 3.29 .

- Abbildung A.22 oder A.23. 


\subsection{Absolute wirtschaftliche Kennzahlen}

\section{Absolute Energiekosteneinsparung $E\left(\Delta t_{\mathrm{P}}\right)$}

\section{Beschreibung}

Die absolute Energiekosteneinsparung, welche im Zeitraum $\Delta t_{\mathrm{P}}$ realisiert wird. Nach Gleichung (3.122) gegeben durch:

$$
E\left(\Delta t_{\mathrm{P}}\right)=\sum_{\mathrm{i}} E_{\mathrm{i}}\left(\Delta t_{\mathrm{i}}\right)
$$

Kann nach Gleichung (3.123) auch auf den Betrachtungszeitraum bezogen angegeben werden:

$$
\bar{E}\left(\Delta t_{\mathrm{P}}\right)=\frac{E\left(\Delta t_{\mathrm{P}}\right)}{\Delta t_{\mathrm{P}}}=\frac{\sum_{\mathrm{i}} E_{\mathrm{i}}\left(\Delta t_{\mathrm{i}}\right)}{\sum_{\mathrm{i}} \Delta t_{\mathrm{i}}}
$$

\section{Ableitung auf Basis der ermittelten Zusammenhänge}

1. Ermittlung der absoluten Einsparung $E_{\mathrm{i}}\left(\Delta t_{\mathrm{i}}\right)$ für alle Teilintervalle $\Delta t_{\mathrm{i}} \in \Delta t_{\mathrm{P}}$

- Berechnung:

- Heizbetrieb:

$$
E_{\mathrm{i}}^{\mathrm{H}}\left(\Delta t_{\mathrm{i}}\right)=G K_{\mathrm{kon}}^{\mathrm{H}}\left(P_{\mathrm{i}}\right) \widehat{E}^{\mathrm{H}}\left(P_{\mathrm{i}}\right)
$$

- Heiz- und Kühlbetrieb:

$$
E_{\mathrm{i}}^{\mathrm{H}+\mathrm{K}}\left(\Delta t_{\mathrm{i}}\right)=G K_{\mathrm{kon}}^{\mathrm{H}+\mathrm{K}}\left(P_{\mathrm{i}}\right) \widehat{E}^{\mathrm{H}+\mathrm{K}}\left(P_{\mathrm{i}}\right)
$$

2. Ermittlung der absoluten Einsparung im Betrachtungszeitraum $E\left(\Delta t_{\mathrm{P}}\right)$

- Berechnung:

$$
E\left(\Delta t_{\mathrm{P}}\right)=\sum_{\mathrm{i}} E_{\mathrm{i}}\left(\Delta t_{\mathrm{i}}\right)
$$

3. Ermittlung der mittleren Einsparung im Betrachtungszeitraum $\bar{E}\left(\Delta t_{\mathrm{P}}\right)$

- Berechnung:

$$
\bar{E}\left(\Delta t_{\mathrm{P}}\right)=\frac{E\left(\Delta t_{\mathrm{P}}\right)}{\Delta t_{\mathrm{P}}}=\frac{\sum_{\mathrm{i}} E_{\mathrm{i}}\left(\Delta t_{\mathrm{i}}\right)}{\sum_{\mathrm{i}} \Delta t_{\mathrm{i}}}
$$




\section{Investitionskosten $I K_{\mathrm{WP}}$}

\section{Beschreibung}

Die Kosten, die mit der Anschaffung einer Wärmepumpe und deren Integration in die betriebliche Umgebung einhergehen. Nach Gleichung (3.126) abgeschätzt mit:

$$
I K_{\mathrm{WP}}=I K^{\mathrm{H}}\left(\dot{Q}_{\mathrm{N}}^{\mathrm{H}}\right)
$$

\section{Ableitung auf Basis der ermittelten Zusammenhänge}

1. Ermittlung der spezifischen Investitionskosten $K_{\mathrm{WP}}^{\mathrm{IK}}$

- Berechnung:

$$
\begin{gathered}
K_{\mathrm{WP}}^{\mathrm{IKmin}}=\frac{€ 250}{\mathrm{~kW}} \\
K_{\mathrm{WP}}^{\mathrm{IKmax}}=\frac{€ 400}{\mathrm{~kW}} \\
K_{\mathrm{WP}}^{\mathrm{IKmin}} \leq K_{\mathrm{WP}}^{\mathrm{IK}} \leq K_{\mathrm{WP}}^{\mathrm{IK} m a x}
\end{gathered}
$$

2. Ermittlung der Investitionskosten $I K_{\mathrm{WP}}$

- Berechnung:

$$
I K_{\mathrm{WP}}=\dot{Q}_{\mathrm{N}}^{\mathrm{H}} K_{\mathrm{WP}}^{\mathrm{IK}} \quad\left[\dot{Q}_{\mathrm{N}}^{\mathrm{H}}\right]=\mathrm{kW}
$$

\section{Amortisationsdauer $\Delta t_{\mathrm{A}}$}

\section{Beschreibung}

Jener Zeitbereich, den eine Wärmepumpe im Betrieb sein muss, damit das Verhältnis der Investitionskosten zu den realisierten absoluten Energiekosteneinsparungen 1 beträgt. Nach Gleichung (3.130) gegeben durch:

$$
\Delta t_{\mathrm{A}}=\frac{I K_{\mathrm{WP}}}{\bar{E}\left(\Delta t_{\mathrm{P}}\right)}
$$

\section{Ableitung auf Basis der ermittelten Zusammenhänge}

1. Ermittlung der Amortisationsdauer $\Delta t_{\mathrm{A}}$

- Berechnung:

$$
\Delta t_{\mathrm{A}}=\Delta t_{\mathrm{P}} \frac{\dot{Q}_{\mathrm{N}}^{\mathrm{H}} K_{\mathrm{WP}}^{\mathrm{IK}}}{E\left(\Delta t_{\mathrm{P}}\right)} \quad\left[\dot{Q}_{\mathrm{N}}^{\mathrm{H}}\right]=\mathrm{kW}
$$




\section{Anwendung}

In diesem Kapitel wird die Anwendung des Bewertungskonzepts auf verschiedene Problemstellungen anhand eines Fallbeispiels dargestellt. Es wird sowohl der direkte Fall, d.h. die Berechnung der relativen Energiekosteneinsparung aus den dafür benötigen Parametern, als auch der inverse Fall betrachtet. Beim inversen Fall ist es das Ziel, mögliche Parameterkonstellationen zur Erreichung einer bestimmten Kennzahlausprägung festzustellen oder die Sensitivität einer Kennzahl zu ermitteln. Beide Möglichkeiten werden in diesem Kapitel behandelt. Die absoluten wirtschaftlichen Kennzahlen kommen im Rahmen des Fallbeispiels ebenfalls zum Einsatz. Es gelten jedoch die Limitierungen aus Kapitel 3.5.

\subsection{Ausgangssituation}

Ein Unternehmen möchte überprüfen, ob eine Wärmepumpe wirtschaftlich sinnvoll in die betrieblichen Rahmenbedingungen integriert werden kann. Als Quelle für eine mögliche Wärmepumpe ist die Kühlung eines Niederdruckkondensators, wie in Abbildung 5.1 dargestellt, ausgewählt worden. Im Ist-Zustand wird der vom Kondensator kommende Massenstrom über einen Wärmetauscher geführt, dabei von der Temperatur $\vartheta_{1}$ auf die Temperatur $\vartheta_{2}$ abgekühlt und anschließend zurück zum Kondensator geleitet. Für die eigentliche Kühlung wird ein luftgekühlter Sekundärkreislauf eingesetzt, dessen Austausch, aufgrund von mangelnder Kühlleistung im Sommer, im Unternehmen diskutiert wird. Um eine hohe Sicherheit in der Prozessführung, unabhängig von der Außentemperatur, gewährleisten zu können, wird der Einsatz einer Kältemaschine in Betracht gezogen.

Auf der Seite der Senke möchte das Unternehmen Sattdampf bei mindestens $110{ }^{\circ} \mathrm{C}$ erzeugen. Falls sich dies wirtschaftlich nicht darstellen ließe, stünden drei weitere Wärmesenken mit einer Wärmenutzungstemperatur von $70^{\circ} \mathrm{C}, 85^{\circ} \mathrm{C}$ und $95^{\circ} \mathrm{C}$ zur Verfügung.

Im Rahmen einer ersten Abschätzung sollen die Kühlkosten im Status Quo nicht berücksichtigt werden. Bei vielversprechenden Integrationskonzepten ist jedoch der Fall, dass die augenblicklich installierte Luftkühlung durch eine Kälteanlage ersetzt wird, zu berücksichtigen. 


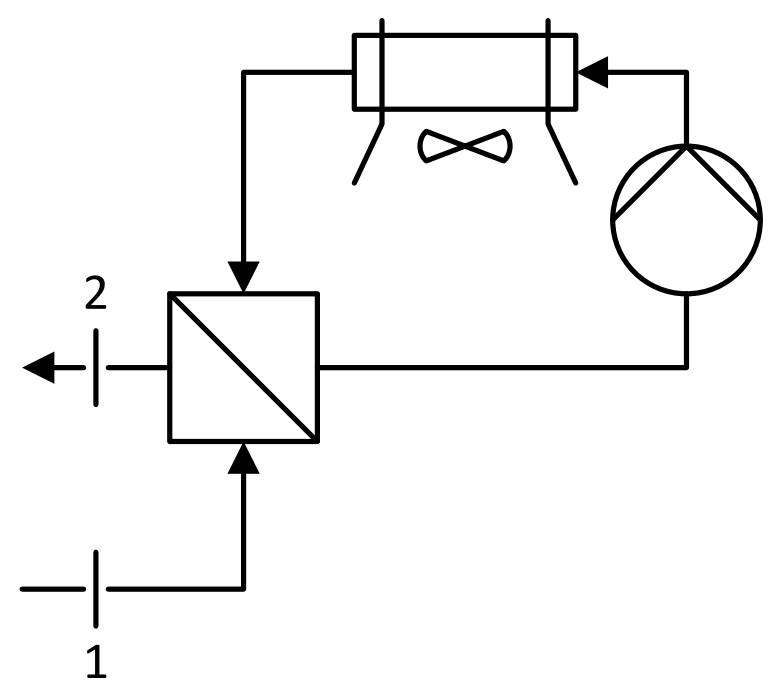

Abbildung 5.1: Fallbeispiel: Skizze der Quelle

Die vom Unternehmen als repräsentativ befundenen Randbedingungen der Integration einer Wärmepumpe zeigt Tabelle 5.1. Die Werte für den Gütegrad sowie die charakteristische Temperaturdifferenz $\Delta T_{\mathrm{WT}}$ sind geschätzt worden.

Tabelle 5.1: Fallbeispiel: Betriebliche Randbedingungen

\begin{tabular}{lll}
\hline \multicolumn{1}{c}{ Quelle } & Vergleichstechnologie & \multicolumn{1}{c}{ Wärmepumpe } \\
\hline$\vartheta_{1}=65^{\circ} \mathrm{C}$ & $K_{\text {kon }}^{\mathrm{H}}=€ 25 / \mathrm{MWh}$ & $K_{\mathrm{el}}=€ 60 / \mathrm{MWh}$ \\
$\vartheta_{2}=35^{\circ} \mathrm{C}$ & $\eta_{\text {kon }}^{\mathrm{H}}=0,85$ & $\eta_{\mathrm{WP}}=0,5$ \\
$\dot{Q}_{12}=850 \mathrm{~kW}$ & & $\Delta T_{\mathrm{WT}}=5{ }^{\circ} \mathrm{C}$ \\
\hline
\end{tabular}

Hinweis: In diesem Kapitel gilt:

- Abgelesene Werte werden mit dem Symbol $\Rightarrow$ gekennzeichnet.

- Errechnete Werte werden mit dem Symbol $\rightarrow$ gekennzeichnet.

- Dezimalstellen werden mit einen Komma gekennzeichnet.

- Der Punkt wird als Tausendertrennzeichen verwendet.

- Einmal vorgestellte Ablese- bzw. Berechnungsvorgänge werden bei erneuter Anwendung in Aufzählungsform dokumentiert. 


\subsection{Direkte Ermittlung von $\widehat{\boldsymbol{E}}^{\mathrm{H}}$}

Bei der direkten Ermittlung der relativen Energiekosteneinsparung im Heizbetrieb $\widehat{E}^{\mathrm{H}}$ sind zuerst die technischen Kennzahlen zu bestimmen.

In Analogie zu den Ausführungen in Kapitel 3.2, wird für die Temperatur der Senke $\vartheta_{\mathrm{S}}$ und die Temperatur der Quelle $\vartheta_{\mathrm{Q}}$ gesetzt:

$$
\vartheta_{\mathrm{S}}=110{ }^{\circ} \mathrm{C} \quad \vartheta_{\mathrm{Q}}=35^{\circ} \mathrm{C}
$$

Ermittlung des maximalen COPs im Heizbetrieb bei $\Delta T_{\mathrm{WT}}=0{ }^{\circ} \mathrm{C}$

Unter Verwendung der Temperaturen aus Gleichung (5.1) lässt sich $C O P_{0}^{\mathrm{H}}$ aus Abbildung 5.2 bestimmen:

$$
\begin{gathered}
\vartheta_{\mathrm{S}}=110{ }^{\circ} \mathrm{C} \quad \vartheta_{\mathrm{Q}}=35{ }^{\circ} \mathrm{C} \\
\Rightarrow C O P_{0}^{\mathrm{H}}=5,2
\end{gathered}
$$

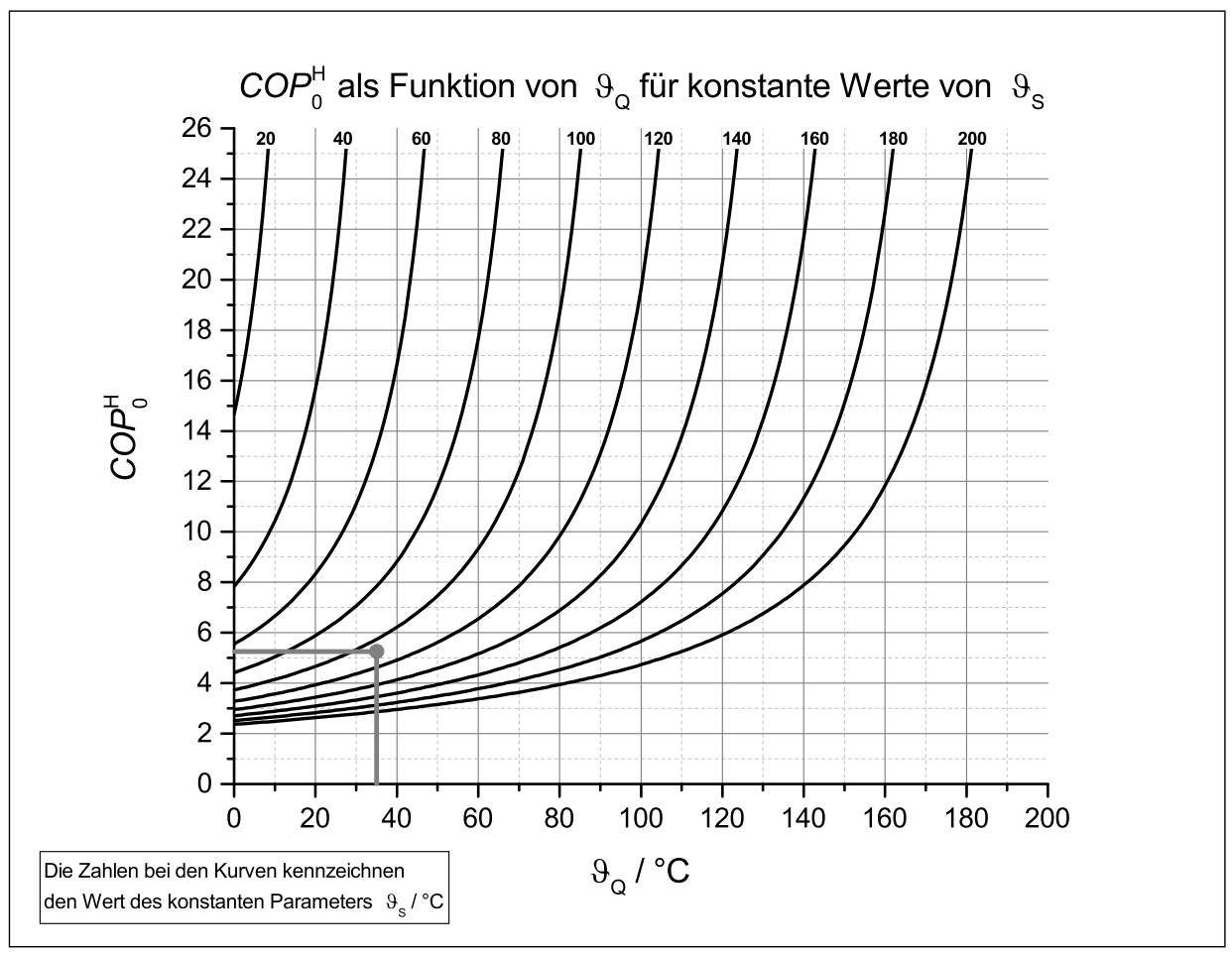

Abbildung 5.2: Fallbeispiel: Ermittlung von $C O P_{0}^{\mathrm{H}}$ 


\section{Ermittlung der dimensionslosen Temperaturdifferenz $\widehat{\Delta T}$}

Zur Bestimmung von $\widehat{\Delta T}$ wird im ersten Schritt die Temperaturdifferenz zwischen Senke und Quelle $\Delta T_{\mathrm{SQ}}$ gebildet. Daran anschließend kann $\widehat{\Delta T}$ aus Abbildung 5.3 unter Einsatz von $\Delta T_{\mathrm{SQ}}$ und der charakteristischen Temperaturdifferenz in den Wärmetauschern $\Delta T_{\mathrm{WT}}$ (vgl. Tabelle 5.1) abgelesen werden:

$$
\begin{aligned}
\Delta T_{\mathrm{SQ}} & =T_{\mathrm{S}}-T_{\mathrm{Q}}=\vartheta_{\mathrm{S}}-\vartheta_{\mathrm{Q}} \\
\Delta T_{\mathrm{SQ}} & =110{ }^{\circ} \mathrm{C}-35^{\circ} \mathrm{C} \\
\rightarrow \Delta T_{\mathrm{SQ}} & =75^{\circ} \mathrm{C} \\
\Delta T_{\mathrm{WT}} & =5{ }^{\circ} \mathrm{C} \\
\Rightarrow \widehat{\Delta T} & =0,065
\end{aligned}
$$

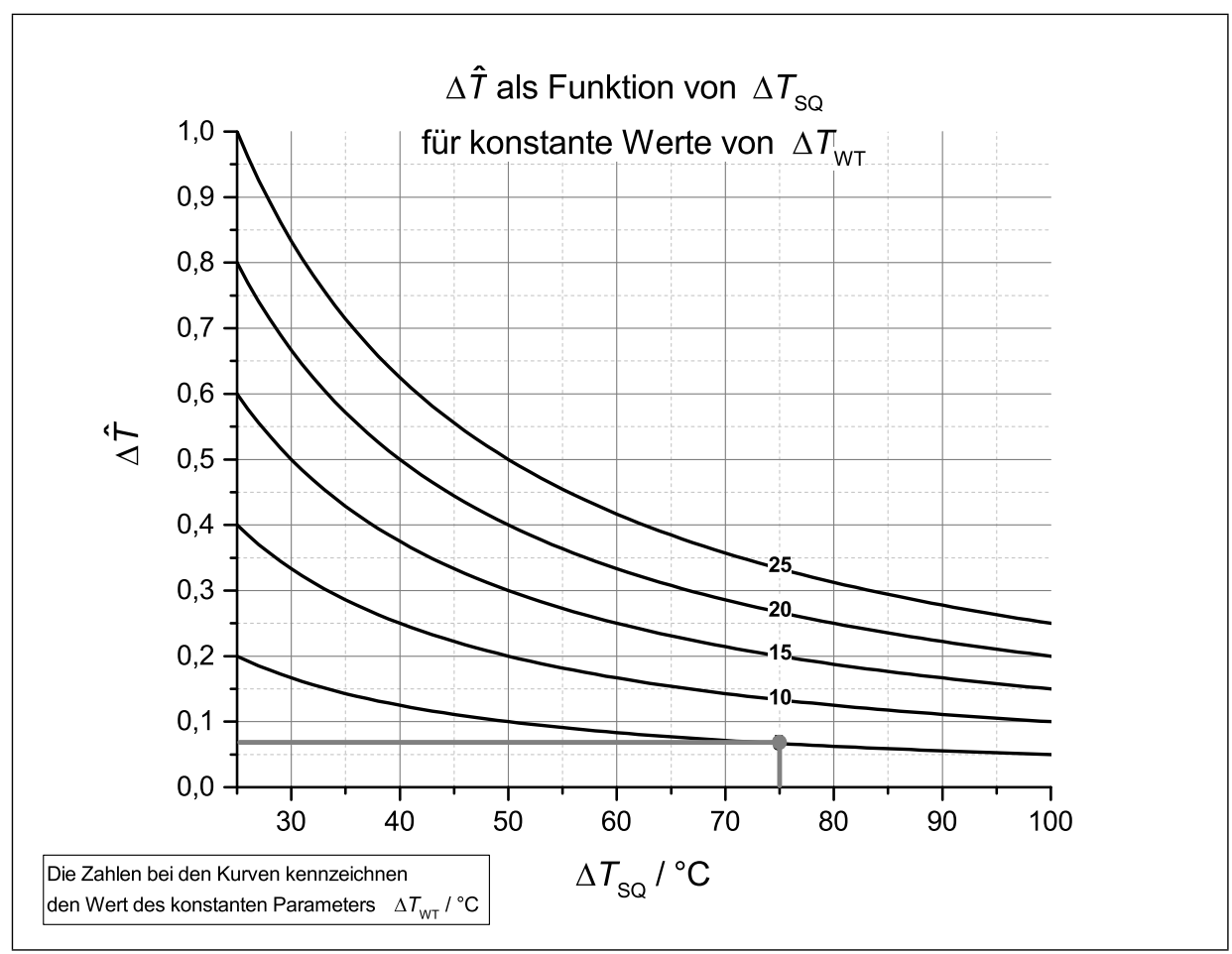

Abbildung 5.3: Fallbeispiel: Ermittlung von $\widehat{\Delta T}$ 


\section{Ermittlung des Temperaturfaktors $\boldsymbol{X}_{\mathrm{T}}$}

Der Temperaturfaktor $X_{\mathrm{T}}$ ist eine Funktion von $C O P_{0}^{\mathrm{H}}$ und $\widehat{\Delta T}$. Daraus folgt unter Verwendung von Abbildung 5.4:

$$
\begin{gathered}
C O P_{0}^{\mathrm{H}}=5,2 \quad \widehat{\Delta T}=0,065 \\
\Rightarrow X_{\mathrm{T}}=0,88
\end{gathered}
$$

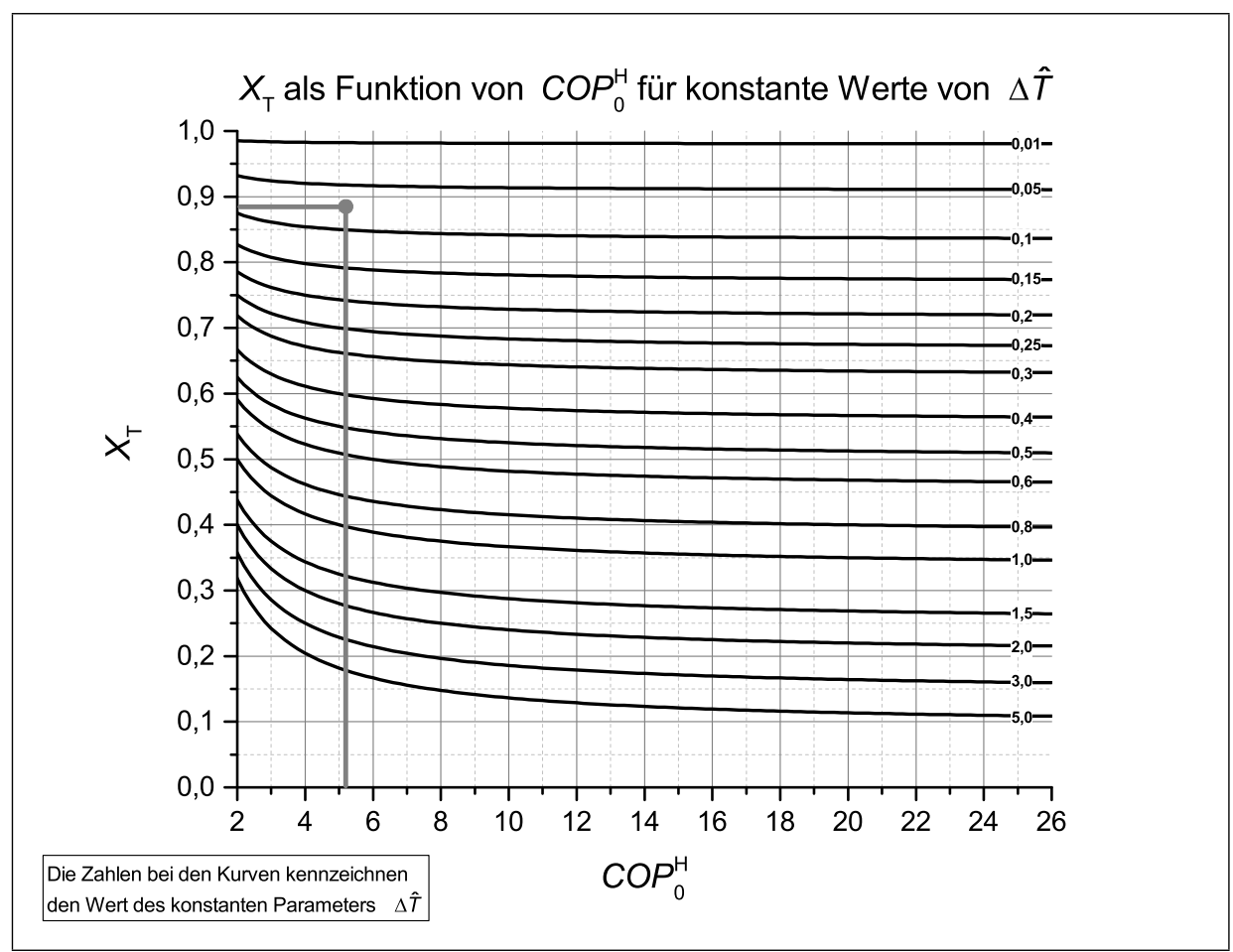

Abbildung 5.4: Fallbeispiel: Ermittlung von $X_{\mathrm{T}}$

\section{Ermittlung des maximalen COPs im Heizbetrieb $C O P_{\max }^{\mathrm{H}}$}

Der maximale COP im Heizbetrieb ist abhängig von dem Temperaturfaktor $X_{\mathrm{T}}$ und der Hilfsgröße $C O P_{0}^{\mathrm{H}}$. Werden die bisher ermittelten Größen verwendet, so lässt sich aus Abbildung 5.5 für $C O P_{\max }^{\mathrm{H}}$ ablesen:

$$
\begin{gathered}
C O P_{0}^{\mathrm{H}}=5,2 \quad X_{\mathrm{T}}=0,88 \\
\Rightarrow C O P_{\max }^{\mathrm{H}}=4,49
\end{gathered}
$$




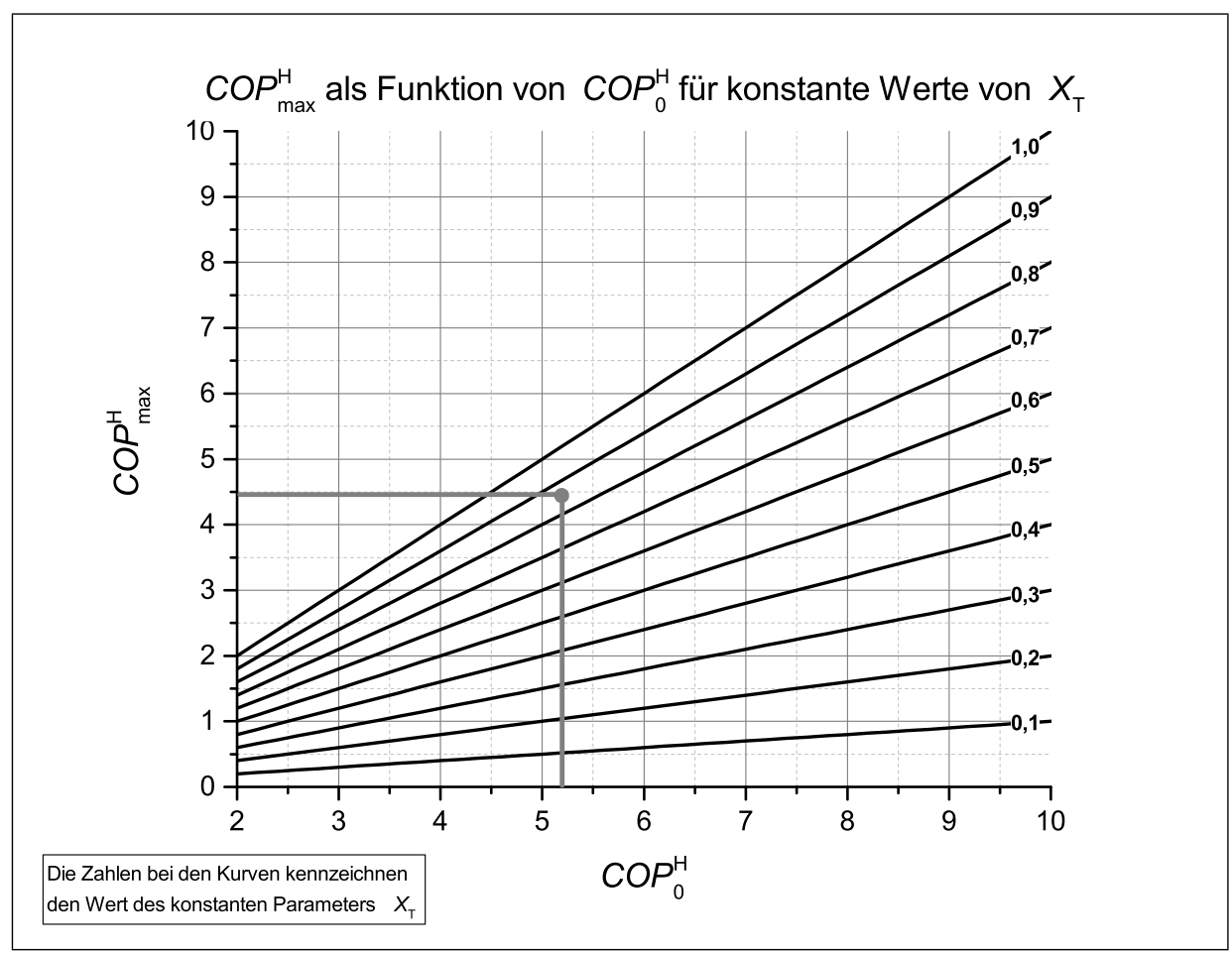

Abbildung 5.5: Fallbeispiel: Ermittlung von $C O P_{\max }^{\mathrm{H}}$

\section{Ermittlung des realen COPs im Heizbetrieb $C O P_{\text {real }}^{\mathrm{H}}$}

Die Bestimmung von $C O P_{\text {real }}^{\mathrm{H}}$ aus Abbildung 5.6 mit dem Gütegrad aus Tabelle 5.1 und $C O P_{\max }^{\mathrm{H}}$ ergibt:

$$
\begin{gathered}
C O P_{\max }^{\mathrm{H}}=4,49 \quad \eta_{\mathrm{WP}}=0,5 \\
\Rightarrow C O P_{\text {real }}^{\mathrm{H}}=2,2
\end{gathered}
$$

Hinweis: Die Bestimmung von $C O P_{\text {real }}^{\mathrm{H}}$ ist an dieser Stelle sinnvoll, jedoch nur dann notwendig, wenn der Quotient der Nutzwärmeströme $\widehat{Q}$ daraus ermittelt werden soll. Für die relative Energiekosteneinsparung im Heizbetrieb wird $C O P$ real jedoch nicht benötigt. 


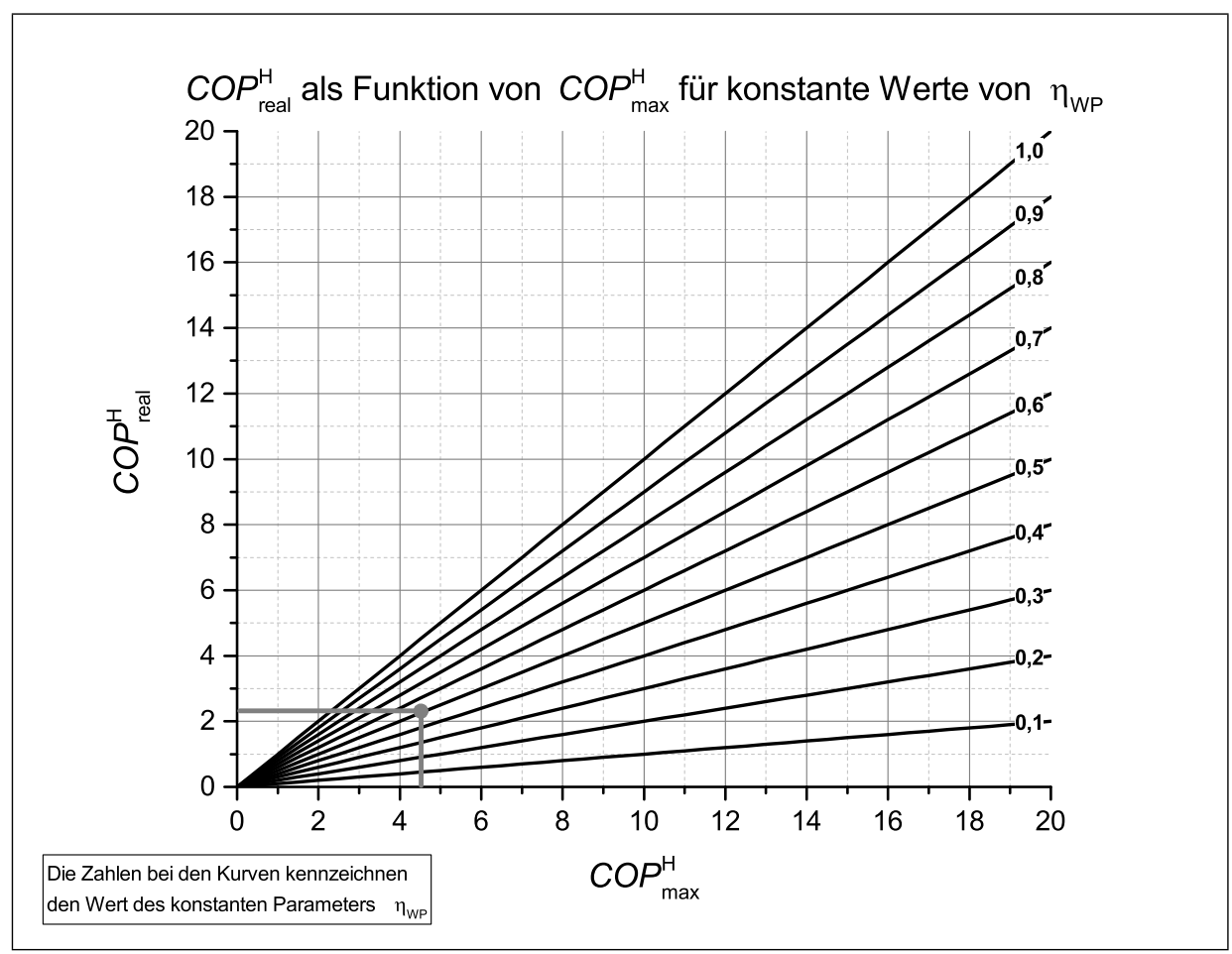

Abbildung 5.6: Fallbeispiel: Ermittlung von $C O P_{\text {real }}^{\mathrm{H}}$

\section{Ermittlung des Gesamteinflussfaktors im Heizbetrieb $\boldsymbol{F}_{\text {ges }}^{\mathbf{H}}$}

Zur Bestimmung von $F_{\text {ges }}^{\mathrm{H}}$ sind zwei Schritte notwendig. Im ersten Schritt wird das Verhältnis von $\eta_{\mathrm{B}}$ zu $\eta_{\mathrm{A}}$ aus Abbildung 5.7 abgelesen. Im zweiten Schritt kann, nach Bildung des Quotienten aus den spezifischen Kosten $K_{\mathrm{A}}$ und $K_{\mathrm{B}}, F_{\text {ges }}^{\mathrm{H}}$ unter Verwendung von Abbildung 5.8 bestimmt werden:

$$
\begin{gathered}
\eta_{\mathrm{A}}=\eta_{\mathrm{WP}}=0,5 \quad \eta_{\mathrm{B}}=\eta_{\mathrm{kon}}^{\mathrm{H}}=0,85 \\
\Rightarrow \frac{\eta_{\mathrm{B}}}{\eta_{\mathrm{A}}}=\frac{\eta_{\mathrm{kon}}^{\mathrm{H}}}{\eta_{\mathrm{WP}}}=1,7 \\
K_{\mathrm{WP}}^{\mathrm{H}}=K_{\mathrm{el}}=€ 60 / \mathrm{MWh} \quad K_{\mathrm{kon}}^{\mathrm{H}}=€ 25 / \mathrm{MWh} \\
\frac{K_{\mathrm{A}}}{K_{\mathrm{B}}}=\frac{K_{\mathrm{WP}}^{\mathrm{H}}}{K_{\mathrm{kon}}^{\mathrm{H}}}=\frac{€ 60 / \mathrm{MWh}}{€ 25 / \mathrm{MWh}} \\
\rightarrow \frac{K_{\mathrm{A}}}{K_{\mathrm{B}}}=2,4 \\
\Rightarrow F_{\text {ges }}^{\mathrm{H}}=4,2
\end{gathered}
$$




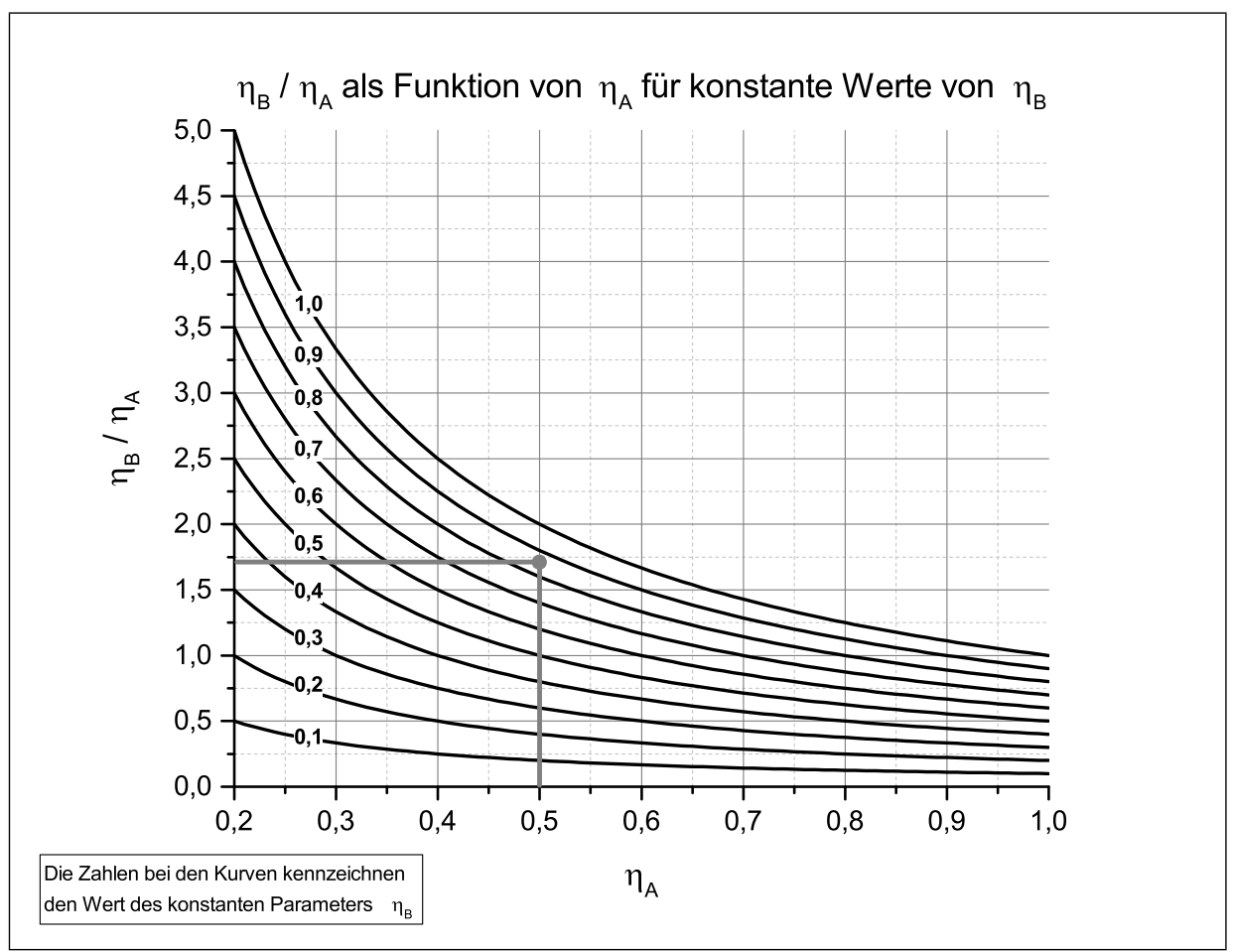

Abbildung 5.7: Fallbeispiel: Ermittlung von $\eta_{\mathrm{B}} \mathrm{zu} \eta_{\mathrm{A}}$

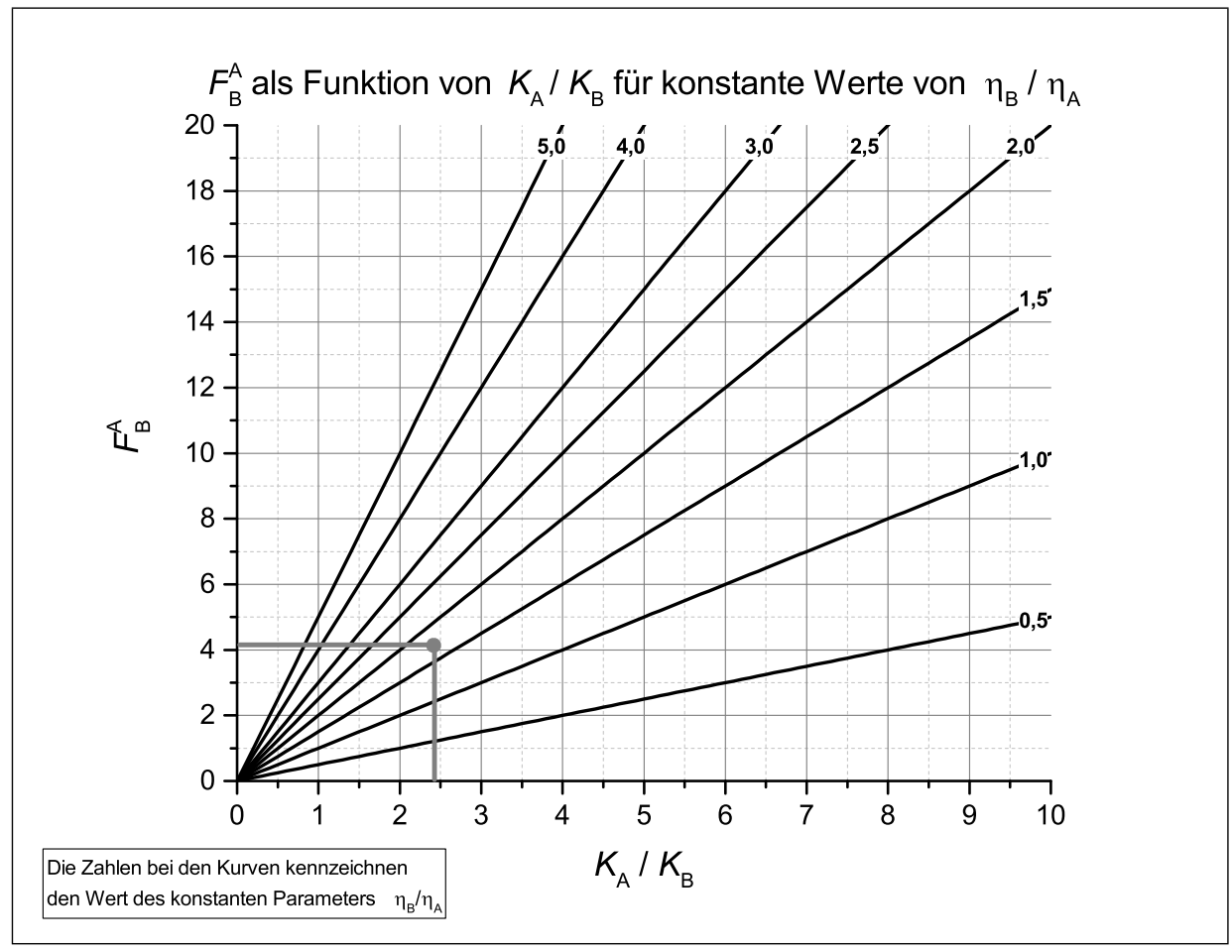

Abbildung 5.8: Fallbeispiel: Ermittlung von $F_{\text {ges }}^{\mathrm{H}}$ 
Ermittlung der relativen Energiekosteneinsparung im Heizbetrieb $\widehat{\boldsymbol{E}}^{\mathbf{H}}$

Abbildung 5.9 zeigt die relative Energiekosteneinsparung im Heizbetrieb, welche unter Verwendung der ermittelten Parametern ablesbar ist:

$$
\begin{gathered}
C O P_{\max }^{\mathrm{H}}=4,49 \quad F_{\text {ges }}^{\mathrm{H}}=4,2 \\
\Rightarrow \widehat{E}^{\mathrm{H}}=0,055
\end{gathered}
$$

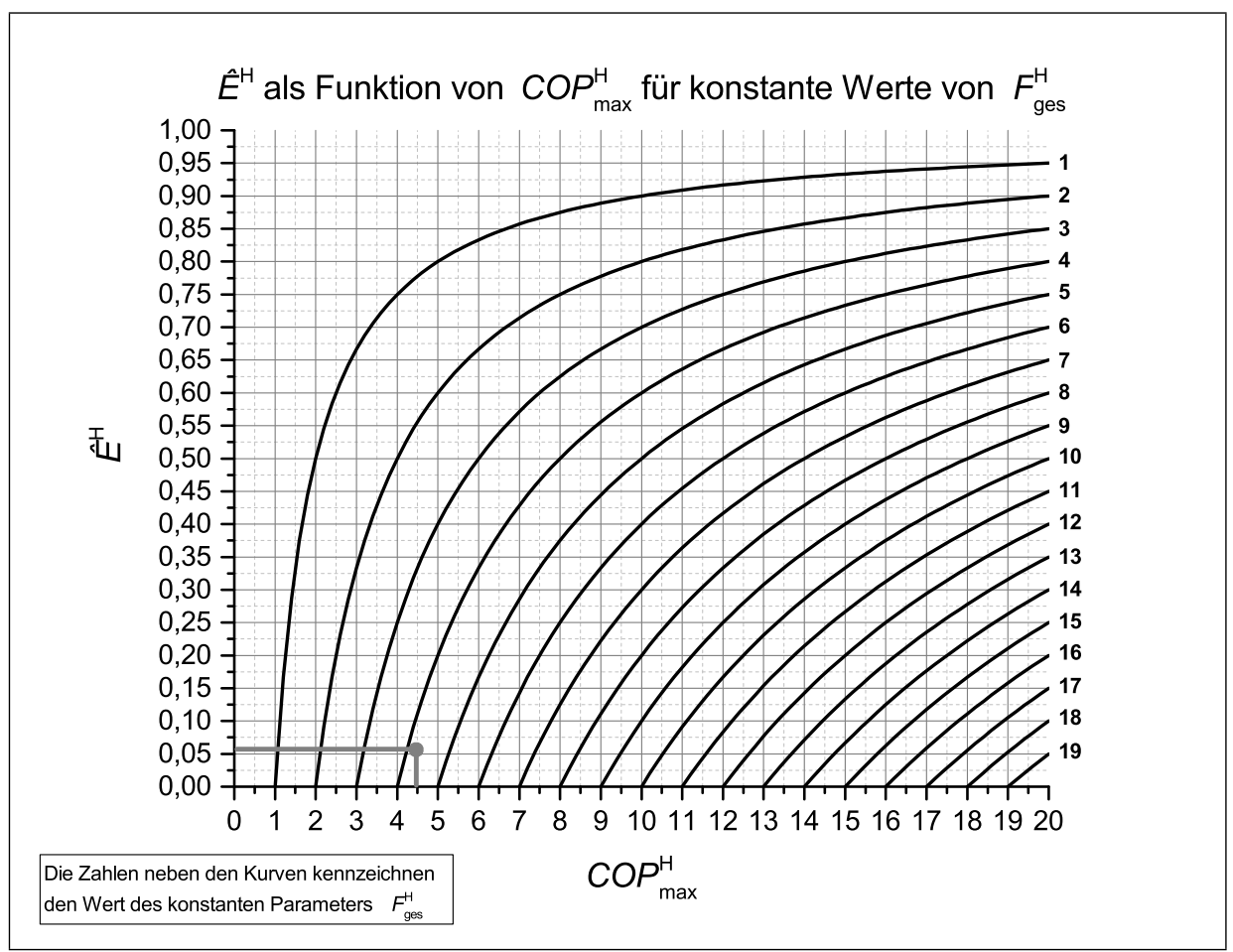

Abbildung 5.9: Fallbeispiel: Ermittlung von $\widehat{E}^{\mathrm{H}}$

\section{Fazit zur Bereitstellung Sattdampf bei $110^{\circ} \mathrm{C}$}

Auf dem Ableseweg wurde ermittelt:

$$
\Rightarrow \widehat{E}^{\mathrm{H}}=0,055
$$

Rechnerisch ergibt sich:

$$
\widehat{E}^{\mathrm{H}}=0,10653
$$

Der verhältnismäßig große Unterschied ist auf die hohen Änderungsraten von $\widehat{E}^{\mathrm{H}}$ bei kleinen Werten von $C O P_{\text {max }}^{\mathrm{H}}$ und $F_{\text {ges }}^{\mathrm{H}}$ zurückzuführen. 
Für das Beispiel folgt:

Die ermittelte relative Energiekosteneinsparung im Heizbetrieb ist aus Sicht des Unternehmens nicht zufriedenstellend. Um den Einsatz einer Wärmepumpe überhaupt in Betracht zu ziehen, muss zumindest eine relative Einsparung im Heizbetrieb von $25 \%$ erzielbar sein. Da im Unternehmen Wärmesenken auf verschiedenen Temperaturniveaus vorhanden sind, ist es nicht zweckmäßig, alle einzeln einer Bewertung zu unterziehen. Es gilt vielmehr die maximale Wärmenutzungstemperatur, die gerade noch $\widehat{E}^{\mathrm{H}}=0,25$ erfüllt, zu ermitteln.

\subsection{Inverse Bestimmung von $\vartheta_{\mathrm{S}}^{\max }$ bei gegebenem $\widehat{\boldsymbol{E}}^{\mathrm{H}}$}

Das Ziel der Analyse ist die Bestimmung der maximalen Wärmenutzungstemperatur $\vartheta_{\mathrm{S}}^{\max }$ bei gegebener relativer Energiekosteneinsparung im Heizbetrieb $\widehat{E}^{\mathrm{H}}=0,25$. Nachdem Gesamteinflussfaktor im Heizbetrieb $F_{\text {ges }}^{\mathrm{H}}$ nicht von der Temperatur der Quelle und der Senke abhängt und weiterhin die Randbedingungen nach Tabelle 5.1 gelten, wird bei der Bearbeitung der inverse Lösungswegs vollzogen:

1. Ermittlung des erforderlichen maximalen COPs im Heizbetrieb $C O P_{\max }^{\mathrm{H}}$.

2. Bestimmung der maximalen Temperatur der Senke $\vartheta_{\mathrm{S}}^{\max }$.

Zur Bestimmung des minimalen Werts von $C O P_{\max }^{\mathrm{H}}$ bei $\widehat{E}^{\mathrm{H}}=0,25$ kann, unter Verwendung des bereits bestimmten Werts für $F_{\text {ges }}^{\mathrm{H}}$, aus Abbildung 5.10 abgelesen werden:

$$
\begin{gathered}
\widehat{E}^{\mathrm{H}}=0,25 \quad F_{\mathrm{ges}}^{\mathrm{H}}=4,2 \\
\Rightarrow C O P_{\max }^{\mathrm{H}}=5,8
\end{gathered}
$$




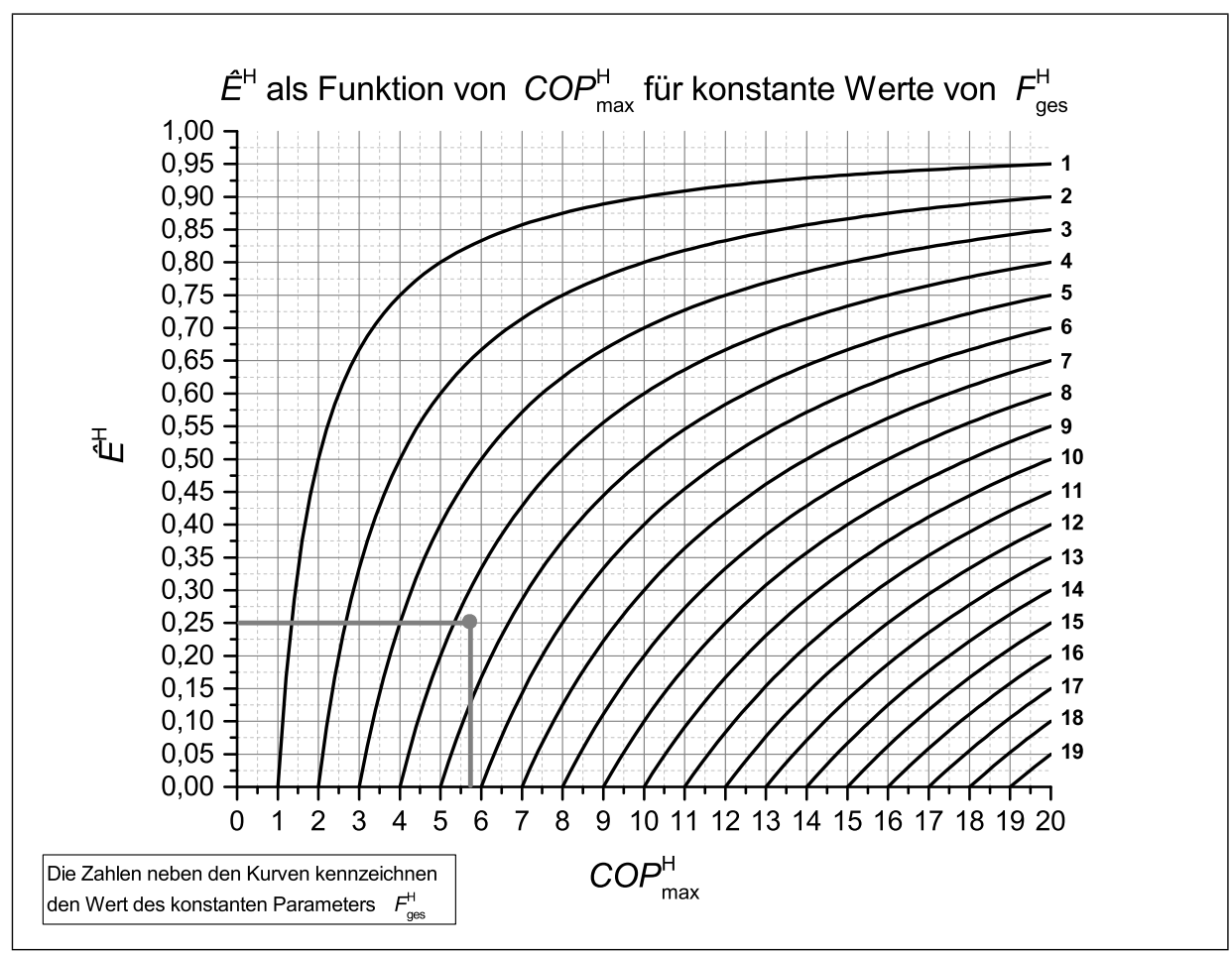

Abbildung 5.10: Fallbeispiel: Ermittlung des erforderlichen Werts von $C O P_{\max }^{\mathrm{H}}$

Um $\vartheta_{\mathrm{S}}^{\max } \mathrm{zu}$ ermitteln, kann grundsätzlich das direkte Schema unter Verwendung von $X_{\mathrm{T}}$ zum Einsatz kommen. Hierbei müssten jedoch ggf. mehrere Schritte durchgeführt werden. Zweckmäßiger ist an dieser Stelle daher die Verwendung des gemittelten maximalen COPs

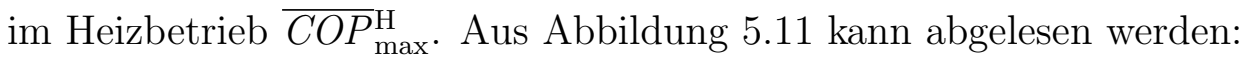

$$
\begin{aligned}
& \overline{C O P}_{\max }^{\mathrm{H}}=C O P_{\max }^{\mathrm{H}} \\
& \overline{C O P}_{\max }^{\mathrm{H}}=5,8 \quad \vartheta_{\mathrm{Q}}=35{ }^{\circ} \mathrm{C} \\
& \Rightarrow \vartheta_{\mathrm{S}}^{1}=60{ }^{\circ} \mathrm{C} \text { bei } \Delta T_{\mathrm{WT}}^{1}=20{ }^{\circ} \mathrm{C}
\end{aligned}
$$




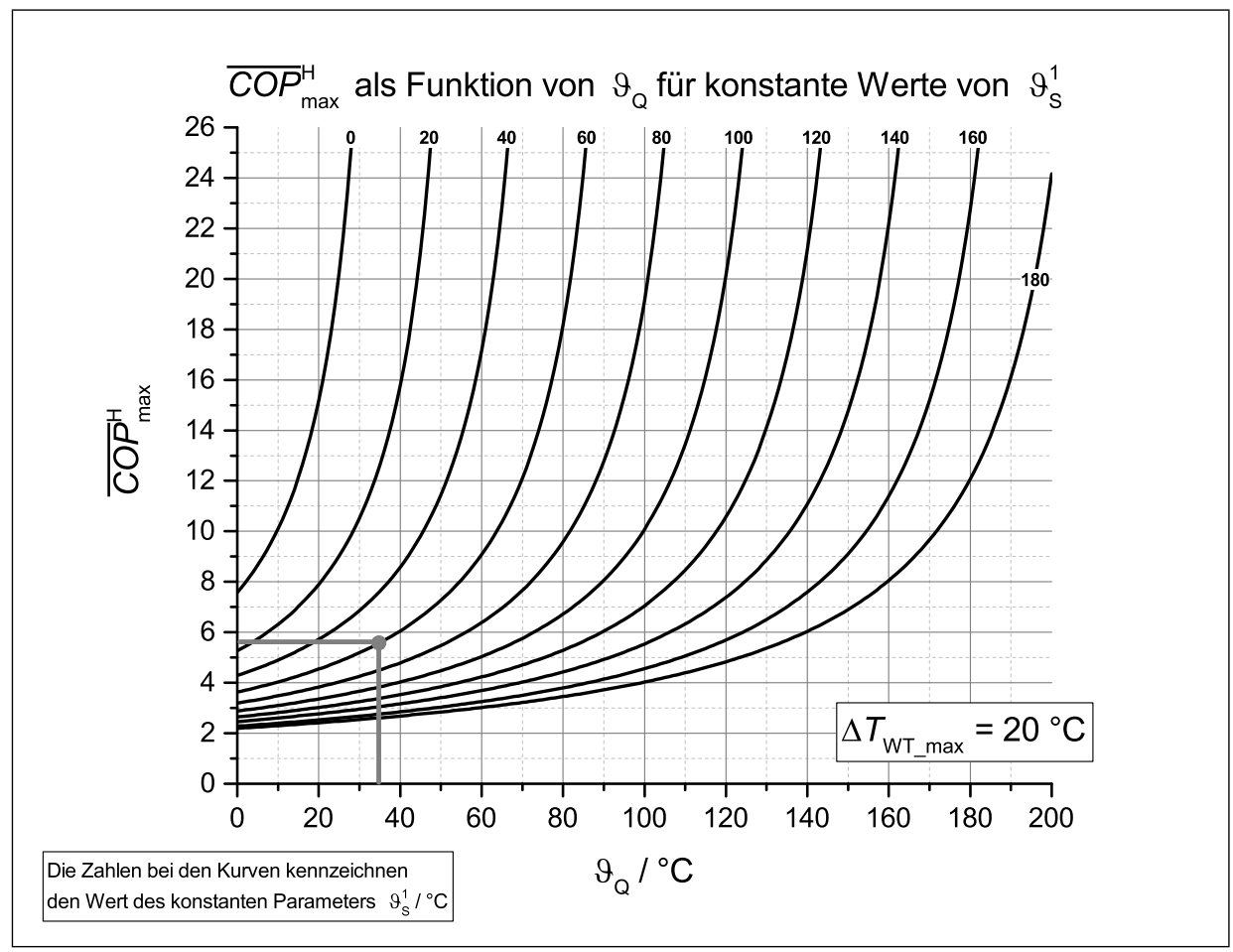

Abbildung 5.11: Fallbeispiel: Ermittlung von $\overline{C O P}_{\max }^{\mathrm{H}}$

Für die ermittelten Werte von $\vartheta_{\mathrm{S}}^{1}$ bei $\Delta T_{\mathrm{WT}}^{1}$ kann $\vartheta_{\mathrm{S}}^{\max }$ bei $\Delta T_{\mathrm{WT}}=5{ }^{\circ} \mathrm{C}$ aus Tabelle 5.2 bestimmt werden. Die betreffende Zeile ist grau hervorgehoben. Daraus folgt:

$$
\begin{gathered}
\vartheta_{\mathrm{S}}^{1}=60{ }^{\circ} \mathrm{C} \text { bei } \Delta T_{\mathrm{WT}}^{1}=20^{\circ} \mathrm{C} \\
\Rightarrow \vartheta_{\mathrm{S}}^{\max }=90{ }^{\circ} \mathrm{C} \text { bei } \Delta T_{\mathrm{WT}}=5^{\circ} \mathrm{C}
\end{gathered}
$$

Tabelle 5.2: Fallbeispiel: Verwendung von Tabelle 3.1

\begin{tabular}{rrrrrrr}
\hline & $A=0$ & $A=10$ & $A=20$ & $A=30$ & $A=40$ & \\
\hline$\vartheta_{\mathrm{S}}^{1} \mid \Delta T_{\mathrm{WT}}^{1}$ & $\vartheta_{\mathrm{S}}^{2} \mid \Delta T_{\mathrm{WT}}^{2}$ & $\vartheta_{\mathrm{S}}^{2} \mid \Delta T_{\mathrm{WT}}^{2}$ & $\vartheta_{\mathrm{S}}^{2} \mid \Delta T_{\mathrm{WT}}^{2}$ & $\vartheta_{\mathrm{S}}^{2} \mid \Delta T_{\mathrm{WT}}^{2}$ & $\vartheta_{\mathrm{S}}^{2} \mid \Delta T_{\mathrm{WT}}^{2}$ & $\delta_{\max }$ \\
\hline $180 \mid 20$ & $180 \mid 20$ & $190 \mid 15$ & $200 \mid 10$ & $210 \mid 5$ & $220 \mid 0$ & 0,021135 \\
$160 \mid 20$ & $160 \mid 20$ & $170 \mid 15$ & $180 \mid 10$ & $190 \mid 5$ & $200 \mid 0$ & 0,022068 \\
$140 \mid 20$ & $140 \mid 20$ & $150 \mid 15$ & $160 \mid 10$ & $170 \mid 5$ & $180 \mid 0$ & 0,023087 \\
$120 \mid 20$ & $120 \mid 20$ & $130 \mid 15$ & $140 \mid 10$ & $150 \mid 5$ & $160 \mid 0$ & 0,024204 \\
$100 \mid 20$ & $100 \mid 20$ & $110 \mid 15$ & $120 \mid 10$ & $130 \mid 5$ & $140 \mid 0$ & 0,025436 \\
$80 \mid 20$ & $80 \mid 20$ & $90 \mid 15$ & $100 \mid 10$ & $110 \mid 5$ & $120 \mid 0$ & 0,026799 \\
$60 \mid 20$ & $60 \mid 20$ & $70 \mid 15$ & $80 \mid 10$ & $90 \mid 5$ & $100 \mid 0$ & 0,028317 \\
$40 \mid 20$ & $40 \mid 20$ & $50 \mid 15$ & $60 \mid 10$ & $70 \mid 5$ & $80 \mid 0$ & 0,030017 \\
$20 \mid 20$ & $20 \mid 20$ & $30 \mid 15$ & $40 \mid 10$ & $50 \mid 5$ & $60 \mid 0$ & 0,031934 \\
$0 \mid 20$ & $0 \mid 20$ & $10 \mid 15$ & $20 \mid 10$ & $30 \mid 5$ & $40 \mid 0$ & 0,034112 \\
\hline
\end{tabular}


Fazit zur Ermittlung der maximalen Wärmenutzungstemperatur bei $\widehat{\boldsymbol{E}}^{\mathbf{H}}=\mathbf{0 , 2 5}$ Wenn die Temperatur der Senke $90{ }^{\circ} \mathrm{C}$ nicht überschreitet, so kann im Heizbetrieb mit einer relativen Energiekosteneinsparung von mindestens $25 \%$ gerechnet werden.

Rechnerisch lässt sich ermitteln, dass unter den gegebenen Randbedingungen ein $\vartheta_{\mathrm{S}}=$ $93,277^{\circ} \mathrm{C} z u \widehat{E}^{\mathrm{H}}=0,25$ führt. Das graphische Schema liefert in diesem Fall daher sehr gute Ergebnisse.

Für das Beispiel folgt:

Das höchste Temperaturniveau der Wärmeversorgung, das sich unter $\vartheta_{\mathrm{S}}^{\max }=90^{\circ} \mathrm{C}$ befindet, ist jenes bei $85^{\circ} \mathrm{C}$. Das Unternehmen wünscht sich für diesen Fall die folgenden Abschätzungen:

1. Wie viel würde eine Wärmepumpe bei vollständiger Ausnutzung der Leistung der Quelle kosten?

2. Wie viele Jahre müsste eine derartige Wärmepumpe unter konstanten betrieblichen Rahmenbedingungen in Betrieb sein, damit sich diese amortisiert hätte?

\subsection{Direkte Ermittlung von $E\left(\Delta t_{\mathrm{P}}\right)$ und $\Delta t_{\mathrm{A}}$ im Heiz- betrieb}

Um die absolute Einsparung im Betrachtungszeitraum $E\left(\Delta t_{\mathrm{P}}\right)$ sowie die Amortisationsdauer $\Delta t_{\mathrm{A}}$ auf dem direkten Weg ermitteln zu können, sind mehrere Schritte notwendig.

Die Bestimmung von $\widehat{E}^{\mathrm{H}}$ bei den gegebenen Randbedingungen und $\vartheta_{\mathrm{S}}=85{ }^{\circ} \mathrm{C}$ ist bereits im Detail vorgestellt worden. Um die Anzahl der Abbildungen nicht unnötig in die Höhe zu treiben, werden im Folgenden kurz die Resultate aus der Ablesung aufgeführt, bevor die absoluten wirtschaftlichen Kennzahlen im Detail errechnet werden.

Bestimmung der relativen Energiekosteneinsparung im Heizbetrieb $\widehat{\boldsymbol{E}}^{\mathbf{H}}$ Aus den bekannten Diagrammen folgt für die Ermittlung von $\widehat{E}^{\mathrm{H}}$ :

- Ermittlung des maximalen COPs im Heizbetrieb bei $\Delta T_{\mathrm{WT}}=0{ }^{\circ} \mathrm{C}$ :

$$
\begin{aligned}
\vartheta_{\mathrm{S}} & =85{ }^{\circ} \mathrm{C} \quad \vartheta_{\mathrm{Q}}=35{ }^{\circ} \mathrm{C} \\
& \Rightarrow C O P_{0}^{\mathrm{H}}=7,1
\end{aligned}
$$


- Ermittlung der dimensionslosen Temperaturdifferenz $\widehat{\Delta T}$ :

$$
\begin{aligned}
\Delta T_{\mathrm{SQ}} & =T_{\mathrm{S}}-T_{\mathrm{Q}}=\vartheta_{\mathrm{S}}-\vartheta_{\mathrm{Q}} \\
\Delta T_{\mathrm{SQ}} & =85^{\circ} \mathrm{C}-35^{\circ} \mathrm{C} \\
\rightarrow \Delta T_{\mathrm{SQ}} & =50^{\circ} \mathrm{C} \\
\Delta T_{\mathrm{WT}} & =5{ }^{\circ} \mathrm{C} \\
\Rightarrow \widehat{\Delta T} & =0,1
\end{aligned}
$$

- Ermittlung des Temperaturfaktors $X_{\mathrm{T}}$ :

$$
\begin{gathered}
C O P_{0}^{\mathrm{H}}=7,1 \quad \widehat{\Delta T}=0,1 \\
\Rightarrow X_{\mathrm{T}}=0,84
\end{gathered}
$$

- Ermittlung des maximalen COPs im Heizbetrieb $C O P_{\max }^{\mathrm{H}}$ :

$$
\begin{gathered}
C O P_{0}^{\mathrm{H}}=7,1 \quad X_{\mathrm{T}}=0,84 \\
\Rightarrow C O P_{\max }^{\mathrm{H}}=6,0
\end{gathered}
$$

- Ermittlung des realen COPs im Heizbetrieb $C O P_{\text {real }}^{\mathrm{H}}$ :

$$
\begin{gathered}
C O P_{\max }^{\mathrm{H}}=6,0 \quad \eta_{\mathrm{WP}}=0,5 \\
\Rightarrow C O P_{\text {real }}^{\mathrm{H}}=3,0
\end{gathered}
$$

- Der Gesamteinflussfaktor im Heizbetrieb $F_{\text {ges }}^{\mathrm{H}}$ hat sich nicht geändert:

$$
\Rightarrow F_{\mathrm{ges}}^{\mathrm{H}}=4,2
$$

- Ermittlung der relativen Energiekosteneinsparung im Heizbetrieb $\widehat{E}^{\mathrm{H}}$ :

$$
\begin{gathered}
C O P_{\max }^{\mathrm{H}}=6,0 \quad F_{\text {ges }}^{\mathrm{H}}=4,2 \\
\Rightarrow \widehat{E}^{\mathrm{H}}=0,31
\end{gathered}
$$




\section{Ermittlung des Quotienten der Nutzwärmeströme $\widehat{\boldsymbol{Q}}$}

Der Quotient der Nutzwärmeströme wird im Heizbetrieb dann benötigt, wenn die Leistung der Quelle und nicht die der Senke bekannt ist. Aus Abbildung 5.12 lässt sich ablesen:

$$
\begin{aligned}
& C O P_{\text {real }}^{\mathrm{H}}=3 \\
& \Rightarrow \widehat{Q}=0,67
\end{aligned}
$$

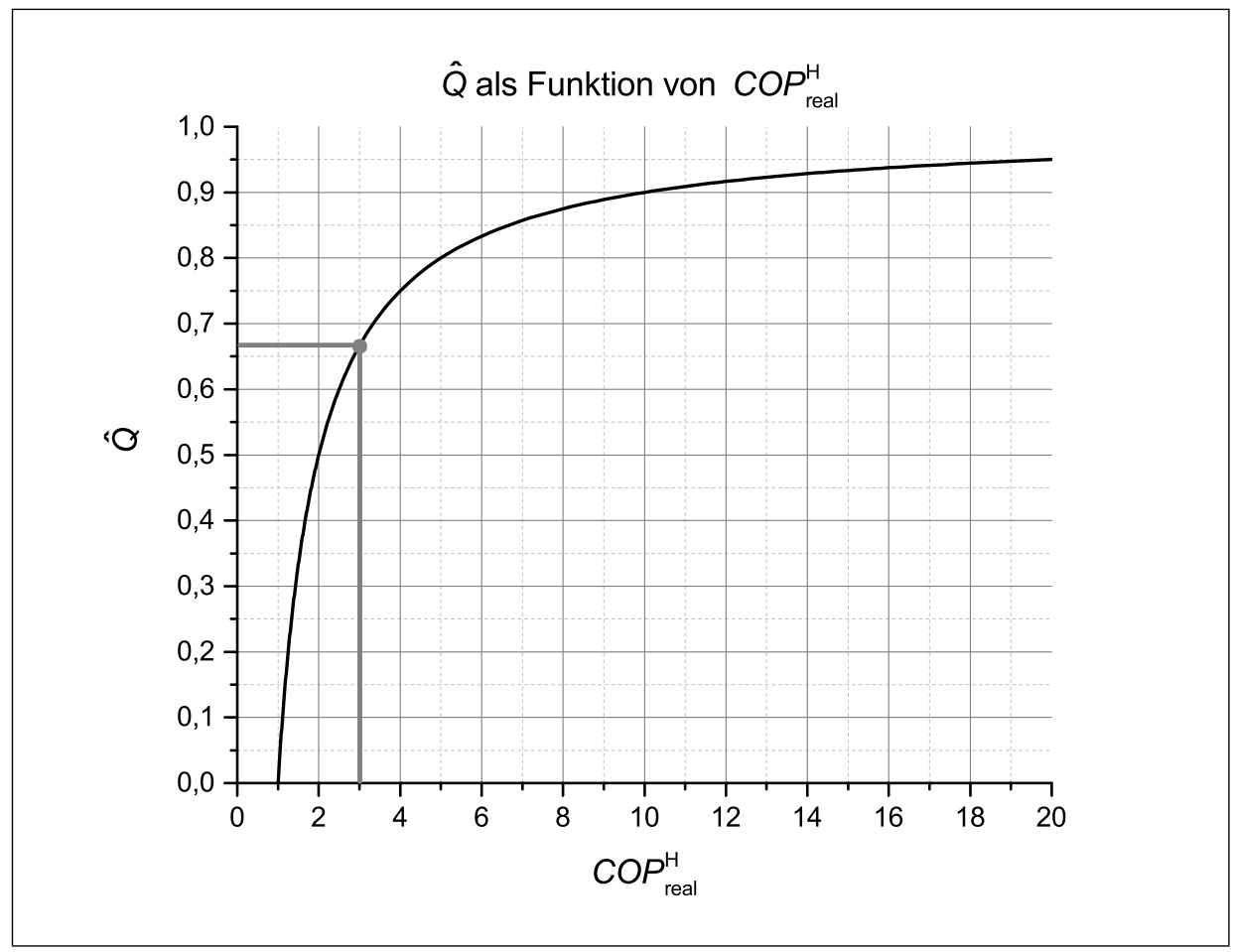

Abbildung 5.12: Fallbeispiel: Ermittlung von $\widehat{Q}$

Daraus lässt sich die reale Leistung an der Senke $\dot{Q}_{\mathrm{N}}^{\mathrm{K}}$ errechnen:

$$
\begin{aligned}
\widehat{Q} & =0,67 \\
\widehat{Q} & =\frac{\dot{Q}_{\mathrm{N}}^{\mathrm{K}}}{\dot{Q}_{\mathrm{N}}^{\mathrm{H}}} \\
\dot{Q}_{\mathrm{N}}^{\mathrm{H}} & =\frac{\dot{Q}_{\mathrm{N}}^{\mathrm{K}}}{\widehat{Q}}=\frac{850 \mathrm{~kW}}{0,67} \\
\rightarrow \dot{Q}_{\mathrm{N}}^{\mathrm{H}} & =1.268,66 \mathrm{~kW}
\end{aligned}
$$




\section{Ermittlung der Investitionskosten $I K_{\mathrm{WP}}$}

Zur Berechnung der Investitionskosten $I K_{\mathrm{WP}}$, wird der Mittelwert der spezifischen von der Heizleistung abhängigen Kosten $K_{\mathrm{WP}}^{\mathrm{IK}}$ nach Kapitel 3.5.3 gebildet:

$$
\begin{aligned}
K_{\mathrm{WP}}^{\mathrm{IK}} & =\frac{1}{2}\left(\frac{€ 250}{\mathrm{~kW}}+\frac{€ 400}{\mathrm{~kW}}\right) \\
& \rightarrow K_{\mathrm{WP}}^{\mathrm{IK}}=\frac{€ 325}{\mathrm{~kW}}
\end{aligned}
$$

und mit dem in Gleichung (5.3) ermittelten Wert für $\dot{Q}_{\mathrm{N}}^{\mathrm{K}}$ multipliziert:

$$
\begin{gathered}
I K_{\mathrm{WP}}=\dot{Q}_{\mathrm{N}}^{\mathrm{H}} K_{\mathrm{WP}}^{\mathrm{IK}} \quad\left[\dot{Q}_{\mathrm{N}}^{\mathrm{H}}\right]=\mathrm{kW} \\
I K_{\mathrm{WP}}=\frac{€ 325}{\mathrm{~kW}} \cdot 1.268,66 \mathrm{~kW} \\
\rightarrow I K_{\mathrm{WP}}=€ 412.313,43
\end{gathered}
$$

wodurch die Investitionskosten bestimmt sind. 


\section{Ermittlung der absoluten Einsparung im Betrachtungszeitraum $E\left(\Delta t_{\mathrm{P}}\right)$}

Für die Amortisationsdauer $\Delta t_{\mathrm{A}}$ gilt, dass unter den gegebenen Bedingungen nur ein Betriebspunkt zu berücksichtigen ist. Daraus folgt:

$$
\Delta t_{\mathrm{P}}=\Delta t_{\mathrm{i}}=8.760 \mathrm{~h}
$$

was, bei Verwendung des Resultats aus Gleichung (5.3), in das Schema aus Kapitel 3.5.1 eingesetzt:

$$
\begin{gathered}
Q_{\mathrm{N}}^{\mathrm{H}}\left(P_{\mathrm{t}}\right)=\Delta t_{\mathrm{P}} \dot{Q}_{\mathrm{N}}^{\mathrm{H}}\left(P_{\mathrm{t}}\right)=8.760 \mathrm{~h} \cdot 1.268,66 \mathrm{~kW} \\
\rightarrow Q_{\mathrm{N}}^{\mathrm{H}}\left(P_{\mathrm{t}}\right)=11.113 .433 \mathrm{kWh}=11.113,43 \mathrm{MWh} \\
K_{\mathrm{kon}}^{\mathrm{H}}=€ 25 / \mathrm{MWh} \\
G K_{\mathrm{kon}}^{\mathrm{H}}\left(P_{\mathrm{i}}\right)=G K_{\mathrm{kon}}^{\mathrm{H}}\left(P_{\mathrm{t}}\right)=\frac{Q_{\mathrm{N}}^{\mathrm{H}}}{\eta_{\mathrm{kon}}^{\mathrm{H}}} K_{\mathrm{kon}}^{\mathrm{H}}=\frac{11.113,43 \mathrm{MWh}}{0,85} \cdot € 25 / \mathrm{MWh} \\
\rightarrow G K_{\mathrm{kon}}^{\mathrm{H}}\left(P_{\mathrm{t}}\right)=€ 326.865,67 \\
E\left(\Delta t_{\mathrm{P}}\right)=G K_{\mathrm{kon}}^{\mathrm{H}}\left(P_{\mathrm{t}}\right) \widehat{E}^{\mathrm{H}}\left(P_{\mathrm{t}}\right)=€ 326.865,67 \cdot 0,31 \\
\rightarrow E\left(\Delta t_{\mathrm{P}}\right)=€ 101.328,36
\end{gathered}
$$

die absolute Einsparung im Zeitraum $\Delta t_{\mathrm{P}}$ ergibt.

\section{Ermittlung der Amortisationsdauer $\Delta t_{\mathrm{A}}$}

Die Amortisationsdauer $\Delta t_{\mathrm{A}}$ lässt sich dadurch ermitteln, dass die Investitionskosten aus Gleichung (5.4) mit der absoluten Einsparung aus Gleichung (5.5) normiert werden:

$$
\begin{gathered}
\Delta t_{\mathrm{A}}=\Delta t_{\mathrm{P}} \frac{I K_{\mathrm{WP}}}{E\left(\Delta t_{\mathrm{P}}\right)}=8760 \mathrm{~h} \cdot \frac{€ 412.313,43}{€ 101.328,36} \\
\rightarrow \Delta t_{\mathrm{A}}=35645,16 \mathrm{~h}
\end{gathered}
$$

Wird $\Delta t_{\mathrm{A}}$ in Jahren ausgedrückt, so lässt sich ermitteln:

$$
\Delta t_{\mathrm{A}}=4,07 \text { Jahre }
$$

Werden die graphisch ermittelbaren technischen und wirtschaftlichen Kennzahlen auf dem Rechenweg bestimmt, so ergibt sich eine Amortisationsdauer von:

$$
\Delta t_{\mathrm{A}}=3,87 \text { Jahre }
$$


Für das Beispiel folgt:

Der Einsatz einer Wärmepumpe hätte sich unter den gegebenen Bedingungen in ungefähr 4 Jahren amortisiert. Aus Sicht des Unternehmens stellt sich die Frage, wie sich die Amortisationsdauer verändert, wenn die spezifischen Kosten der Vergleichstechnologie um 10 \% fallen und die der Wärmepumpe um $10 \%$ steigen.

\subsection{Sensitivität der absoluten wirtschaftlichen Kenn- zahlen}

Wenn sich die spezifischen Kosten ändern, so verändern sich nur die wirtschaftlichen Kennzahlen. Da die Ermittlung der wirtschaftlichen Kennzahlen bereits vorgestellt wurde, erfolgt die Dokumentation des Rechenwegs zum Einfluss der spezifischen Kosten in gekürzter Form. Es werden auch nur die Kennzahlen neu ermittelt, die sich tatsächlich ändern.

\section{Bestimmung der relativen Energiekosteneinsparung im Heizbetrieb $\widehat{\boldsymbol{E}}^{\mathbf{H}}$}

- Ermittlung des Gesamteinflussfaktors im Heizbetrieb $F_{\text {ges }}^{\mathrm{H}}$ :

$$
\begin{gathered}
\Rightarrow \frac{\eta_{\mathrm{B}}}{\eta_{\mathrm{A}}}=\frac{\eta_{\mathrm{kon}}^{\mathrm{H}}}{\eta_{\mathrm{WP}}}=1,7 \\
K_{\mathrm{WP}}^{\mathrm{H}}=K_{\mathrm{el}}=€ 66 / \mathrm{MWh} \quad K_{\mathrm{kon}}^{\mathrm{H}}=€ 22,5 / \mathrm{MWh} \\
\frac{K_{\mathrm{A}}}{K_{\mathrm{B}}}=\frac{K_{\mathrm{WP}}^{\mathrm{H}}}{K_{\mathrm{kon}}^{\mathrm{H}}}=\frac{€ 66 / \mathrm{MWh}}{€ 22,5 / \mathrm{MWh}} \\
\rightarrow \frac{K_{\mathrm{A}}}{K_{\mathrm{B}}}=2,9333 \\
\Rightarrow F_{\text {ges }}^{\mathrm{H}}=5,2
\end{gathered}
$$

- Ermittlung der relativen Energiekosteneinsparung im Heizbetrieb $\widehat{E}^{\mathrm{H}}$ :

$$
\begin{gathered}
C O P_{\max }^{\mathrm{H}}=6 \quad F_{\text {ges }}^{\mathrm{H}}=5,2 \\
\Rightarrow \widehat{E}^{\mathrm{H}}=0,14
\end{gathered}
$$




\section{Bestimmung der Amortisationsdauer $\Delta t_{\mathrm{A}}$}

- Ermittlung der absoluten Einsparung im Betrachtungszeitraum $E\left(\Delta t_{\mathrm{P}}\right)$ :

$$
\begin{gathered}
K_{\text {kon }}^{\mathrm{H}}=€ 22,5 / \mathrm{MWh} \\
G K_{\mathrm{kon}}^{\mathrm{H}}\left(P_{\mathrm{i}}\right)=G K_{\mathrm{kon}}^{\mathrm{H}}\left(P_{\mathrm{t}}\right)=\frac{Q_{\mathrm{N}}^{\mathrm{H}}}{\eta_{\mathrm{kon}}^{\mathrm{H}}} K_{\mathrm{kon}}^{\mathrm{H}}=\frac{11.113,43 \mathrm{MWh}}{0,85} \cdot € 22,5 / \mathrm{MWh} \\
\rightarrow G K_{\mathrm{kon}}^{\mathrm{H}}\left(P_{\mathrm{t}}\right)=€ 294.179,10 \\
E\left(\Delta t_{\mathrm{P}}\right)=G K_{\mathrm{kon}}^{\mathrm{H}}\left(P_{\mathrm{t}}\right) \widehat{E}^{\mathrm{H}}\left(P_{\mathrm{t}}\right)=€ 294.179,10 \cdot 0,14 \\
\rightarrow E\left(\Delta t_{\mathrm{P}}\right)=€ 41.185,07
\end{gathered}
$$

- Ermittlung der Amortisationsdauer $\Delta t_{\mathrm{A}}$ :

$$
\begin{gathered}
\Delta t_{\mathrm{A}}=\Delta t_{\mathrm{P}} \frac{I K_{\mathrm{WP}}}{E\left(\Delta t_{\mathrm{P}}\right)}=8760 \mathrm{~h} \cdot \frac{€ 412.313,43}{€ 41.185,07} \\
\rightarrow \Delta t_{\mathrm{A}}=10,01 \text { Jahre }
\end{gathered}
$$

Werden die graphisch ermittelbaren technischen und wirtschaftlichen Kennzahlen auf dem Rechenweg bestimmt, so ergibt sich eine Amortisationsdauer von:

$$
\Delta t_{\mathrm{A}}=7,96 \text { Jahre }
$$

Die große Abweichung ist durch Fehler bei der Ermittlung von $F_{\text {ges }}^{\mathrm{H}}$ und $\widehat{E}^{\mathrm{H}} \mathrm{zu}$ begründen. Für das Beispiel folgt:

Wenn die spezifischen Kosten der Wärmepumpe um $10 \%$ steigen und die der Vergleichstechnologie um 10 \% fallen, so erhöht sich die Amortisationsdauer im vorliegenden Fall auf ungefähr das 2-fache des Ausgangswerts.

Aus Sicht des Unternehmens ist das Resultat gerade noch zufriedenstellend. Auch bei sich für die Wärmepumpe negativ auswirkenden Veränderungen der spezifischen Kosten, liegt der Amortisationszeitraum bei maximal 10 Jahren. Noch zu ermitteln wäre, wie sich die absoluten wirtschaftlichen Kennzahlen verhalten, wenn anstelle der Luftkühlung eine Kältemaschine mit einem $C O P_{\text {real }}^{\mathrm{Kv}}$ von 5 installiert werden würde und diese 6 Monate pro Jahr im Einsatz wäre. Für die Bewertung sind die spezifischen Kosten nach Kapitel $5.4 \mathrm{zu}$ verwenden. 


\subsection{Direkte Ermittlung von $E\left(\Delta t_{\mathrm{P}}\right)$ und $\Delta t_{\mathrm{A}}$ im Heiz- und Kühlbetrieb}

Bei der Ermittlung der absoluten Einsparung im Betrachtungszeitraum $E\left(\Delta t_{\mathrm{P}}\right)$ sowie der Amortisationsdauer $\Delta t_{\mathrm{A}}$ im Heiz- und Kühlbetrieb, muss zuerst die relative Energiekosteneinsparung im Heiz- und Kühlbetrieb $\widehat{E}^{\mathrm{H}+\mathrm{K}}$ bestimmt werden. Hierbei werden nur bisher nicht gezeigte Schritte anhand von Diagrammen dargestellt.

Bestimmung der relativen wirtschaftlichen Kennzahlen im Heizund Kühlbetrieb

- Ermittlung des Gesamteinflussfaktors im Heiz- und Kühlbetrieb $F_{\text {ges }}^{\mathrm{H}+\mathrm{K}}$ :

$$
\begin{gathered}
F_{\text {ges }}^{\mathrm{H}+\mathrm{K}}=\frac{K_{\mathrm{kon}}^{\mathrm{K}}}{K_{\mathrm{kon}}^{\mathrm{H}}} \frac{\eta_{\mathrm{kon}}^{\mathrm{H}}}{\eta_{\mathrm{kon}}^{\mathrm{K}}} \\
\eta_{\mathrm{A}}=\eta_{\mathrm{kon}}^{\mathrm{K}}=1 \quad \eta_{\mathrm{B}}=\eta_{\mathrm{kon}}^{\mathrm{H}}=0,85 \\
\Rightarrow \frac{\eta_{\mathrm{B}}}{\eta_{\mathrm{A}}}=\frac{\eta_{\mathrm{kon}}^{\mathrm{H}}}{\eta_{\mathrm{kon}}^{\mathrm{K}}}=0,85 \\
K_{\mathrm{kon}}^{\mathrm{K}}=\frac{K_{\mathrm{el}}}{C O P_{\text {real }}^{\mathrm{Kv}}}=\frac{1}{5} \cdot € 60 / \mathrm{MWh} \\
\rightarrow K_{\mathrm{kon}}^{\mathrm{K}}=€ 12 / \mathrm{MWh} \\
K_{\mathrm{kon}}^{\mathrm{K}}=€ 12 / \mathrm{MWh} \\
\frac{K_{\mathrm{A}}}{K_{\mathrm{B}}}=\frac{K_{\mathrm{kon}}^{\mathrm{K}}}{K_{\mathrm{kon}}^{\mathrm{H}}}=\frac{K_{\mathrm{kon}}^{\mathrm{H}}=€ 25 / \mathrm{MWh}}{€ 25 / \mathrm{MWh}} \\
\rightarrow \frac{K_{\mathrm{A}}}{K_{\mathrm{B}}}=0,48 \\
\Rightarrow F_{\text {ges }}^{\mathrm{H}+\mathrm{K}}=0,4
\end{gathered}
$$




\section{Bestimmung des Heizkostenfaktors $\boldsymbol{X}_{\mathbf{H}}$}

Grundsätzlich kann der Heizkostenfaktor $X_{\mathrm{H}}$ oder der Kühlkostenfaktor $X_{\mathrm{K}}$ für die Ermittlung von $\widehat{E}^{\mathrm{H}+\mathrm{K}}$ herangezogen werden. Im vorliegenden Fall ist $X_{\mathrm{H}}$ aus Abbildung 5.13 bestimmt worden:

$$
\begin{gathered}
F_{\mathrm{ges}}^{\mathrm{H}+\mathrm{K}}=0,4 \quad \widehat{Q}=0,67 \\
\Rightarrow X_{\mathrm{H}}=0,77
\end{gathered}
$$

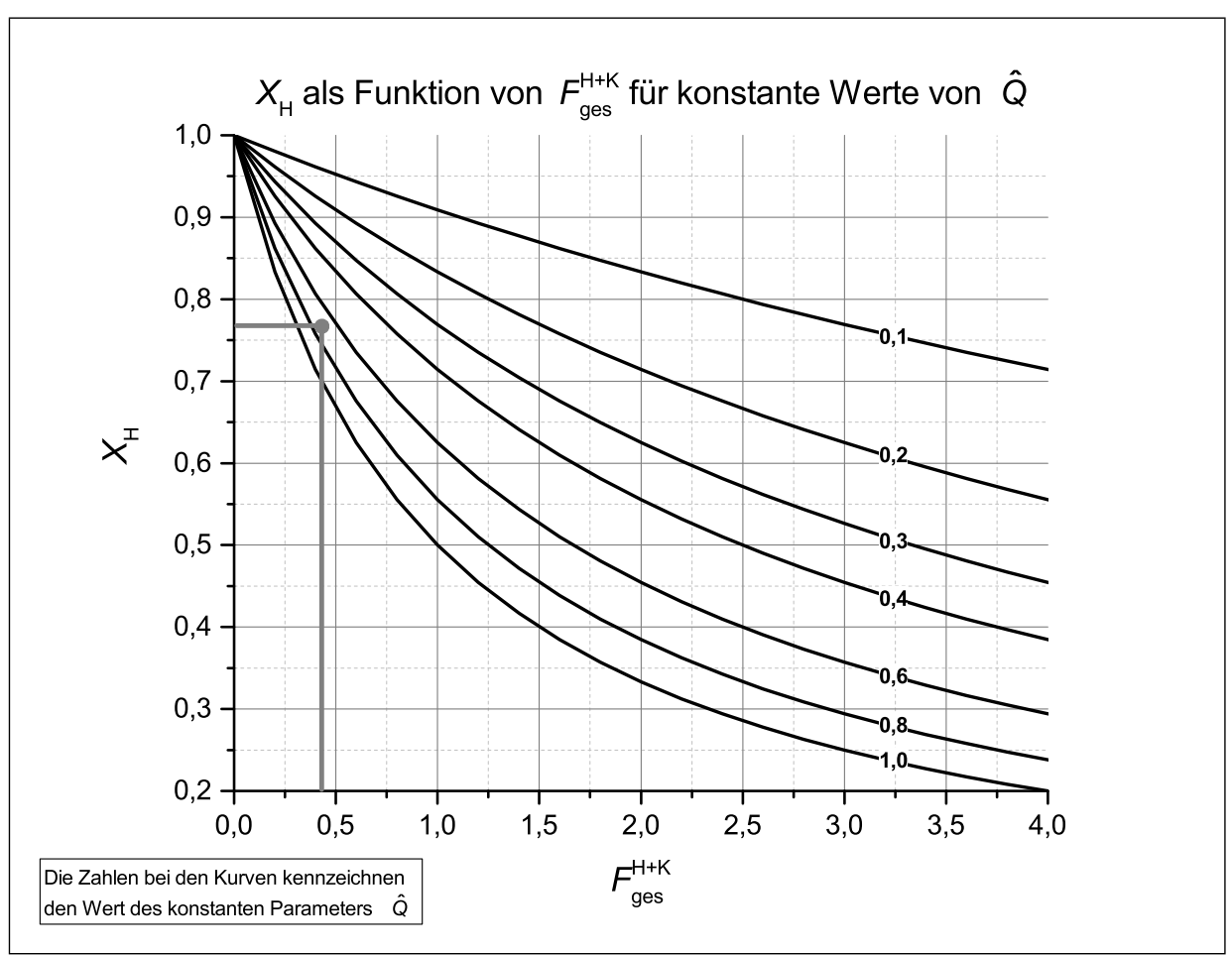

Abbildung 5.13: Fallbeispiel: Ermittlung von $X_{\mathrm{H}}$

\section{Bestimmung der relativen Energiekosteneinsparung im Heiz- und Kühlbetrieb $\widehat{\boldsymbol{E}}^{\mathbf{H}+\mathbf{K}}$}

Für die ermittelte Kombination aus Heizkostenfaktor und relativer Energiekosteneinsparung im Heizbetrieb, lässt sich aus Abbildung 5.14 die relative Energiekosteneinsparung im Heizund Kühlbetrieb ablesen:

$$
\begin{aligned}
X_{\mathrm{H}} & =0,77 \quad \widehat{E}^{\mathrm{H}}=0,31 \\
& \Rightarrow \widehat{E}^{\mathrm{H}+\mathrm{K}}=0,48
\end{aligned}
$$




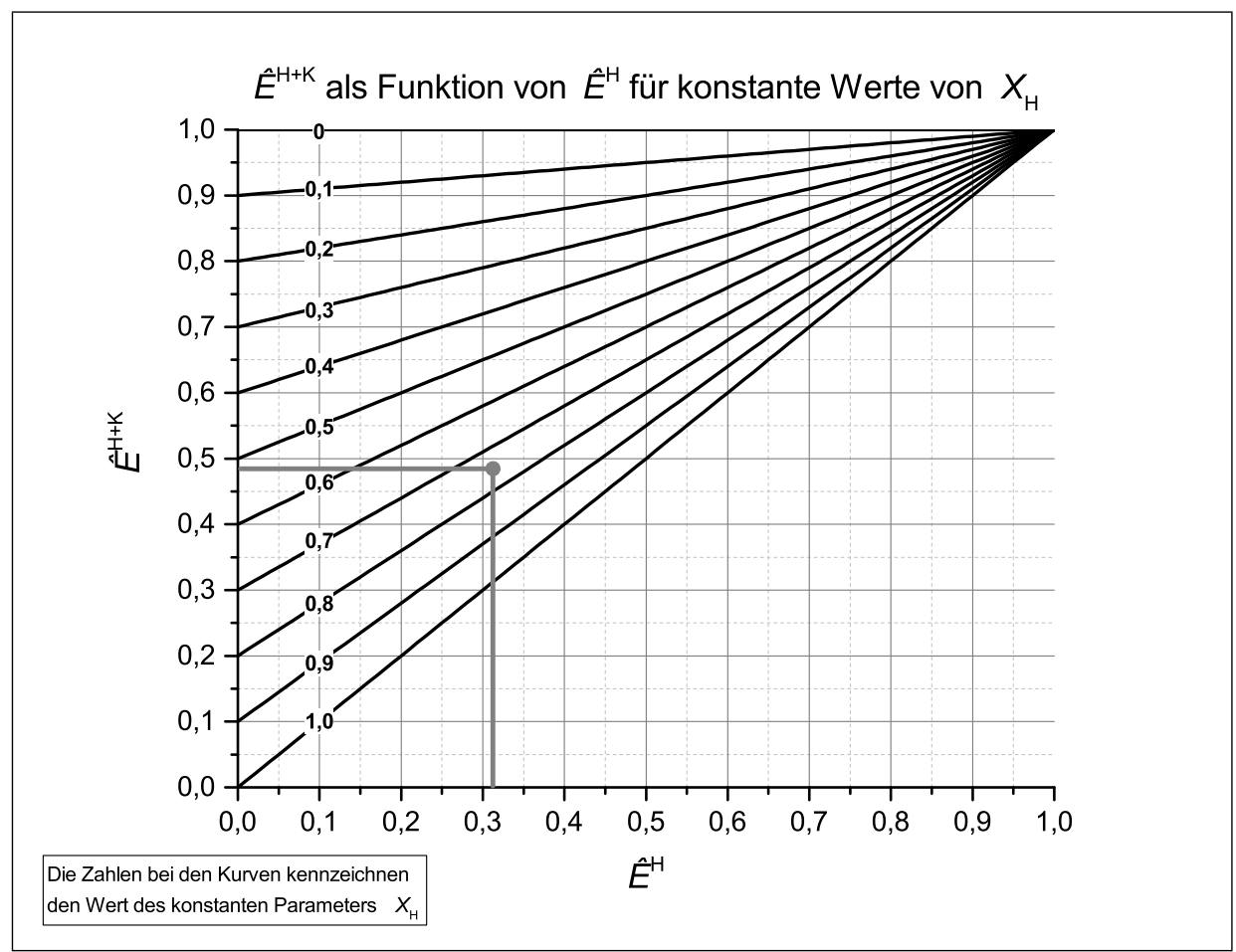

Abbildung 5.14: Fallbeispiel: Ermittlung von $\widehat{E}^{\mathrm{H}+\mathrm{K}}$

\section{Bestimmung der absoluten wirtschaftlichen Kennzahlen im Heiz- und Kühlbetrieb}

Im Gegensatz zu den vorherigen Ausführungen, befindet sich im Betrachtungszeitraum $\Delta t_{\mathrm{P}}$ mehr als ein Betriebspunkt, da das Kühlen nur für 6 Monate pro Jahr mit Kosten verbunden ist. Es gilt daher:

$$
\begin{gathered}
\Delta t_{\mathrm{P}}=\Delta t_{1}+\Delta t_{2}=8760 \mathrm{~h} \\
\Delta t_{1}=\Delta t_{2}=4.380 \mathrm{~h}
\end{gathered}
$$

Im Folgenden wird gesetzt:

- $\Delta t_{1}$ kennzeichnet den Zeitraum in dem das Kühlen mit keinen Kosten verbunden ist.

- $\Delta t_{2}$ kennzeichnet den Zeitraum in dem das Kühlen mit Kosten verbunden ist.

Die absolute Einsparung $E\left(\Delta t_{1}\right)$ beträgt die Hälfte, der in Kapitel 5.4 ermittelten absoluten Einsparung (vgl. Gleichung (5.5)):

$$
\begin{aligned}
& E\left(\Delta t_{1}\right)=\frac{1}{2} \cdot € 101.328,36 \\
& \rightarrow E\left(\Delta t_{1}\right)=€ 50.664,18
\end{aligned}
$$


Für die absolute Einsparung $E\left(\Delta t_{2}\right)$ gilt, dass sich diese aus den Gesamtkosten beim Heizen und Kühlen mit der Vergleichstechnolgie und der relativen Einsparung im Heiz- und Kühlbetrieb errechnet:

$$
\begin{gathered}
Q_{\mathrm{N}}^{\mathrm{H}}\left(P_{2}\right)=\Delta t_{2} \dot{Q}_{\mathrm{N}}^{\mathrm{H}}\left(P_{2}\right)=4.380 \mathrm{~h} \cdot 1.268,66 \mathrm{~kW} \\
\rightarrow Q_{\mathrm{N}}^{\mathrm{H}}\left(P_{2}\right)=5.556 .716 \mathrm{kWh}=5.556,72 \mathrm{MWh} \\
K_{\mathrm{kon}}^{\mathrm{H}}=€ 25 / \mathrm{MWh} \\
G K_{\mathrm{kon}}^{\mathrm{H}}\left(P_{1}\right)=G K_{\mathrm{kon}}^{\mathrm{H}}\left(P_{1}\right)=\frac{5.556,72 \mathrm{MWh}}{0,85} \cdot € 25 / \mathrm{MWh} \\
\rightarrow G K_{\mathrm{kon}}^{\mathrm{H}}\left(P_{\mathrm{t}}\right)=€ 163.432,84 \\
Q_{\mathrm{N}}^{\mathrm{K}}\left(P_{2}\right)=\Delta t_{2} \dot{Q}_{\mathrm{N}}^{\mathrm{K}}\left(P_{2}\right)=4.380 \mathrm{~h} \cdot 850 \mathrm{~kW} \\
\rightarrow Q_{\mathrm{N}}^{\mathrm{K}}\left(P_{2}\right)=3.723 .000 \mathrm{kWh}=3.723,00 \mathrm{MWh} \\
K_{\mathrm{kon}}^{\mathrm{K}}=€ 12 / \mathrm{MWh} \\
G\left(\Delta t_{2}\right)=G K_{\mathrm{kon}}^{\mathrm{H}+\mathrm{K}}\left(P_{2}\right) \widehat{E}^{\mathrm{H}+\mathrm{K}}\left(P_{2}\right)=(€ 163.432,84+€ 44.676,00) \cdot 0,48 \\
\rightarrow E\left(\Delta t_{2}\right)=€ 99.892,24 \\
G K_{\mathrm{kon}}^{\mathrm{H}}\left(P_{2}\right)=G K_{\mathrm{kon}}^{\mathrm{K}}\left(P_{2}\right)=\frac{3.723,00 \mathrm{MWh}}{1} \cdot € 12 / \mathrm{MWh} \\
\left.\rightarrow P_{2}\right)=€ 44.676,00
\end{gathered}
$$

Daraus folgt für die absolute Einsparung im Zeitraum $\Delta t_{\mathrm{P}}$ :

$$
\begin{gathered}
E\left(\Delta t_{1}\right)=€ 50.664,18 \quad E\left(\Delta t_{2}\right)=€ 99.892,24 \\
E\left(\Delta t_{\mathrm{P}}\right)=E\left(\Delta t_{1}\right)+E\left(\Delta t_{2}\right)=€ 50.664,18+€ 99.892,24 \\
\rightarrow E\left(\Delta t_{\mathrm{P}}\right)=€ 150.556,42
\end{gathered}
$$

woraus sich, unter Verwendung des bereits bekannten Formelwerks, die Amortisationsdauer $\Delta t_{\mathrm{A}}$ errechnen lässt:

$$
\begin{gathered}
\Delta t_{\mathrm{A}}=\Delta t_{\mathrm{P}} \frac{I K_{\mathrm{WP}}}{E\left(\Delta t_{\mathrm{P}}\right)}=8760 \mathrm{~h} \cdot \frac{€ 412.313,43}{€ 150.556,42} \\
\rightarrow \Delta t_{\mathrm{A}}=2,74 \text { Jahre }
\end{gathered}
$$


Werden die graphisch ermittelbaren technischen und wirtschaftlichen Kennzahlen auf dem Rechenweg bestimmt, so ergibt sich eine Amortisationsdauer von:

$$
\Delta t_{\mathrm{A}}=2,73 \text { Jahre }
$$

Für das Beispiel folgt:

Die Amortisationsdauer im Heiz- und Kühlbetrieb liegt, je nachdem ob der graphisch oder rechnerisch ermittelte Wert als Bezugspunkt herangezogen wird, ungefähr ein Drittel unter der Amortisationsdauer im reinen Heizbetrieb.

\subsection{Fazit}

Zusammenfassend lässt sich feststellen, dass das entwickelte Bewertungskonzept grundsätzlich in der Lage ist, die gegebenen Aufgabenstellungen mit hinreichender Genauigkeit zu bearbeiten. Einzig im Bereich der graphischen Ermittlung der relativen Energiekosteneinsparung im Heizbetrieb $\widehat{E}^{\mathrm{H}}$ ist, auf Grund der hohen Änderungsraten von $\widehat{E}^{\mathrm{H}}$, ein Ablesen häufig mit großen Fehlern verbunden. Dieser Punkt wird separat in Kapitel 6.2.2 adressiert. 


\section{Diskussion und Ausblick}

Das grundsätzliche Ziel der vorliegenden Arbeit, die Entwicklung eines Schemas zur Bewertung der Leistungsfähigkeit von Wärmepumpen im Heiz- und Kühlbetrieb, kann als erfüllt angesehen werden. Nachdem es sich bei dem vorgestellten Bewertungsansatz jedoch um ein neuartiges Konzept handelt, kann zum jetzigen Zeitpunkt nicht festgestellt werden, welche der Ansätze sich als funktional erweisen werden, welche einer Nachbearbeitung bedürfen und welche ggf. gar nicht zur Anwendung kommen werden. In diesem Kapitel werden, aus Sicht des Autors, Beobachtungen diskutiert und ein Ausblick gegeben. Falls möglich und sinnvoll, so werden sowohl Ausblick als auch Diskussion jeweils im selben Unterkapitel zusammengefasst.

Nachdem das gesamte Dokument nach technischen, relativen wirtschaftlichen und absoluten wirtschaftlichen Kennzahlen gegliedert ist, wird diese Unterteilung auch in diesem Kapitel beibehalten.

\subsection{Technische Kennzahlen}

Der Bereich der technischen Kennzahlen wird von der Bestimmung des maximalen COPs im Heizbetrieb dominiert. Für die anderen technischen Kennzahlen sind keine neuen Zusammenhänge abgeleitet worden bzw. haben diese, wie z.B. bei $C O P_{\text {real }}^{\mathrm{H}+\mathrm{K}}$, keine Verwendung gefunden.

\section{Maximaler COP im Heizbetrieb $C O P_{\max }^{\mathrm{H}}$}

Zur Bestimmung von $C O P_{\max }^{\mathrm{H}}$ sind zwei Ansätze vorgestellt worden:

Ansatz 1: Bestimmung von $C O P_{\max }^{\mathrm{H}}$ unter Verwendung von $C O P_{0}^{\mathrm{H}}, \widehat{\Delta T}$ und $X_{\mathrm{T}}$.

Ansatz 2: Bestimmung von $C O P_{\max }^{\mathrm{H}}$ auf Basis von $\overline{C O P_{\max }^{\mathrm{H}}}$. 


\section{Ansatz 1}

Der erste Ansatz ist verhältnismäßig kompliziert und es muss diskutiert werden, ob er die Anforderungen, die an das gesamte Schema gestellt werden, in allen Bereichen vollständig erfüllt. In diesem Zusammenhang ist anzumerken:

- Bei der direkten Anwendung auf eine eingeschränkte Anzahl an Quellen und Senken ist dieser Ansatz als tauglich einzustufen, auch wenn grundsätzlich hinterfragt werden muss, ob es nicht einen einfacheren Weg der Bestimmung, ggf. unter Einführung anderer Größen, gäbe.

- Der Ansatz ist für die Beantwortung der inversen Fragestellung nur sehr eingeschränkt geeignet. Es ist zwar möglich, setzt aber voraus, dass sich eingehend mit diesem Ansatz beschäftigt wurde. Diese Voraussetzung steht jedoch dem Anspruch, dass das zu entwickelnde Konzept leicht verständlich und einfach in der Anwendung sein soll, diametral gegenüber. Auch ist es schwer zumutbar, wenn manche Ablesevorgänge beim inversen Einsatz iterativ durchgeführt werden müssen.

- Die Diagramme für $C O P_{0}^{\mathrm{H}}$ bei $C O P_{0}^{\mathrm{H}}=$ konstant erfüllen nicht ihren Zweck und es ist zu überprüfen, ob diese nicht entfernt werden sollten.

- Die Diagramme zur Bestimmung von $X_{\mathrm{T}}$ sind schlecht ablesbar. In diesem Zusammenhang wäre festzustellen, welcher Wertebereich für $\widehat{\Delta T}$ in der industriellen Anwendung tatsächlich wahrscheinlich ist. Im Anschluss müssten die Diagramme für $X_{\mathrm{T}}$ dahingehend angepasst werden. Dies gilt ebenfalls für $C O P_{0}^{\mathrm{H}}$, wobei der Einfluss von $C O P_{0}^{\mathrm{H}}$ auf $X_{\mathrm{T}}$ im Bereich von großen Werten für $C O P_{0}^{\mathrm{H}}$ relativ gering ist. Eventuell könnte es daher sinnvoll sein, die Diagramme für $X_{\mathrm{T}}$ nur bis zu einem bestimmten Wert für $C O P_{0}^{\mathrm{H}}$ darzustellen, wenn der daraus resultierende Fehler in der Anwendung hinreichend klein ist. 


\section{Ansatz 2}

Der zweite Ansatz ist in der Anwendung wesentlich schneller als der erste, jedoch nur für die inverse Problemstellung geeignet. In diesem Kontext ist zu diskutieren:

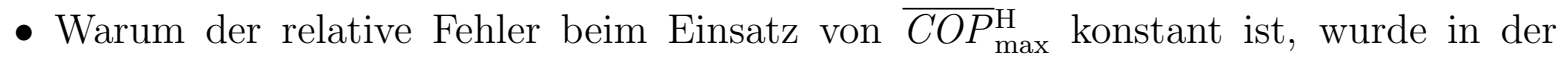
vorliegende Arbeit nicht erläutert. Eine mathematisch einwandfreie Herleitung ist an dieser Stelle zu empfehlen.

- Die Ermittlung von $\overline{C O P_{\max }^{\mathrm{H}}}$ für den Fall, dass die Kombination aus $\vartheta_{\mathrm{S}}$ und $\Delta T_{\mathrm{WT}}$ nicht anhand von Abbildung 3.14 und Tabelle 3.1 ermittelbar ist, ist in der vorliegenden Arbeit nicht beschrieben worden. Dies gilt ebenfalls für die Fragestellung, welche Aussage für Punkte bzw. Kurven zwischen den in Abbildung 3.14 dargestellten Kurven getätigt werden kann. An diesen Stellen sei eine Nachbearbeitung dringend empfohlen.

- Für die Anwendung wäre eine graphische Darstellung von $\overline{C O P_{\max }^{\mathrm{H}}}=$ konstant äußerst hilfreich. Es ist zu überprüfen, ob es möglich ist, derartige Diagramme für $\overline{C O P}_{\max }^{\mathrm{H}}$ zu erzeugen.

\subsection{Wirtschaftliche Kennzahlen}

Im Bereich der wirtschaftliche Kennzahlen sind eine Vielzahl an Größen zur Bestimmung der relativen wirtschaftliche Kennzahlen eingeführt und visualisiert worden. Die absoluten technischen Kennzahlen dienen in der vorliegenden Arbeit primär als Ergänzung, um die Szenarien des Fallbeispiels vollständig darstellen zu können.

\subsubsection{Relative wirtschaftliche Kennzahlen}

Die Ableitung der relativen wirtschaftliche Kennzahlen war, im Gegensatz zu den technischen Kennzahlen, ohne die Einführung von schwer zu interpretierenden Größen möglich. Durch die Einführung des Gesamteinflussfaktors wurde weiteres gewährleistet, dass die Anzahl, der für die Bewertung benötigen Diagramme, verhältnismäßig klein ist. Im Bereich der relativen wirtschaftlichen Kennzahlen ist anzumerken:

- Die Diagramme zur Ermittlung von $\widehat{E}^{\mathrm{H}}$ müssen sehr sorgfältig abgelesen werden. Speziell im Bereich von niedrigen Werten für $F_{\text {ges }}^{\mathrm{H}}$ und $C O P_{\max }^{\mathrm{H}}$ kann es häufig zu schweren Ablesefehler kommen. Es ist daher zu überprüfen, ob eine andere, einfacher abzulesende Darstellung von $\widehat{E}^{\mathrm{H}}$ möglich ist. 
- Diagramme für konstante Werte von $\widehat{E}^{\mathrm{H}}$ könnten sich in der Anwendung als nützlich erweisen, da sie die Ermittlung jener Kombinationen aus $F_{\text {ges }}^{\mathrm{H}}$ und $C O P_{\max }^{\mathrm{H}}$, die für bestimmte konstante Werte von $\widehat{E}^{\mathrm{H}}$ benötigt würden, ermöglichen könnten. Im Rahmen einer Nachbearbeitung des Bewertungskonzepts, könnte diese Idee einer kritischen Überprüfung unterzogen werden.

- Das Konzept der Wirtschaftlichkeitsgrenze im Heizbetrieb ist in der Implementierung seiner möglichen Aussagekraft nicht gerecht geworden. Das Zusammenspiel zwischen $F_{\text {ges }}^{\mathrm{H}}, C O P_{\max }^{\mathrm{H}}$ und $\widehat{E}^{\mathrm{H}}$ könnte in weiteren Arbeiten stärker herausgearbeitet werden. Nachdem der Konversionswirkungsgrad in den meisten Fällen konstant sein wird, bietet es sich in diesem Zusammenhang auch an, den Einfluss des Verhältnisses der spezifischen Kosten auf die Wirtschaftlichkeitsgrenze und $\widehat{E}^{\mathrm{H}}$ zu diskutieren.

- Die Einführung von $F_{\text {ges }}^{\mathrm{H}+\mathrm{K}}$ als Quotient aus $F_{\text {ges }}^{\mathrm{H}}$ und $F_{\text {ges }}^{\mathrm{K}}$ hat sich für das Schema als zweckmäßig erwiesen, ist jedoch vor dem Hintergrund der Definition von $F_{\mathrm{B}}^{\mathrm{A}}$ kritisch zu hinterfragen. Es ist zu überprüfen, ob $F_{\text {ges }}^{\mathrm{H}+\mathrm{K}}$ nicht vielmehr als Quotient aus den Gesamtkosten der Vergleichstechnologie im Heizbetrieb und den Gesamtkosten der Vergleichstechnologie im Kühlbetrieb, unter der Nebenbedingung $Q_{\mathrm{N}}^{\mathrm{H}}=Q_{\mathrm{N}}^{\mathrm{K}}$, vorgestellt werden sollte und ob hierfür ggf. $F_{\mathrm{B}}^{\mathrm{A}}$ anders definiert werden müsste oder ob es ausreicht, dies als Sonderfall in der die Technologie A der Technologie $\mathrm{B}$ entspricht, zu interpretieren.

- Die Fallunterscheidung im Bezug auf den Gesamteinflussfaktor im Kühlbetrieb ist nicht ideal. Eventuell ist es möglich einen Ansatz zu finden, der ohne die Annahme $\eta_{\mathrm{kon}}^{\mathrm{K}}=1$ verwendbar ist. Denkbar wäre z.B. die Einführung eines Gütegrads für die Kältemaschine der Vergleichstechnologie. In diesem Fall könnten etwaige Konversionsverluste darin zusammengefasst werden. Dies würde die Ableitung eines Zusammenhangs zwischen $\eta_{\mathrm{kon}}^{\mathrm{K}}$ und dem Gütegrads der Kältemaschine der Vergleichstechnologie ermöglichen. 


\subsubsection{Absolute wirtschaftliche Kennzahlen}

Die absoluten wirtschaftlichen Kennzahlen werden in der vorliegenden Arbeit nur eingeführt, um ein vollständiges Bild im Anwendungskapitel geben zu können. Sollte sich das in der vorliegenden Arbeit entwickelte Bewertungskonzept als sinnvoll erweisen, bietet es sich an zu überprüfen, ob für die absoluten wirtschaftlichen Kennzahlen ebenfalls Ansätze gefunden werden können, die eine diagrammbasierte Ermittlung der absoluten wirtschaftlichen Kennzahlen ermöglichen.

\subsection{Allgemeine Punkte}

Neben den Fragestellungen, die spezifischen Kapiteln zugeordnet sind, gibt es noch eine Vielzahl an Punkten, die angemerkt werden sollten:

- Die Ermittlung von $\vartheta_{\mathrm{Q}}$ ist, mit Ausnahme von Wärmequellen die über latente Energieanteile verfügen und nicht unterkühlt werden, nicht für alle Medien trivial. An dieser Stelle könnte es daher zweckmäßig sein, Hilfestellungen in Form von Diagrammen oder Formeln zur Abschätzung der Temperatur der Wärmequelle nach dem Verdampfer des Wärmepumpenkreislaufs anzubieten.

- In der vorliegenden Arbeit werden nur Kaltdampf-Kompressionswärmepumpen betrachte, deren Arbeitsmedium Zustandsänderungen zwischen der gasförmigen und der flüssigen Phase vollzieht. Die Anwendung des Bewertungskonzepts auf Wärmepumpen deren Arbeitsmedium sich immer im gasförmigen Zustand befindet (vgl. [1]), ist im Status Quo nicht möglich. Eine Erweiterung in diese Richtung könnte angedacht werden.

- Der Rahmen der vorliegenden Arbeit hat es nicht erlaubt, die Ansätze zur Bewertung der Leistungsfähigkeit anhand von anderen Beispielen zu überprüfen und zu dokumentieren. Sollte sich das Bewertungskonzept als sinnvoll herausstellen, so wäre es aus der Sicht des potentiellen Anwenders hilfreich, zusätzliche Beispiele und ein Fließschema des Ablaufs der Bewertung zu ergänzen.

- Die Wärmepumpe im industriellen Umfeld ist Thema mehrerer nationaler und internationaler Forschungsprojekte. Einige dieser Projekte beschäftigen sich mit Fragestellungen rund um das Thema der Hochtemperaturwärmepumpe. Die Anwendung des in dieser Arbeit entwickelten Bewertungskonzept auf derartig gelagerte Fragestellungen wäre eventuell in der Lage, interessante Resultate, im Bezug auf die quellseitigen Anforderungen einer Hochtemperaturpumpe, hervorzubringen. 
- Wärmepumpen können auf verschiedene Arten in Prozesse eingebunden werden. Nicht immer wird daher nur der direkte Vergleich zwischen der Wärmepumpe und einer anderen Technologie gefordert sein. Häufig werden sich aus selbst einfach anmutenden Ausgangssituationen sehr komplexe Verschaltungskonzepte ergeben. Das hier entwickelte Bewertungskonzept ist bereits auf die Kombination von Geothermie und einer Wärmepumpe angewendet worden, die Resultate waren jedoch nicht verallgemeinerbar. Es bietet sich daher an, zu untersuchen, welche Werkzeuge bzw. Hilfestellungen benötigt würden, um das in dieser Arbeit entwickelte Schema zur Bewertung der Leistungsfähigkeit einer Wärmepumpe auch auf komplexere Szenarien der Verschaltung anwenden zu können. Hierbei sollten sowohl vergleichende als auch auf Zahlenwerten basierende Szenarien Berücksichtigung finden.

- Bei der Ableitung der Kennzahlen ist in der vorliegenden Arbeit häufig der Ansatz gewählt worden, möglichst viele Parameterkonstellationen zuzulassen. Es muss jedoch die Frage gestellt werden, ob ein schnelles und hinreichend genaues Bewertungskonzept nicht auch dadurch gefunden werden kann, in dem z.B. $\Delta T_{\mathrm{WT}}$ und $\eta_{\mathrm{WP}}$, mit charakteristischen Werten versehen, als konstant angenommen werden. Dadurch ergeben sich völlig andere Möglichkeiten der Darstellung, die es in nachgelagerten Tätigkeiten zu erarbeiten und zu bewerten gilt.

- Die wesentliche Größe des hier entwickelten Bewertungskonzepts ist die relative Energiekosteneinsparung. Es ist jedoch in der Zukunft nicht auszuschließen, dass ökologische Aspekte eine stärkere Gewichtung bekommen könnten. Eine Erweiterung in diese Richtung könnte sich daher als sinnvoll erweisen.

- Es gibt eine Reihe von Werkzeugen, die die Auswahl möglicher Integrationskonzepte einer Wärmepumpe erleichtern. Ein Beispiel hierfür ist die Pinchanalyse. Es ist zu überprüfen, ob aus der Pinchanalyse eventuell wertvolle Ansätze zur Reduktion der tatsächlich zu berücksichtigen Quellen und Senken ableitbar sind. Dies könnte den Bearbeitungsaufwand des gesamten Bewertungskonzepts eventuell schon zu Beginn reduzieren. 


\section{Literaturverzeichnis}

[1] B. Adler und R. Mauthner. Rotation Heat Pump (RHP). In IEA Heat Pump Conference 2017, 2017.

[2] H. D. Baehr und S. Kabelac. Thermodynamik Grundlagen und technische Anwendungen. Springer Verlag, Berlin, 15. Auflage, 2012.

[3] H. Herwig und C.H. Kautz. Technische Thermodynamik. Pearson Verlag, München, 2007.

[4] W. Eder und F. Moser. Die Wärmepumpe in der Verfahrenstechnik. Springer Verlag, Wien, 1979. 


\section{A. Diagramme und Tabellen}

Im Anhang befinden sich alle für die Bewertung benötigten Diagramme und Tabellen. 


\section{Technische Kennzahlen}

- Abbildung A.1

$\widehat{\Delta T}$ als Funktion von $\Delta T_{\mathrm{SQ}}$ für konstante Werte von $\Delta T_{\mathrm{WT}}$ im Intervall $0{ }^{\circ} \mathrm{C} \leq$ $\Delta T_{\mathrm{SQ}} \leq 25^{\circ} \mathrm{C}$

- Abbildung A.2

$\widehat{\Delta T}$ als Funktion von $\Delta T_{\mathrm{SQ}}$ für konstante Werte von $\Delta T_{\mathrm{WT}}$ im Intervall $25^{\circ} \mathrm{C} \leq$ $\Delta T_{\mathrm{SQ}} \leq 100^{\circ} \mathrm{C}$

- Abbildung A.3 $C O P_{0}^{\mathrm{H}}$ als Funktion von $\vartheta_{\mathrm{S}}$ für konstante Werte von $\vartheta_{\mathrm{Q}}$

- Abbildung A.4 $C O P_{0}^{\mathrm{H}}$ als Funktion von $\vartheta_{\mathrm{Q}}$ für konstante Werte von $\vartheta_{\mathrm{S}}$

- Abbildung A.5

$\vartheta_{\mathrm{S}}$ als Funktion von $\vartheta_{\mathrm{Q}}$ im Intervall $0{ }^{\circ} \mathrm{C} \leq \vartheta_{\mathrm{S}} \leq 200^{\circ} \mathrm{C}$ für konstante Werte von $\mathrm{COP}_{0}^{\mathrm{H}}$

- Abbildung A.6

$X_{\mathrm{T}}$ als Funktion von $C O P_{0}^{\mathrm{H}}$ im Intervall $2 \leq C O P_{0}^{\mathrm{H}} \leq 26$ für konstante Werte von $\widehat{\Delta T}$

- Abbildung A.7

$C O P_{\max }^{\mathrm{H}}$ als Funktion von $C O P_{0}^{\mathrm{H}}$ im Intervall $2 \leq C O P_{0}^{\mathrm{H}} \leq 26$ für konstante Werte von $X_{\mathrm{T}}$

- Abbildung A.8

$C O P_{\max }^{\mathrm{H}}$ als Funktion von $C O P_{0}^{\mathrm{H}}$ im Intervall $2 \leq C O P_{0}^{\mathrm{H}} \leq 10$ für konstante Werte von $X_{\mathrm{T}}$

- Abbildung A.9

$\overline{C O P}_{\max }^{\mathrm{H}}$ als Funktion von $\vartheta_{\mathrm{Q}}$ für konstante Werte von $\vartheta_{\mathrm{S}}^{1}$

- Tabelle A.1

Ablesehilfe für Abbildung A.9

- Abbildung A.10 $C O P_{\text {real }}^{\mathrm{H}}$ als Funktion von $C O P_{\max }^{\mathrm{H}}$ für konstante Werte von $\eta_{\mathrm{WP}}$

- Abbildung A.11 $C O P_{\text {real }}^{\mathrm{H}}$ als Funktion von $C O P_{\max }^{\mathrm{H}}$ im Intervall $0 \leq C O P_{\max }^{\mathrm{H}} \leq 4$ für konstante Werte von $\eta_{\mathrm{WP}}$

- Abbildung A.12

$\widehat{Q}$ als Funktion von $C O P_{\text {real }}^{\mathrm{H}}$ 


\section{Wirtschaftliche Kennzahlen}

- Abbildung A.13

Quotient aus $\eta_{\mathrm{B}}$ und $\eta_{\mathrm{A}}$ im Intervall $0,2 \leq \eta_{\mathrm{A}} \leq 1$ für konstante Werte von $\eta_{\mathrm{B}}$

- Abbildung A.14

Quotient aus $\eta_{\mathrm{B}}$ und $\eta_{\mathrm{A}}$ im Intervall $0,05 \leq \eta_{\mathrm{A}} \leq 0,2$ für konstante Werte von $\eta_{\mathrm{B}}$

- Abbildung A.15

$F_{\mathrm{B}}^{\mathrm{A}}$ als Funktion von $K_{\mathrm{A}}$ zu $K_{\mathrm{B}}$ im Intervall $0 \leq F_{\mathrm{B}}^{\mathrm{A}} \leq 20$ für konstante Werte von $\eta_{\mathrm{B}} / \eta_{\mathrm{A}}$

- Abbildung A.16

$F_{\mathrm{B}}^{\mathrm{A}}$ als Funktion von $K_{\mathrm{A}}$ zu $K_{\mathrm{B}}$ im Intervall $0 \leq K_{\mathrm{A}} / K_{\mathrm{B}} \leq 2$ und $0 \leq F_{\mathrm{B}}^{\mathrm{A}} \leq 4$ für konstante Werte von $\eta_{\mathrm{B}} / \eta_{\mathrm{A}}$

- Abbildung A.17

$\widehat{E}^{\mathrm{H}}$ als Funktion von $C O P_{\max }^{\mathrm{H}}$ für konstante Werte von $F_{\text {ges }}^{\mathrm{H}}$

- Abbildung A.18

$X_{\mathrm{H}}$ als Funktion von $F_{\text {ges }}^{\mathrm{H}+\mathrm{K}}$ für konstante Werte von $\widehat{Q}$

- Abbildung A.19

$X_{\mathrm{H}}$ als Funktion von $F_{\text {ges }}^{\mathrm{H}+\mathrm{K}}$ im Intervall $0 \leq F_{\text {ges }}^{\mathrm{H}+\mathrm{K}} \leq 4$ für konstante Werte von $\widehat{Q}$

- Abbildung A.20

$X_{\mathrm{K}}$ als Funktion von $F_{\text {ges }}^{\mathrm{H}+\mathrm{K}}$ für konstante Werte von $\widehat{Q}$

- Abbildung A.21

$X_{\mathrm{K}}$ als Funktion von $F_{\text {ges }}^{\mathrm{H}+\mathrm{K}}$ im Intervall $0 \leq F_{\text {ges }}^{\mathrm{H}+\mathrm{K}} \leq 4$ für konstante Werte von $\widehat{Q}$

- Abbildung A.22

$\widehat{E}^{\mathrm{H}+\mathrm{K}}$ als Funktion von $\widehat{E}^{\mathrm{H}}$ für konstante Werte von $X_{\mathrm{H}}$

- Abbildung A.23

$\widehat{E}^{\mathrm{H}+\mathrm{K}}$ als Funktion von $\widehat{E}^{\mathrm{H}}$ für konstante Werte von $X_{\mathrm{K}}$ 


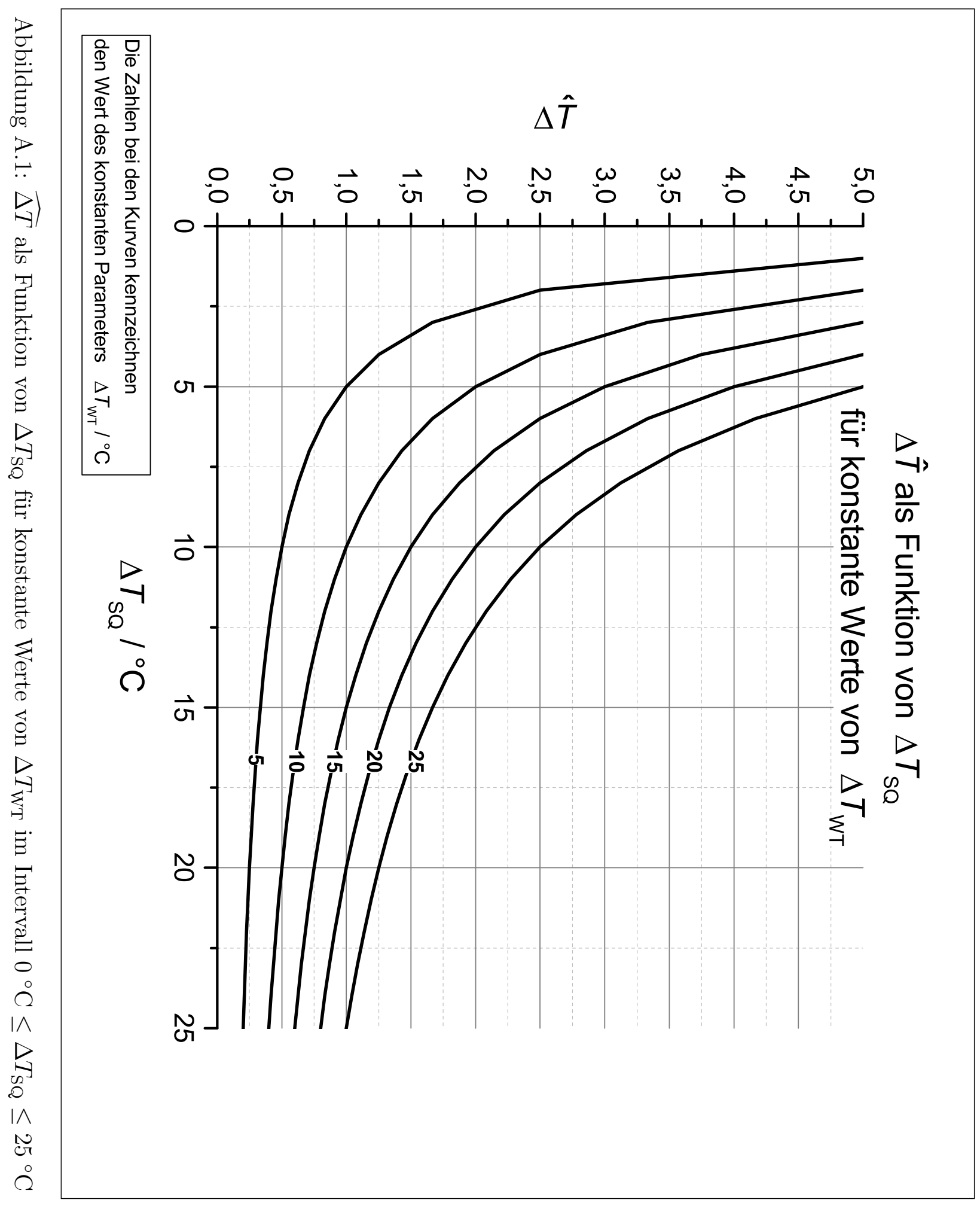




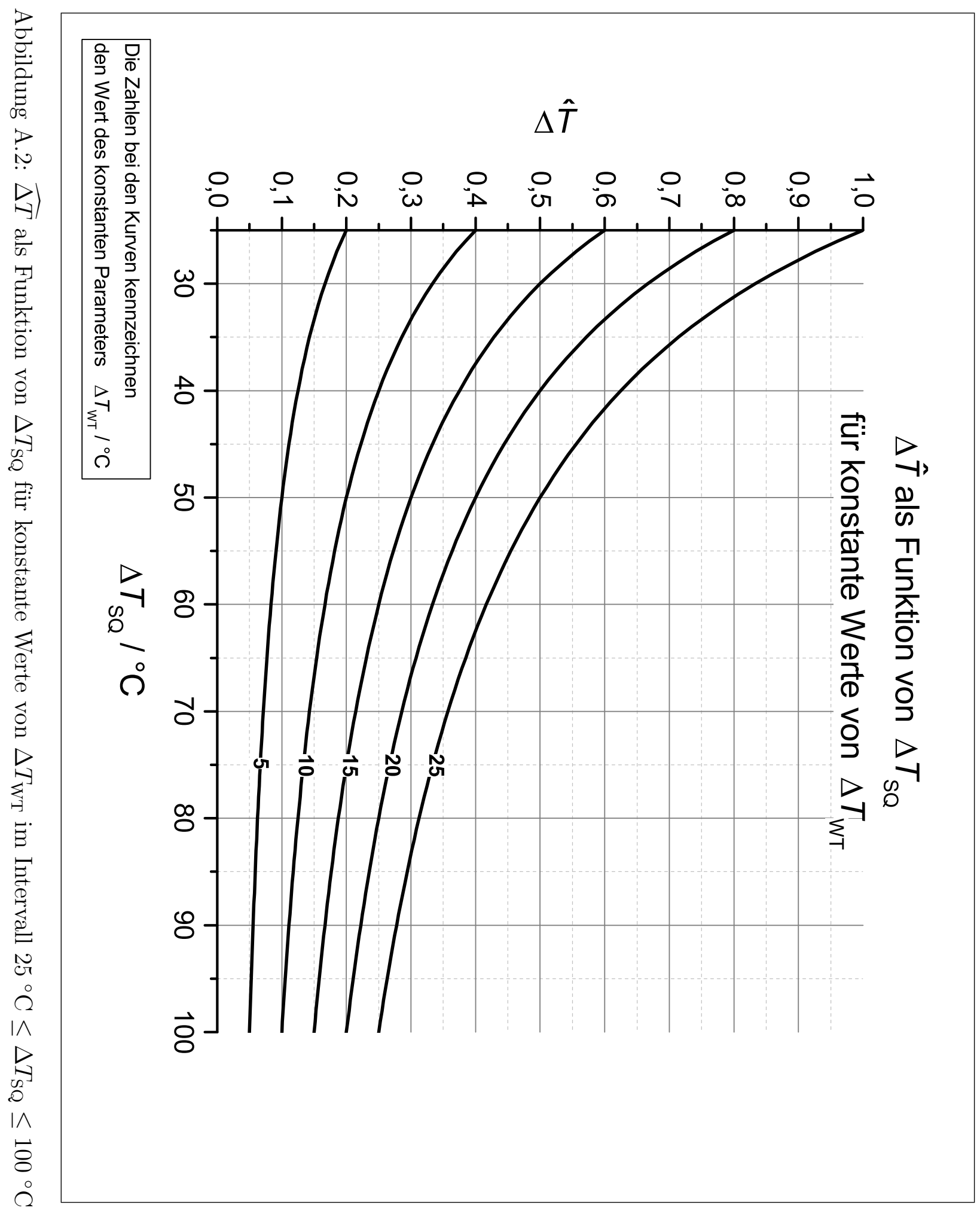




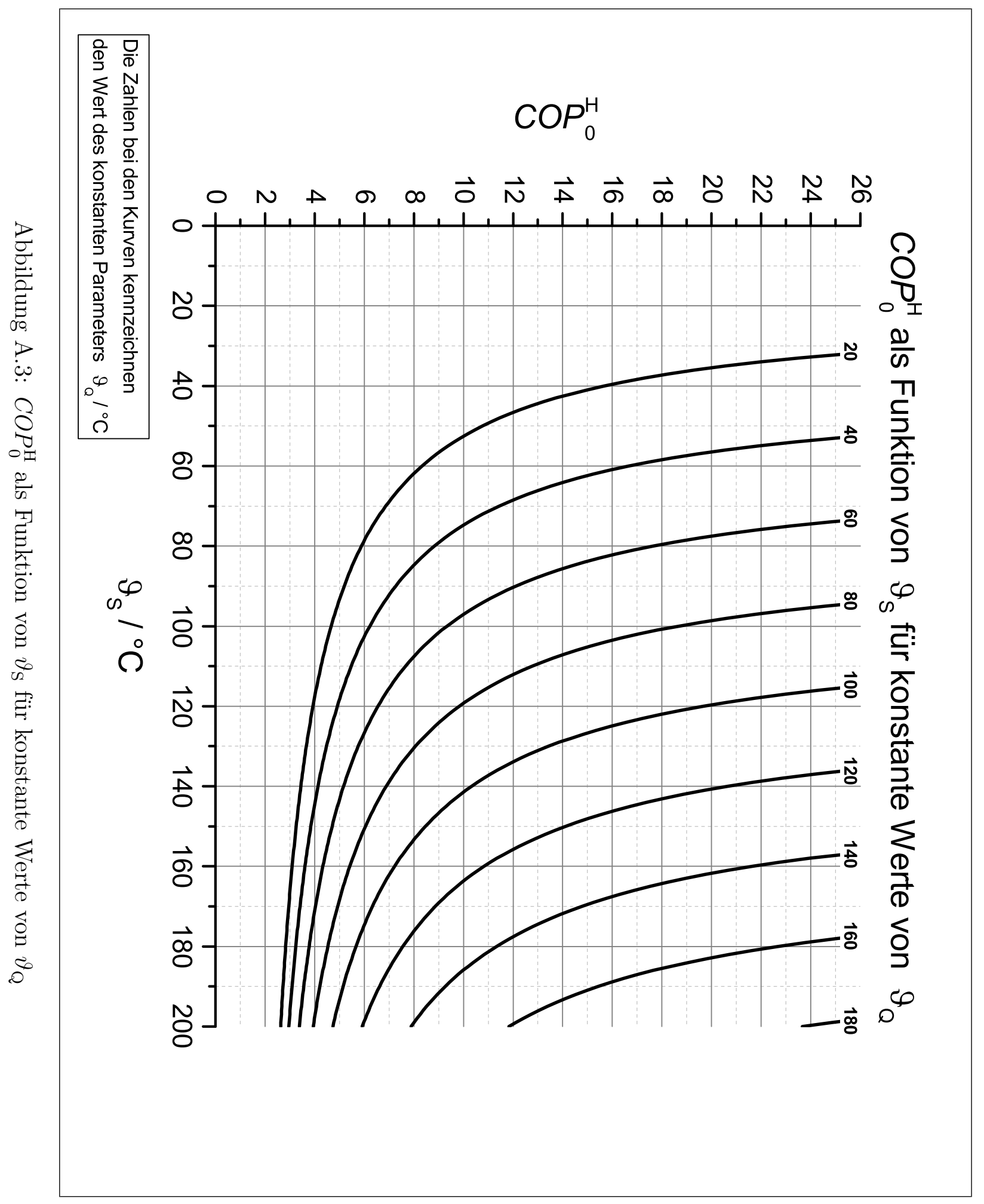




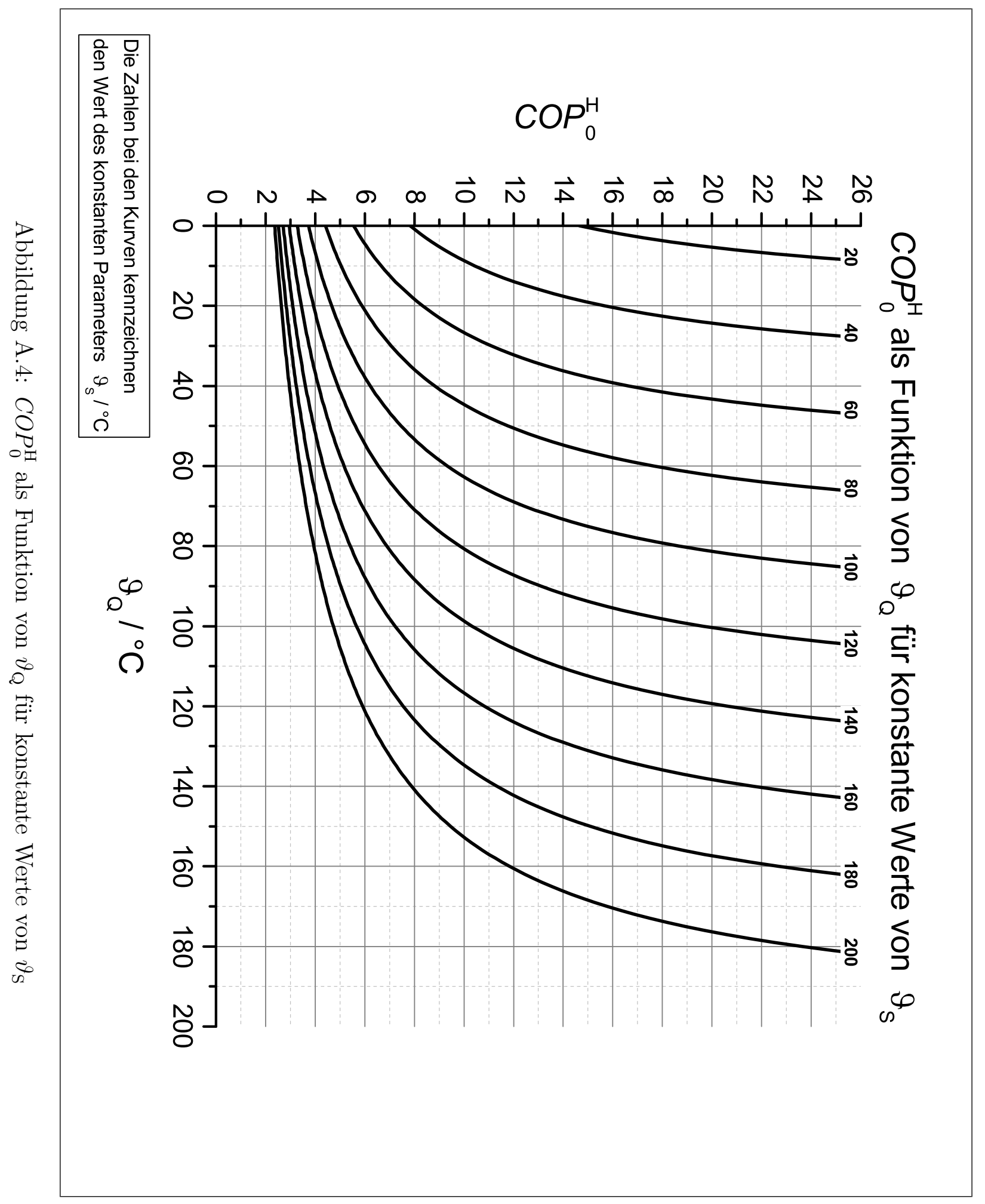




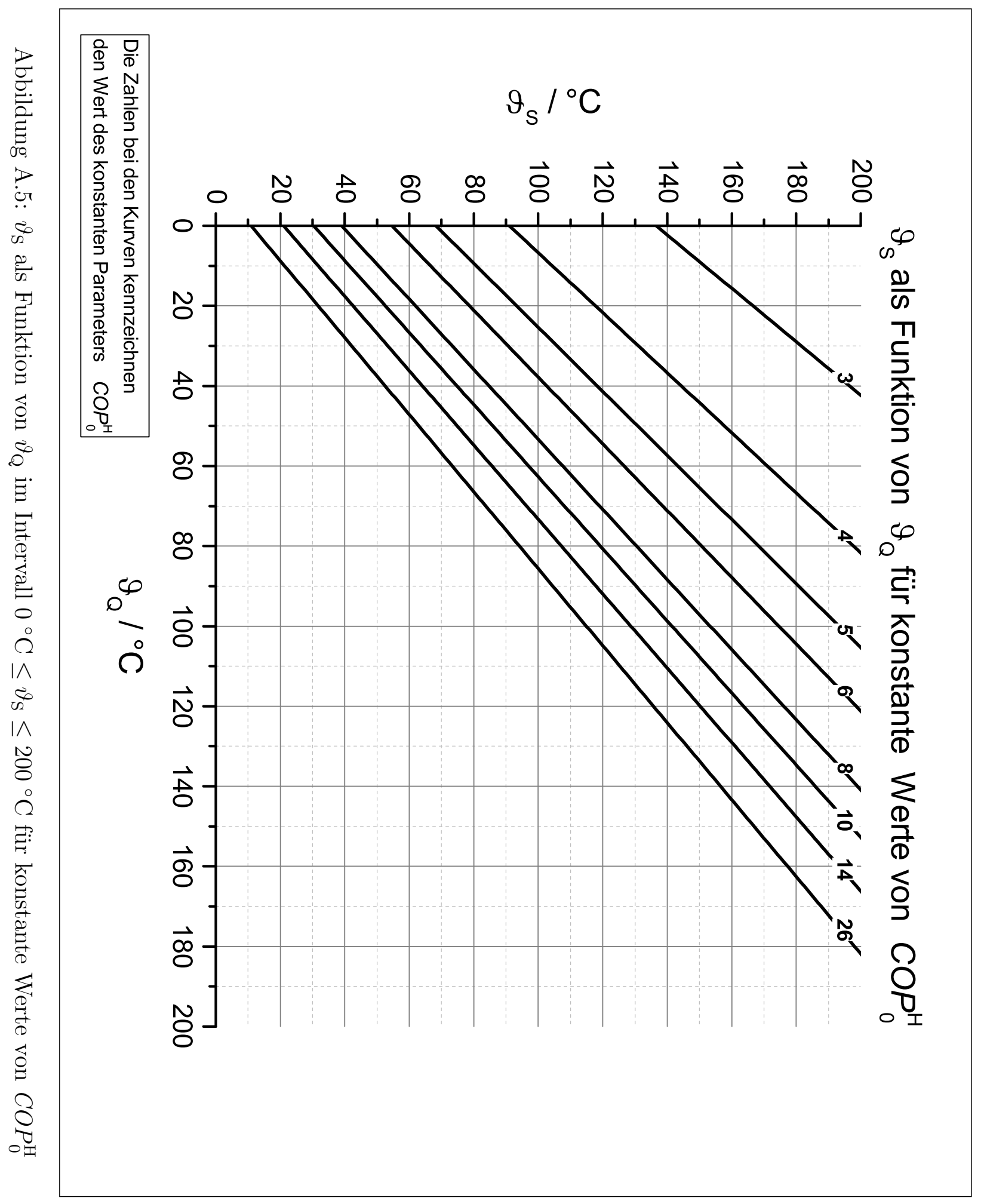




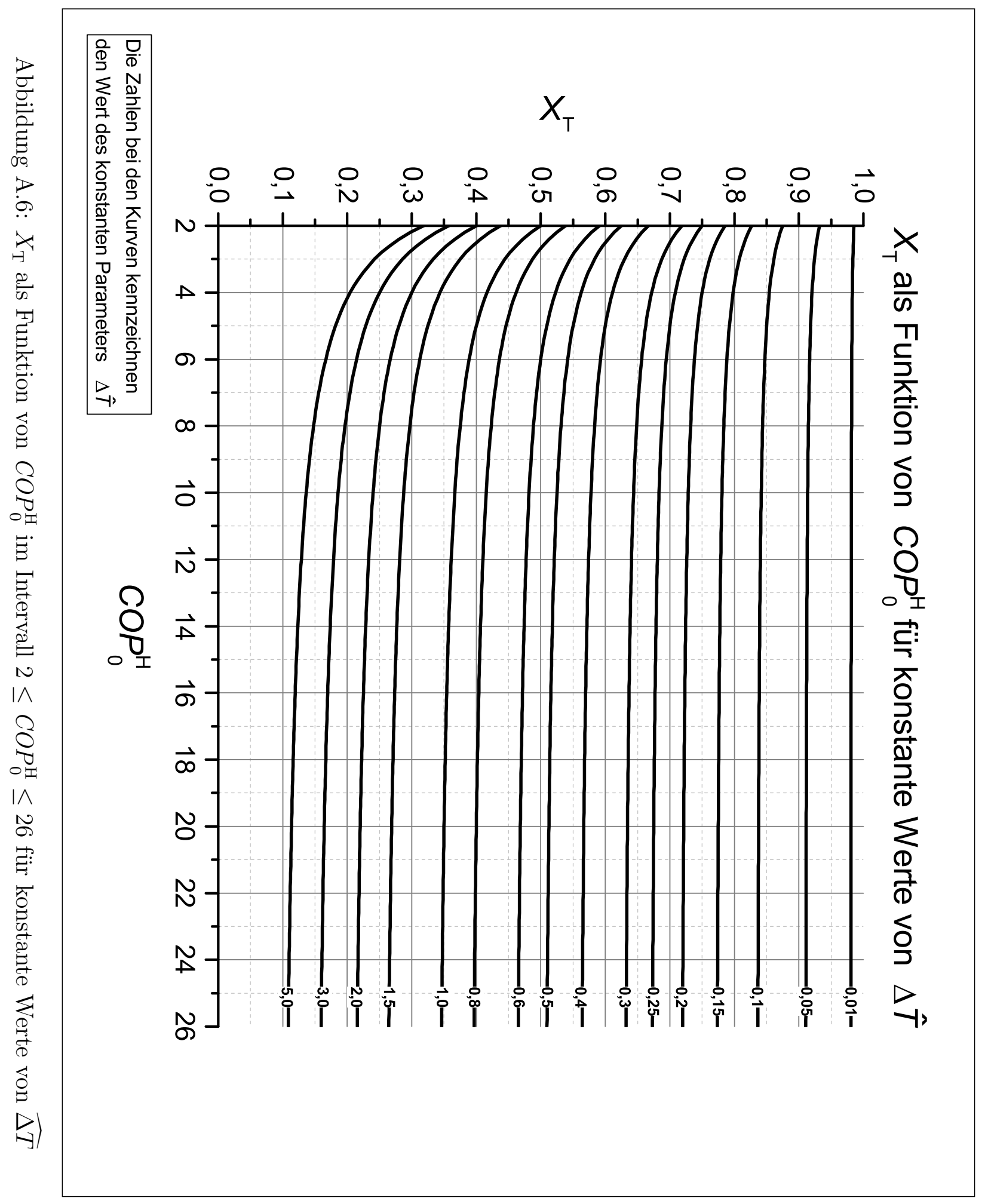




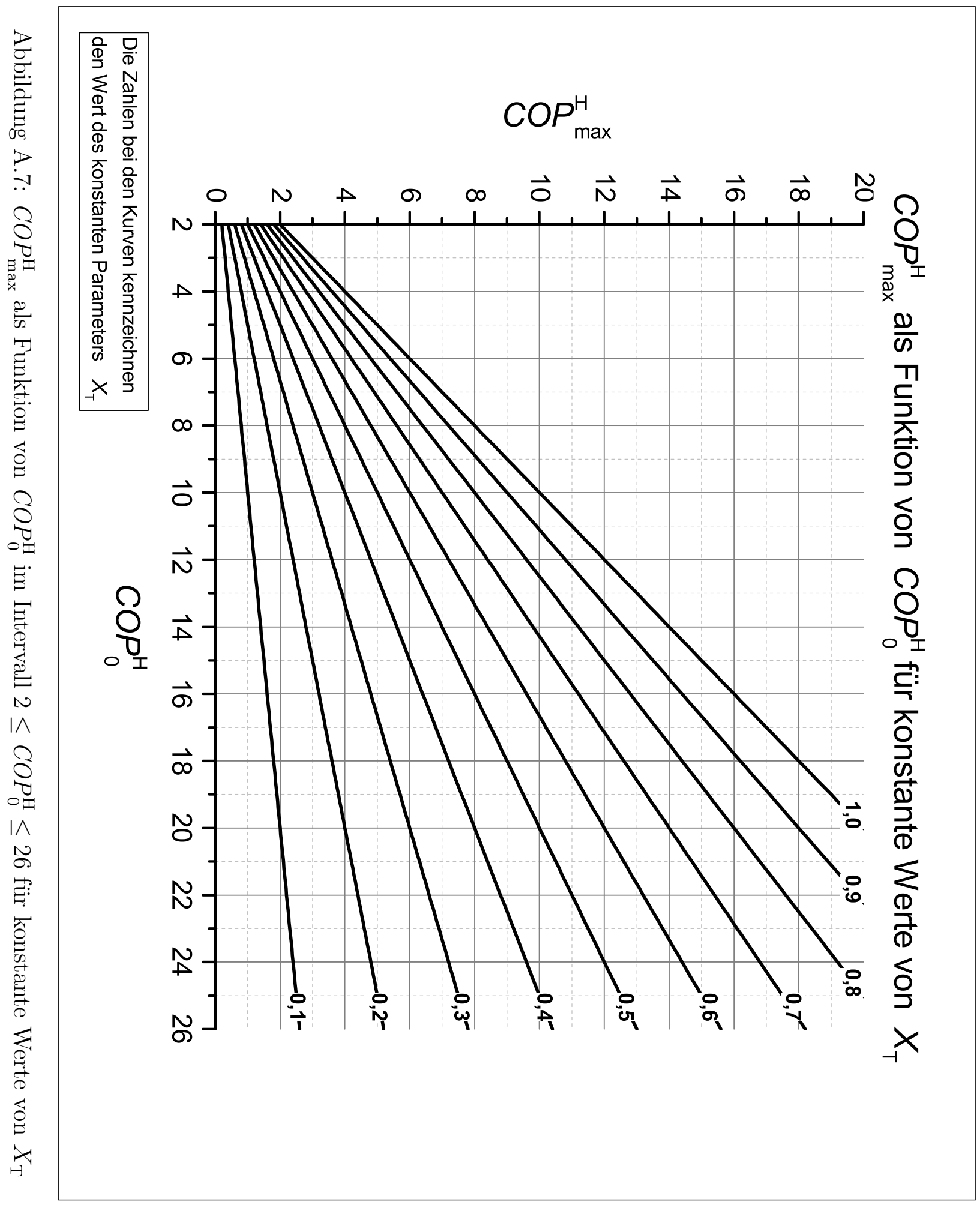




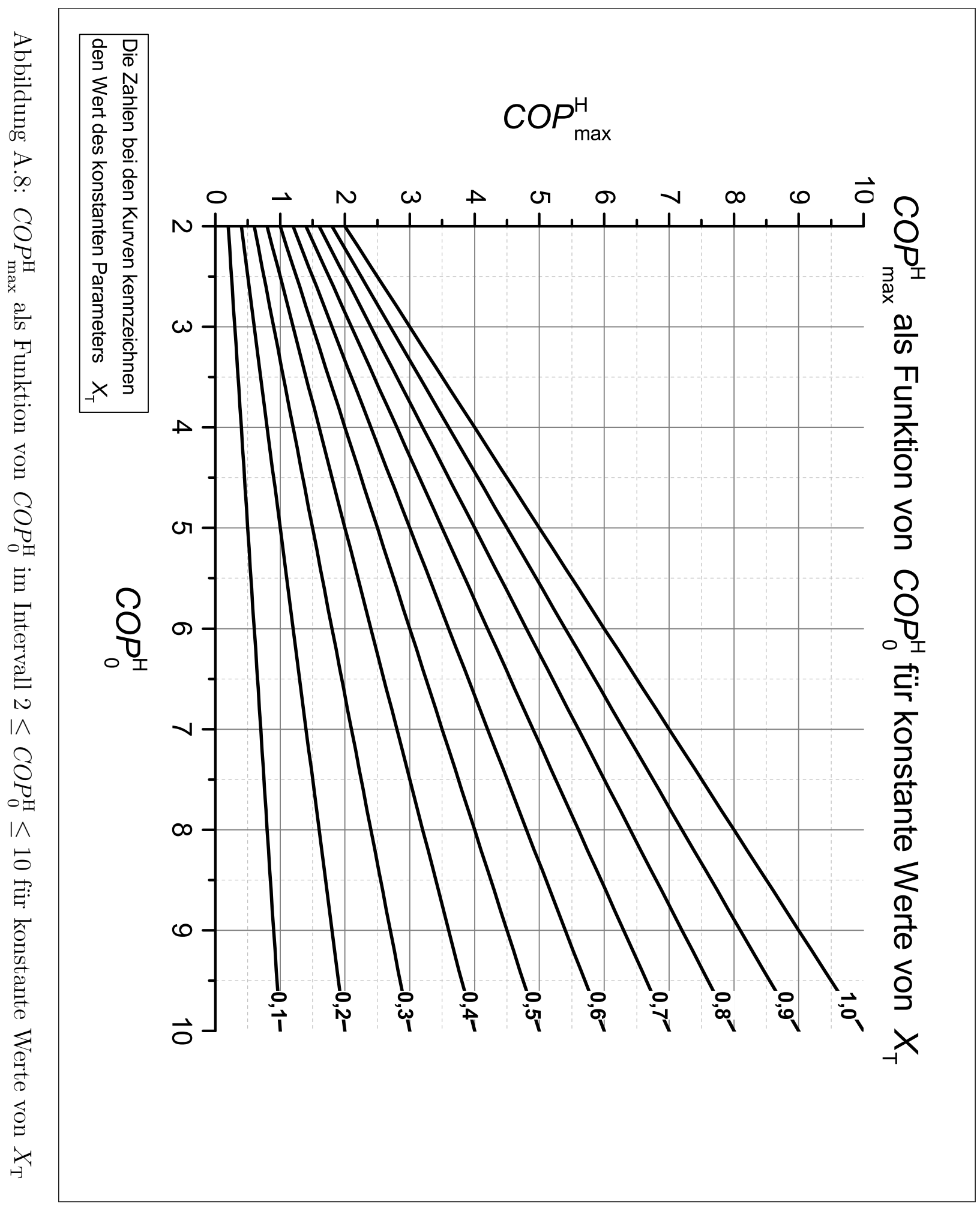




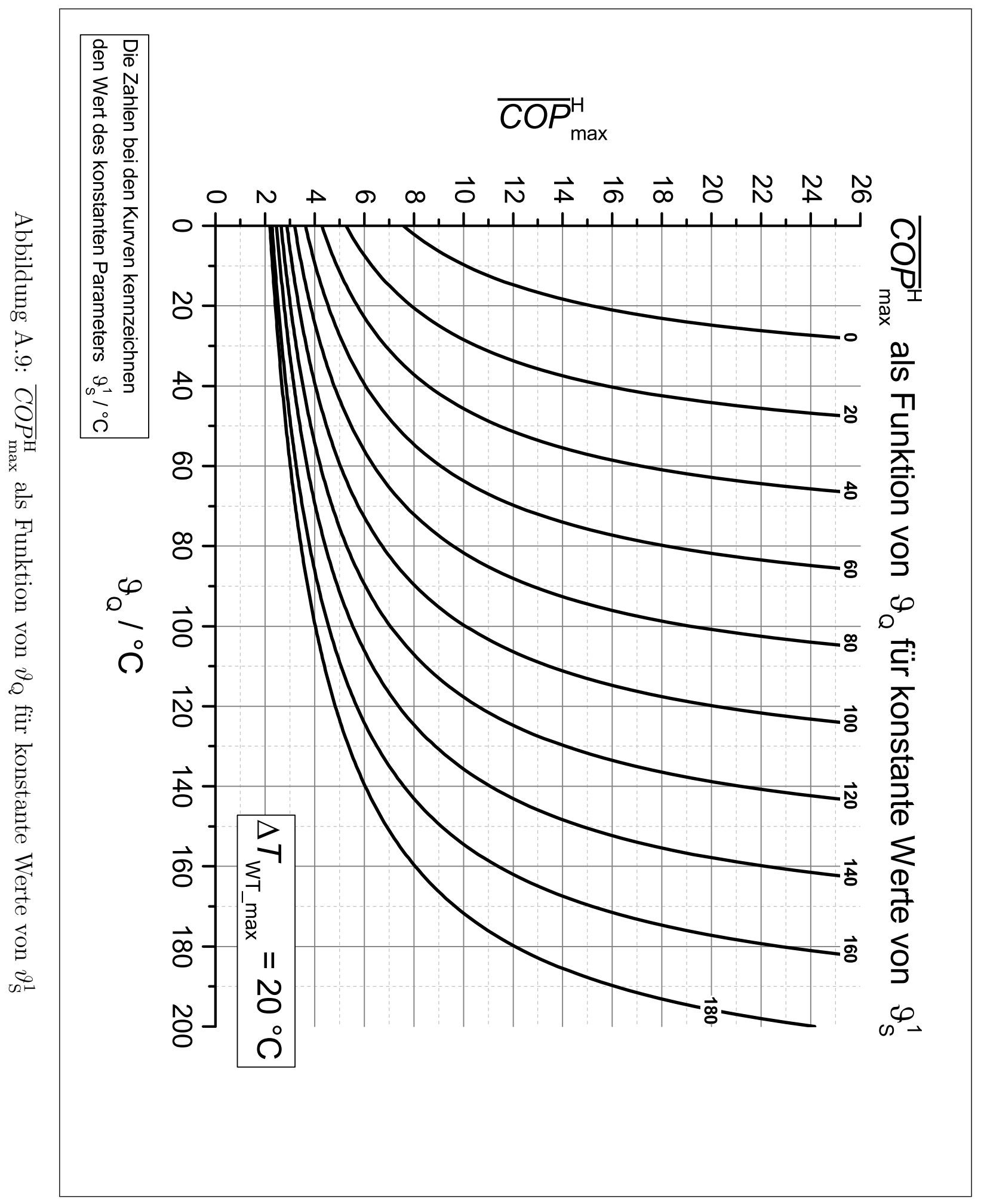




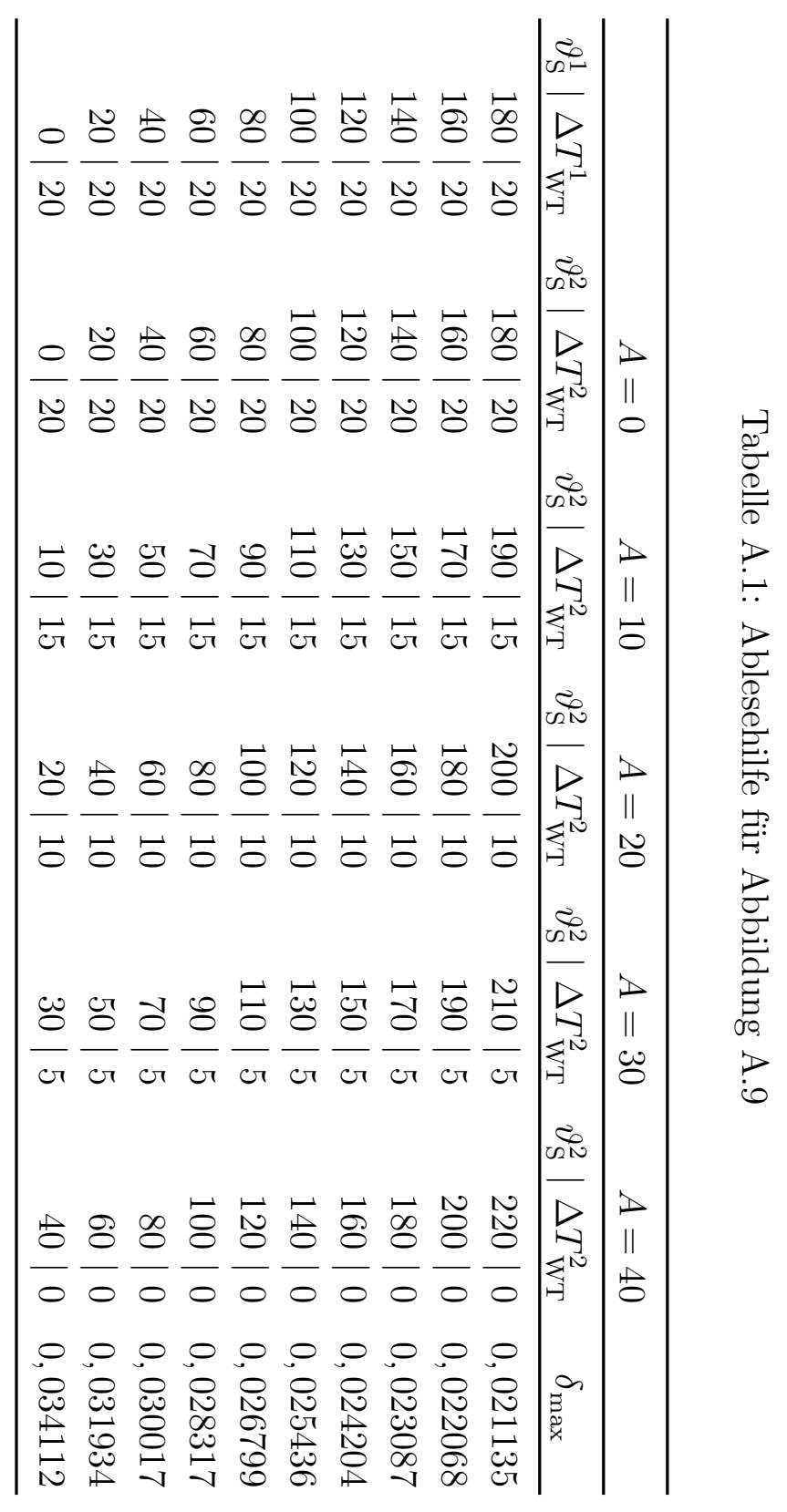




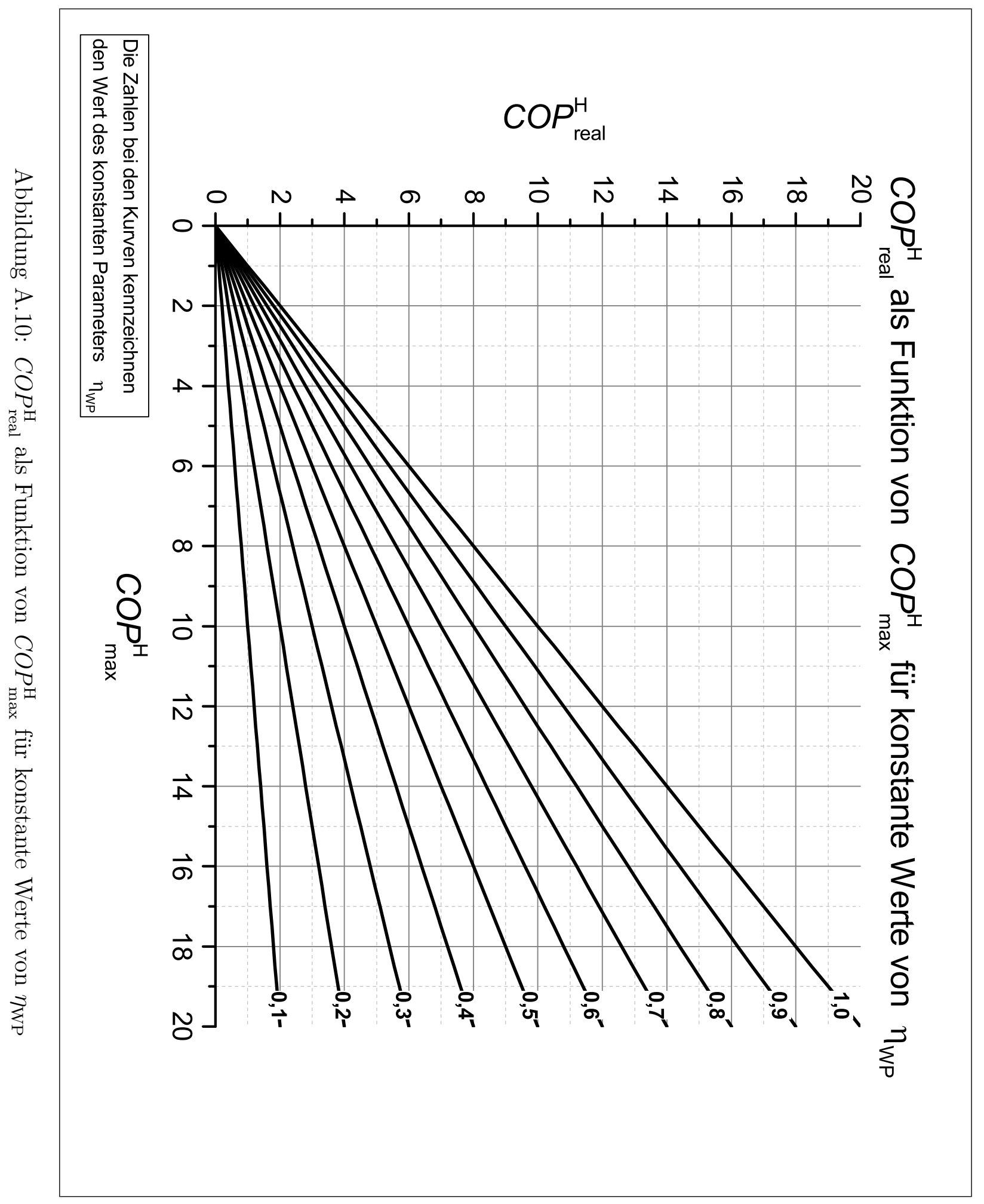




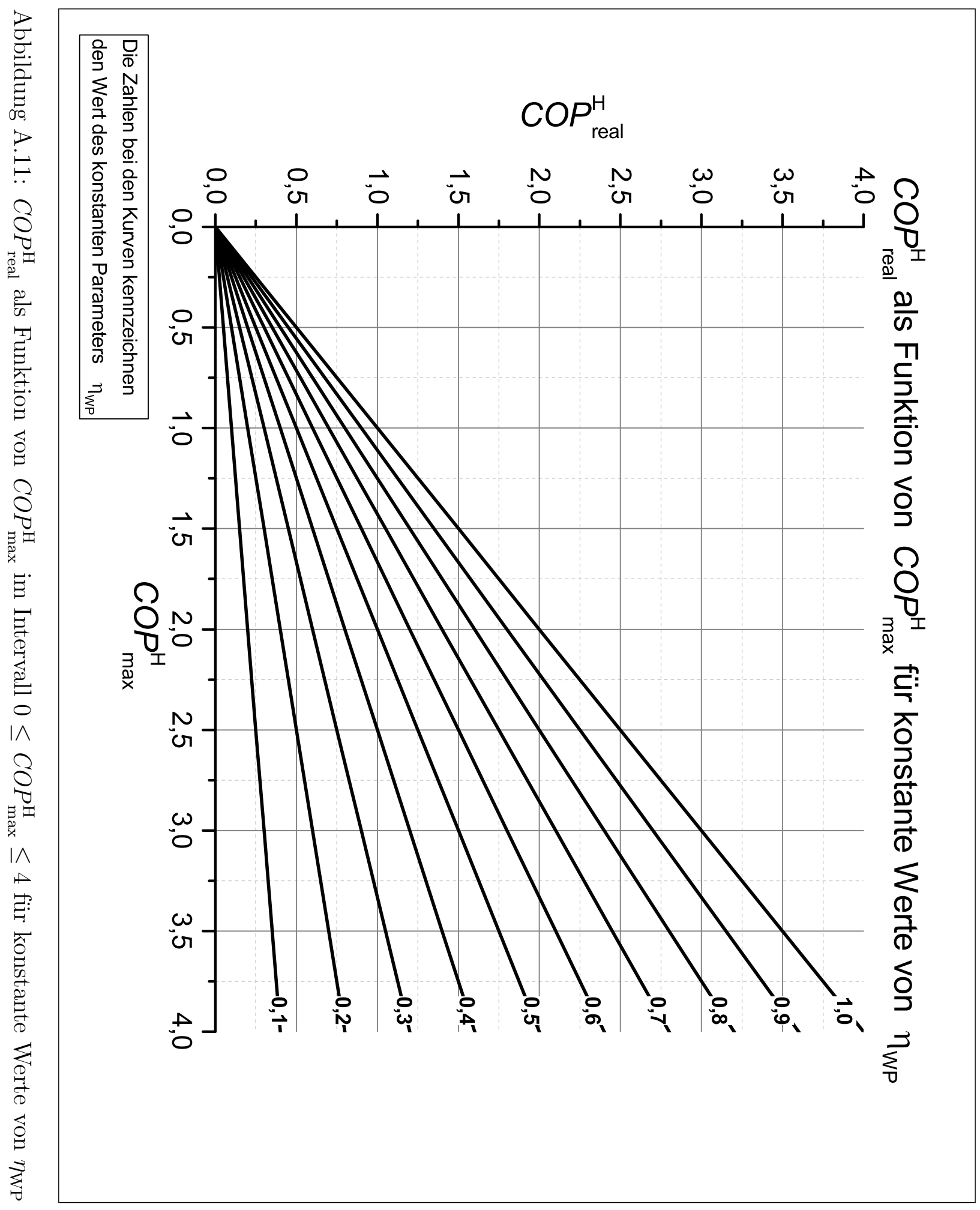




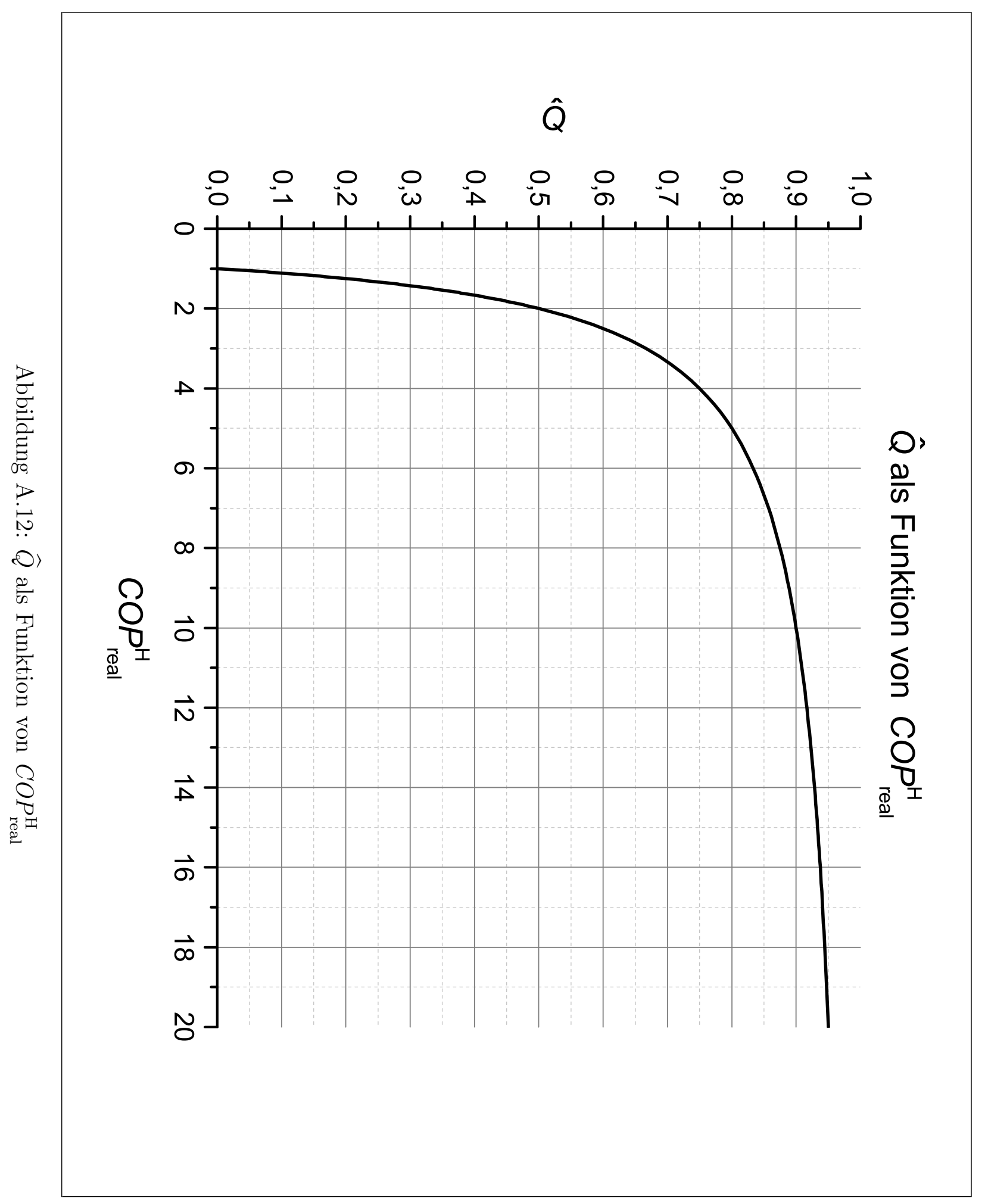




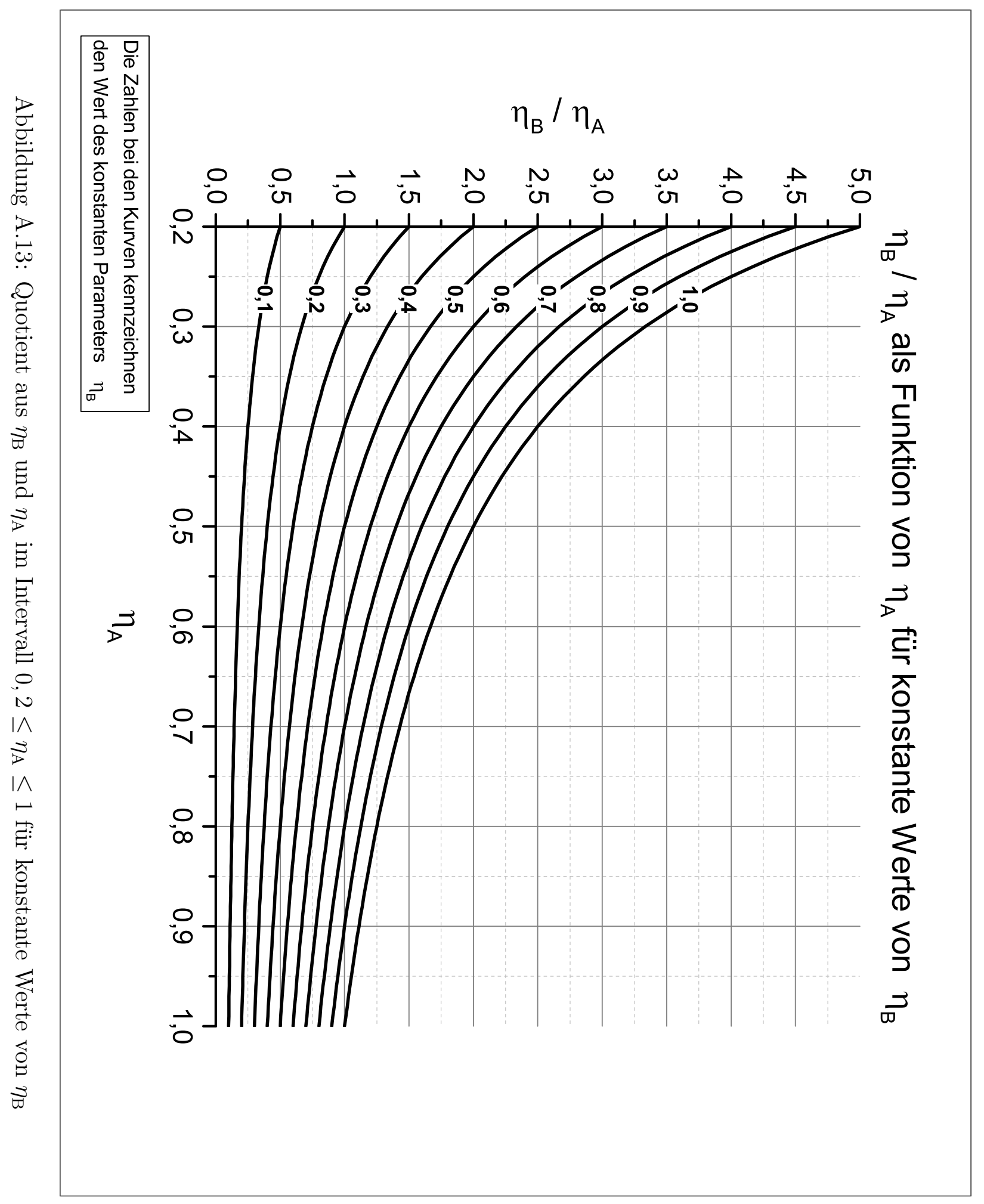




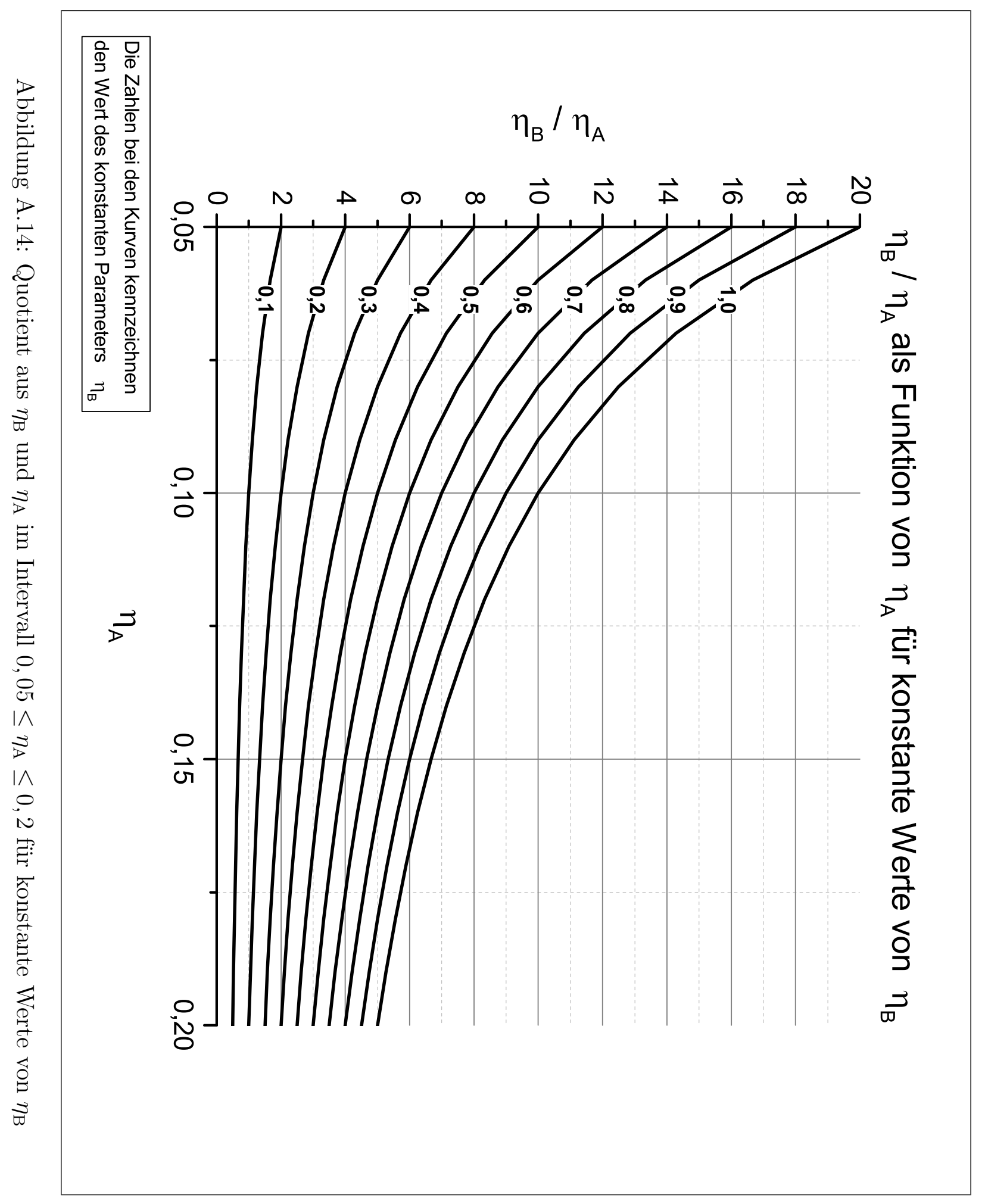




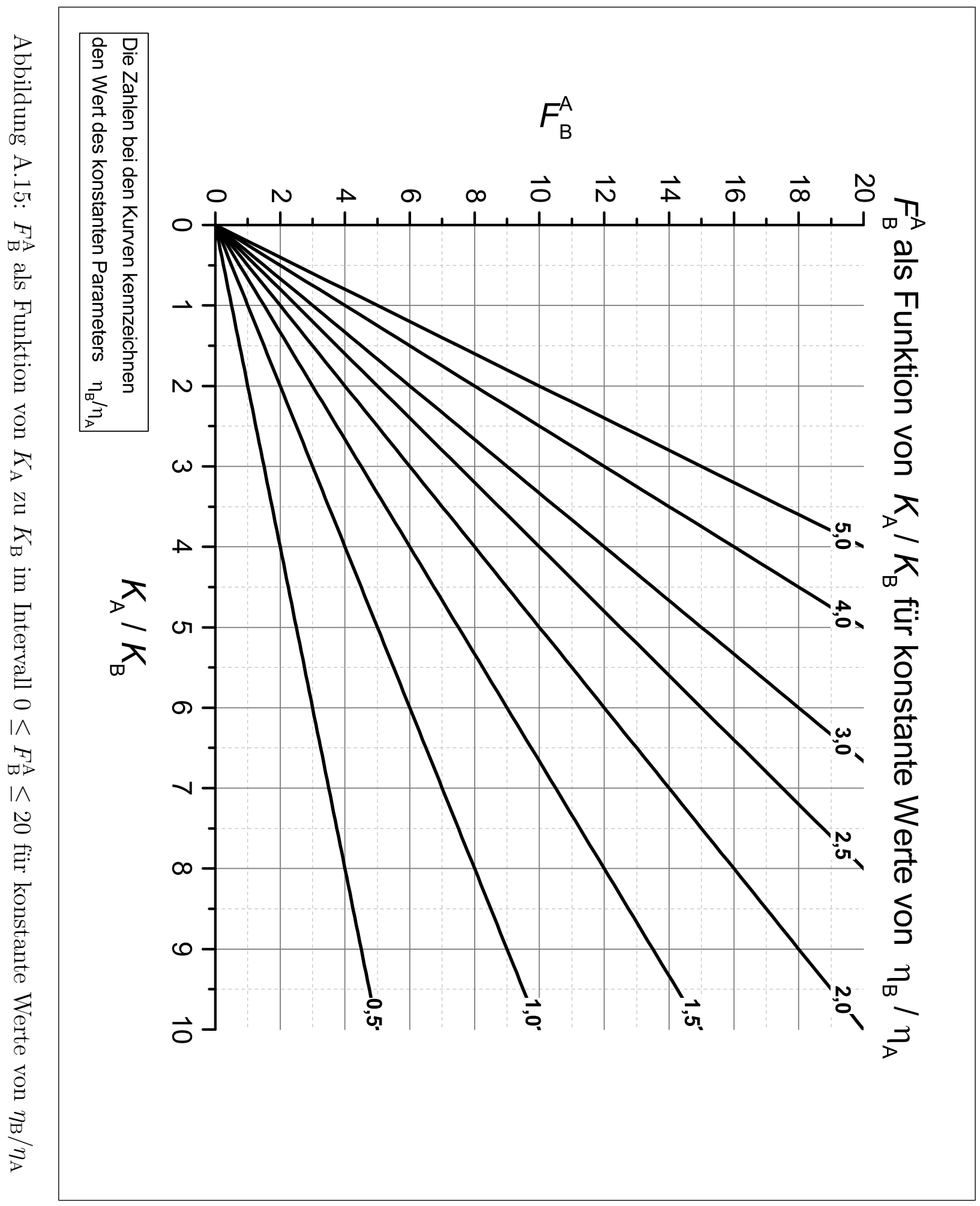




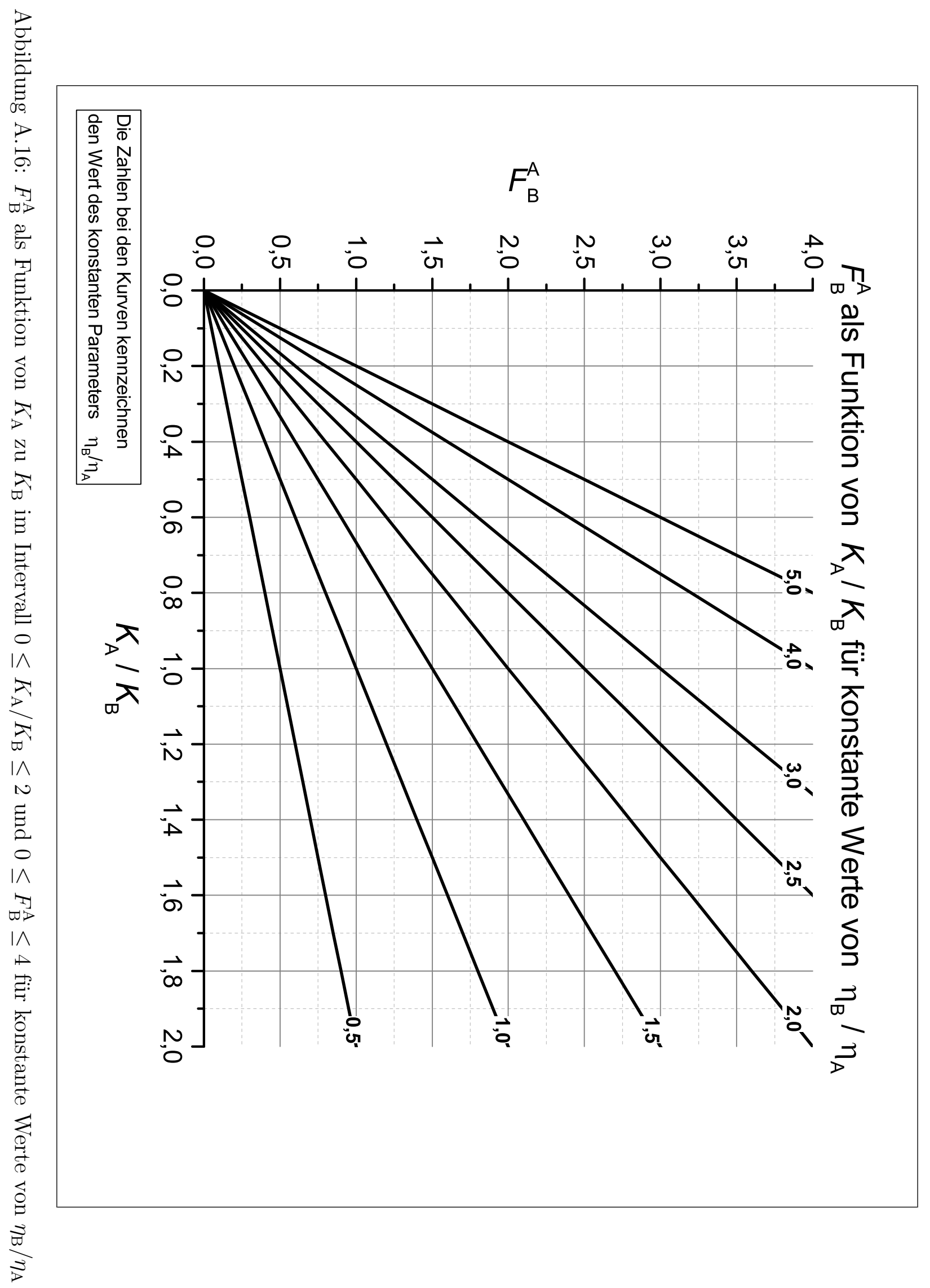




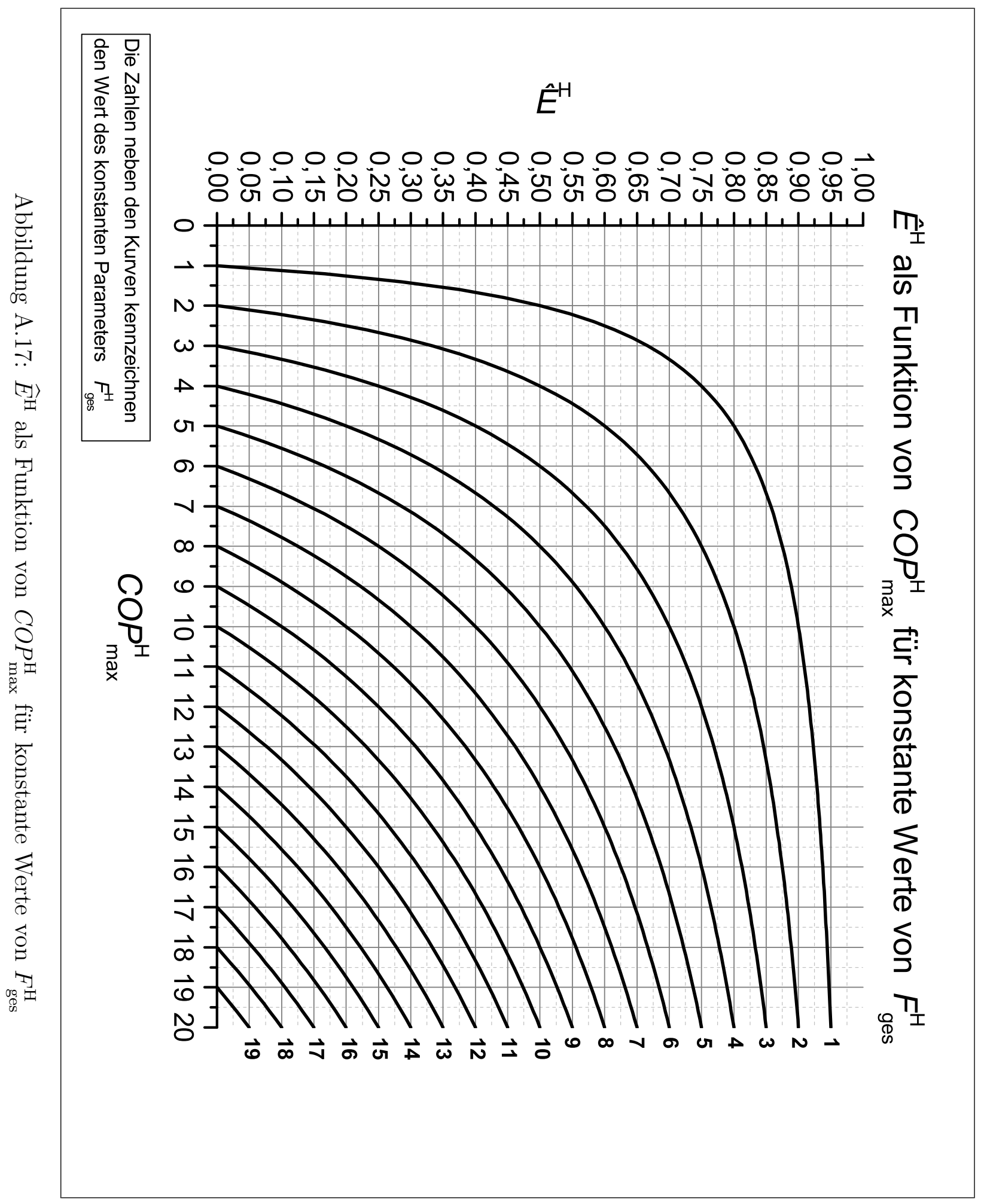




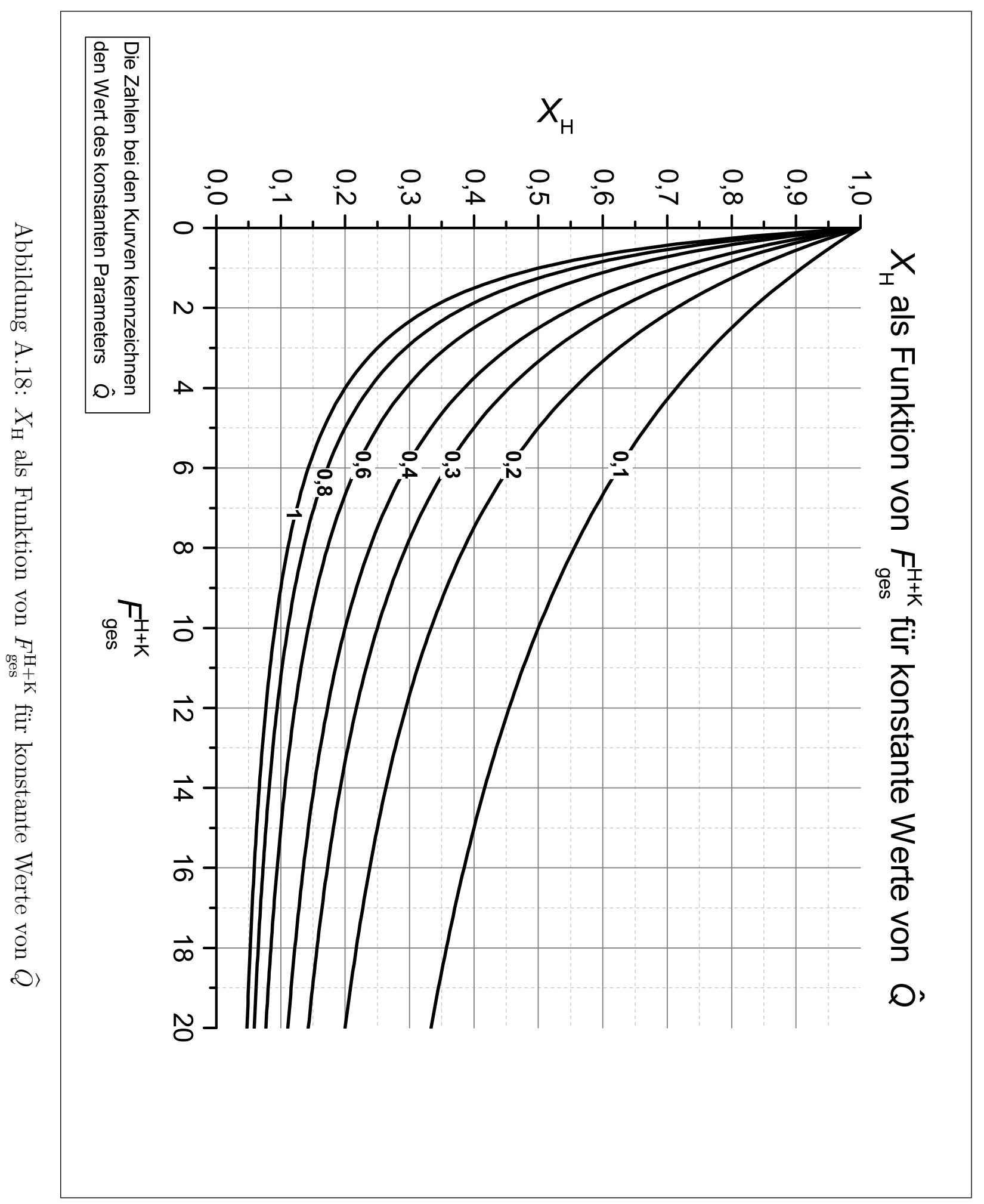




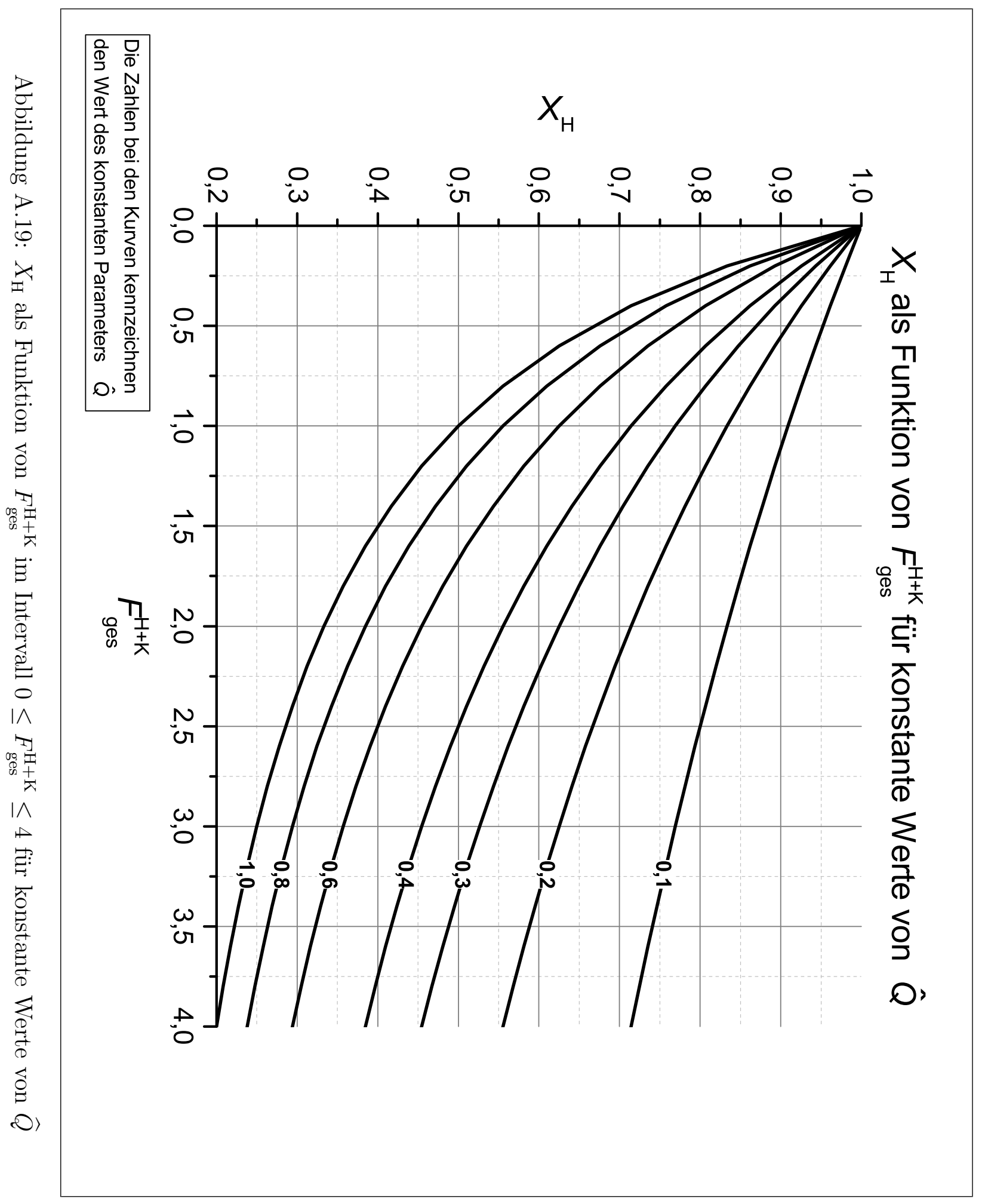




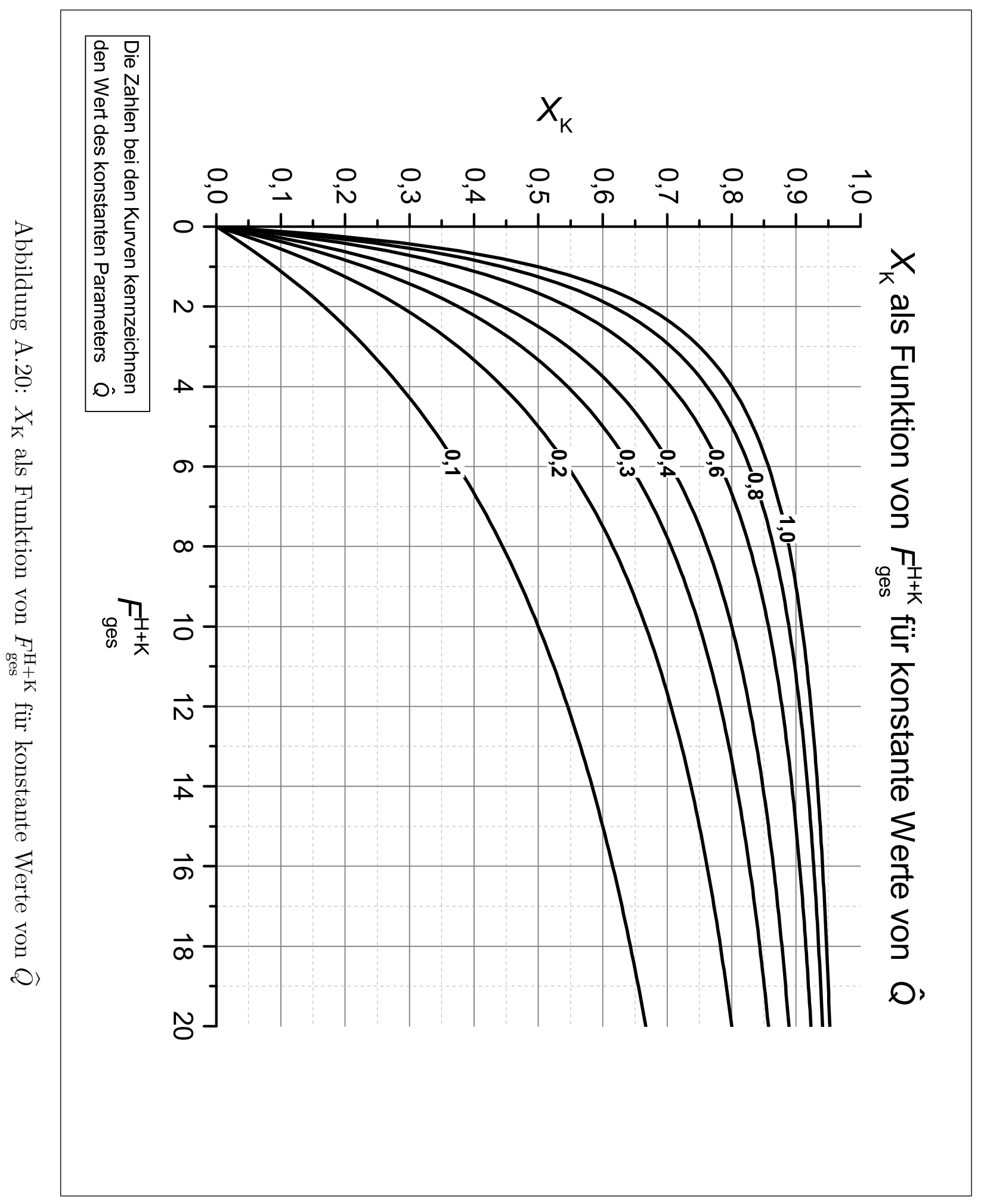




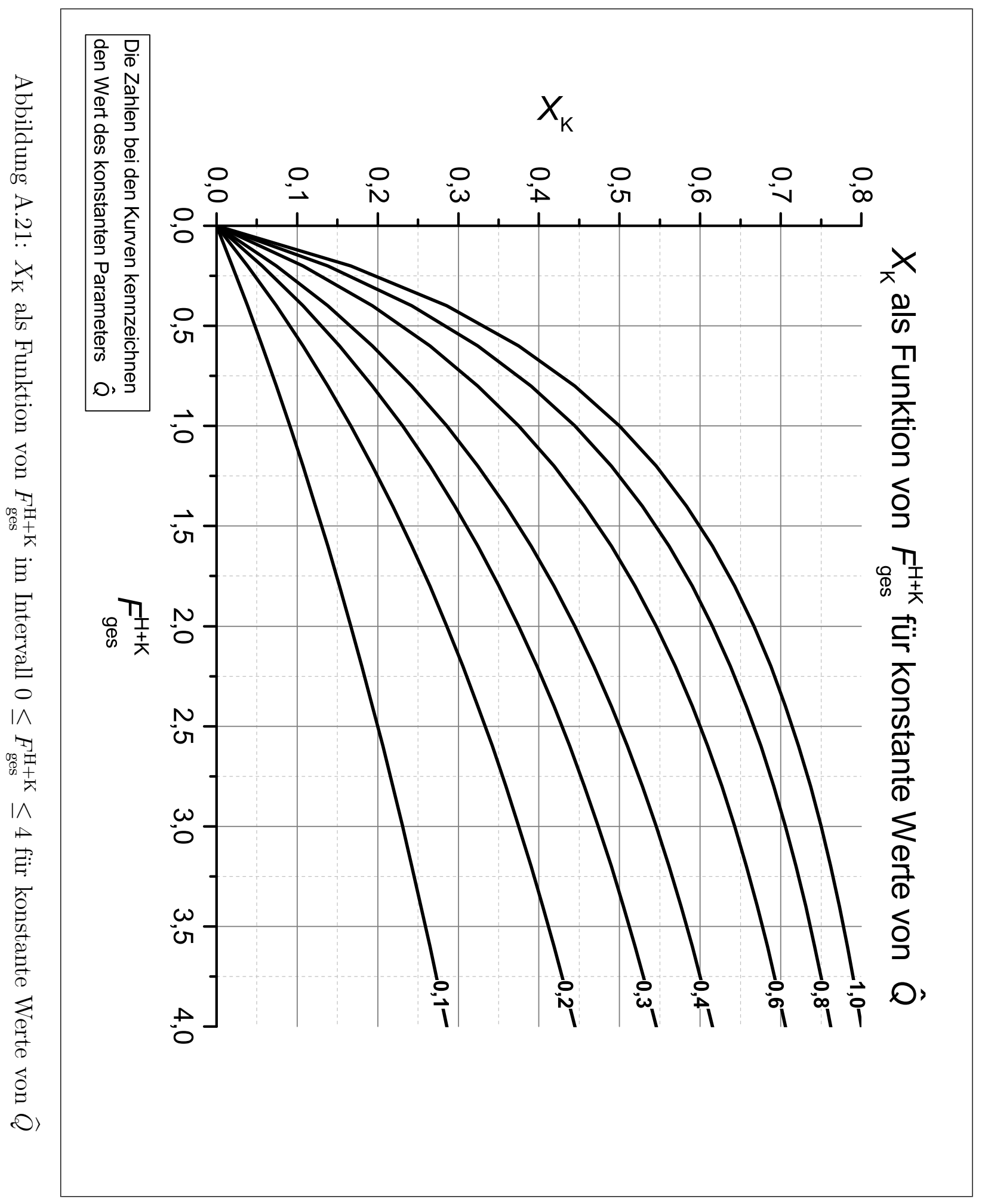




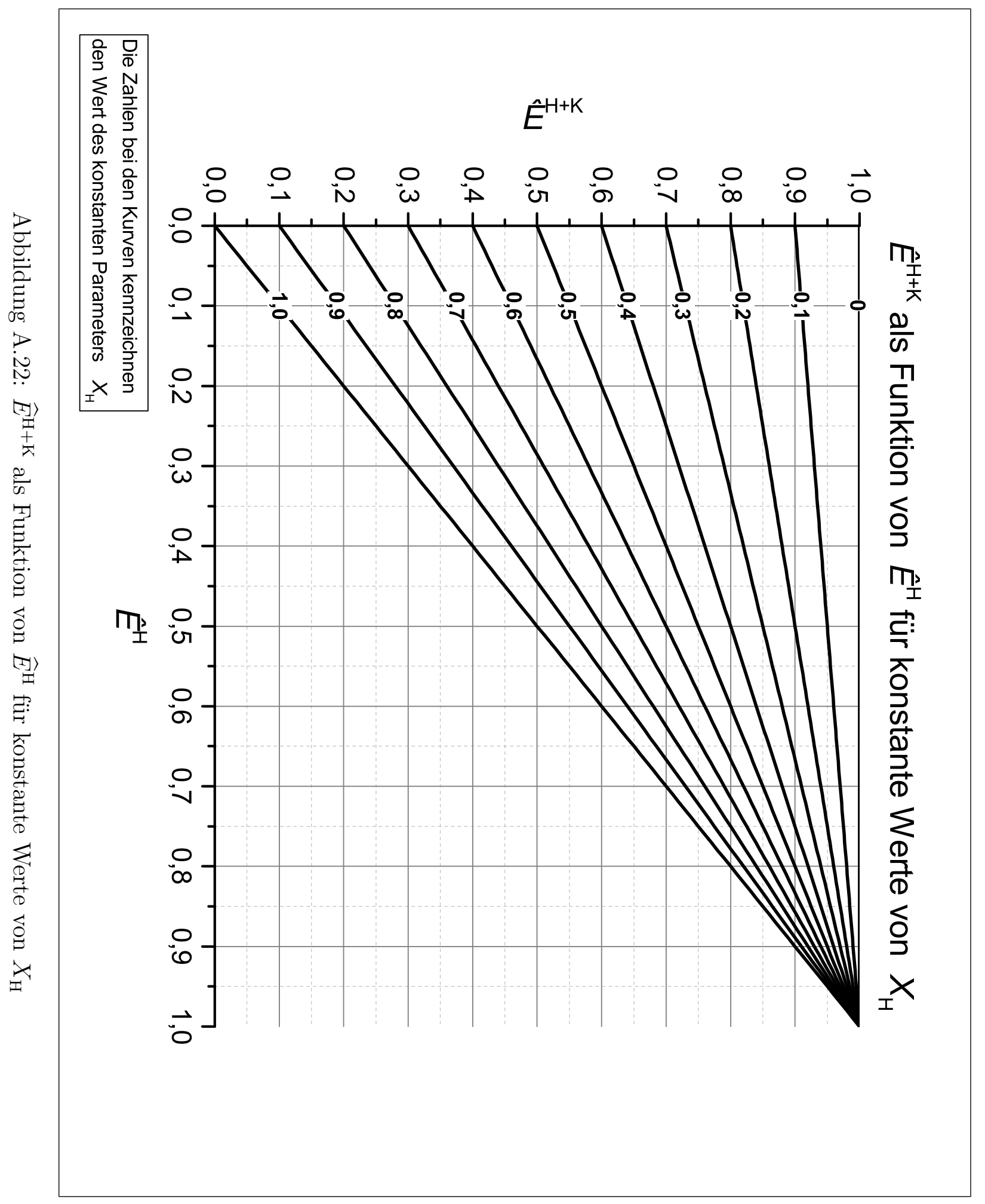




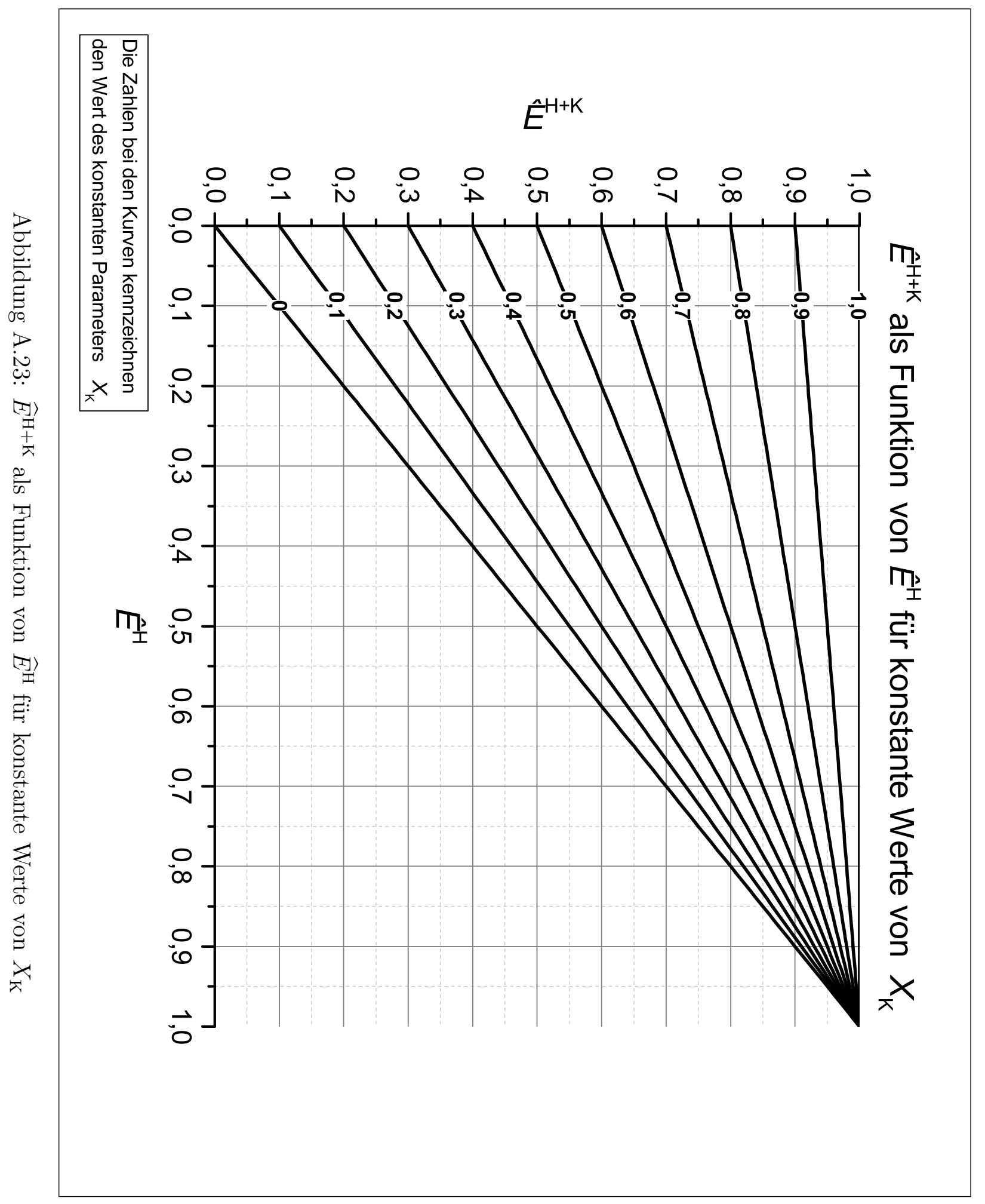

